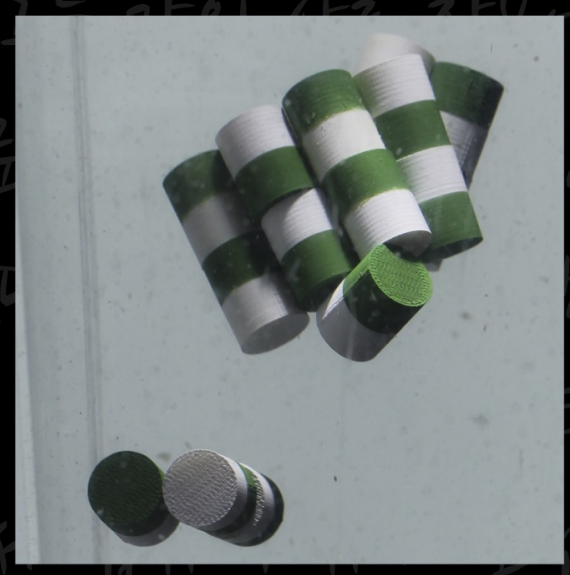

Observing magnetic objects in fluids

Tijmen A. G. Hageman

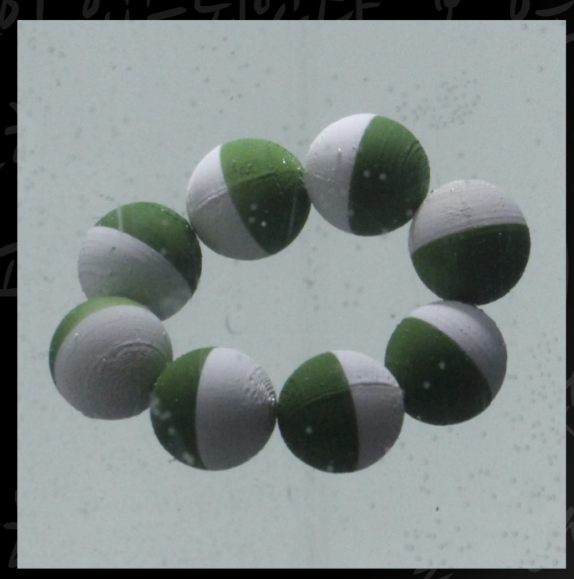

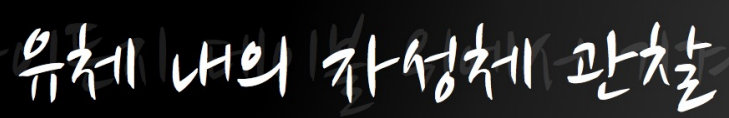





\title{
OBSERVING MAGNETIC OBJECTS IN FLUIDS
}

\author{
DISSERTATION
}

to obtain the degree of doctor at the University of Twente, on the authority of the rector magnificus, prof. dr. T.T.M. Palstra, and to obtain the degree of doctor at the Saarland University, on the autority of the president, prof. dr. M.J. Schmitt, on account of the decision of the graduation committee, to be publicly defended on Friday, 9 March 2018 at 16:45

\section{Tijmen Antoon Geert Hageman}

born on 25 May 1989,

in Didam, the Netherlands 
This dissertation is approved by

prof. dr. ir. L. Abelmann Saarland University (supervisor) prof. dr. ir. G.J.M. Krijnen University of Twente (supervisor) 


\section{Graduation committee}

prof. dr. J.N. Kok

University of Twente (chairman and secretary)

prof. dr. ir. L. Abelmann Saarland University (supervisor)

prof. dr. ir. G.J.M. Krijnen University of Twente (supervisor)

prof. dr.-Ing. M. Nienhaus Saarland University

prof. dr. T. Kraus

Saarland University

prof. dr. A. Manz

KIST Europe

prof. dr. M.C. Elwenspoek University of Twente

prof. dr. C.H. Slump University of Twente

\section{Deans}

prof. dr. J.N. Kok

University of Twente, faculty of Electrical Engineering, Mathematics and Computer Science

prof. dr. G. Kickelbick Saarland University, faculty of Natural Sciences and Technology

\section{UNIVERSITY OF TWENTE.}

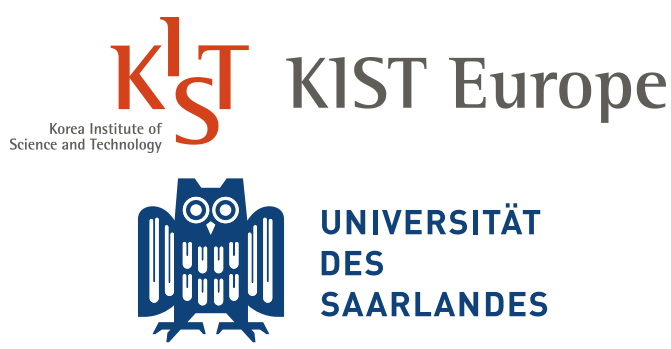

The research described in this dissertation was funded by KIST Europe.

Cover design by Tijmen Hageman and Caspar Abelmann

Printed by Ridderprint BV

(C) T.A.G. Hageman, Saarbrücken, Germany, 2018.

Electronic mail address: t.a.g.hageman@alumnus .utwente.nl

ISBN 978-90-365-4481-8

DOI $10.3990 / 1.9789036544818$ 



\section{Contents}

Contents

1 Introduction 1

1.1 The future of integrated circuits . . . . . . . . . . . . 1

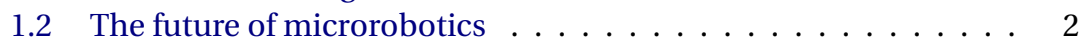

1.3 Research question . . . . . . . . . . . . . . . 4

1.4 Organisation of this thesis $\ldots \ldots \ldots \ldots$

2 Characterization of a macroscopic self-assembly reactor 7

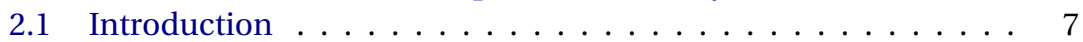

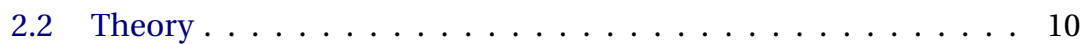

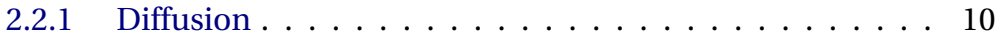

2.2 .2 Velocity distribution . . . . . . . . . . . . . . . . . . 12

2.2 .3 Drag coefficient . . . . . . . . . . . . . . 12

2.2 .4 Disturbing energy . . . . . . . . . . . . . . . 13

2.3 Materials and methods . . . . . . . . . . . . . . . . . 14

2.3.1 Reactor .................... 14

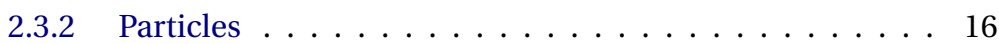

2.3.3 Reconstruction . . . . . . . . . . . . . . 17

2.4 Results . . . . . . . . . . . . . . . . . 17

2.4 .1 Single particles . . . . . . . . . . . . . 17

2.4 .2 Two-sphere results . . . . . . . . . . . . . 20

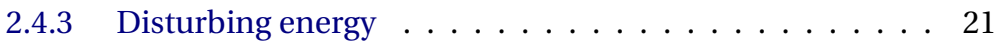

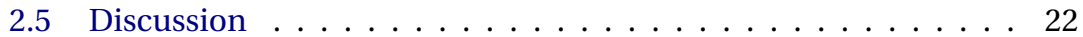

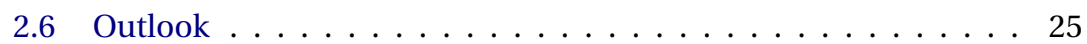

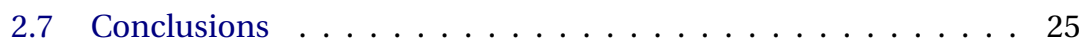

3 Control of disturbing energy in self-assembly 29

3.1 Introduction . . . . . . . . . . . . . . . . . 29

3.2 Theory ....................... 30

3.3 Methods..................... 31

3.3 .1 Flow calibration ................ 33

3.3 .2 Particles . . . . . . . . . . . . . . 34

3.3.3 Reconstruction . . . . . . . . . . . . . . . . 34 
3.3.4 Measurement precision . . . . . . . . . . . . 34

3.4 Results ....................... 35

3.4.1 Relation between flow asymmetry and disturbing energy 36

3.4.2 Directional dependency of disturbing energy . . . . . . 38

3.5 Discussion . . . . . . . . . . . . . . . 42

3.5.1 Directionality in turbulent flow . . . . . . . . . . . . . . 42

3.5.2 Richardson cascade . . . . . . . . . . . . . . 42

3.6 Conclusions . . . . . . . . . . . . . . . . . 43

4 Dipolar chain and ring formation $\mathbf{4 5}$

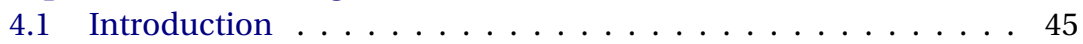

4.2 Theory...................... 46

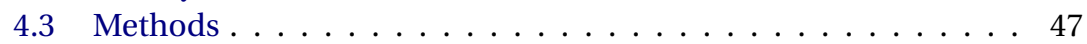

4.3 .1 Manual evaluation . . . . . . . . . . . . . . . . . 48

4.3.2 Automated evaluation . . . . . . . . . . . . . 48

4.4 Results . . . . . . . . . . . . . . . . . . . 48

4.4 Human evaluation . . . . . . . . . . . . . . . . . 49

4.4 .2 Automated evaluation . . . . . . . . . . . . . . 49

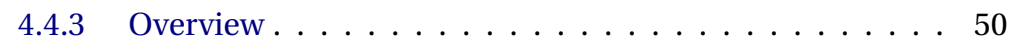

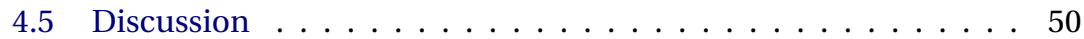

4.6 Conclusions ..................... 53

5 Three-dimensional cluster growth of magnetic particles 55

5.1 Introduction . . . . . . . . . . . . . . . 55

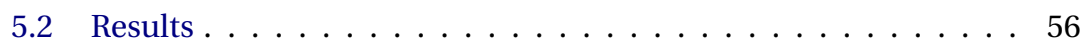

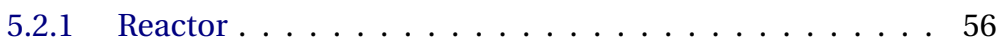

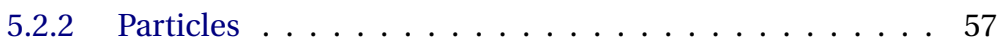

5.2 .3 Experiments . . . . . . . . . . . . 57

5.3 Conclusions .................... 58

6 Kinetics of 2D contrained orbitally-shaken particles 63

6.1 Introduction . . . . . . . . . . . . . . . . 63

6.2 Experimental setup . . . . . . . . . . . . . . . . 64

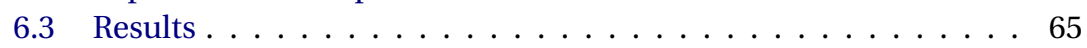

6.3 .1 Velocity distribution ............... 66

6.3 .2 Translational diffusion . . . . . . . . . . . . . . 69

6.3.3 Relative translational diffusion . . . . . . . . . . 70

6.3.4 Rotational diffusion ............... 71

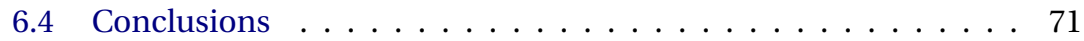

7 Magnetic response of MSR-1 75

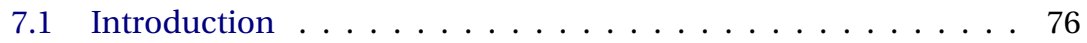

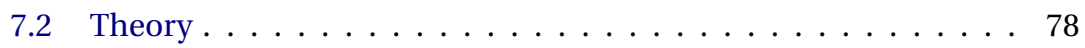

7.2.1 The Rate of Rotation . . . . . . . . . . . . . 78

7.2.2 U-turn trajectory simulations . . . . . . . . . . 85 
7.3 Experimental ........................ 87

7.3.1 Magnetotactic bacteria cultivation . . . . . . . . . 87

7.3.2 Dynamic viscosity of growth medium . . . . . . . . . 87

7.3 .3 Microfluidic Chips . . . . . . . . . . . . . . . . 89

7.3.4 Magnetic Manipulation Setup . . . . . . . . . . . . . 89

7.3.5 Macroscopic Drag Setup . . . . . . . . . . . . . . . 91

7.3 .6 Image Processing . . . . . . . . . . . . . . . 91

7.4 Results and discussions . . . . . . . . . . . . . . . 93

7.4.1 Estimate of model parameters . . . . . . . . . . 95

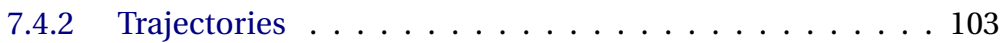

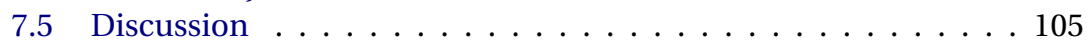

7.6 Conclusion . . . . . . . . . . . . . . 105

8 Real-time growth monitoring of MSR-1 107

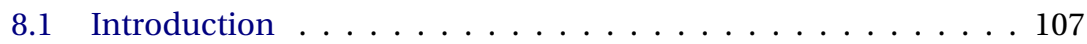

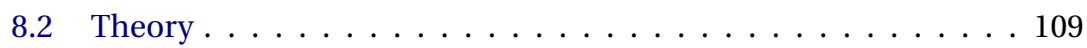

8.3 Materials and methods . . . . . . . . . . . . . . 110

8.3.1 Angle dependency of OD . . . . . . . . . . . 111

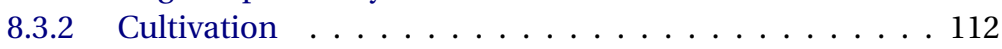

8.3.3 Growth experiment ................. 112

8.4 Results . . . . . . . . . . . . . . . . . . . 113

8.4.1 Angle dependency of OD . . . . . . . . . . . . . 113

8.4 .2 Growth curve ................... . . 113

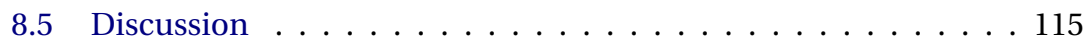

8.5.1 Repeatability and representability . . . . . . . . . . . 115

8.5.2 Response of MTB to magnetic fields . . . . . . . . . . 116

8.6 Conclusions .................... 116

9 Conclusion $\quad 119$

9.1 Macroscopic self-assembly . . . . . . . . . . . . . . . . . 120

9.2 Magnetotactic bacteria . . . . . . . . . . . . . . . 122

$\begin{array}{lr}\text { Appendices } & 123\end{array}$

A Velocity distribution of particles in the orbital shaker 125

B Growth curves measured in the OD meter 127

$\begin{array}{lr}\text { Bibliography } & 132\end{array}$

$\begin{array}{lr}\text { Abstract } & 144\end{array}$

$\begin{array}{lr}\text { Samenvatting } & 146\end{array}$

$\begin{array}{ll}\text { Zusammenfassung } & 148\end{array}$ 
초록

Acknowledgments

152

Publications

154

Biography

158 


\section{Chapter 1}

\section{Introduction}

In the modern day world we can observe a trend towards miniaturization of technology. And this is not without reason, as this can lead to for instance more functionality in the same device and reduce the amount of resources used for their construction or consumed during operation.

\subsection{The future of integrated circuits}

A well-known example is integrated circuits, pioneered by Jack Kilby in 1958, which have been subject to Moore's scaling law. These predominantly twodimensional architectures get more functionality over time by reducing the transistor dimensions so that more fit on the same chip, a process limited by the resolution of lithography processes. The industry has recently reached the $10 \mathrm{~nm}$ node for lithography, but cannot scale indefinitely due to the limits imposed by the size of atoms. To continue the trend of progress, besides changing the computational approach (such as quantum computing), we will eventually have to move to the third dimension.

This is in need of new production methods as lithography-based methods like layer stacking and interference lithography are severely restricted in the third dimension. Self-assembly of colloidal particles with embedded electronics into regular 3D structures would be a solution (figure 1.1) (Abelmann et al., 2010). The formation of colloidal crystals has been studied by the likes of Alfons van Blaaderen (1997; 2013) and Albert Philipse (Philipse et al., 1994; Rossi et al., 2011), building on fundamental concepts of (molecular or macroscopic) selfassembly pioneered by George Whitesides (Grünwald et al., 2016; Whitesides and Grzybowski, 2002). The dynamics of microscopic self-assembly are difficult to observe due to the small size and short characteristic time constants involved, and therefore most publications are focused on reaction products. Yet, it is important to fully understand the particle-particle and particle-fluid interaction to optimize the assembly process and to minimize crystal defects. Computer simulations are a solution, but these scale unfavourable with the amount of particles 

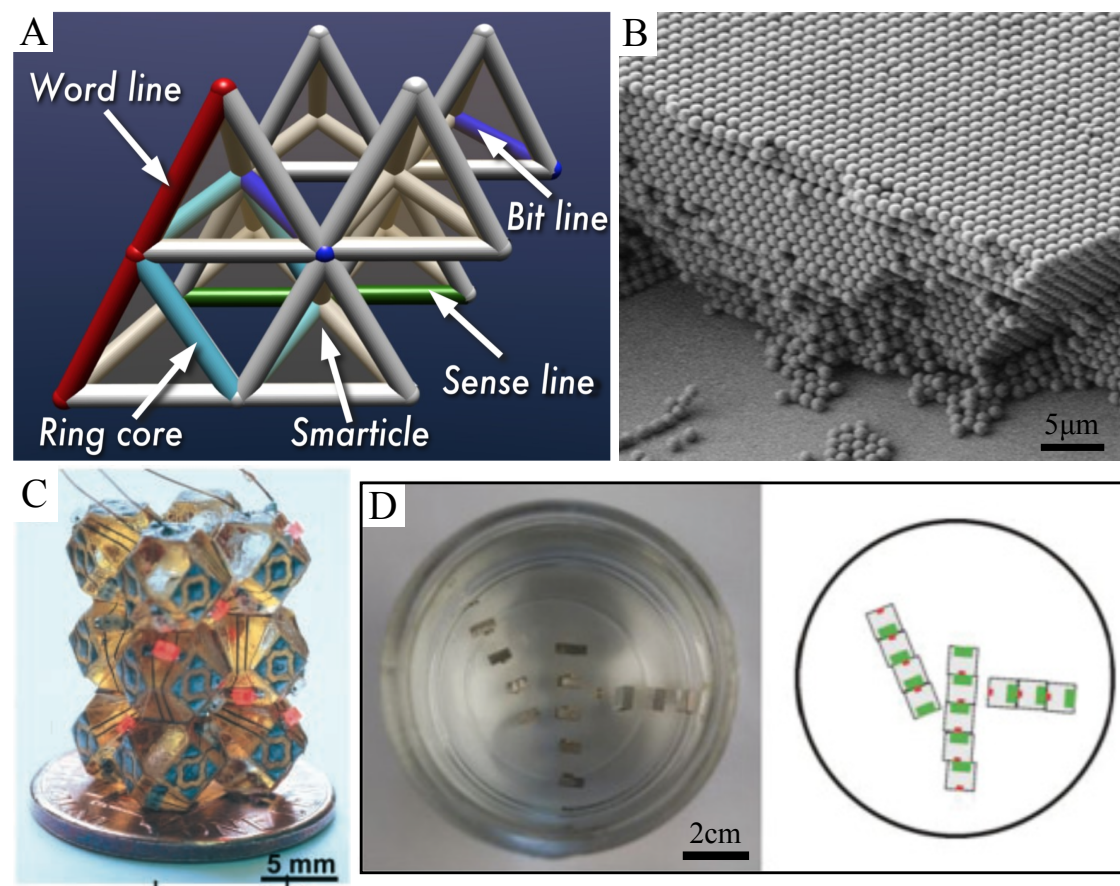

FIGURE 1.1 - Three-dimensional electronics like memory chips could be constructed from smart building blocks, "smarticles" (A) (Abelmann et al., 2010), by forming regular structures via colloidal self-assembly (B) (Norris et al., 2004). Smart particles have been successfully self-assembled on macroscopic scale, forming electrically functional networks (C) (Gracias et al., 2000). Self-assembly dynamics may be simulated on macroscopic scale (D) (Ilievski et al., 2011b).

involved. Analogue simulations in the form of a macroscopic self-assembly reactor can act as an alternative, greatly increasing visibility. For a proper analogy we need an interplay between the disturbing (temperature) and assembling (electrostatic, magnetic, van der Waals etc.) forces present on microscopic scale. Such forces could be respectively turbulence and magnetic dipole interaction, as explored before by Filip Ilievski (2011b).

\subsection{The future of microrobotics}

A second example of miniaturization is the use of micro-robotics. Among the many applications of miniature robots are their use to further reduce the invasiveness of surgical procedures. By injecting them in the blood stream they can carry out targeted drug delivery or more complex procedures from within the body. With current state-of-art technology it is still very difficult to produce 


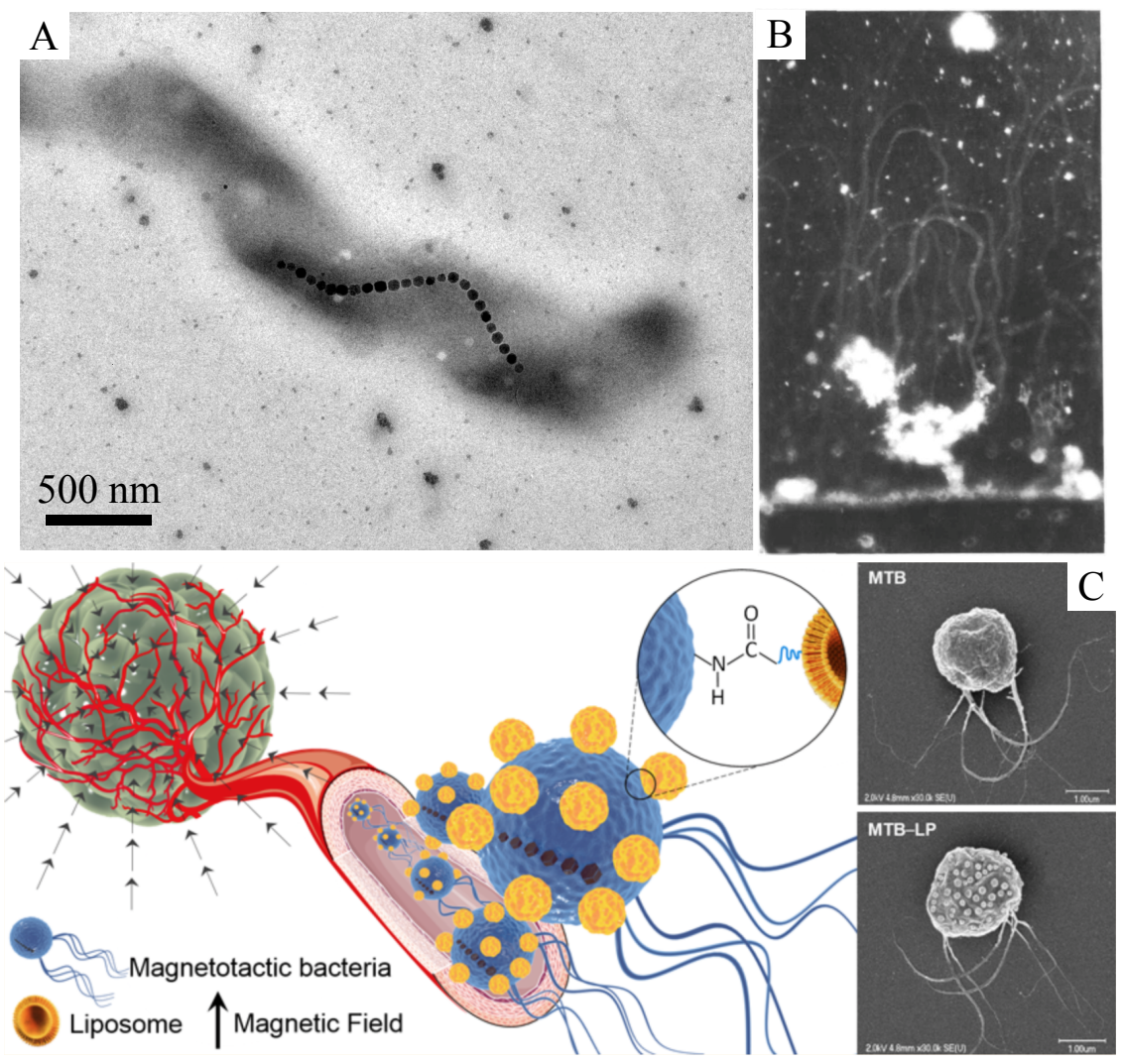

FIGURE 1.2 - Magnetotactic bacteria (MTB) (A) grow magnetic crystals to align with magnetic fields. When exposed to a sudden field inversion, these bacteria make U-shaped trajectories (B) (Esquivel and Lins de Barros, 1986). MTB might be used as directed drug delivery agents for tumours (C) (Taherkhani et al., 2014).

robots small and functional enough to accomplish these tasks. Nature, however, supplies us with self-propelled, self-replicating, magnetically steerable robots that can harvest their energy from the environment: magnetotactic bacteria (MTB). These bacteria develop chains of magnetic nano-crystals to align with the earth magnetic field, to convert a random three-dimensional search into a more efficient one-dimensional search towards their ideal habitat. Sylvain Martel $(2016$; 2009) has pioneered the use of MTB as drug delivery agents to tumours (figure 1.2).

We need to have a good understanding of the exact properties such as the swimming behaviour of these organisms and their response to magnetic fields 
to make their motion predictable and thereby making them useful robots. Such studies have been carried out by Darci Esquivel (1986) and Kaspars Ērglis (2007). However, due to the fact that these bacteria are difficult to cultivate and visualize, systematic characterisation and statistics of the motion of MTB have not been obtained. Although sophisticated time-lapse imaging and tracking have been available since the development of digital camera's, reliable confinement of micro-organisms to very shallow planes is not yet common practice, with accompanying depth-of-field problems. Recent developments by the lab-on-achip community include combining live cells with microfluidics, allowing for such confinement $(<10 \mu \mathrm{m})$ which improves visibility and enables long-term tracking of individual micro-organisms.

\subsection{Research question}

This brings us to the central topic of this thesis. The two examples introduced deal both with magnetic particle-fluid systems of which we want to model their properties and dynamics. They represent vastly different domains, dealing with objects which are microscopic, alive and self-propelled versus macroscopic, dead and turbulence-driven. However, in both cases we aim to learn from their properties and dynamics via a method of observation, and try to answer the following question:

\section{WHAT CAN WE LEARN FROM OBSERVING MAGNETIC OBJECTS IN FLUIDS?}

The matured technology of digital camera's and wide application potential of image processing techniques allow for careful observation of the exact kinetics of individual objects. Comparing the information obtained from object trajectories allow us to estimate model parameters with statistical significance.

\subsection{Organisation of this thesis}

This thesis is organised in two parts, both focused on another topic. Chapter 2 introduces a macroscopic, turbulence driven self-assembly reactor and assesses how close the macroscopic environment resembles the microscopic realm. Chapter 3 describes how the disturbing energy of the reactor can be tuned and its affect on the behaviour of particles. These chapters only quantize the behaviour of single and two-particle systems. Chapter 4 extends this to four particles, and uses a machine learning approach to classify the state of such systems. Chapter 5 addresses the influence of aspect ratio, geometry and amount of particles on the structures formed by multi-particle systems. Chapter 6 takes a step back to the two-dimensional realm. Rather than turbulence we use friction caused by an orbital shaker to shake the particles, to see whether the source of disturbing energy is of importance for particle kinetics. 
Chapter 7 is the first chapter about MTB. It deals with estimating the parameters of individual MTB by observing their trajectories as a response to changes in the magnetic field. Chapter 8 introduces a machine which aims to measure parameters of an entire MTB population over time, based on solely optical density measurements. 


\title{
Chapter 2
}

\section{Characterization of a macroscopic self-assembly reactor}

\begin{abstract}
We have built and characterised a macroscopic self-assembly reactor which agitates magnetic, centimetre-sized particles by a turbulent water flow. By scaling up self-assembly processes to centimetre-sized objects also characteristic time constants scale drastically. This makes the system a physical simulator of microscopic self-assembly, where the interaction of inserted particles are easily observable. Trajectory analysis of single particles reveal their velocity to be Maxwell-Boltzmann distributed and show that their average squared displacement over time can be modelled by a confined random walk model, demonstrating a high level of similarity to Brownian motion. The interaction of two particles has been modelled and verified experimentally by observing the distance between two particles over time. The disturbing energy (analogue to temperature) obtained experimentally is increasing with sphere size, and differs by an order of magnitude between single- and two sphere systems (approximately $80 \mu \mathrm{J}$ versus $6.5 \mu \mathrm{J}$ respectively).

The work in this chapter is a team effort. My contribution includes composing the theoretical background, building and programming and calibrating the dual-camera system and performing the data analysis. I wrote the software for particle detection, 3D-reconstruction, particle tracking and trajectory analysis and performed the statistical analysis. The reactor was constructed in Twente by Remco Sanders and Léon Woldering and was modified by Per Löthman, who also designed the particles and did the experiments.
\end{abstract}

\subsection{Introduction}

Self-assembly is the process in which a disorganised system assembles in a specific product without external interference. The final properties of the as- 
sembly are determined by the properties of the individual parts. Self-assembly is used extensively by nature; for example, in crystal growth, protein folding, the assembly of molecules into larger compounds, and the creation of complex organs such as the human brain.

Self-assembly is a prospective candidate for use in areas where conventional production and assembly methods are problematic. Although it is not limited to specific dimensions (Whitesides and Grzybowski, 2002), self-assembly is especially applicable to small scales (Elwenspoek et al., 2010) as, for example, conventional machining tools for three-dimensional construction are limited to larger feature sizes, while photo-lithography processes are two-dimensional in nature. The review of Mastrangeli et al. (2009) gives an excellent summary of selfassembly on small scales, ranging from nanosized DNA origami (Rothemund, 2006) to magnetically folded milli-scale structures (Iwase and Shimoyama, 2005).

Arguably one of the most promising applications will arise in the semiconductor industry. As a result of the continuous downscaling of fabrication processes, non-volatile data storage systems will at some point run into its limit to store and process bits of information using only a few atoms (Bennewitz et al., 2002). To achieve higher data densities, it is necessary to move to the third dimension. The first steps in this direction have been taken by stacking wafers (Dellutri et al., 2006) or layers (Tanaka et al., 2007). However, the stacking approach is not suitable to achieve truly three-dimensional structures, in which both the resolution and extent of the features is identical in all directions (Abelmann et al., 2010). We believe that the most promising production method is three-dimensional self-assembly.

Not only is three-dimensional self-assembly a prospective candidate for highly repetitive memory structures, it will also open a path for more complex electronics, such as processors. For instance, Gracias et al. (2000) have designed millimetre-sized polyhedra with integrated electronics. By self-assembling these into crystals, functional electrical circuits have been demonstrated on a centimetre-scale. Scaling down the building blocks is a crucial step towards scalability of the system as a whole.

It has been demonstrated that microscopic spherical particles can form regular structures up to centimeter-sized dimensions (Philipse, 1989). By tuning the particle properties and/or the driving force of self-assembly, one can control the size and dimensions of the resulting structures (Manoharan et al., 2003; Rycenga et al., 2008).

Although major progress has been made in three-dimensional microscopic self-assembly, observing the dynamic behaviour during the assembly process remains a challenge due to the small size and time constants involved. Several approaches have been explored to model and simulate these processes (Grant et al., 2011; Whitelam and Jack, 2015; Zhang et al., 2005). However, these approaches rely on exhaustive Monte-Carlo simulations, scaling unfavourably with the number of particles involved.

Magnetic forces have been used extensively as driving forces in self-assembly on all scales, together with various sources of agitating energy. 
When exposed to an external magnetic field, it has been demonstrated that nanoscopic magnetic rods form bundles (Love et al., 2003) or multimers when driven by ultrasound (Tanaka et al., 2007). Although paramagnetic spheres form chains, they will form ribbon structures (connected, parallel chains) for chains exceeding 30 particles (Darras et al., 2016; Messina and Stankovic, 2017) and flower-like patterns result when magnetic and non-magnetic beads are mixed with ferrofluids (Erb et al., 2009). In the absence of an external magnetic field, a theoretical study of off-centred magnetic dipoles in spherical particles (Yener and Klapp, 2016) shows that lateral displacement of the dipoles results in structures that are more compact than chains. On millimetre-scales, magnetic forces and vibrations have been used to quickly and efficiently assemble particles with correct orientation on a template (Shetye et al., 2008, 2010). Templated self-assembly has further been studied by agitating particles levitated in a paramagnetic fluid (Ilievski et al., 2011a; Woldering et al., 2016). Also on centimetrescales, magnetic forces have been used to form particles rather than structures, such as the spontaneously folding elastomeric sheets with embedded electronics; as demonstrated by Boncheva et al. (2005). Lash et al. (2015) showed that polystyrene beads self-assemble into HCP packed structures by solvent evaporation. Larger polystyrene particles $(>18 \mu \mathrm{m})$ required additional disturbing energy (ultrasonic energy) as a disturbing energy source to self-assemble. Macroscopic self-assembly processes on a centimetre scale are dominated by twodimensional structures, where mechanical shaking is the most widely used source of disturbing energy.

Hacohen et al. (2015) demonstrated DNA-inspired patterned bricks with embedded magnets, self-assembling into a programmed structure, but report gravity bias. Stambaugh et al. (2003) reported self-assembled 2D structures of centimetre-sized spherical particles with internal magnets that were shaken vertically, and observed different resulting structures that were based on particle concentration and magnet shape. Ilievski et al. (2011b) demonstrated self-assembly of centimetre-sized magnetic cubes into chains in a turbulent flow by submerging them in a rotating reactor filled with water, this way introducing eddy flows as a disturbing energy. They also introduced the concept of effective temperature, describing the motion of particles as if Brownian by nature. Even though the assembly process is three-dimensional, the resulting structures are limited to a single dimension and the dynamics involved are not studied.

To build upon this work, we introduce an experimental setup, which is designated "macroscopic self-assembly reactor", as a simulator for microscopic selfassembly. In this reactor, we study the motion and interaction of centimetresized objects. Particles are subject to a downward gravitational force and a drag force that is created by an upward water flow. We chose the particle density to balance these forces, causing them to appear weightless. Following Ilievski et al. (2011b), we use a turbulent water flow as an agitating source, simulating the Brownian motion on a microscopic scale. We employ permanent magnets, resulting in attraction forces between the particles.

By increasing particle size from micrometres to centimetres, not only the 
ease of observation but also the characteristic time constants increase decidedly. This makes the self-assembly process visible using conventional cameras. As a result of scaling up the system, the environment also changes; laminar flows become turbulent while inertia effects become dominant. At the same time, Brownian motion becomes negligible. Therefore, it is crucial to study to what extent the macroscopic system is a good simulator for microscopic environments, which is the main topic of this publication.

In this chapter we characterise the motion and dynamics of particles in a macroscopic self-assembly reactor. By observing the trajectories of a single particle in the reactor, we quantify the similarity between Brownian motion of said dynamics. By observing the interaction of two particles in the reactor, we can characterise the most fundamental building block of the self-assembly process, which is the interaction of magnetic spheres in a turbulent environment.

\subsection{Theory}

Brownian motion is the apparent motion of microscopic particles suspended in a fluid or gas, resulting from collisions with their surrounding molecules. It can be characterised by a three-dimensional random walk. The nature of the environment in terms of flow patterns (laminar, turbulent) is characterised by the Reynolds number (Landau and Lifshitz, 1987):

$$
\operatorname{Re}=\frac{\rho v L}{\mu},
$$

where $\rho\left[\mathrm{kg} \mathrm{m}^{-3}\right]$ is the density of the fluid/gas, $v\left[\mathrm{~m} \mathrm{~s}^{-1}\right]$ the velocity of the fluid/gas with respect to the object, $L[\mathrm{~m}]$ a characteristic diameter and $\mu\left[\mathrm{kg} \mathrm{m}^{-1} \mathrm{~s}^{-1}\right]$ the dynamic viscosity of the fluid/gas. Low and high numbers (loosely speaking for $\operatorname{Re}<1$ and $\operatorname{Re}>5000$ ) correspond to respectively laminar and turbulent flow.

\subsubsection{Diffusion}

A random walk has an average square displacement that increases linearly as time increases. We can define a diffusion constant $D\left[\mathrm{~m}^{2} \mathrm{~s}^{-1}\right]$ which, in a system with three degrees of freedom, links average displacement $\left\langle x^{2}\right\rangle\left[\mathrm{m}^{2}\right]$ to time $t$ [s] according to

$$
\left\langle x^{2}\right\rangle=6 D t
$$

This model holds only if the average distance travelled is much smaller than the size of the container in which the particles move. In our experiment this is not the case and, therefore, container geometry needs to be taken into account.

To account for the confined space, we first consider a particle performing a random walk along a single dimension. The particle displacement with respect 
to its starting location after $t$ seconds is normally distributed with variance $\sigma_{x}^{2}=2 D t$. Hence, the average squared displacement $\left\langle x^{2}\right\rangle$ is equal to the variance of the distribution. The probability of the particle being outside of the confined space is zero. To account for this effect, we replace the normal distribution by a truncated normal distribution. If the truncation is symmetrical on both tails of the normal distribution, $x_{\mathrm{t}}[\mathrm{m}]$, then the truncated distribution is given by

$$
n_{\mathrm{t}}\left(x, \sigma, x_{\mathrm{t}}\right)= \begin{cases}\frac{n(x, \sigma)}{N\left(x_{\mathrm{t}}, \sigma\right)-N\left(-x_{\mathrm{t}}, \sigma\right)} & -x_{\mathrm{t}} \leq x \leq x_{\mathrm{t}} \\ 0 & \text { otherwise, }\end{cases}
$$

where $n(x, \sigma)$ is the normal distribution and $N(x, \sigma)$ is the cumulative normal distribution. The average squared displacement of a confined particle is the variance of this distribution:

$$
\left\langle x^{2}\right\rangle=\sigma^{2}\left(1-\frac{x_{\mathrm{t}} n\left(x_{\mathrm{t}}, \sigma\right)}{N\left(x_{\mathrm{t}}, \sigma\right)-\frac{1}{2}}\right) .
$$

For $x_{\mathrm{t}} / \sigma \gg 1$, the particle does not yet experience the confinement. In this situation $n\left(x_{\mathrm{t}}, \sigma\right) \approx 0$ and $\left\langle x^{2}\right\rangle=\sigma^{2}$. For $x_{\mathrm{t}} / \sigma \ll 1$ the chance of finding the particle in the container is uniformly distributed $\left(n_{\mathrm{t}}=1 / 2 x_{\mathrm{t}}\right)$, and $\left\langle x^{2}\right\rangle$ saturates at $x_{\mathrm{t}}^{2} / 3$.

When moving to three dimensions, the average squared displacement of the separate dimensions can be simply summed because they are orthogonal.

The diffusion coefficient can only be determined if there has been a sufficient amount of collisions. In between the collisions, particles have constant velocity and direction. Due to the stochastic nature of the collision events, the velocity autocorrelation decays exponentially with time constant (Langevin, 1908; Lemons and Gythiel, 1997)

$$
\tau_{\mathrm{v}}=\frac{m^{*}}{f}
$$

where $f\left[\mathrm{~kg} \mathrm{~s}^{-1}\right]$ is the drag coefficient and $m^{*}[\mathrm{~kg}]$ is the effective mass.

The situation for $t \ll \tau_{\mathrm{v}}$ is referred to as the ballistic regime. Here, the average squared distance travelled $\left\langle x^{2}\right\rangle$ is quadratic rather than linear in time. The transition from the (quadratic) ballistic regime to the (linear) diffusion regime (eq. 2.2) is modelled phenomenologically by:

$$
\sigma^{2}=6 D \frac{t^{2}}{t+\tau_{\mathrm{v}}}
$$

Note that both the effective mass $m^{*}$ and the drag coefficient $f$ depend on the environment. The effective mass takes into account the fact that when the particle is accelerated, the surrounding water mass is also accelerated. For incompressible fluids with either zero viscosity or infinite viscosity (Stokes flow), the added mass is $50 \%$ of the mass of the water displaced by the sphere (Landau and Lifshitz, 1987). For turbulent flow, both experiment (Pantaleone and Messer, 
2011) as well as numerical simulations (Chang and Maxey, 1994, 1995) show that the added mass is also to a good approximation $50 \%$, irrespective of the Reynolds number or acceleration. There are reports that the added mass might be bigger in cases where the sphere is traveling through its own wake (Odar and Hamilton, 1964), which is rare in our experimental setup. Therefore, we have suggested a simple estimate of the added mass (Landau and Lifshitz, 1987),

$$
m^{*}=m+\frac{2}{3} \pi r^{3} \rho_{\text {fluid }},
$$

for a particle with radius $r[\mathrm{~m}]$ and mass $m[\mathrm{~kg}]$ surrounded by a fluid with density $\rho_{\text {fluid }}\left[\mathrm{kg} \mathrm{m}^{-3}\right]$.

\subsubsection{Velocity distribution}

Li et al. (2010) have experimentally proven that the velocity of particles performing a Brownian motion is Maxwell-Boltzmann (M-B) distributed. This distribution of velocity $v\left[\mathrm{~m} \mathrm{~s}^{-1}\right]$ is determined by its mode $v_{\mathrm{p}}$,

$$
p(\nu)=\frac{4 v^{2}}{\sqrt{\pi} v_{\mathrm{p}}^{3}} e^{-\left(\frac{v}{\nu_{\mathrm{p}}}\right)^{2}} .
$$

At the mode, the distribution reaches its maximum; thus $v_{\mathrm{p}}$ is the most probable velocity. For completeness, we note that the average squared velocity is $\left\langle v^{2}\right\rangle=\frac{3}{2} v_{\mathrm{p}}^{2}$.

\subsubsection{Drag coefficient}

Brownian motion is primarily studied on the microscopic scale, where the Reynolds number is much smaller than unity. In this case, the drag force is linear in velocity and the relevant drag coefficient $f$ is equal to the Stokes drag coefficient. However, on a macroscopic scale, we deal with turbulent flow and a high Reynolds number, where the drag force $F_{\mathrm{d}}[\mathrm{N}]$ is quadratic in velocity (Landau and Lifshitz, 1987),

$$
F_{\mathrm{d}}=\frac{1}{2} \rho_{\text {fluid }} C_{\mathrm{d}} A v^{2} \text {, }
$$

where $C_{\mathrm{d}}$ is the drag coefficient and $A\left[\mathrm{~m}^{2}\right]$ is the cross sectional area of the object in the direction of motion.

In our experiment, the particles are continuously "falling" through the upward water flow. This upward flow is set to the terminal velocity $v_{\mathrm{t}}$ of the particles, so that they levitate in front of the camera. Assuming that the changes in the velocity of the particle caused by turbulence are much smaller than the terminal velocity, we can obtain an effective drag coefficient by linearising around the terminal velocity

$$
f=\left.\frac{\mathrm{d} F_{\mathrm{d}}}{\mathrm{d} \nu}\right|_{\nu=v_{\mathrm{t}}}=\rho_{\text {fluid }} C_{\mathrm{d}} A v_{\mathrm{t}} .
$$




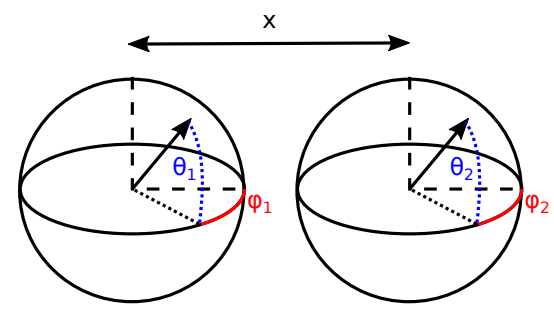

FIGURE 2.1 - The interaction between two spheres modelled by magnetic dipoles

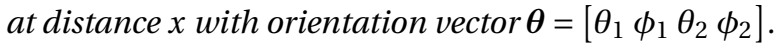

\subsubsection{Disturbing energy}

On the micro-scale, the diffusion coefficient and velocity distribution of particles in the fluid can be linked to the temperature. This concept can be extrapolated to macro-scale systems where disorder is achieved by shaking rather than by temperature. In that case, one speaks about effective temperature (Ilievski et al., 2011b; Wang and Wolynes, 2011), which is usually significantly higher than the environmental temperature. Since shaking can be highly directional, we prefer to characterize the shaking action by energy $(k T[\mathrm{~J}])$ rather than temperature to avoid confusion.

Starting from the velocity distribution (eq. 2.8), and considering that $\left\langle v^{2}\right\rangle=$ $3 \mathrm{kT} / \mathrm{m}$ for three-dimensional random walks, the most probable velocity is related to the kinetic energy through:

$$
k T=\frac{1}{2} m^{*} v_{\mathrm{p}}^{2} .
$$

The Einstein relation also relates the diffusion constant and viscous drag coefficient of a particle to the thermal energy $k T$ :

$$
k T=f D .
$$

If particles in a self-assembly reactor behave according to Brownian motion, both relation (2.11) and (2.12) can be used to obtain the disturbing energy and should give identical results.

In addition to measuring the disturbing energy $k T$ from Brownian motion, we can also estimate it from the interaction between two attracting magnetic objects. In this situation, we use the fact that he probability of the system being in a state is governed by M-B statistics (Feynman et al., 1970). Consider a system of two spherical magnetic particles in a confined space (Figure 2.1). The chance that the distance of those particles measured from center-to-center is smaller than $x_{0}$ is: 


$$
\begin{aligned}
p\left(x \leq x_{0}\right) & =\frac{1}{Z} \int_{d}^{x_{0}} \int_{\boldsymbol{\theta}} g_{\mathrm{r}}(x) e^{-\frac{E_{\mathrm{m}}(\boldsymbol{\theta}, x)}{k T}} d \boldsymbol{\theta} d x \\
Z & =\int_{d}^{D} \int_{\boldsymbol{\theta}} g_{\mathrm{r}}(x) e^{-\frac{E_{\mathrm{m}}(\boldsymbol{\theta}, x)}{k T}} d \boldsymbol{\theta} d x \\
\boldsymbol{\theta} & =\left[\theta_{1} \phi_{1} \theta_{2} \phi_{2}\right] .
\end{aligned}
$$

Here $g_{\mathrm{r}}(x)$ is the probability density function of a sphere pair with distance $x$ between their centres, unaffected by magnetic forces, which models the influence of the geometry of the reactor.

The distance between the cylindrical magnets is at all times at least a factor four of the magnet height $h(h \leq d / 4)$. At this point, we approximate their magnetic field as well as their magnetic moments by point dipoles. This approximation is accurate within $1.3 \%$ for our magnet geometry. In that case, the magnetic energy of particle 1 with magnetic moment $\boldsymbol{m}\left(\theta_{1}, \phi_{1}\right)\left[\mathrm{A} \mathrm{m}^{2}\right]$ in a field $\boldsymbol{B}\left(\theta_{2}, \phi_{2}, x\right)[\mathrm{T}]$ generated by particle 2 reduces to

$$
E_{\mathrm{m}}(\boldsymbol{\theta}, x)=-\boldsymbol{m}\left(\theta_{1}, \phi_{1}\right) \cdot \boldsymbol{B}\left(\theta_{2}, \phi_{2}, x\right) .
$$

Equation (2.13) can be approximated numerically by a Monte-Carlo approach in which a large number of random combinations of sphere locations and orientations are selected, yielding different values for $E_{\mathrm{m}}$. The geometry factor $g_{\mathrm{r}}$ is approximated by repeated random sampling of two point locations in a confined geometry and then gathering statistics about their distance.

\subsection{Materials and methods}

\subsubsection{Reactor}

The experimental setup consists of a transparent cylinder with an inner diameter of 17.3(1) cm containing the particles of interest (Figure 2.2). Gravity is counteracted by pumping water from the bottom to the top via four $4.0(1) \mathrm{cm}$ diameter inlet holes using a MAXI.240T pump (PSH pools). The water exiting the cylinder is collected in an open container connected to the pump inlet. The water flow entering the pump is monitored using an altometer (IFS 4000, Krohne Messtechnik $\mathrm{GmbH})$.

Meshes spaced at $17 \mathrm{~cm}$ prevent the particles from moving outside the field of view of cameras placed around the reactor. The dynamics of the particle-fluid system are determined by the particle density and geometry, as well as water flow speed and its degree of turbulence.

At flow speeds of approximately $30 \mathrm{~cm} \mathrm{~s}^{-1}$ and a water temperature of $20^{\circ} \mathrm{C}$ the system is characterised by Reynolds numbers of 57000 and 61000 for respectively the reactor cylinder and the inlet tubes. This is more than an order of 

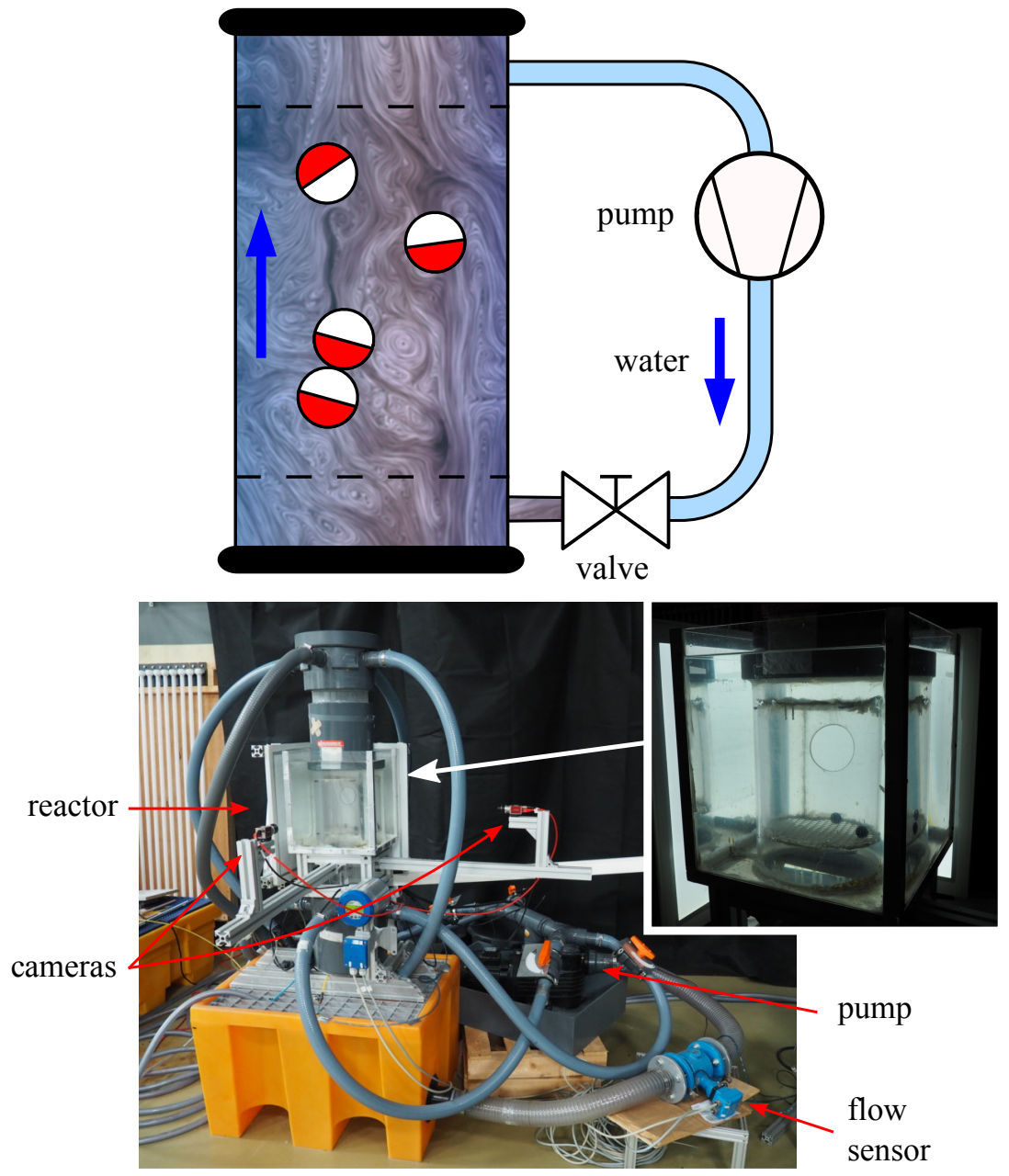

FIGURE 2.2 - Schematic (top) and experimental (bottom) setup of the macroscopic self-assembly reactor. Water is pumped from the bottom to the top of the reactor, counteracting gravity and supplying energy to the particles via turbulent flow. Meshes prevent the particles from moving outside of the field of view of cameras placed around the reactor.

magnitude larger than 2040, the lowest number which can support turbulence in a tube (Avila et al., 2011). The turbulence generated by the tubing, the disruptive nature of the inlet area and the meshes is supported by this environment. 


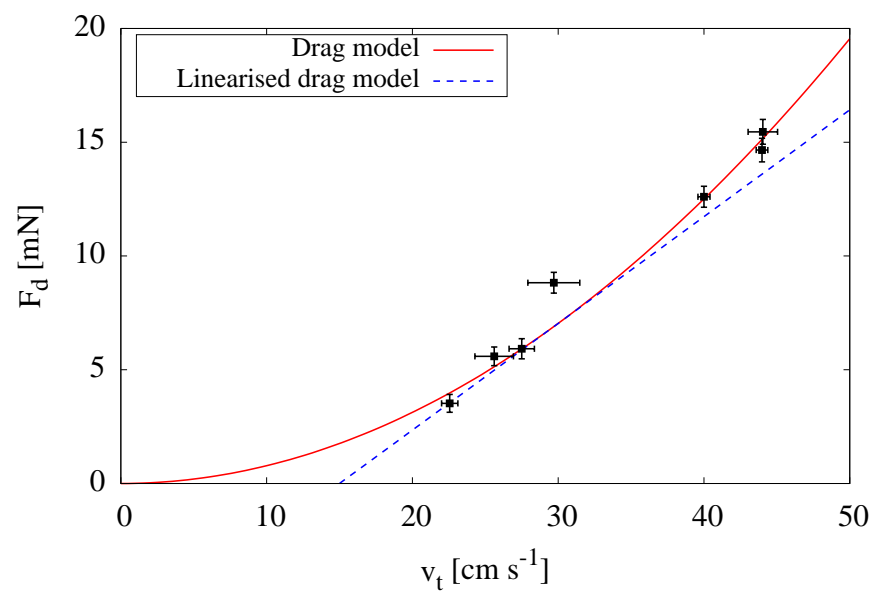

FIGURE 2.3 - Calculated drag force versus measured terminal velocity for spheres with equal diameter but varying densities. The effective drag coefficient is obtained by linearisation around the terminal velocity (eq. 2.10), illustrated by the blue dashed line for $v_{t}=30 \mathrm{~cm} \mathrm{~s}^{-1}$.

\subsubsection{Particles}

The particles used in the experiments are 3D-printed polymeric (ABS) spheres with a diameter of $1.67(1)$ to $2.02(2) \mathrm{cm}$ and a corresponding density of $1.33(2)$ to $1.25(4) \mathrm{g} \mathrm{cm}^{-3}$ (larger particles have lower density). The core of the spheres consist of cylindrical, axially magnetised $\mathrm{NdFeB}$ magnets with a length of 3.80(5) $\mathrm{mm}$ and a diameter of 3.80(5) $\mathrm{mm}$ (Supermagnete, grade N42, Webcraft $\mathrm{GmbH})$. The dipole moment $\left(50.8(1) \mathrm{mA} \mathrm{m}^{2}\right.$ ) was determined by measuring the force between two magnets using a balance.

The drag coefficient of the particles was estimated from their terminal drop velocity. For this, particles with a range of densities but identical diameter of $1.85 \mathrm{~cm}$ were released at the top of a $2 \mathrm{~m}$ high cylinder filled with water. Once an equilibrium between drag- and gravitational force had been established (approximately $0.5 \mathrm{~m}$ after release), the velocity of the particles was measured with a video camera over a distance of $1.0 \mathrm{~m}$. Figure 2.3 shows the measured relation between drag force and terminal velocity. From fitting equation 2.9, we obtain $\frac{1}{2} \rho_{\text {fluid }} C_{\mathrm{d}} A=78(3) \mathrm{g} \mathrm{m}^{-1}$. Assuming the density of water to be $1000 \mathrm{~kg} \mathrm{~m}^{-3}$, we obtain $C_{\mathrm{d}}=0.58(2)$. Spheres of this diameter and velocity in water have a Reynolds number of approximately 5500. At this value, Brown and Lawler (2003) predict $C_{\mathrm{d}}=0.39$, which is substantially lower. The reason for the discrepancy is unknown to us. The measured drag coefficient is used in the remainder of this thesis. 


\subsubsection{Reconstruction}

Two calibrated, synchronised cameras (Mako G-131, Allied Vision) were placed around the reactor at an angle of approximately $90^{\circ}$ and they recorded datasets at $30 \mathrm{fps}$ at a resolution of $640 \times 512$. The reactor is surrounded by a square, water-filled aquarium to prevent refraction due to its cylindrical nature. Backlight panels were used to enhance contrast. Single spheres were observed for $15 \mathrm{~min}$ and two spheres for $30 \mathrm{~min}$. Offline, the location of the spheres was automatically detected using a custom written MATLAB script. A method based on the direct linear transform algorithm (Hartley and Zisserman, 2004) was used for $3 \mathrm{D}$ reconstruction, giving an average reconstruction error of $0.16 \mathrm{~cm}$. Trajectories closer than $1.5 \mathrm{~cm}$ to the meshes were discarded to rule out the significant effect of the altered hydrodynamic interaction at these interfaces. The velocity vector of the particle is obtained by $\mathbf{v}=\Delta \mathbf{x} f_{\text {cam }}$, the product of the particle displacement between two frames and the camera frame rate.

\subsection{Results}

\subsubsection{Single particles}

Figure 2.4 shows a set of reconstructed trajectories of a $1.85 \mathrm{~cm}$ sphere in the reactor. Each trajectory starts and ends when respectively exiting and entering the areas within $1.5 \mathrm{~cm}$ of the meshes, and is indicated by a different color.

Figure 2.5 shows the velocity calculated from these trajectories. The histogram is obtained from the absolute velocity (10600 data points) of a $1.80 \mathrm{~cm}$ sphere. A M-B distribution was fitted to the data by minimising the maximum distance $E_{\max }$ between the cumulative empirical and cumulative M-B distribution, yielding fitting parameter $v_{\mathrm{p}}$. A Kolmogorov-Smirnoff (K-S) test was used to quantify the quality of fit and to obtain a significance level Q to disproof the null hypothesis that the two distributions are the same (Press et al., 1992). With a $E_{\max }$ of 0.0073 and a $\mathrm{Q}$ of 0.70 , we have good reason to assume that the velocity is M-B-distributed.

Figure 2.6 displays the resulting $v_{\mathrm{p}}$ for spheres of various diameters, for which we find a range from 15.92 to $17.54 \mathrm{~cm} \mathrm{~s}^{-1}$. The fit to the M-B distribution has a $Q$-value above 0.05 for five out of the seven measurements. Even though the data suggests a slight decrease of velocity with increasing sphere size, the particle velocity fits very well to a model assuming constant velocity, with an average of $16.6(2) \mathrm{cm} \mathrm{s}^{-1}$. This analysis was carried out using a chi-square fitting routing, yielding the reduced $\chi^{2}$ error metric (ideally being around 1) and the corresponding Q-value (the probability that a $\chi^{2}$ equal or greater than the observed value is caused by chance) (Press et al., 1992). The reduced $\chi^{2}$ of this fit is close to unity (0.68) with a very high $Q$-value of 0.67 .

Figure 2.7 shows the normalised distribution of the particle at several $z$ slices across the reactor. It can be seen that the particle has a preference for the bottom area, especially near the reactor walls of the positive $x$-coordinate. We 


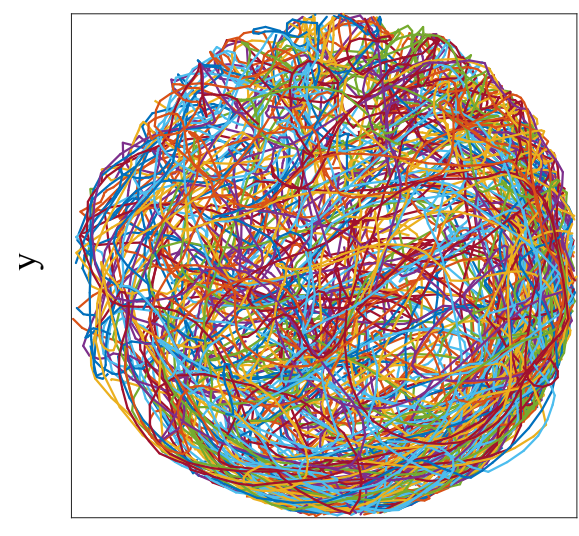

$\mathrm{X}$

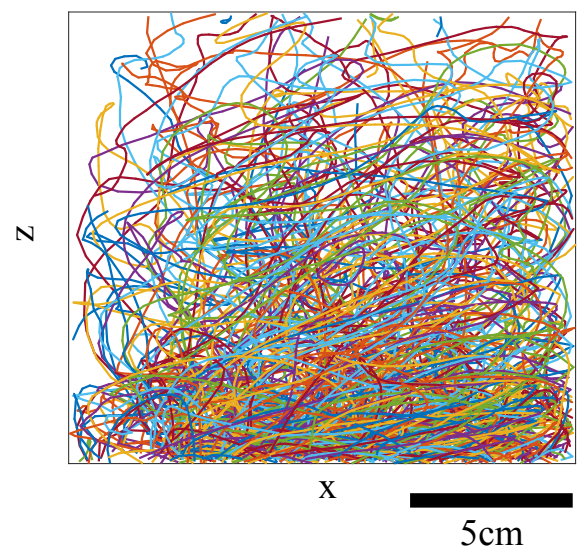

FIGURE 2.4 - Top (upper) and side (bottom) view of the reconstructed trajectories of a single sphere (diameter $1.85 \mathrm{~cm}$ ) moving through the reactor. Coordinates less than $1.5 \mathrm{~cm}$ close to the top-and bottom meshes are removed to rule out significant influence of the meshes. In this way, the single trajectory is cut into many smaller ones, which are each assigned a different color.

believe that this phenomenon is caused by a non-uniform flow pattern of water that results from the specific valve settings. These observations are analogous to a multi-temperature environment in a system of micro-particles; as particles are biased towards a state of minimum energy, they are more likely to be in areas with lower thermal energy.

The average squared displacement was calculated from the longest trajectories; that is, those with a minimum duration of $2.0 \mathrm{~s}$. Figure 2.8 shows the resulting curve for a sphere with a diameter of $1.90 \mathrm{~cm}$. The curve shows a quadratic regime below $0.3 \mathrm{~s}$, shortly entering an approximately linear regime before 


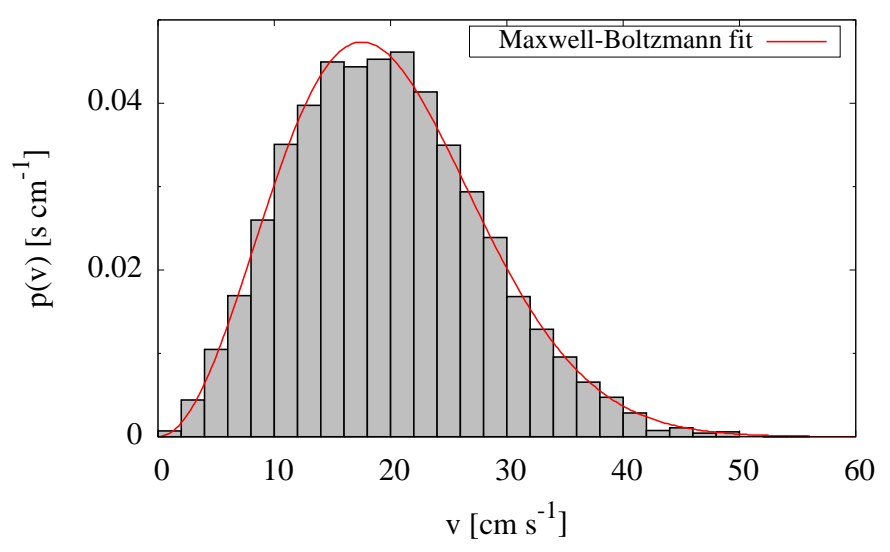

FIGURE 2.5 - Maxwell-Boltzmann (M-B) distribution fitted to the measured velocity distribution of a particle with a diameter of $1.80 \mathrm{~cm}$. The Kolmogorov-Smirnov (K-S) test quantifies a maximum distance between the theoretical and experimental cumulative distributions of 0.0073 with a $Q$ value of 0.70 , indicating a high probability that the velocity is indeed $M-B$ distributed.

slowly converging to a horizontal asymptote.

The movement of the sphere is in the quadratic, or ballistic, regime when the measurement time is shorter than the average time between directional changes ("collisions"), $\tau_{\mathrm{v}}$. Using measured values for the drag coefficient and effective mass in equation 2.5, we obtain values for $\tau_{\mathrm{v}}$ ranging from 134 to 149(10) ms. The saturation measured for longer observations is caused by the confined geometry of the reactor and will change as the reactor is changed in shape and size.

The model described by equations 2.4 and 2.6 was fitted to the measurements, yielding values for diffusion coefficient $D$ and average reactor size $x_{\mathrm{t}}$.

We have to take into account that the model has its limitations. First of all it is based on a symmetrical truncated normal distribution. This would require the particle to always start in the center of the reactor. In contrast, all of the measured trajectories start at a random place at the top or bottom of the reactor due to the method that we used to obtain separate trajectories.

Secondly, the cylindrical geometry of the reactor is not included in the model. These two issues mainly affect the estimation of the reactor size.

Finally, the ballistic regime was phenomenologically modelled without physical background. This region, which has a high weight factor during fitting the model to the data (due to the small error bars in the data), can result in a significant fitting error. 


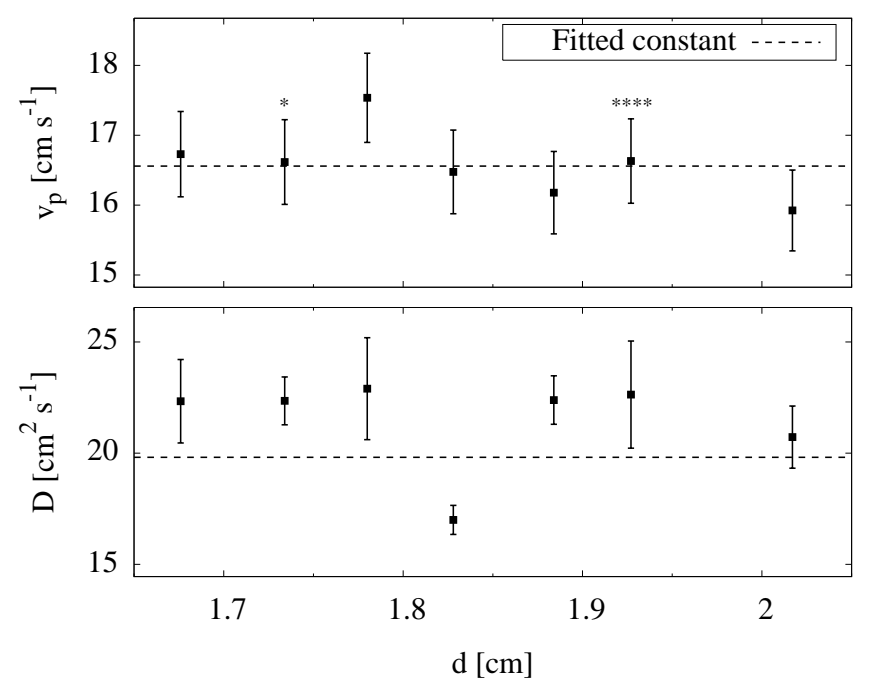

FIGURE 2.6 - Top: Mode of the M-B distribution obtained by fitting to the measured velocity distribution of particles of various diameters (reduced $\left.\chi^{2}=0.68, Q=0.67\right)$. Stars indicate the quality of fit ( $Q$-value) of the K-S test $\left(^{*}<0.05,{ }^{* * *}<0.0001\right)$. Bottom: Diffusion coefficient obtained by fitting the diffusion model to the average square displacement (reduced $\chi^{2}=5.85$, $\left.Q=4 \cdot 10^{-6}\right)$.

Given that only the latter aspect could give errors in the estimation of $D$, we consider the obtained values for $D$ to be quite reasonable, with values between 17 and $23 \mathrm{~cm}^{2} \mathrm{~s}^{-1}$ (see figure 2.6). The average diffusion coefficient for all of the measured diameters is $20(1) \mathrm{cm}^{2} \mathrm{~s}^{-1}$. Judging from the graph, there seems to be no reason to assume that the diffusion coefficient has a strong dependence on sphere diameter. It should be noted, however, that this assumption leads to a very high reduced $\chi^{2}(5.85)$ and low quality of fit $Q\left(4 \cdot 10^{-6}\right)$. However, due to the previously mentioned model inaccuracies, we think that we may have underestimated the errors in the estimation of $D$.

\subsubsection{Two-sphere results}

From the two-sphere experiments, the distance $x$ between the particles was tracked over time. Figure 2.9 shows the cumulative probability of sphere distance $p\left(x \leq x_{0}\right)$ for spheres of various diameters. Spheres with smaller diameters have a lower magnetic energy in connected state and, therefore, a higher probability of being connected. In other words, $p(x \leq d)$ becomes larger for smaller $d$. All of our measurements follow a similar profile: they consist of a curved regime for $x \leq 3 \mathrm{~cm}$ followed by an approximately linear region for 


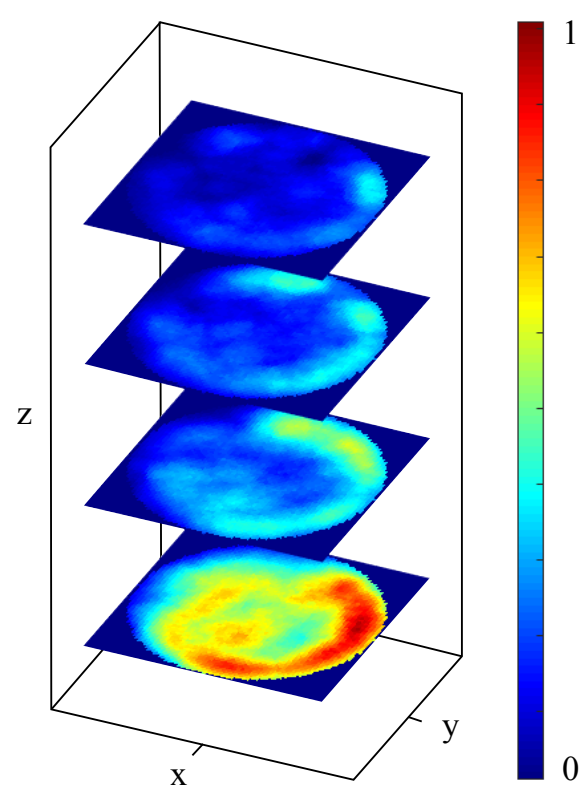

FIGURE 2.7 - Normalised probability distribution of a single sphere (diameter $1.85 \mathrm{~cm}$ ) in the reactor, displayed in slices along the reactor tube. The particle has a clear preference for the bottom region as well as the edge regions.

Quantified, the particle has a chance of $62 \%, 48 \%$ and $26 \%$ to be in, respectively, the right (positive $x$-coordinate), back (positive $y$-coordinate) and top (positive $z$-coordinate) halves of the reactor.

$x>3 \mathrm{~cm}$. The linear regime indicates that magnetic forces are no longer significant for particle interaction. For $x>13 \mathrm{~cm}$ there is a saturation effect caused by the reactor geometry. The model of equation 2.13 has been fitted to the curves by minimising the maximum distance between the curves (based on the Kolmogorov-Smirnoff method (Press et al., 1992)). Although this is not an exact fit, it manages to capture the shape with a maximum error of $5 \%$ of the full range.

\subsubsection{Disturbing energy}

The experiments provide three methods for the characterisation of the equivalent thermal energy of the system. Numerical values for the kinetic energy were calculated from the measured velocity and added mass according to equation 2.11. The measured diffusion coefficient and drag coefficient at the set water flow speed (equation 2.10) were used to calculate the energy using the Einstein relation (equation 2.12). Additionally, two-particle experiments provide 


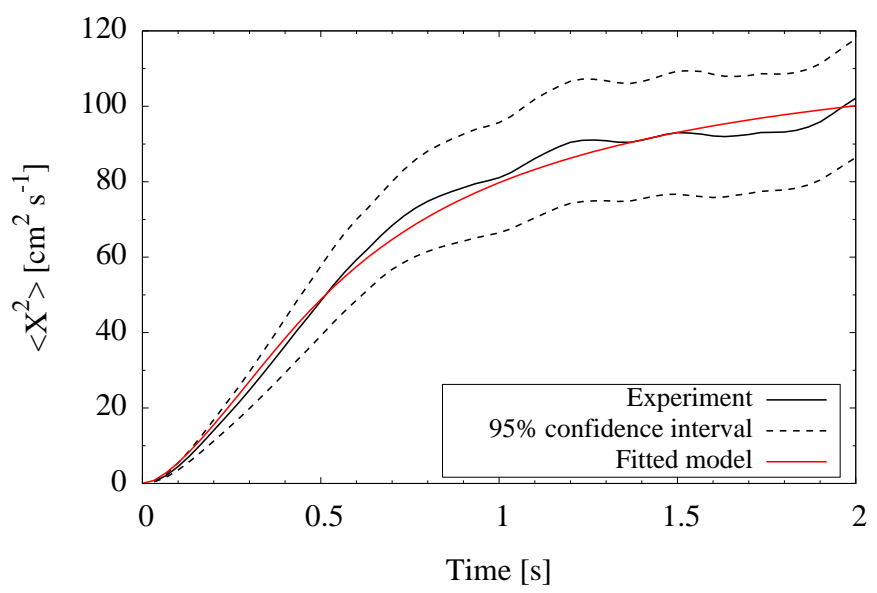

FIGURE 2.8 - Average squared displacement as a function of time for a sphere with diameter $1.90 \mathrm{~cm}$, calculated from 65 trajectories. The model fits within the $95 \%$ confidence interval.

numerical values for the equivalent energy as a result of fitting equation 2.13 to the measured data, as depicted in figure 2.9.

The resulting values for all of the spheres are summarised in figure 2.10. A first observation is that the results obtained via single sphere experiments (velocity, diffusion) are in the same order of magnitude, and differ approximately $20 \mu \mathrm{J}$. They span a range from approximately 60 to $120 \mu \mathrm{J}$. These values are, however, more than a factor of ten higher than the results obtained via the two sphere experiments, which range from approximately 6 to $7 \mu \mathrm{J}$. The possible origin for this discrepancy is discussed in the following section.

In all cases, the energy increases as the sphere size increases, by approximately $17 \%, 41 \%$, and $46 \%$ for, respectively, two-sphere experiments, diffusion, and velocity. As we concluded previously, the diffusion coefficient and average sphere velocity do not depend on the sphere size (figure 2.6). The increase of energy is caused by an increase in mass and friction coefficient, and both are dependent on sphere radius.

\subsection{Discussion}

From the trajectory analysis of single particles, we were able to determine that their velocity distribution closely follows a M-B distribution. Additionally, we have seen that the average squared displacement as a function of time follows a shape that was predicted by a confined random walk model. These conclusions strongly support the hypothesis that particles in the reactor perform a random 


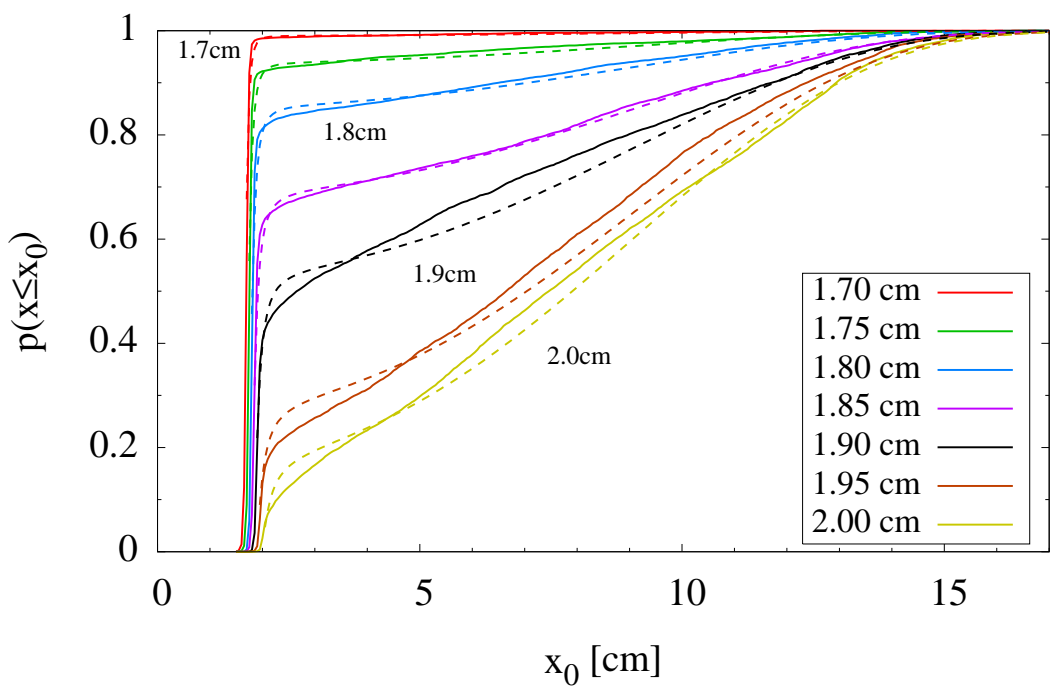

FIGURE 2.9 - Measured probability (cumulative) of the distance between the centres of two magnetic spheres ( $x$ ) for various sphere diameters. A model based on $M-B$ statistics captures the shapes of the curves with a maximum error of $5 \%$ of the full range. As the spheres decrease in size, they are more likely to be in a connected state.

walk.

When increasing the particle size, the observed disturbing energy $k T$ also increases. However, there is no observable increase in velocity or diffusion coefficient. For the energy calculated via velocity and diffusion, this means that this increase in energy is caused by an increase in, respectively, effective particle mass and drag coefficient. The corresponding curves, as shown in figure 2.10, are very similar due to the fact that the particle mass and drag force are coupled. With an increase in particle radius, both the mass and surface area are increased. The increase in energy occurs without physically changing the nature of the disturbing energy; that is, the speed and turbulence of the water flow is unaltered. This means that the amount of energy that is transferred from the environment to the particle is dependent on the particle geometry.

An explanation for this effect might be found in the wavelength dependence of the turbulence. Turbulence is introduced as a large wavelength disturbance at the bottom of the cylinder, after which it propagates upwards in an energy cascade that transfers the energy to smaller wavelengths. This process is dissipative (Richardson cascade (Richardson, 1922)). The resulting energy spectrum drops off at increasing wave numbers (Kolmogorov, 1941). Therefore, we can 


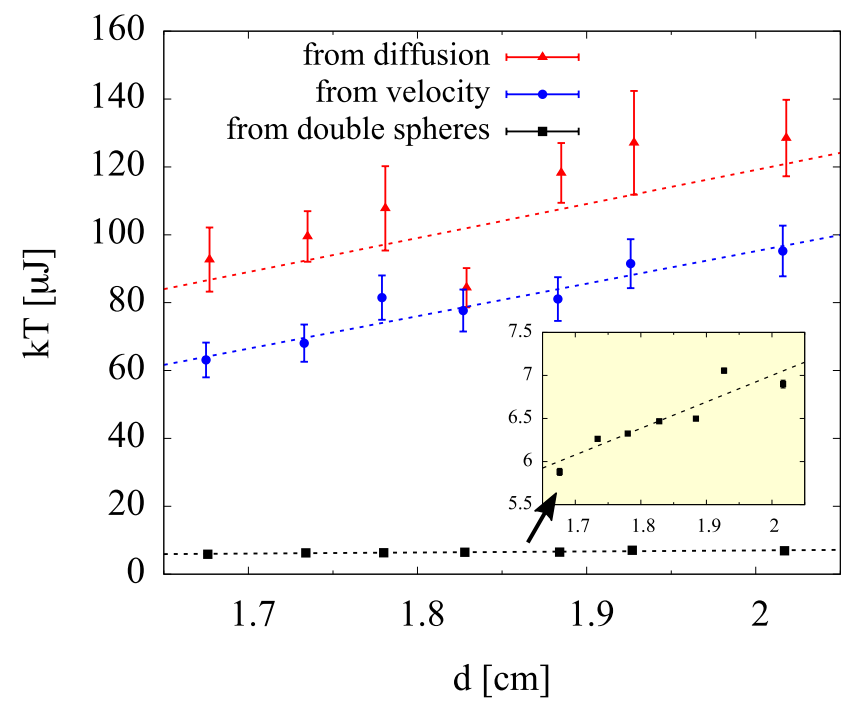

FIGURE 2.10 - Disturbing energy of the turbulent field calculated from the diffusion coefficient, the velocity distribution and double sphere experiments. The disturbing energy estimated from the single sphere experiments (diffusion, velocity) are approximately a factor 10 higher than that estimated from double sphere experiments. The dashed lines are guides to the eye. There is an increase in energy with an increase in sphere diameter, which is proportional with the increase in mass and friction coefficient.

assume that the disturbing energy as experienced by the particles is not, like in Brownian motion, characterised by a flat spatial frequency spectrum (white noise) but instead drops off at shorter wavelengths. So, effectively, the bandwidth of the energy transfer increases for larger particles.

The assumption of a dissipative energy cascade could also explain why the energy obtained from two-sphere experiments is lower compared to single sphere experiments. While all of the spatial frequency components in the turbulent flow drive an object around the system in a random walk, wavelengths in order of the particle diameter contribute most effectively to separation of connected particles. The disturbing energy dropping with decreasing wavelength would explain why the disturbing energy estimated from the two particle experiment is smaller than that obtained from the random walk.

It is perhaps in the spatial frequency spectrum where the analogy between turbulent flow and true Brownian motion breaks down. Therefore, we will need to characterise the effective energy of the system separately for particles of different size. Special care needs to be taken for large clusters of particles because they are effectively a large particle and, therefore, subject to a higher energy por- 
tion. At the same time, particle-particle interaction is subject to a lesser amount of disturbing energy. Consequently, such systems will have a bias towards the occurrence of smaller particle clusters.

\subsection{Outlook}

Successful self-assembly is characterised by the ability of the system to end up in a desired end-state, generally the global energy minimum. This will require an interplay in assembling and disturbing forces which assist the system by removing itself from local energy minima. The experimental results have proven that particles in the reactor show a Brownian-like motion and that the disturbing turbulent field is able to separate otherwise connected particles. This gives confidence that multi-particle systems will be able to explore the energy landscape and that the results have significance for similar processes taking place on the micro-scale.

To demonstrate the possibilities of using this experimental setup for further studies, we loaded the reactor with six spheres with embedded magnets. Figure 2.11 shows several stills of a video in which the spheres form different structures, thereby exploring the energy landscape. The highest energy state is found when all of the spheres are disconnected. The energy of the system decreases with the number of connections made, so a six sphere chain structure (top right) has a lower energy than two three-sphere structures (bottom left). One more bond can be created by forming a six-sphere ring (center right). For structures with more than four spheres, the ring is the minimum energy state (Messina et al., 2014). Indeed, three-sphere rings are hardly ever observed. By long term observation, one could measure the relative occurrence of the different structures and check if they agree with M-B statistics.

\subsection{Conclusions}

We have constructed an experimental setup that allows us to study the connection dynamics of centimeter-scale objects by analysing the interaction of magnetic attraction forces and disturbing turbulent forces. This "macroscopic self-assembly reactor" serves as a physical simulator of self-assembly processes on the microscale and nanoscale, allowing easy observation by drastically increasing both the length and time scales.

Trajectory analysis of single spherical particles shows that they perform a random walk, which analogous to Brownian motion. Spheres with diameters ranging from 1.7 to $2.0 \mathrm{~cm}$ have a range of velocities that are $\mathrm{M}-\mathrm{B}$ distributed. The most probable velocity (mode) is independent on sphere size and has a value of $16.6(2) \mathrm{cm} \mathrm{s}^{-1}$. The average square displacement over time, or the 'diffusion profile,' fits to a confined random walk model. The diffusion coefficient appears to be independent of sphere size, with an average value of $20(1) \mathrm{cm}^{2} \mathrm{~s}^{-1}$. 

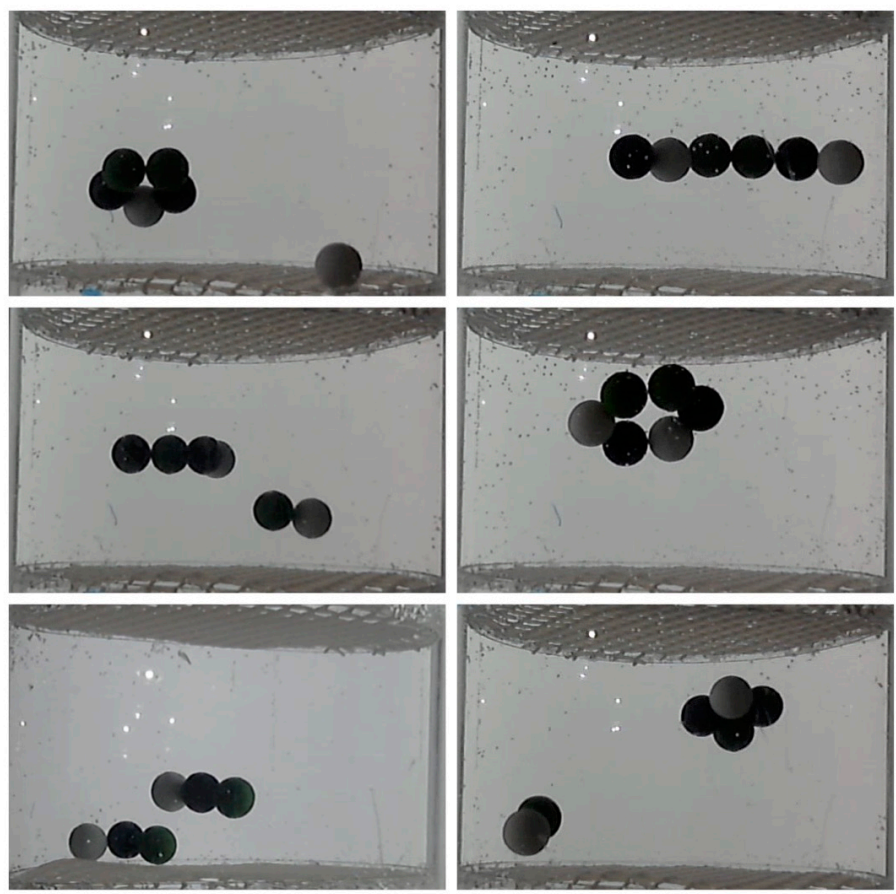

FIGURE 2.11 - Multi-particle systems show to explore the energy landscape, ending up in both local and global energy minima.

Although statistical analysis disproves this statement, we believe that the measurement error has been underestimated.

The particle distribution is non-uniform over the reactor. The particle is, for instance, three times as often in the bottom half of the reaction compared to the top half. Although this non-uniform distribution does not affect the Brownian motion behaviour, it virtually reduces the reactor size.

In two-particles systems, we observe self-assembly dynamics; that is, the particles occasionally connect and disconnect. The cumulative distribution of the distance between the centers of the particles fits with a maximum error of $5 \%$ of the full range of the distribution to a model based on M-B statistics.

The disturbing energy (analogue to temperature) of the reactor was estimated from the velocity distribution and diffusion (single particle experiments), as well as from the dynamic interaction of two-particle systems. The estimates of the disturbing energy determined from single sphere experiments are in the same order of magnitude. However, the disturbing energy obtained from twosphere experiments is at least one order of magnitude lower (approximately $6.5 \mu \mathrm{J}$ compared to $80 \mu \mathrm{J}$ ). From this we can conclude that for self-assembly 
studies, the disturbing energy of the system cannot be calibrated from single sphere experiments alone.

The disturbing energy increases with increasing sphere diameter, from 1.7 to $2.0 \mathrm{~cm}$. For the single sphere experiment, this increase is more prominent ( $41 \%$ via diffusion analysis, $46 \%$ via velocity analysis) than for the two-sphere experiment $(17 \%)$. We reason that the energy transfer from the turbulent environment to the particles is dependent on particle size and geometry.

In addition to the two-sphere experiment, periodic connection and disconnection events have also been observed for a six-sphere system, forming ringand line-based structures. This demonstrates that the reactor can be successfully applied to study self-assembly processes at convenient length and time scales, and it may be a good simulator for microscopic environments. 


\title{
Chapter 3
}

\section{Control of disturbing energy in self-assembly}

\begin{abstract}
Turbulence can be used as a source of disturbing energy in macroscopic self-assembly. It is a macroscopic equivalence to thermal energy $k T$ on the microscale. The amount of turbulence can be adjusted by changing the input flow into the reaction chamber. We measured the effect of an increase in turbulence on particle diffusion, velocity and disturbing energy. In our experimental setup, we can vary the disturbing energy by a factor of eight. Since we use upward flow to avoid sedimentation, there is a directional dependency in the motion of the particles. A region exists in which this asymmetry is minimal. This study shows that one can tune the disturbing energy analogue to a change in temperature at the microscale, which increases the relevance of macroscale self-assembly studies for the self-assembly processes of micro- and nano-particles.

The work in this chapter is a team effort. My contribution includes composing the theoretical background, building and programming and calibrating the dual-camera system and performing the data analysis. I wrote the software for particle detection, 3D-reconstruction, particle tracking and trajectory analysis and performed the statistical analysis. The reactor was constructed in Twente by Remco Sanders and Léon Woldering and was modified by Per Löthman, who also designed the particles and did the experiments. Gayoung Kim also carried out several experiments.
\end{abstract}

\subsection{Introduction}

In a self-assembly process, order seems to appear from disorder spontaneously. Particles self-assemble due to their mutual attraction and the disturbing energy in the environment. In macroscopic self-assembly the mutual attraction or assembling energy is frequently magnetic (Gross and Dorigo, 2008), and the disturbing energy turbulent flow (Ilievski et al., 2011b; Murugesan et al., 2015; 
Roland et al., 1992; Zheng et al., 2004). Turbulence is inherent random and chaotic and resembles the microscopic thermal motion. Thermal operations such as slow or rapid cooling or heating of liquids, solids or gases can be realized analogously by rapid regulation of the turbulent flow. Figure 3.1 shows excerpts of a video recording of macroscopic self-assembly of twelve polymer spheres of $2 \mathrm{~cm}$ diameter with embedded permanent magnets in a self-assembly reactor. The structure formation is clearly dependent on the degree of turbulence; at maximum turbulence the spheres are disconnected and start to form structures as turbulence decreases. At low turbulence the minimum energy structure (ring) is formed. This is a macroscopic representation of microscopic quenching or cooling sequence and demonstrates the paths of self-assembly nicely. In this paper we describe a method to adjust turbulence and we systematically characterize the effect of turbulence on velocity distributions and diffusion of particles in the turbulent flow. To the best of our knowledge, this is the first time systematic investigations of turbulence as a disturbing energy were made.

\subsection{Theory}

The analysis of particle trajectories and two particle interaction were introduced in chapter 2 . Here, we also analyse the diffusion coefficient and velocity distribution for the projection of the particle movement on the vertical axis $(z)$, along the main direction of the flow, and in the horizontal plane perpendicular to the flow $(x, y)$. The diffusion of a particle in a confined space was described in chapter 2 for one dimensional movement along a line segment. If the particle motion along the three projections is uncorrelated, we can apply the same expression for the average squared displacement,

$$
\left\langle x^{2}\right\rangle=\sigma_{x}^{2}\left(1-\frac{x_{\mathrm{t}} n\left(x_{\mathrm{t}}, \sigma_{x}\right)}{N\left(x_{\mathrm{t}}, \sigma_{x}\right)-\frac{1}{2}}\right) .
$$

where $n\left(x, \sigma_{x}\right)$ is the normal distribution and $N\left(x, \sigma_{x}\right)$ is the cumulative normal distribution. For $x$, we can substitute the $y$ or $z$ coordinate. $\sigma_{x}$ is the standard deviation of the displacement and the variance $\sigma_{x}^{2}$ can be related to the diffusion coefficient along a coordinate in one dimension by

$$
\sigma_{x}^{2}=2 D_{x} t
$$

In chapter 2, we used the Maxwell-Boltzmann distribution to the describe the distribution of the vectorial velocity, using a most probable velocity $v_{\mathrm{p}}$. The distribution of the velocity of the individual components is Gaussian distributed, described by the standard deviation $\phi$ of the velocity distribution:

$$
p\left(v_{x}\right)=\frac{1}{\sqrt{2 \pi \phi_{x}^{2}}} e^{-\frac{v_{x}^{2}}{2 \phi_{x}^{2}}},
$$

where again $x$ can be substituted with $y$ or $z$. 


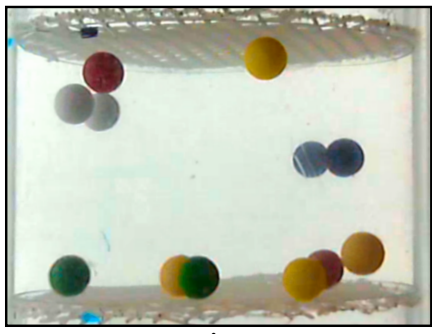

maximum

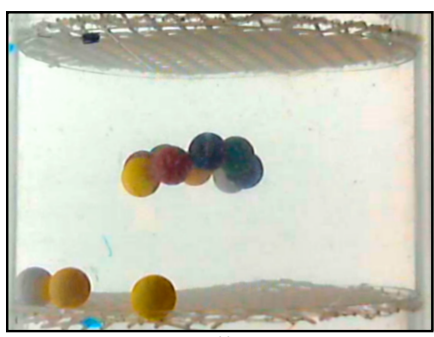

medium

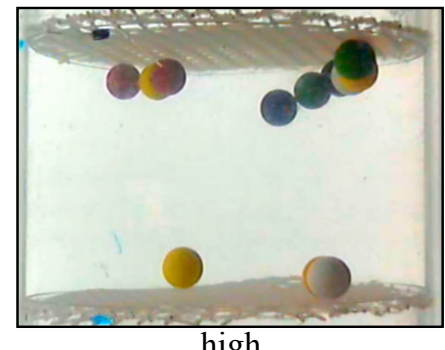

high

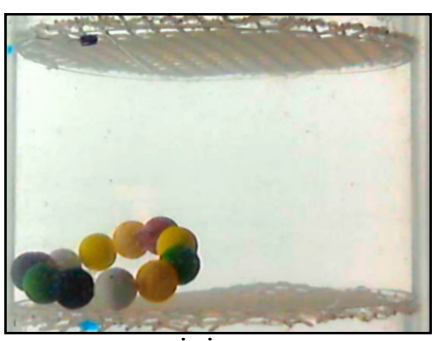

minimum

FIGURE 3.1 - The effect of degree of turbulence on the structure-formation of twelve magnetic polymer spheres that self-assemble in a vertical turbulent water flow. Decreasing turbulence leads to increased structure formation (lines and rings of different lengths and shapes). At maximum turbulence only single spheres appear whereas at minimum turbulence, the lowest energy structure ( $a$ 12-sphere-ring) appears. The local magnetic forces of each individual sphere interacts as the spheres explore the energy landscape in order to find the lowest-energy configuration.

\subsection{Methods}

The self-assembly reactor has been introduced in chapter 2 . The system has four inlet ports on the bottom of the cylinder. For this work, the inlet ports were equipped with valves. This allows us to inject the water flow asymmetrically and increase the turbulence. The valves are 2-way PVC ball valves (Type S6 DN40-14, $50 \mathrm{~mm}$ diameter, Praher Plastics Austria $\mathrm{GmbH}$ ).

A schematic front- and top-view of the reactor is shown in figure 3.2. The valves can be opened between $0^{\circ}$ (fully closed) and $90^{\circ}$ (fully open). Maximum turbulence can be achieved by opening one valve only (right position, indicated by $0^{\circ}$ ). For simplicity, we decided to adjust the remaining three valve identically, where $90^{\circ}$ is defined as fully open, so fully symmetric and minimum asymmetry.

The real self-assembly reactor and three representative valve settings (bottom) are displayed in figure 3.3. The valve settings in the bottom row correspond to the bottom schematic image in figure 3.2. 

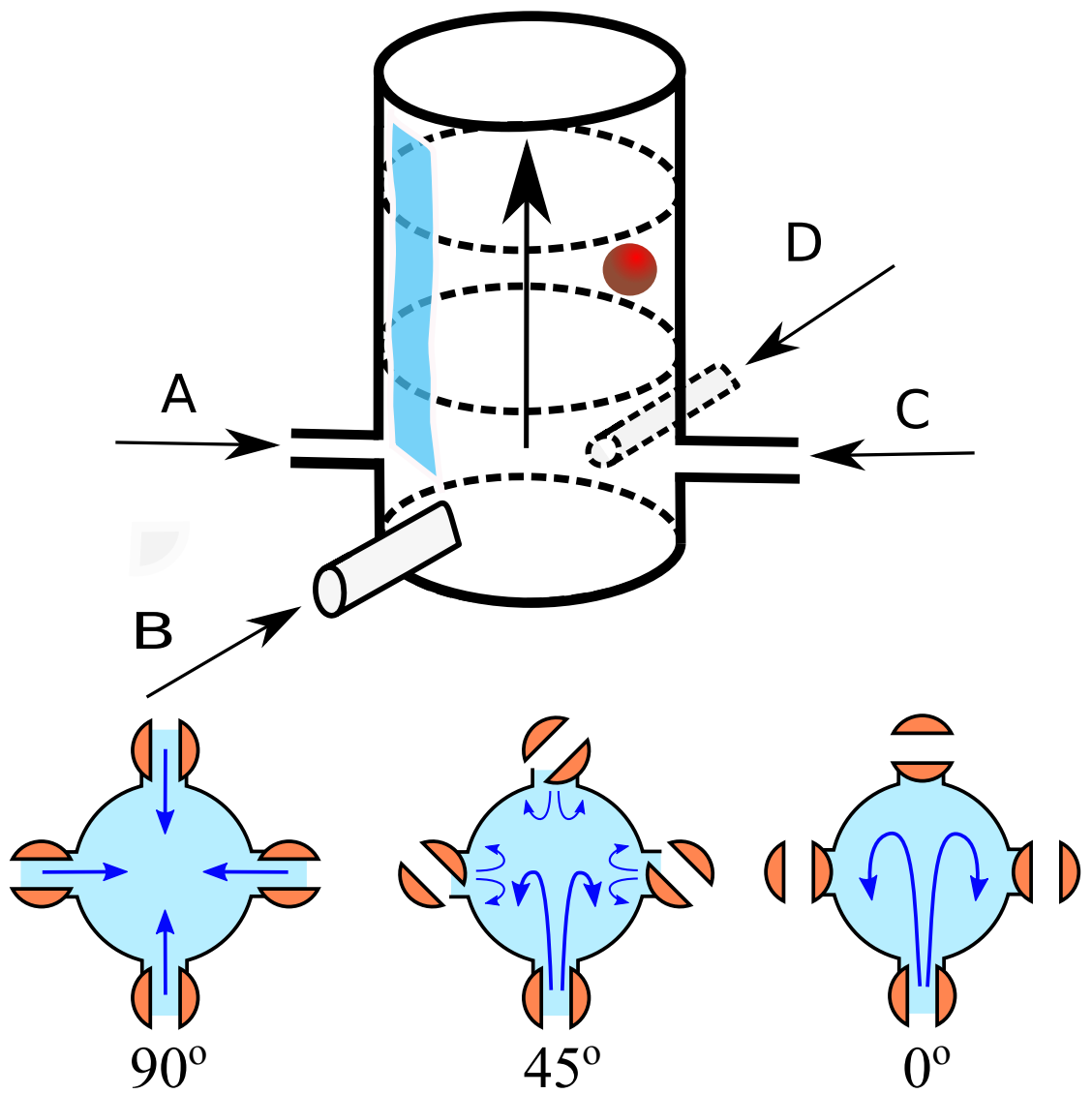

FIGURE 3.2 - Schematic front- and top-view of the self-assembly reactor. Four water inlets (A,B,C and D), a particle (red) and flow direction are shown in the front-view. The dotted circles in the middle of the reactor indicate the position of the nets that are used as placeholders for the particle(s). In the bottom-view, three valve settings are shown; minimum (left, $90^{\circ}$ valve opening), medium ( $45^{\circ}$ valve-opening) and maximum (right, $0^{\circ}$ valve-opening). One of the valves is kept open for all three settings which, in addition to the turbulent flow from the three closed valves, provides an asymmetry to the flow field. 


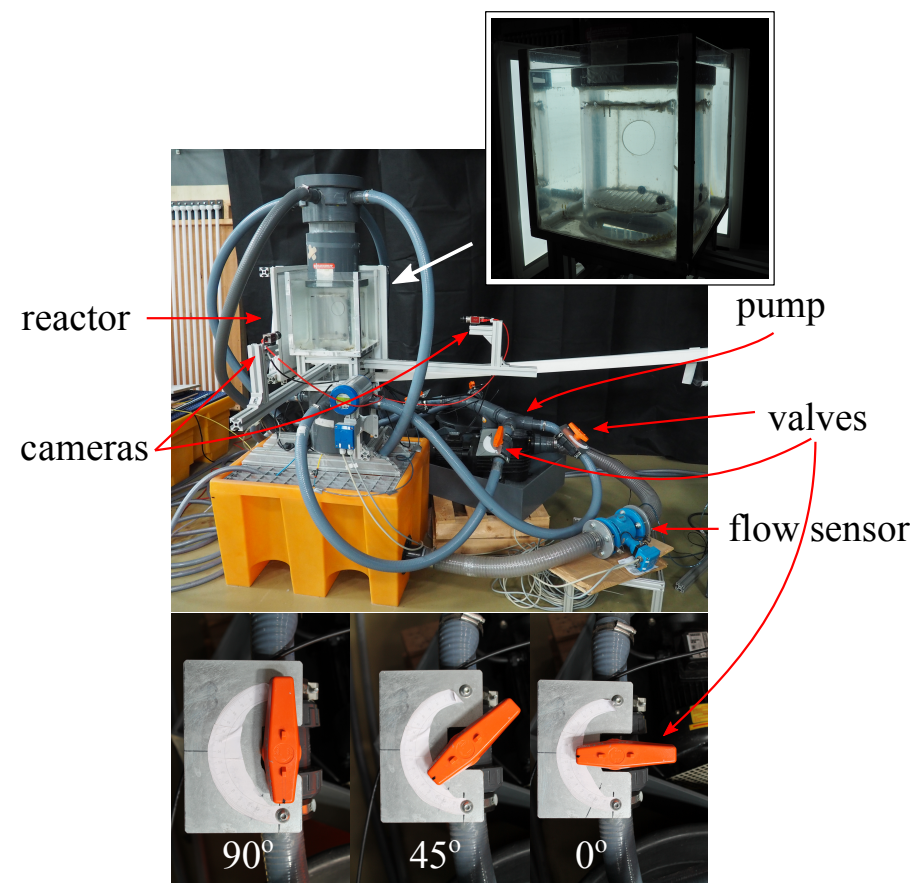

FIGURE 3.3 - Front-view of the self-assembly reactor. Four bottom-inlet tubes, valves, pump, cameras are shown. The inset shows a magnification of the actual reaction chamber. Below, three pictures of one of the three valves at different valve-opening, as in figure 3.2, corresponding to increasing degree of turbulence in the incoming water flow.

\subsubsection{Flow calibration}

We expect the turbulence in the cylinder to be proportional to the asymmetry in the inflow. Since we use ball-valves, the flow through the valves has a nonlinear relationship with the valve angle. Therefore we chose to convert valve angle to flow by measuring the flow through the cylinder as function of the valve opening, see figure 3.4. In this measurement, three valves are closed and one valve is opened over an angle $\theta$. We used maximum pump effort.

From this measurement we derive a dimensionless measure for the asymmetry of the flow:

$$
\text { flow asymmetry }=1-\frac{f(\theta)}{f\left(90^{\circ}\right)},
$$

where $f(\theta)$ is the water flow speed through the valves controlled during the experiments at opening angle $\theta$. This way, at minimum turbulence, when all 


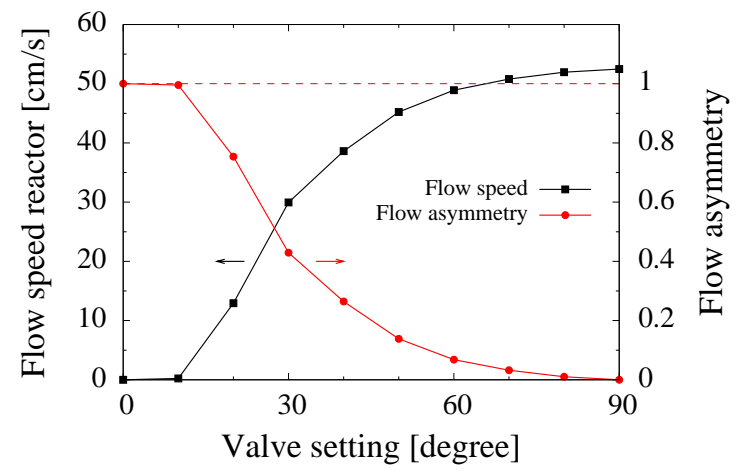

FIGURE 3.4 - Flow speed through the reactor at maximum pump effort as a function of the valve angle of one valve, where the other three remain closed.

valves are fully opened, the flow asymmetry is defined as 0 and at maximum turbulence, when three valves are closed, the flow asymmetry is defined as 1 .

\subsubsection{Particles}

The particles are identical as in chapter 2. They are 18.80(7) mm diameter polymer (ABS) spheres with a $3.80 \times 3.80(5) \mathrm{mm}$ cylindrical NdFeB permanent magnet placed in the center of each sphere.

\subsubsection{Reconstruction}

Two synchronized cameras were used for video recordings as described in chapter 2. Particles were observed in environments with different degrees of turbulence. For each setting, videos were recorded for $15 \mathrm{~min}$ for single sphere experiments and $30 \mathrm{~min}$ for two sphere experiments. Both the 3D trajectories of a single sphere and the $3 \mathrm{D}$ distance between two spheres were reconstructed via custom written MATLAB scripts.

\subsubsection{Measurement precision}

To determine the diffusion constant, the trajectory of the particle in the turbulent flow is observed as described in chapter 2. Each trajectory longer than $0.5 \mathrm{~s}$ is fitted to the diffusion model described in chapter 2 . These values are averaged for a large number of trajectories to obtain an estimate of the diffusion coefficient. The precision of the estimate increases with the number of measurements, which is expressed in the standard error (the standard deviation of the fit divided by the square of the number of fits). 


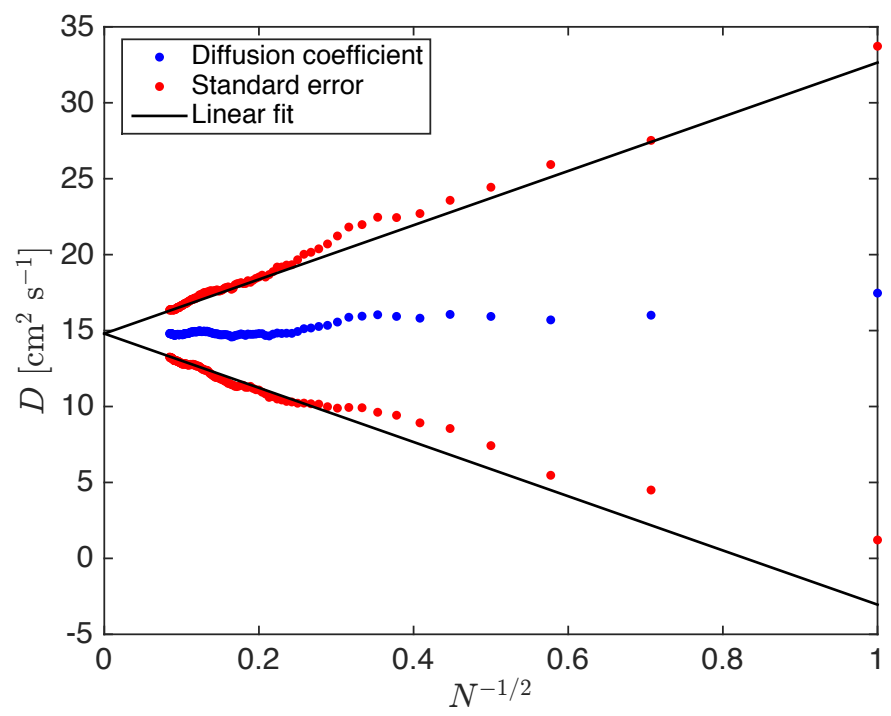

FIGURE 3.5 - The estimated diffusion coefficient (blue dots) and $1 \sigma$ confidence interval as a function of the inverse square root of the number of trajectories (N).

To validate this process, we determined the diffusion coefficient for sets of data with varying number of trajectories. The result is shown in figure 3.5 , where the blue dots represent the estimate of the diffusion coefficient as function of the number of trajectories $N$. As expected, the estimated value converges (to about $15 \mathrm{~cm}^{2} \mathrm{~s}^{-1}$ ) with increasing number of measurements. The red dots indicate the $1 \sigma$ confidence limit on the estimate (we are $68 \%$ confident that the diffusion coefficient lies between the red dots). By plotting the confidence interval as a function of $N^{-1 / 2}$, figure 3.5 shows that indeed the precision of the estimate increases with the square root of the number of trajectories.

From this measurement, we conclude that for a $1 \sigma$ confidence limit of $5 \%$ of the estimated value, we need at least 570 trajectories. We obtain approximately 80 trajectories of $0.5 \mathrm{~s}$ duration per minute. The total measurement time per experiment should therefore be at least $7 \mathrm{~min}$. To be on the safe side, the duration of the experiments in this study was $15 \mathrm{~min}$.

\subsection{Results}

We observed the movement of a single sphere and the interaction between two spheres in the reactor, and first determined the disturbing energy as a function of the flow asymmetry, applying the methods introduced in chapter 2 . We also investigated the directional dependence in the velocity distribution, which will 


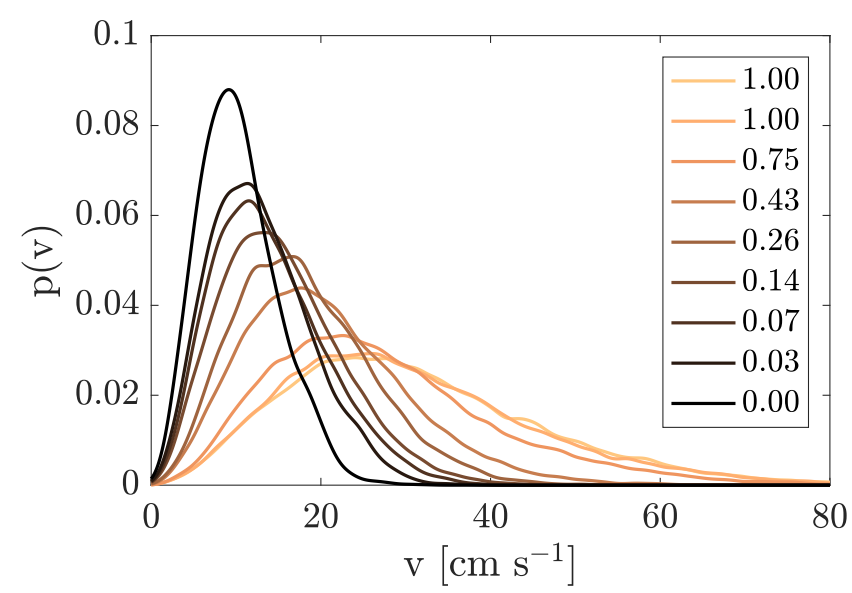

FIGURE 3.6 - Velocity distributions of a single particle for different flow asymmetry settings (legend) in the reactor show a Maxwell-Boltzmann-like distribution. The distribution was obtained via a kernel density estimation using a Gaussian kernel with $\sigma=1 \mathrm{~cm} \mathrm{~s}^{-1}$. Increased turbulence leads to a higher average velocity.

be discussed in section 3.4.2.

\subsubsection{Relation between flow asymmetry and disturbing energy}

We observed the influence of turbulence on the kinetic behaviour of a single particle in terms of the most probable speed $v_{\mathrm{p}}$ and its diffusion coefficient, as well as the interaction between two particles. From these observations, we can determine the relation between the flow asymmetry and the disturbing energy $k T$.

\section{Influence of flow asymmetry on velocity}

Figure 3.6 shows the velocity distribution of a particle in a turbulent flow for various settings of flow asymmetry. The graphs were obtained by a kernel density estimation using a Gaussian kernel with a standard deviation of $1 \mathrm{~cm} \mathrm{~s}^{-1}$. With increasing flow asymmetry there is an increase in particle velocity.

The velocity data was fitted to a Maxwell-Boltzmann distribution, as described in chapter 2 , with only the most probable speed $v_{\mathrm{p}}$ as fitting parameter. In figure 3.7 this most probably speed is shown as a function of flow asymmetry. This relation is approximately linear. Over the full range of available flow asymmetry, the velocity varies by a factor of three from approximately 10 to $30 \mathrm{~cm} \mathrm{~s}^{-1}$. 

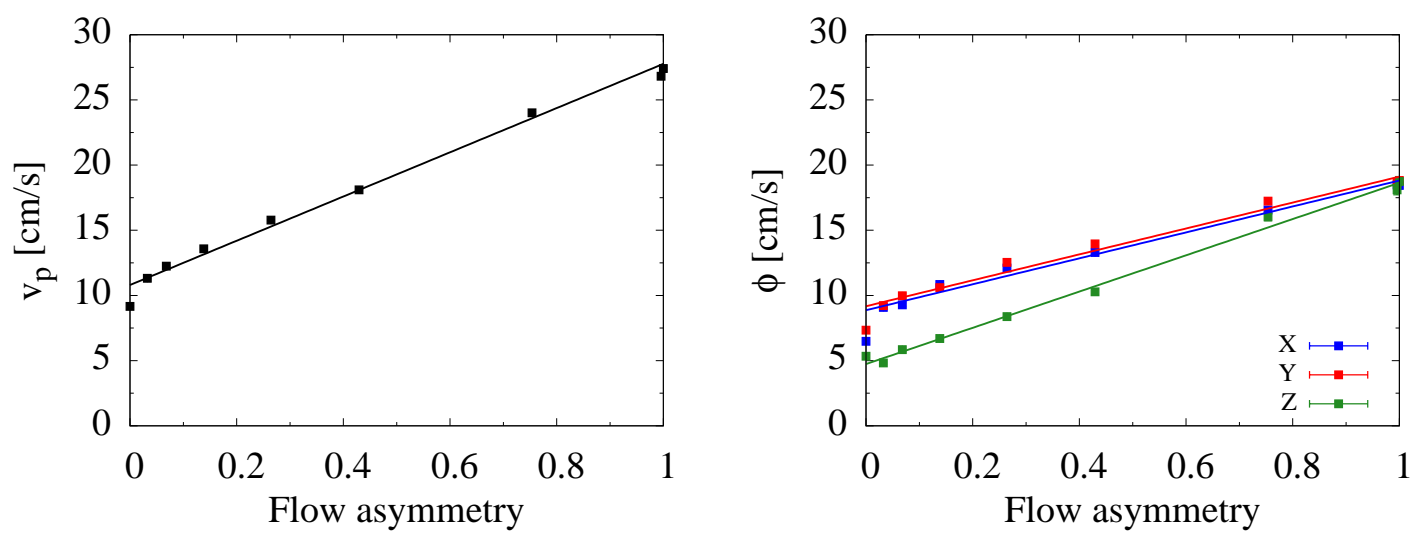

FIGURE 3.7 - Left: the most probable speed $v_{p}$ of the

Maxwell-Boltzmann-distributed particle velocity as a function of flow

asymmetry. The relation is approximately linear. The velocity increases by

almost a factor of three, indicating that the turbulence is increased. Right: the standard deviation of the horizontal $(x, y)$ and vertical $(z)$ components of the particle velocities as a function of flow asymmetry. The velocity in the vertical direction is significantly lower than in the horizontal direction for a flow asymmetry below 0.5 .

\section{Influence of flow asymmetry on diffusion coefficient}

The diffusion coefficient was estimated by fitting a confined random walk model to the measured average squared displacement, as in chapter 2 . The latter was obtained by averaging the squared displacement of trajectories with a duration of $2 \mathrm{~s}$. Figure 3.8 shows the diffusion coefficient as a function of flow asymmetry. As in the case of velocity, the diffusion increases roughly linear with flow asymmetry, now by a factor of six from approximately 7 to $44 \mathrm{~cm}^{2} \mathrm{~s}^{-1}$.

\section{Influence of flow asymmetry on disturbing energy $k T$}

As described in chapter 2, the velocity distribution as well as the diffusion coefficient of a single sphere can be related to disturbing energy, using the kinetic energy $k T=1 / 2 m^{*} v_{\mathrm{p}}^{2}$ and the Einstein relation $k T=f D$ respectively.

A third method for obtaining the disturbing energy can be obtained from particle interaction. When two particles are inserted in the reactor, they connect and disconnect intermittently. The ratio between the time they are connected and disconnected depends on their magnetic interaction energy and the disturbing energy in the system. In chapter 2 a method is described to extract this disturbing energy from the distribution of observed particle distances. 

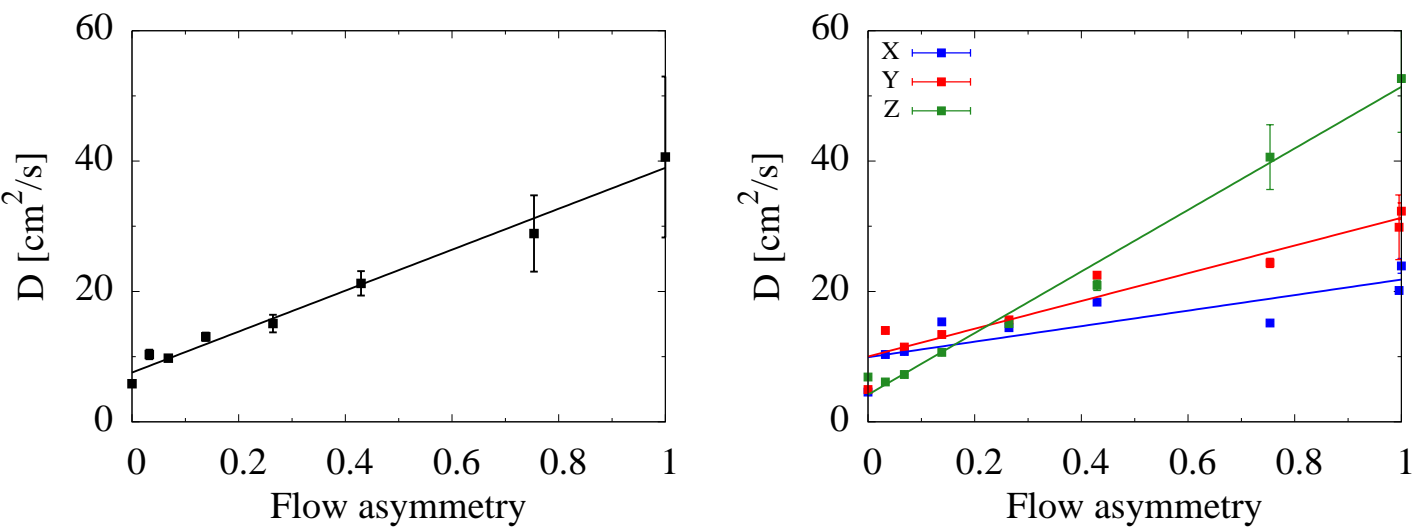

FIGURE 3.8 - Left: the diffusion coefficient of the motion of a single particle as a function of flow asymmetry. The diffusion coefficient increased approximately a factor six from minimum to maximum turbulence. The relation is roughly

linear. Right: the diffusion coefficient per dimension as a function of flow asymmetry. Above a symmetry of 0.5, the difference between the components is fairly large, but reduce significantly for lower turbulence. The estimation of $D$ underestimates its error bars, which for that purpose have been ignored for the fit.

This method is more precise and fundamentally more correct than the method based on connection-disconnection duration.

In figure 3.9, all estimates for the disturbing energy are plotted together. The relationship between $k T$ and flow asymmetry fit well to a linear function in all three cases. The estimates of the disturbing energy from the single sphere experiments are very similar, certainly considering the measurement error. However, like in chapter 2, these values are an order of magnitude higher than the values obtained from the two-sphere experiments. For both single and two sphere experiments the disturbing energy increases with increasing flow asymmetry. The increase is a approximately a factor two higher for the single sphere experiment (for the two single sphere experiment the increase $(a+b) / b=6(1)$, and for the double sphere, $(a+b) / b=3.1(7))$.

\subsubsection{Directional dependency of disturbing energy}

The water flow is directed from the bottom to the top in the reactor in order to counteract gravity acting on the particles. It is therefore expected that the vertical $(z)$ component of the particles motion deviates from the horizontal ( $x$ and $y$ ) components. Additionally, there might be an asymmetry in the $x y$ plane as well, since the flow is injected asymmetrically at high turbulence. These 

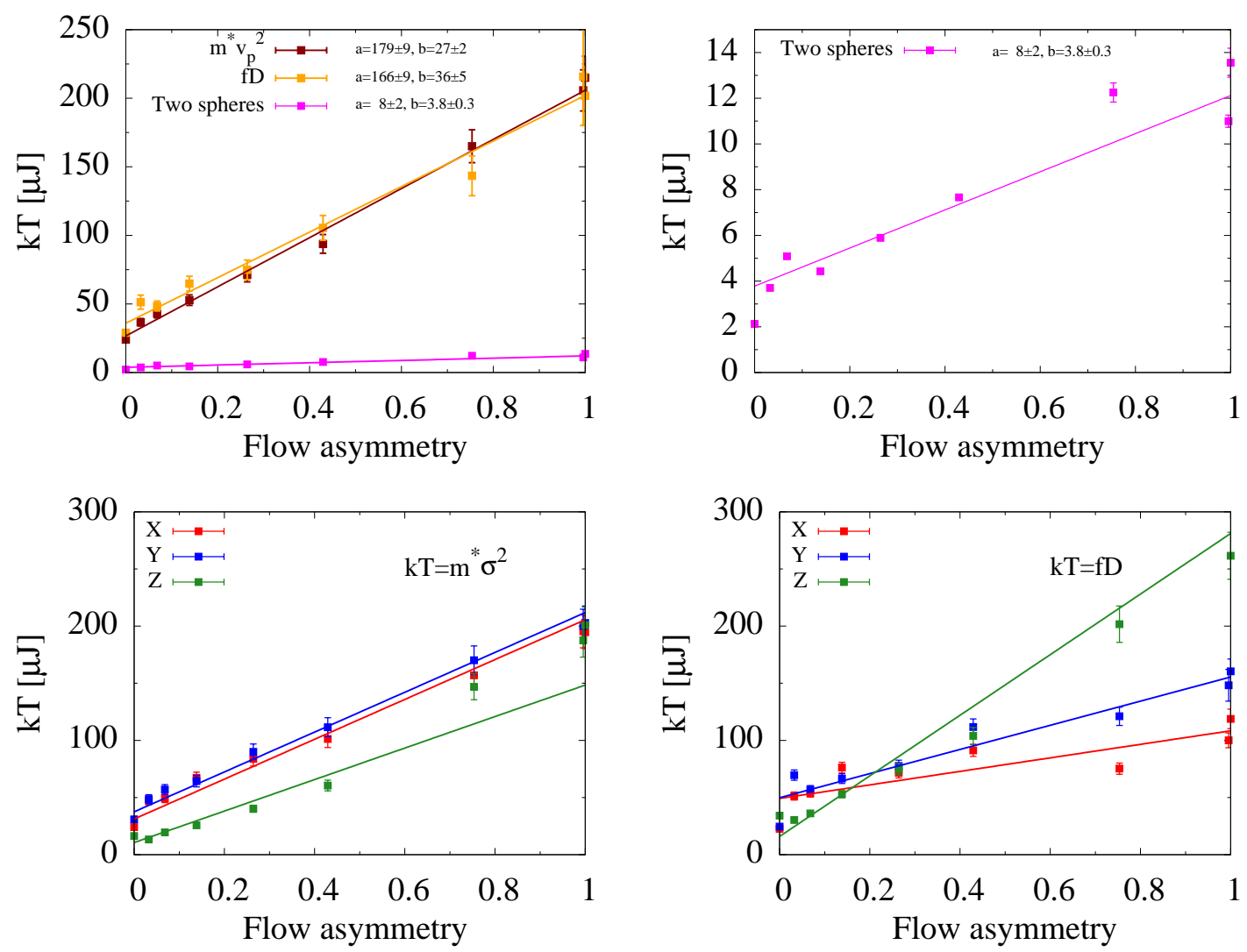

FIGURE 3.9 - The disturbing energy ( $k T$ ) increases approximately linear with flow asymmetry. Top left: determined from single sphere experiments, using the diffusion coefficient (Einstein relation $f D$ ) and most probable velocity $\left(m^{*} v_{p}^{2}\right)$ and from the interaction between two spheres. The disturbing energy determined from the velocity distribution agrees very well with that obtained from the diffusion coefficient. The values obtained from this single sphere experiments however are an order of magnitude higher than that of the two sphere experiment. Top right: Enlarged view of the energy determined via the interaction between two spheres. Bottom left: The directional dependence of the disturbing energy derived from the velocity. Bottom right: The directional dependence of the disturbing energy derived from the diffusion coefficient. Due to the fact that we underestimate the error on the diffusion coefficient, it is ignored by the linear fits for the Einstein relation. 
effects are present both in the velocity distribution as well as in the diffusion coefficient.

\section{Directional dependence velocity}

Figure 3.10 shows the velocity distribution of a particle in a turbulent flow for various settings of flow asymmetry. The graphs were obtained by a kernel density estimation using a Gaussian kernel with a standard deviation of $1 \mathrm{~cm} \mathrm{~s}^{-1}$. With increasing flow asymmetry, the velocity distribution becomes wider, such that the average absolute velocity increases. The velocity in the horizontal dimensions are similar, but the vertical velocity is significant lower for low asymmetry settings. According to to the theory a normal distribution (equation 3.3) was fitted to the measurements. The standard deviation $\phi$ is plotted in figure 3.7. There is no significant difference in the horizontal direction ( $x$ and $y$ components), and there seems to be no correlation of the difference with flow asymmetry. For flow asymmetry below 0.5 the velocity in the $z$-direction is significantly lower, up to a factor of two.

\section{Directional dependence diffusion coefficient}

Figure 3.8 shows the diffusion coefficients along the three different directions. Even though the data is scattered, the values for the horizontal dimensions only differ moderately. The diffusion coefficient in the $z$-dimension, however, shows a much stronger dependence on flow asymmetry, diving below the horizontal components for low flow asymmetry and vice versa.

\section{Directional dependency of disturbing energy $k T$}

As before, the disturbing energy can be derived from the velocity and diffusion coefficients, but now for the individual $x$-, $y$ - and $z$-components $\left(k T=m^{*} \phi^{2}\right.$ and $k T=f D$, respectively). Figure 3.9 (bottom) shows the estimated values of the disturbing energy. For clarity, two graphs are plotted, one of the estimate based on kinetic energy (bottom left) and one for the estimate based on the Einstein relation (bottom right). Of course these graphs show similar trends as figures 3.7 and 3.8, as the particle mass and friction coefficient do not change between the measurements; the velocity and diffusion coefficient fully determine the shape of these curves.

Like with the velocity analysis, there is no significant difference in kinetic energy per dimension for higher flow asymmetry. For asymmetry below 0.5 , however, the energy in the vertical component is much lower than that of the horizontal components with approximately a factor of factor two.

The results from the Einstein relation are significantly different; the energy scales different for the separate dimensions; the horizontal components are close but the vertical component has almost a factor of two more energy for high flow asymmetry settings. 

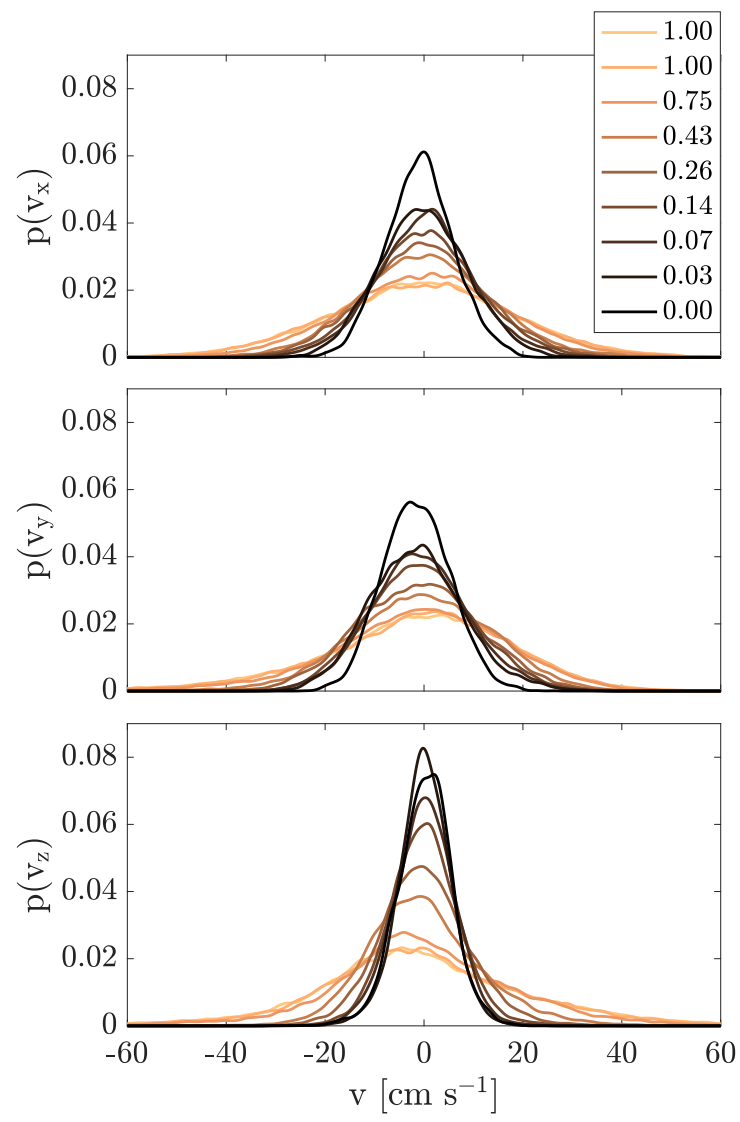

FIGURE 3.10 - The $x, y$ and $z$ components of the velocity of a single particle for different flow asymmetry settings (legend) in the reactor show a Gaussian-like distribution. The distribution was obtained via a kernel density estimation using a Gaussian kernel with $\sigma=1 \mathrm{~cm} \mathrm{~s}^{-1}$. Increased turbulence leads to a higher average velocity. The $z$ component velocity is significantly lower than that of the other dimensions. 


\subsection{Discussion}

The experiments clearly show that the particle velocity, diffusion coefficient as well as the disturbing energy increase with turbulence. When three of the four inlet valves are gradually closed, the inflow becomes more asymmetric and turbulence increases. This process adds to the turbulence created by the reactor geometry.

Creating asymmetry in the inlet flow is a practical way to change turbulence and mimic temperature changes on the micro- and nano-scale. The analogy between turbulent motion and thermal fluctuation is quite intriguing. There are however at least two area's where the analogy between turbulence and thermal fluctuation does not hold: directionality and spatial frequency power density.

\subsubsection{Directionality in turbulent flow}

The experiments show that one cannot ignore the directionality of the turbulent flow field. In analogy with temperature fluctuation, we would have to conclude that the temperature in the system is directionally dependent.

Judging from the observations on directional dependence, increase in turbulence has a more pronounced influence on the vertical direction. The difference of velocity, diffusion coefficient and disturbing energy between the $x$ - and $y$ dimensions are mild, especially compared to that of the $z$ dimension. The latter also has a higher range between minimum and maximum value at the flow asymmetry extrema.

The directionality of disturbing energy is more pronounced when derived from the Einstein relation compared to the derivation from velocity. This might have to do with the nature of the velocity; the theory of diffusion assumes a purely random process. A bias might affect how this velocity contributes to the observed displacement over time, and this way to the validity of equation 3.2.

A region exist around a flow asymmetry of 0.5 around which the directional dependence is minimum. We are confident that the directional differences between variables can be minimized by proper technical reconstruction of the self-assembly reactor. Altering the number and location of inlet tubes and valves might be one possible option to create a more homogeneous three-dimensional flow-field in which multi-particle self-assembly can be realized.

\subsubsection{Richardson cascade}

The value of the disturbing energy $(k T)$ determined via the two-particle experiments is an order of magnitude lower than that obtained form the diffusion or velocity of a single particle. We speculate that this is because a greater part of the provided energy contributes rather to the motion of single particles than to their close interaction. This speculation is based on the existence of a vortex hierarchy in turbulent flow (Richardson cascade (Richardson, 1926)). The asymmetrical introduction of the turbulent flow causes a macroscopic swirl 
with a diameter close to the tank diameter at the bottom of the cylinder. This swirl moves upward in a screw-like manner. With a strong dominance of inertial forces over viscous forces, the largest eddies are undamped. The turbulence must first break up into smaller vortices by a process of cascading until viscous forces are significant and will dissipate the energy.

Due to this Richardson cascade, there is an energy transfer from the larger vortices to the smaller ones. The energy is not uniformly distributed over the different length scales, but drops off at shorter lengthscales (Hwang and Irons, 2012). So in contrast to thermal fluctuation, the equipartition theorem does not hold for the energy spectrum (turbulence "noise" is not white). When we consider velocity or diffusion, we take into account all vortices. For the two particle experiment, only vortices with length scales in the order of the particle dimensions contribute to their separation. So it is not surprising that the disturbing energy from that experiment is lower.

In addition, the macroscopic swirl may counteract the dominating $z$-direction and strengthen the influence of the $x$ - and $y$-directions on variables. This would explain why the $x$ - and $y$-components are mostly relatively similar.

\subsection{Conclusions}

We created an asymmetric inlet flow into a macroscopic self-assembly reactor. Three of four bottom inlet valves were step-wise closed to the same degree, while the fourth was kept open. This results in a more asymmetric inflow, amplifying the turbulence already created by the reactor geometry. An increase of the turbulence is an increase in the disturbing energy (analogue to thermal energy $k T$ ). We quantified the effect of an increase in turbulence on the motion of spherical centimetre-sized particles in the reactor, characterized by the velocity distribution, diffusion coefficient and disturbing energy.

We characterized the turbulence by tracking the motion of a single sphere. We observed that its most probable velocity as well as the diffusion coefficient obtained from its random walk increases with higher flow asymmetry. From this we conclude that the turbulence in the system is at its lowest value when the water is injected symmetrically (i.e. all four valves are fully open).

Using thermodynamic theory, the velocity distribution and observed diffusion coefficient can be translated to an effective disturbing energy. The estimates for the disturbing energy from the velocity and diffusion are identical within measurement error. In our system, the disturbing energy can be increased from approximately 30 to $200 \mu \mathrm{J}$ (i.e. by a factor around six) by increasing turbulence.

Next to experiment with a single sphere, the disturbing energy was obtained from observation of the interaction between two spheres with embedded magnets. This experiment is more relevant for self-assembly studies. With increased flow asymmetry, the disturbing energy is amplified with approximately a factor 
of three, but the absolute values are an order of magnitude lower than for the single sphere experiment ( 4 up to $12 \mu \mathrm{J}$ compared to 30 to $200 \mu \mathrm{J}$ ).

Since the main flow in the reactor is in the vertical direction, one might expect a directional dependency of the movement of a single sphere. For high turbulence (flow asymmetry higher than 0.5 ), the standard deviation of the velocity distributions in the separate dimensions are similar. For lower turbulence, the standard deviation in the vertical $(z)$ direction is significantly lower than the planar $(x y)$ direction, up to a factor of almost two.

There is a clear directional dependence of the diffusion coefficient. The difference between the horizontal $(x y)$ dimensions are mild. The vertical dimension shows a much stronger dependence of energy on flow asymmetry.

We calculated the directional dependency of the disturbing energy from the velocity distributions as well as the diffusion coefficients. The energy for the $x$ and $y$ dimensions are similar. The energy in the $z$ dimension is more distinct. The derivation from velocity shows similar energy for high flow asymmetry for all three dimensions, but the vertical component is up to a factor two lower for low asymmetry. The derivation from diffusion coefficient shows that the vertical component is much stronger affected by the flow asymmetry.

The thermodynamic conclusions achieved in this investigation have consequences for the study of micro-scale self-assembly. Our results indicate that macro-scale self-assembly can be used as an analogue system in order to study micro-scale self-assembly since we can change turbulence and the disturbing energy in a similar manner as we can change temperature for micro-scale systems. Processes such as crystallization or quenching are likely to be studied analogously at the macroscopic scale provided that and ideally directional homogeneous turbulent flow field can be decreased rapidly in order to achieve quenching. One can take advantage of the possibility of inhomogeneous design which may open up novel venues of investigation. An inhomogeneous turbulent flow field may be interpreted as a temperature gradient. A precisely designed three-dimensional inhomogeneous turbulent flow field may be used for macroscopic mimicking of several processes in for example microscopic mammal or bacterial cells, organelles or organs which for the moment resembles solely a future vision, but simultaneously a most probable achievable target. 


\title{
Chapter 4
}

\section{Dipolar chain and ring formation}

\begin{abstract}
We have studied the interaction of four macroscopic magnetic particles in a turbulent water flow and have investigated which structures are dynamically formed. We have found that the system behaves according to thermodynamic laws by evaluating the frequency of specific structures (lines and rings) forming over time. For this purpose we evaluated images taken from the system from different angles, and evaluated them both manually and by a method of deep learning. This resulted in a relative occurrence of ring structures of $51.1 \%$ and $50.4 \%$ for respectively manual and automated evaluation, versus $36.8 \%$ as predicted by MaxwellBoltzmann statistics. This difference might be explained by underestimating the disturbing energy. We are confident that observation of more complex structures can give insight in how self assembly processes work on microscopic scale.

The work in this chapter is a team effort. My contribution includes composing the theoretical background and writing software that people used to manually analyse the videos. I also developed and implemented the architecture of the neural network, generated the training data, did the network training and data analysis. The reactor was constructed in Twente by Remco Sanders and Léon Woldering and was modified by Per Löthman, who also did data acquisition and arranged manual analysis of the videos. Wesley de Neve, Jasper Zuallaert and Mijung Kim from Ghent University Global Campus have advised about the deep learning architecture.
\end{abstract}

\subsection{Introduction}

Chapter 2 introduced a macroscopic self-assembly reactor as an analogue simulator for microscopic self-assembly processes, and investigated to which extent single and two-sphere systems obey the laws of thermodynamics. Chapter 3 verified the effect of adjusting the turbulence on the behaviour of the par- 
ticles. However, single and two-particle systems are hardly representative for self-assembly.

Therefore, we study the interaction of four spherical magnetic particles suspended in a turbulent water flow. Due to the interaction of magnetic assembling forces and disturbing turbulent forces, the structure is continuously changing shape. Our aim is to determine whether the resulting shapes and their frequency of observation correspond to what we expect based on thermodynamic equations to verify whether our system, also for multi-particle systems, behaves like if it were on microscopic size.

\subsection{Theory}

A chain of magnetic spheres can be characterised by the magnetic potential energy:

$$
E_{\mathrm{m}}=-\frac{1}{2} \sum_{i \neq j} \mathbf{m}_{i} \cdot \mathbf{B}_{j i}
$$

Here, $\mathbf{m}_{i}$ is the magnetic dipole moment of sphere $i$ and $\mathbf{B}_{j i}$ is the magnetic field generated by sphere $j$ on the location of sphere $i$. The field generated by magnetic dipoles can be described by:

$$
\mathbf{B}_{j i}=\frac{\mu_{0}}{4 \pi}\left(\frac{3 \mathbf{r}_{j i}\left(\mathbf{m}_{j} \cdot \mathbf{r}_{j i}\right)}{r^{5}}-\frac{\mathbf{m}_{j}}{r^{3}}\right)
$$

Where $\mu_{0}$ is the permeability constant, $\mathbf{r}_{j i}$ is the distance vector pointing from sphere $j$ to sphere $i$ and $r$ is its norm. Figure 4.1 shows the magnetic energy of a chain as a function of the angle between subsequent spheres. There are energy minima when the chain is a full line (at an angle of 0 ) and when it is a full ring (at an angle of $\pi / 2$ ). The system will tend to move to one of these states, and needs a temporary burst of energy in order to overcome the energy threshold of approximately $35 \mu \mathrm{J}$. Of course the system has more states than the ones depicted in figure 4.1 , but these are not considered in this work.

The occurrence of states of a thermodynamic system can be characterised by Maxwell-Boltzmann statistics. This theory states that the occurrence of a state $n$ is dependent on the energy level of this state $E_{n}$ and the thermal energy of the environment $k T$ :

$$
p_{n} \propto e^{-\frac{E_{n}}{k T}}
$$

If we consider only the states occurring at the energy minima, we can define the relative occurrence. Here we omit all states other than a pure ring and line and just look at the probability of the structure being a line relative to these two states only:

$$
p_{\text {rel,line }}=\frac{p_{\text {line }}}{p_{\text {line }}+p_{\text {ring }}}=\frac{e^{-\frac{E_{\text {line }}}{k T}}}{e^{-\frac{E_{\text {line }}}{k T}}+e^{-\frac{E_{\text {ring }}}{k T}}}
$$




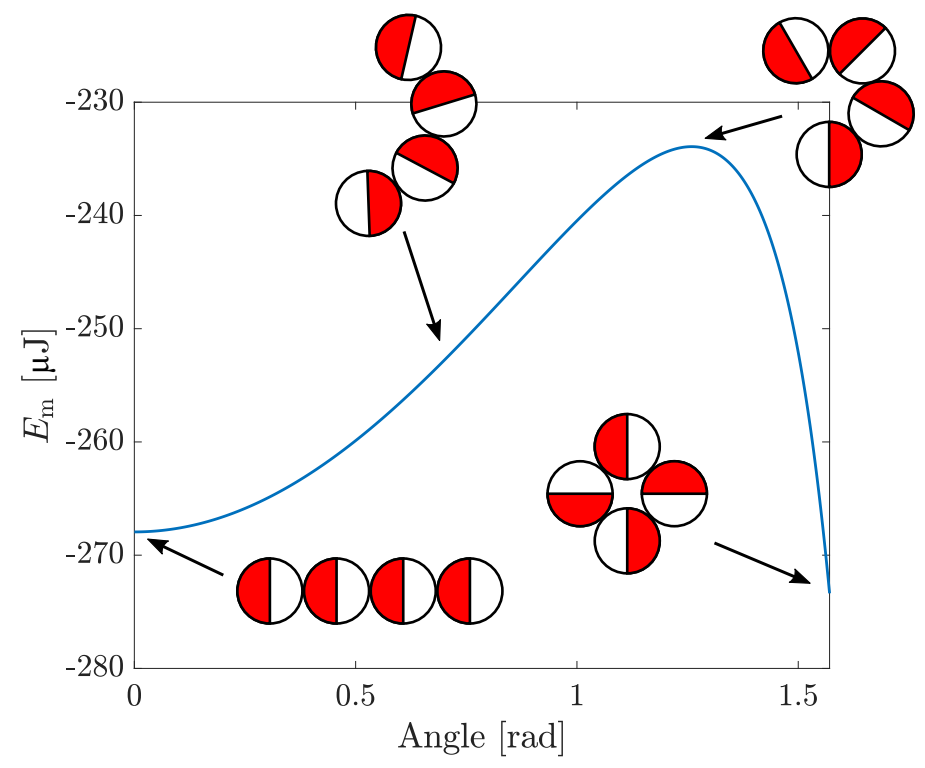

FIGURE 4.1 - The magnetic energy of a chain of magnetic spheres as a function of the angle between subsequent spheres. Energy minima exist where the chain is a straight line (angle is 0 ) and where it is a ring (angle is $\pi / 2$ )

\subsection{Methods}

The experimental setup has been introduced in chapter 2 and has not been modified for this experiment. Four polymeric spheres (diameter $18.5 \mathrm{~mm}$ ) with cylindrical $\mathrm{NdFeB}$ cores (diameter $4 \mathrm{~mm}$, height $4 \mathrm{~mm}$, dipole moment $50.8 \mathrm{~mA} \mathrm{~m}^{2}$ ) are suspended in the flow. With these dimensions, energy calculations using the dipole approximation (equations 4.1 and 4.2 ) are valid with an error of $1.3 \%$. 2200 Frame sets were recorded with intervals of $10 \mathrm{~s}$ to make sure there is no correlation between subsequent frames. This amount of frames yields a sampling error (95\% confidence interval) of $2.1 \%$.

The data was then evaluated and sorted into three different classes; either 'line', 'ring', or 'none', the latter containing every state other than the first two. Figure 4.2 gives a few examples of observed sphere structures along with their correct classification. The classification was performed using two methods; manual evaluation and automated evaluation. 


\subsubsection{Manual evaluation}

This method involved six people observing all of the frame sets one by one and classifying them in a subjective way. Each person has a different opinion about the limits on the classes (how much is the line structure allowed to bend before it stops being a line?), which corresponds to different classification algorithms. The results are then combined by a majority voting scheme, which assigns to each frame set the class which has been chosen most often by the people.

\subsubsection{Automated evaluation}

This method involved automated image classification using deep learning. This requires the definition of a neural network architecture and proper training with a set of training images and corresponding labels. If correctly trained, this network takes as an input a pre-processed image set and outputs the probabilities of the image set being of class 'line', 'ring' and 'none'. This, however, requires the existence of a correctly labelled data set which is absent. As a solution we generated artificial data based on true geometrical shapes; for this purpose we defined 3D structures consisting of four spheres in a specified geometrical configuration. This structure was rotated and translated within limits corresponding to real-life situations, and then projected to 2D using measured camera calibration parameters. The resulting images were adjusted to closely resemble the original data by including a background, applying blurring, particle occlusion and radial gradients on the spheres. Figure 4.4 shows the neural network used. As an input it takes the set of two corresponding images, passes them through a Siamese network consisting of six convolutional layers which share their parameters $W$. After these layers the resulting data is flattened and goes through 3 additional fully connected layers, finally outputting a 3-element vector representing the probabilities of the images being classified as 'line', 'ring' and 'none' respectively. The network was trained on 70000 artificially generated images. The training set is balanced; it contains as much examples of each class. Structures were considered 'lines' if the angle between subsequent particles does not surpass $30^{\circ}$. Structures were considered 'rings' if the angle between subsequent particles is between $85^{\circ}$ and $90^{\circ}$. Structures were considered 'none' in all other cases, including cases with disattached spheres.

\subsection{Results}

The reactor has an estimated value for $k T$ of $6.5 \mu \mathrm{J}$ as determined from the statistical interaction of two spheres (see chapter 2). The magnetic energy of the sphere system in line and ring states are respectively $268 \mu \mathrm{J}$ and $273 \mu \mathrm{J}$ (equation 4.1). Under these conditions, equation 4.4 predicts that 'lines' and 'rings' will appear with a probability of respectively $36.8 \%$ and $63.2 \%$. 

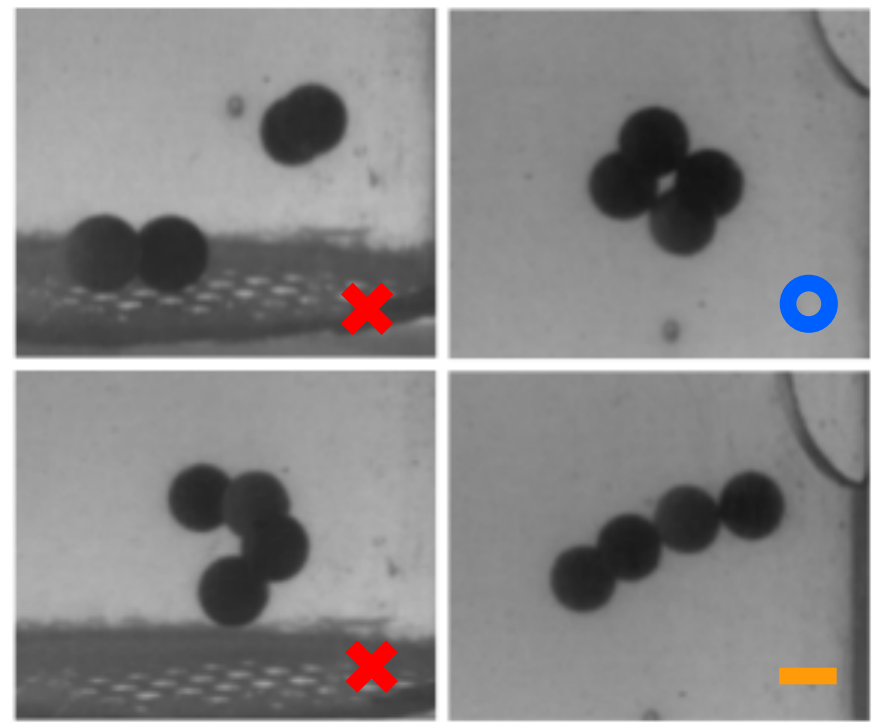

FIGURE 4.2 - Snapshots taken of the four-sphere structure in various states. Disconnected (up left) and curved (down left) structures are classified as 'none,' ring structures (top right) are classified as 'ring', and straight structures (bottom right) are classified as 'line'.

\subsubsection{Human evaluation}

Figure 4.3 shows the relative occurrence of lines and rings. It can be seen that there is a significant disagreement about what classifies as a ring and line; among the different people $p_{\text {rel,ring }}$ ranges from 0.31 to 0.55 . Majority voting yields a conditional probability of $49.0(24) \%$ and $51.0(24) \%$ for ring and line, respectively. The error bars represents the standard deviation resulting from randomly removing a single person from the voting process.

\subsubsection{Automated evaluation}

After having trained the network, it correctly classifies $95.0 \%$ of the training set images. Table 4.1 shows more detailed the performance of the classification. It can be seen that rings and lines are correctly classified with a probability of respectively $95.4 \%$ and $99.7 \%$, and are rarely confused with respect to each other. The 'none' class shows more overlap with the other classes, being misclassified $10 \%$ of the time. 
TABLE 4.1 - Validation of the neural network on artificial data. It shows how often the network correctly classified an image pair based on its true state. Horizontal numbers add up to $100 \%$.

\begin{tabular}{l|lll} 
True state & \multicolumn{3}{|c}{ Neural network classification } \\
& Ring & Line & None \\
\hline Ring & $95.4 \%$ & $0.0 \%$ & $4.6 \%$ \\
Line & $0.0 \%$ & $99.7 \%$ & $0.3 \%$ \\
None & $5.6 \%$ & $4.4 \%$ & $90.0 \%$
\end{tabular}

TABLE 4.2 - Occurrence and relative occurrence of the states 'line', 'ring' and 'none', according to theoretical predictions and manual and automated analysis of experimental data.

\begin{tabular}{c|cc|ccc} 
& \multicolumn{2}{|c}{ Occurrence } & \multicolumn{3}{c}{ Relative occurrence } \\
& Manual & Automated & Theory & Manual & Automated \\
\hline Ring & $41.2 \%$ & $36.8 \%$ & $36.8 \%$ & $51.1 \%$ & $50.4 \%$ \\
Line & $39.3 \%$ & $36.2 \%$ & $63.2 \%$ & $48.9 \%$ & $49.6 \%$ \\
None & $19.5 \%$ & $27.0 \%$ & & &
\end{tabular}

\subsubsection{Overview}

Table 4.2 shows the summarized results, after applying the neural network on the experimental data. In both the manual and automated analyses structures are more often identified as lines and rings than as none. Manual selection more often considers the structure to be a ring or line. If we consider the relative occurrence of lines and rings, we observe that the manual and automated analyses yield very similar results. Considering a sampling error of $2.1 \%$ there is a significant difference between the experimental results and the theoretical prediction.

\subsection{Discussion}

The fact that both evaluation methods provide numbers significantly different to the predicted occurrence is troubling. A possible reason for this phenomenon is that the effective disturbing energy is different than we assumed. In chapter 2 we found that the difference in disturbing energy obtained via single- and two-sphere systems is an order of magnitude. We furthermore reasoned that due to a wavelength-dependent energy spectrum particles and clusters of different size will experience a different amount of disturbing energy. Indeed, as a four-sphere system is significantly larger than a two-sphere system we can expect a higher energy level. An infinite amount of disturbing energy is needed in order to predict a relative occurrence of 50/50\% for lines and rings. Starting from $27 \mu \mathrm{J}$ the predicted odds are closer to the observed occurrences (45/55\%). This value is still well within the range of values of $k T$ found for two-sphere (approximately $6.5 \mu \mathrm{J}$ ) and single-sphere (approximately $80 \mu \mathrm{J}$ ) systems. We do 


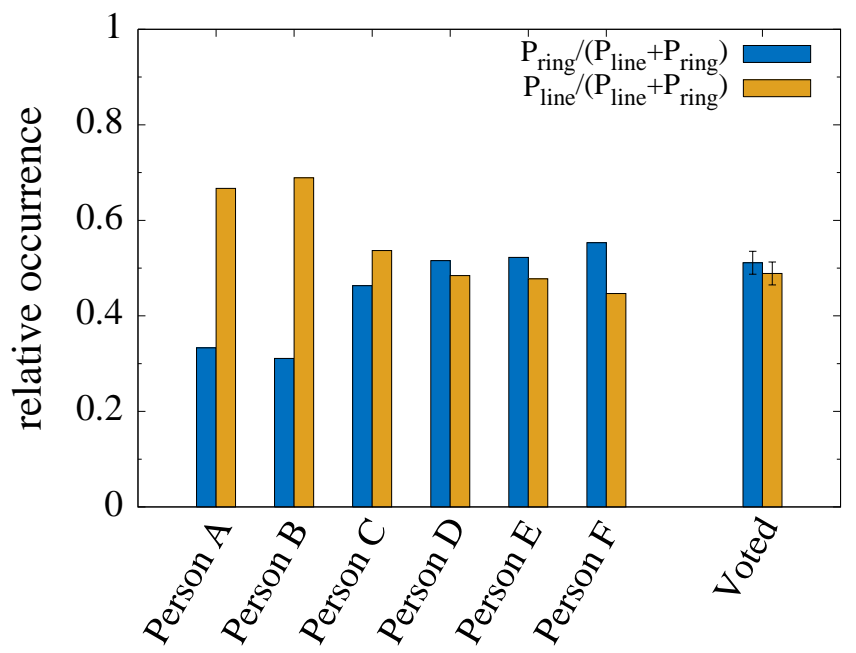

FIGURE 4.3 - The relative occurrence of ring and line structures among 2000 frames, as analysed by several people. There is a significant disagreement among different people. Majority voting provides a more accurate result.

not rule out the influence of other effects, such as hydrodynamic forces, manufacturing tolerances and errors due to the dipole approximation, especially considering the small difference between the energy levels.

Apart from the mismatch between theoretical and experimental results the methods of analysis need to be addressed. First of all, subjective evaluation by people is prone to bias and is not stable over time. Even though the influence of these effects is reduced by majority voting, this method can still result in a bias due to the fact that selection is subjective; there are no strict geometric definitions of what is a line and ring. Still, human observation is much more time efficient for the used amount of data than the time consuming process of generating artificial data and training neural networks.

Strict geometric definitions are present in the case of deep learning. The training set is large enough to cover most possible states of the system. Still, there is no $100 \%$ success rate in classifying even the artificial images. One reason is that in order to cover more possible states, a random factor is involved in generating the sphere structures. This random factor can result in overlapping classes, resulting in errors. A second factor is that the network might not be optimal. The architectures are chosen by a method of trial-and-error such that optimization is hard and we settle for a non-optimal network. A more serious issue is that the network is trained on artificial data and that we use it to classify experimental data, which is slightly different in appearance. This expectedly res- 


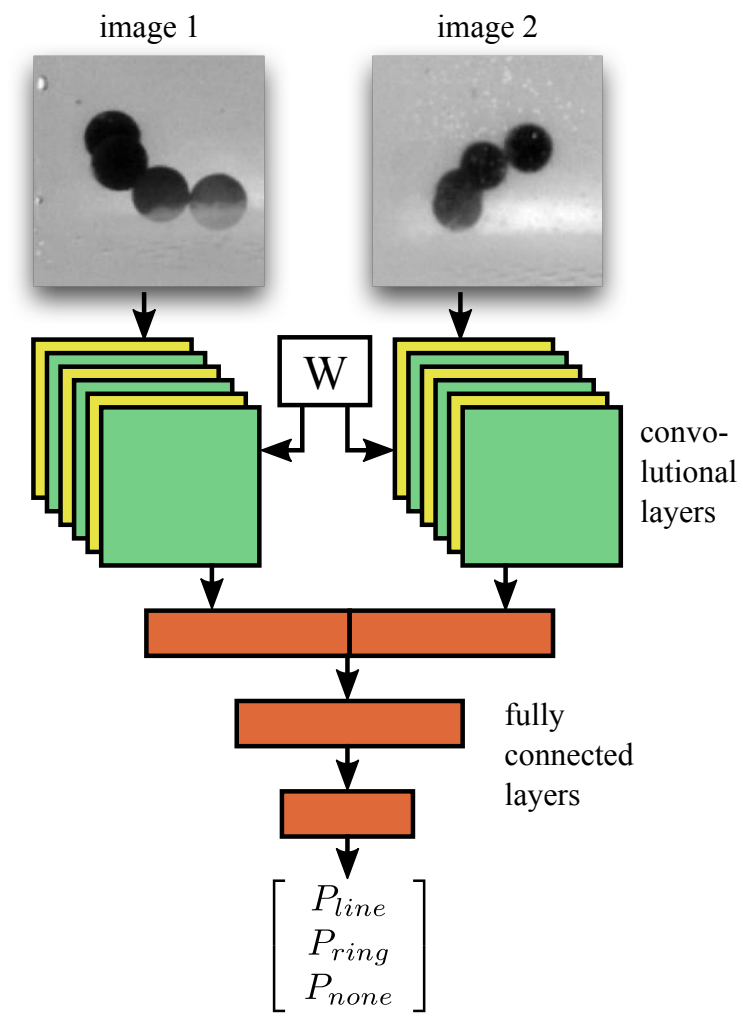

FIGURE 4.4 - The architecture of the neural network used to analyse the images. It consists out of six Siamese convolutional layers, which share the same weights between them, followed by three fully connected layers.

ults in a reduction in classification accuracy. We are unable to verify how well the system performs on experimental data because there is no ground truth for the labels. The only available labels are given by human analysis, which are not guaranteed to be correct.

As a last thing, we have only considered the extreme states of 'line' and 'ring'. However, these perfect states occur only rarely as most often there is a small defect in the observed shapes like a slight bend. By accepting only the perfect shapes, we do not have a large enough set to make statistical significant conclusions. Furthermore, distinguishing between the ideal and non-ideal shapes is a very challenging assignment due to the structure being three-dimensional, which makes good observation hard. Therefore, many non-ideal shapes have been accepted as lines and rings, even though they contain different energy levels than those ideal shapes. 


\subsection{Conclusions}

In this work we evaluated the structures formed by four interacting magnetic spheres in a turbulent water flow. The main goal was to evaluate the frequency of line- and ring-shaped structures, and compare this with the values as predicted by thermodynamic theory. For this purpose we evaluated 2200 snapshots of the structures manually as well as by deep learning.

Manual analysis based on 6 subjects gives a relative occurrence of $51.1 \%$ and $48.9 \%$ for ring- and line structures, respectively. Deep learning predicts a relative occurrence of $50.4 \%$ and $49.6 \%$, respectively. This deviates from respective values of $36.8 \%$ and $63.2 \%$ as predicted by Maxwell-Boltzmann statistics, an error more significant than the sampling error of $2.1 \%$. This indicates we might have underestimated the equivalent thermal energy of the turbulent flow.

These results suggest that more careful characterisation of the disturbing energy is required. We still trust that further studies of systems consisting of more particles and different geometries will give valuable insights in how self assembly processes work on microscopic scale, which can be exploited for optimization of micro-fabrication processes. 


\title{
Chapter 5
}

\section{Three-dimensional cluster growth of magnetic particles}

\begin{abstract}
We have studied the structures formed by three-dimensional, macroscopic self-assembly and found that the geometry of particles is highly decisive for the type of structures are being formed. Centimetre-sized particles of various geometries (ellipsoids, cylinders, cubes) and aspect ratios with embedded magnets were suspended in an upward flow gradient, forming structures by an interplay between magnetic assemblingand turbulent disturbing forces. Ellipsoidal particles formed stacked ringlike and opaque structures, while cylindrical and cubic particles formed more regular structures with straight angles. For the latter, the aspect ratio gives us control over the formed structures, being one-, two- and threedimensional in nature for respectively low, high and medium aspect ratio. The study of self-assembly dynamics on macroscopic scale can give valuable information about design decisions for microscopic self-assembly, while offering more simplified particle design and observation techniques.

The work in this chapter is a team effort. My contribution includes composing the theoretical background, designing and constructing the particles and performing the experiments. The reactor was constructed in Twente by Remco Sanders and Léon Woldering and was modified by Per Löthman, who also designed the cone inset.
\end{abstract}

\subsection{Introduction}

The previous chapters dealt with characterisation of the macroscopic self-assembly reactor and assessing to which extent the behaviour of particles obey the laws of thermodynamics.

The study of macroscopic magnetic self-assembling systems is not new. Several groups have investigated the influence of particle- and reactor design, as well as environmental conditions on the formed structures (figure 5.1). 
Stambaugh et al. (2003) investigated a vertically-shaken mono layer of centimetre-sized magnetic spheres. The observed patterns were highly dependent on the particle density, reactor shape, magnet shape and initial particle distribution. Miyashita et al. (2009) investigated the influence of geometry on structure formation of floating magnetic particles while being shaken. They quantified the aggregation characteristics and showed that rounded particles assemble faster than squared ones and that the early stages of the aggregation pattern are crucially influential to the rest of the process. Hacohen et al. (2015) investigated programmed assembly of macroscopic magnetic bricks by patterning the sides of tetrahedrons, which assemble after agitation. These works are primarily two-dimensional in nature, however, and are not representative for true three-dimensional systems. Ilievski et al. (2011b) investigated buoyancyneutral magnetic particles in a three-dimensional turbulence-driven assembly process, forming one-dimensional structures. They found that statistical mechanics predict well how the strength of turbulence and the magnetic attraction forces influence the obtained chain length.

We expand upon this work by moving to fully three-dimensional shapes and investigating the influence of geometry of magnetic particles and their amount on the resulting structures. Patterns with lowest energy will have the highest probability of occurring. In a buoyancy-neutral system, using the magnetic dipole approximation, magnetic potential energy is dominant:

$$
E_{\mathrm{m}}=-1 / 2 \sum_{i \neq j} \mathbf{m}_{i} \cdot \mathbf{B}_{j i},
$$

where $\mathbf{m}_{i}$ is the magnetic moment of particle $i$ and $\mathbf{B}_{j i}$ is the field generated by particle $j$ on the location of particle $i$. The probability that particles are connected axial ( $p_{\mathrm{ax}}$, with energy level $E_{\mathrm{ax}}$ ) or diametrically ( $p_{\text {diam }}$, with energy level $\left.E_{\text {diam }}\right)$ are related to their relative energy level:

$$
\frac{p_{\mathrm{ax}}}{p_{\mathrm{diam}}} \propto e^{\frac{E_{\mathrm{ax}}-E_{\mathrm{diam}}}{k T}}
$$

The aspect ratio allows for tuning of the connection energy and thereby particle interaction; wide particles $\left(E_{\mathrm{ax}}-E_{\text {diam }}<0\right)$ will be biased towards endto-end connections such as chain-like structures, while long particles ( $E_{\mathrm{ax}}-$ $E_{\text {diam }}>0$ ) are biased to side-to-side connections such as planar structures. For $E_{\mathrm{ax}}-E_{\mathrm{diam}}=0$ both types of connections will be common, able to form regular, three-dimensional structures.

\subsection{Results}

\subsubsection{Reactor}

Figure 5.2 shows a schematic of the system previously introduced and characterised in (Hageman et al., 2018). Particles are submerged in a cylindrical reactor 


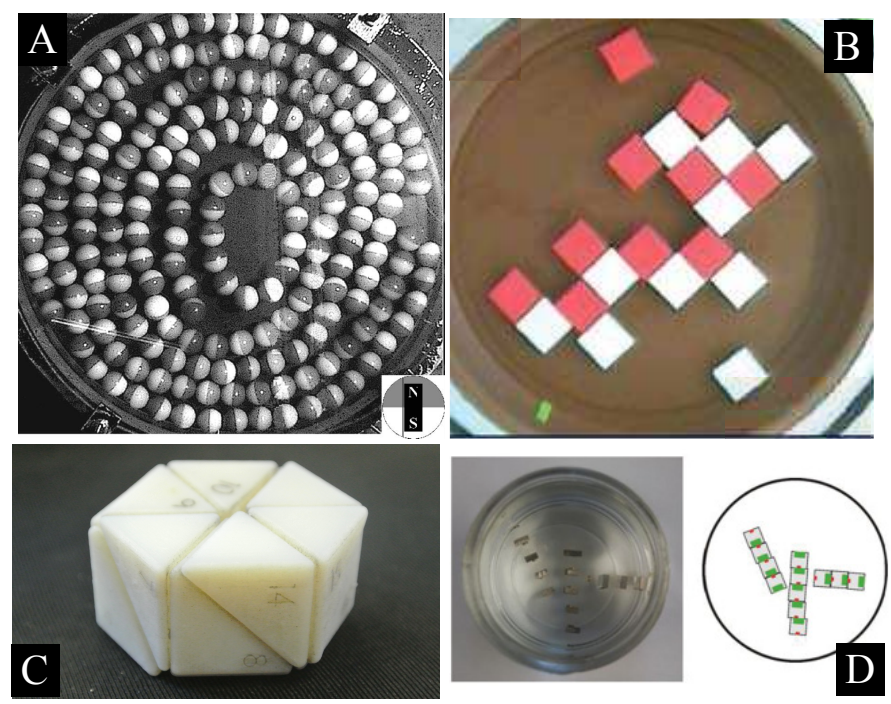

FIGURE 5.1 - A: Centimetre-sized magnetic particles shaken vertically form patterns inside a two-dimensional plane (Stambaugh et al., 2003). B: Centimetre-sized floating magnetic cubes self-assemble in patterns when shaken (Miyashita et al., 2009). C: Uniquely patterned bricks assemble into a product through shaking (Hacohen et al., 2015). D: Turbulence driven three-dimensional self-assembly creates line structures out of centimetre-sized magnetic cubes (Ilievski et al., 2011b).

tube with a turbulent upward-directed water flow, both for counteracting gravity and a as a source of disturbing energy. A cone-shaped inset creates a flow gradient meant to center particles in the middle and prevent interaction with the top- and bottom nets to contain the particles.

\subsubsection{Particles}

3D printed, centimetre-sized particles have a cylindrical, axially-magnetized $\mathrm{NdFeB}$ core, and is color coded based on polarisation. They are designed such that $E_{\mathrm{ax}}-E_{\mathrm{diam}} \in\{-40,0,40\} \mu \mathrm{J}$, in order of increasing aspect ratio (AR), while ensuring that their minimum connection energy stays $-80 \mu \mathrm{J}$. Particles are either ellipsoidal, cylindrical, or cubical, ordered in increasing extent to which the particle poses geometrical restrictions on how they can connect.

\subsubsection{Experiments}

Particles in various amounts $(8,12,16)$ were inserted into the reactor with appropriate flow speed settings to create neutral buoyancy. The level of turbulence 
in all cases was high enough to overcome the magnetic attraction, allowing observation of particle interaction while the system explores the energy landscape. Figure 5.3 shows typical structures formed by 8 particles of various geometries.

A clear dependence of structures based on geometry can be observed. Arguably the most important factor is aspect ratio, followed by geometry (ellipsoids vs other structures). Ellipsoidal particles form ring-based structures of which the amount of particles per ring is dependent on the AR. We observe most frequently a 3-, 4- and 8-ring as main building block of the structures. The latter is a result of solely axial connections, and will increase with the amount of particles in the reactor, and is mainly limited by the reactor size. The medium AR ellipsoids are in a very stable configuration and rarely escape from their stacked 4-ring structure.

Ellipsoidal structures are quite distinct from the structures formed by cylindrical and cubical particles, the latter ones being quite similar. These particles form mainly straight angles between one another and are more rigid than the ellipsoidal connections. Although high AR particles connect diametrically, they can also connect diametrically under a $90^{\circ}$ angle. This allows them to escape a purely two-dimensional configuration. Medium AR particles connect both axially and diametrically, forming true regular three-dimensional structures. Cylindrical particles more easily evolve from two-dimensional to threedimensional structures, as there are less geometrical constraints on stable connections. Low AR particles rarely connect different from axially, forming rigid one-dimensional structures rather than the flexible chains created by ellipsoidal particles. Reactor dimensions, however, pose a clear restriction on the length of the structure.

Figure 5.4 shows typical structures formed by 8, 12 and 16 medium AR particles in the reactor. Elliptical particles form truly different shapes. The stacked four-ring who dominated the 8-particle experiment is broken up after adding more particles. Now also stacked three-ring structures and less defined, amorphous structures can be observed. The increased amount of particles this way acts as a catalyst.

There is no fundamental change in the structures formed by cylindrical and cubical particles. They are still connected diametrically and axially, but form more complex structures. This is to be expected due to the increase in the amount of particles and consequently the amount of states.

\subsection{Conclusions}

We investigated the influence of the aspect ratio and geometry of magnetic particles on resulting structures during a three-dimensional self-assembly process.

The geometry of the particles determine the regularity of structures. Ellipses tend to form stacked ring structures as well less defined, amorphous structures. In contrast, cylindrical and cubical particles form more rigid structures characterised by straight angles. The aspect ratio (AR) determines the magnetic 


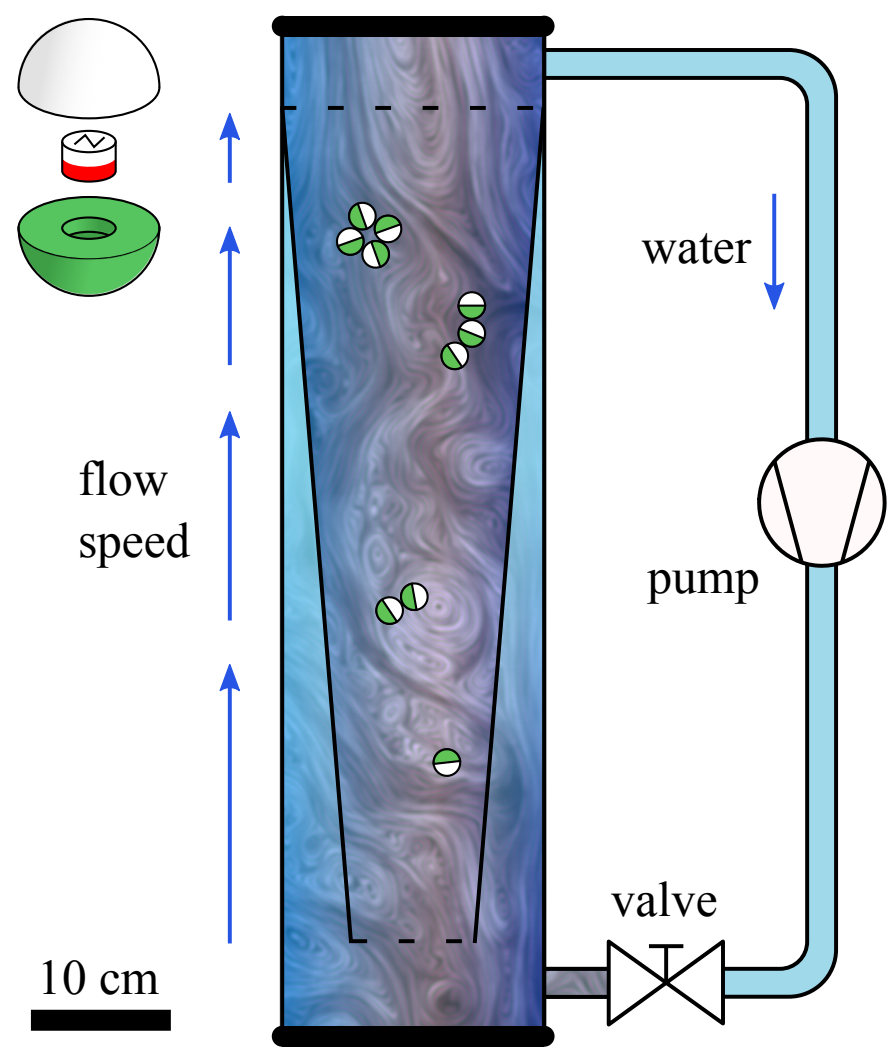

FIGURE 5.2 - The self-assembly reactor pumps water upwards to create neutral buoyancy for the particles and to introduce a turbulent flow. A cone-shaped inset creates a flow gradient to keep the particles on specified height and minimizes their interaction with the meshes on either side of the reactor. Particles are 3D-printed and contain a cylindrical NdFeB magnet (top left).

potential energy of connected particles. Low AR particles form primarily onedimensional structures. Medium AR particles allow both axial and diametrical connections and therefore support fully three-dimensional structures. High AR particles prefer diametrical connections, forming two-dimensional structures, but also allow diametrical connections under an angle of $90^{\circ}$, offering an escape to the third dimension.

Increasing the amount of particles generally increases the complexity of structures due to the increased amount of states. It additionally can act as a catalyst to break up generally stable structures. 


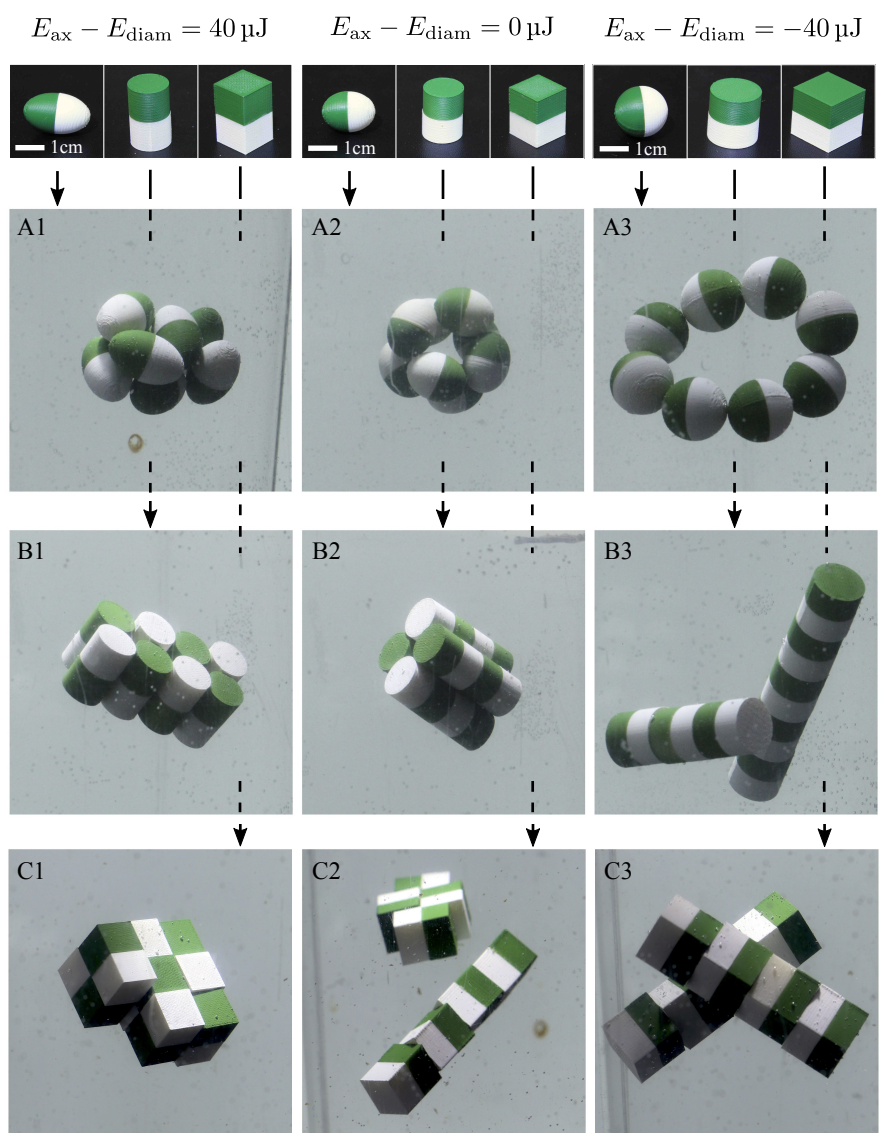

FIGURE 5.3 - The aspect ratio (AR) and particle shape heavily influence the resulting clusters. Ellipsoidal particles $\left(A^{*}\right)$ tend to form flexible (stacked) ring-based structures while cylindrical $\left(B^{*}\right)$ and cubical particles $\left(C^{*}\right)$ primarily connect via straight angles, resulting in more rigid structures. The AR of ellipsoidal particles determine the most common observed amount of particles per ring, which is 3, 4 and 8 for respectively high (A1), medium (A2) and low AR particles (A3). High AR cylinders and cuboids connect primarily diametrically (B1, C1), but can also connect under a $90^{\circ}$ angle, allowing them to escape a fully two-dimensional configuration. Medium AR cylinders and cuboids connect both axially and diametrically and can form true three-dimensional structures (B2, C2). Low AR cylinders and cuboids form primarily one-dimensional structures (B3, C3). 


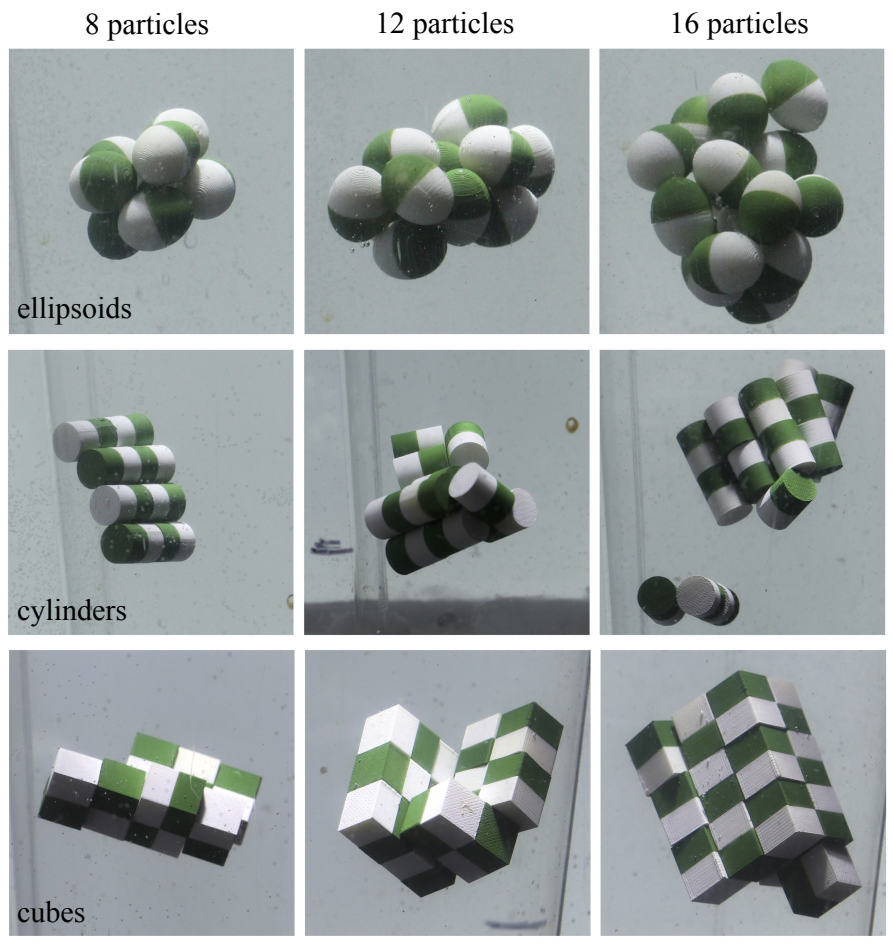

FIGURE 5.4 - Systems of 8 (left), 12 (middle) and 16 (right) particles of elliptical (top), cylindrical (middle) and cubical (bottom) geometry. The resulting structures are more complex due to the increased amount of particles. The stable stacked four-ring structure which dominated the 8-ellipsoid experiment is broken up after adding more particles, acting as a catalyst. Now also stacked three-ring structures are observed, as well as less defined, amorphous structures.

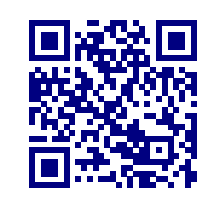




\title{
Chapter 6
}

\section{Kinetics of 2D contrained orbitally-shaken particles}

\begin{abstract}
We present an experimental study of the kinetics of orbitally-shaken macroscopic particles confined to a two-dimensional bounded domain. Discounting the forcing action of the external periodic actuation, the particles show translational velocities and diffusivity consistent with a confined random walk model. Such experimental system may therefore represent a suitable macroscopic analogue to investigate aspects of molecular dynamics and self-assembly.

The work in this chapter is a team effort. My contribution includes composing the theoretical background and performing the experiments. I wrote the software for particle detection, color-based identification, orientation estimation and tracking and performed the statistical analysis. The experiment was designed by Dhananjay Ipparthi and Massimo Mastrangeli, who additionally constructed the setup and the particles and were involved in every step along the way.
\end{abstract}

\subsection{Introduction}

Concepts pertaining to self-assembly can explain a variety of natural phenomena occurring across different scales, from molecular to macroscopic (Klug, 1983; Whitesides and Boncheva, 2002; Whitesides and Grzybowski, 2002). An inherent difficulty in studying the self-assembly of molecular systems is posed by the very size of the self-assembling agents, here generically defined as particles, and by the short duration of their interactions. As an alternative to fast spectroscopic techniques (Calegari et al., 2014), analog macroscopic models of self-assembling systems can provide magnified if approximate representations of the interactions between particles and of their time scales amenable to easier investigations (Hageman et al., 2015). Analog macroscopic models have 
proven useful to study at least two aspects of self-assembling systems: particles' kinetics (Grzybowski et al., 2002) and population/concentration dynamics (Hosokawa et al., 1994; Miyashita et al., 2011). Time evolution of particle populations has been theoretically studied using difference equations (Gillespie, 2007; Hosokawa et al., 1994; Miyashita et al., 2011). The simulated results of these works show qualitative correspondence with experimental data obtained from systems of macroscopic particles. Among available options (Kudrolli, 2004; Kumar et al., 2015; Ojha et al., 2004), orbital shaking is a useful agitation method for macroscopic setups to study the dynamics and interactions of granular matter (Bhalla et al., 2014; Cademartiri et al., 2012; Grünwald et al., 2016; Hacohen et al., 2015; Tricard et al., 2013, 2015). The statistics of the motion that orbital shaking imparts to solid, in-plane bound particles was however not characterised to date.

In this chapter we assess the motion of macroscopic, orbitally-shaken particles confined to a two-dimensional (2D) bounded space, and whether it may approximate the motion of molecules in a highly diluted 2D solution. The statistics of diffusing particles (Gillespie and Seitaridou, 2012) is described by random walk and Brownian motion models (Einstein, 1905; Langevin, 1908; Smoluchowski, 1916). In our analogy with the macroscopic realm, we adopt the latter as guiding frameworks to interpret our experimental data, though the mechanism underlying our particles' motion is admittedly different.

\subsection{Experimental setup}

Our experimental setup was composed of a 2D circular reactor confining 3Dprinted particles, an orbital shaker (orbit diameter $d_{\text {orb }}=2.5(1) \mathrm{cm}$ ) imparting motion to the particles through the reactor, and an overhead camera for optical tracking (figure 6.1). The particles were $7 \mathrm{~mm}$ thick, geometrically equal sectors of a circle with radius of $25 \mathrm{~mm}$ and spanning angle of $45^{\circ}$. The particles' homogeneous colour and shape were chosen to facilitate the tracking of their positions and angular orientations, respectively. The circular reactor with inner diameter of $250 \mathrm{~mm}$ had rough interior surface, as we found surface roughness to reduce friction and improve the mobility of the particles. The camera was made solidal to the non-inertial frame of the shaker through a mechanical arm to avoid the need for shaker motion subtraction prior to image analysis.

The experiments were carried out under two conditions, whereby the motion of 1 and 3 particles were respectively tracked. Each experiment was performed by placing the particle(s) into the closed reactor, starting the video frame capture at $20 \mathrm{fps}$, and then running the shaker at a frequency $f=5.00$ (2) Hz. Each experiment was run for $8 \mathrm{~min} 20 \mathrm{~s}$ to capture 10000 frames. Image processing of each acquired frame involved background subtraction, low-pass filtering and linear discriminant analysis to isolate the colour blobs corresponding to the particles based on their RGB value. Morphological operations were used to clean the blobs of remaining artefacts. Within the shaker frame, the 


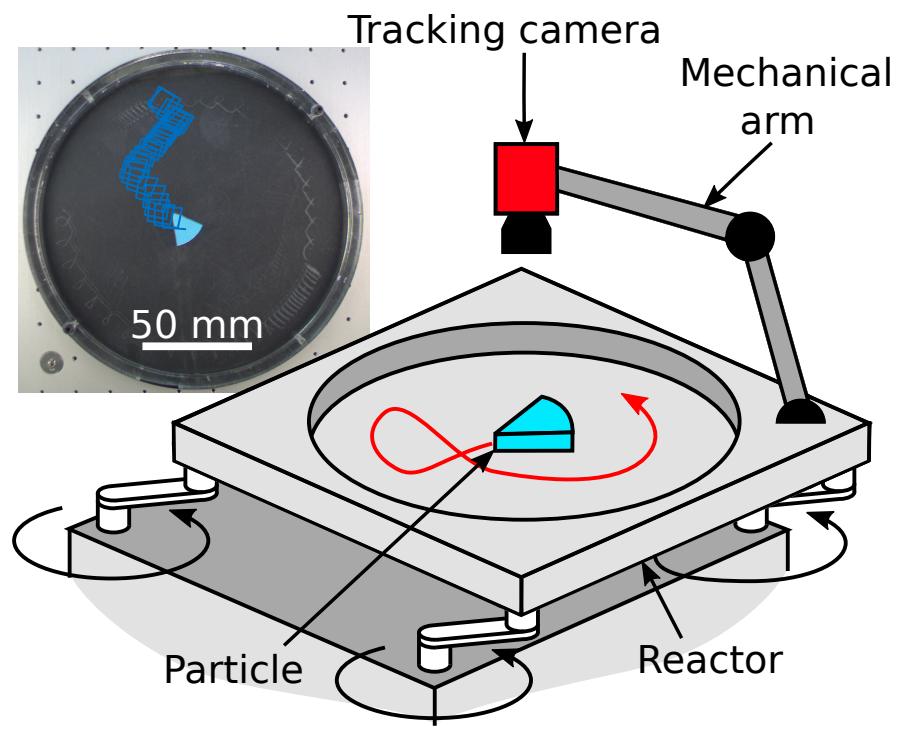

FIGURE 6.1 - Schematic of the experimental setup used in this study. A single particle and its trajectory, tracked through the overhanging camera solidal with the circular $2 D$ reactor, are shown in the inset (see figure 6.2b-c for the 3 particles case).

position $\mathbf{x}$ of each particle was assumed to be the centre of mass of the corresponding blob. The orientation $\theta$ of each particle was obtained by fitting lines onto the straight edges of the particles and evaluating the subtended angle in the shaker frame of reference.

\subsection{Results}

We analysed three aspects of the particle kinetics: (1) velocity distribution, (2) translational diffusion and (3) rotational diffusion. Diffusion is the motion of particles caused by thermal energy (Cooksy, 2014). In the random walk model of Brownian motion, the translational velocities of particles with $k$ translational degrees of freedom are $\chi_{k}$-distributed (Berg, 1993). The velocities of particles moving by Brownian motion in three dimensions follow a $\chi_{3}$ distribution, i.e., a Maxwell-Boltzmann distribution (Berg, 1993). Since in our case we allow the particles to move in two dimensions $(k=2)$, we might expect the $2 \mathrm{D}$ translational velocities and their $1 \mathrm{D}$ projections to respectively follow a $\chi_{2}$ distribution, i.e., a Rayleigh distribution

$$
f(\nu, \sigma)=\frac{v}{\sigma^{2}} e^{-\nu^{2} /\left(2 \sigma^{2}\right)}
$$


with velocity $v$ and velocity mode $\sigma$, and a $\chi_{1}$ distribution, i.e., a Gaussian, if a diffusion analogy holds for our system.

In the kinetic analysis, we first consider the constant orbital component of the particle motion, expressed in the form of repetitive, short-range circular trajectories superposed to the linear, long-range displacements of the particles (figure6.1-inset and figure 6.2b). Such orbital motion, due to the global actuation forced by the shaker, equally and synchronously affects all particles. Therefore, while causing the motion of the particles, the orbital motion does not primarily contribute to particle interactions in a sparse particle system. Particle interactions are mainly due to relative motion differences, as induced by e.g. mutual collisions and boundary effects. We discounted for the constant orbital component in the analysis of particle trajectories to focus on relative particle motion. The external actuation frequency was consequently omitted from the tracking data by designing a band-stop filter in the frequency domain, to mask the spectrum of the Fast Fourier Transform of the 1D projections of particle trajectories (figure 6.2a), and then inverse-transforming the filtered trajectories back to the time-space domain (figure 6.2c). The filter, with center frequency of $5 \mathrm{~Hz}$ and width of $2 \mathrm{~Hz}$ (figure 6.2a), only suppressed the actuation frequency, and did not alter the phase behaviour of the particle motion. After filtering, the motion of the particles is mainly characterised by low frequencies, whose normalised amplitude decayed by a factor 100 at $1 \mathrm{~Hz}$.

\subsubsection{Velocity distribution}

Figure 6.3-left shows a typical unfiltered 2D velocity distribution of a single orbitally-shaken particle tracked in the reactor, characterised by a mean of $33.42(3) \mathrm{cm} \mathrm{s}^{-1}$ and a standard deviation of $2.9 \mathrm{~cm} \mathrm{~s}^{-1}$. The mean particle velocity is expectedly close to the maximal orbital speed afforded by the shaker, i.e., $v_{\text {orb }} \approx f \pi d_{\text {orb }}\left(=39(2) \mathrm{cm} \mathrm{s}^{-1}\right)$

The filtered 2D particle velocity distribution appears to be Rayleigh-like, as shown by the fitting in figure 6.3-right. A fitting routine for the Rayleigh distribution (Eq. 6.1) was used which minimizes the maximum distance $D_{\max }$ between the cumulative distribution (CDF) of the velocity measurements and the cumulative Rayleigh distribution, yielding the fitting parameter $\sigma$ and its uncertainty $\epsilon_{\sigma}$. A Kolmogorov-Smirnoff (K-S) test was used to quantify the goodness of fit (GOF) and obtain a significance level $Q$ to disproof the null hypothesis that the two distributions are the same (Press et al., 1992). The results of the fit and the K-S test are presented in Table 6.1, and the normalised CDF of the experimental velocities and the fitted Rayleigh function are presented in figure 6.4. Despite the apparent quality of the fit represented in figure 6.3-right, using a Q-value of $5 \%$ as the limit for rejecting the hypothesis the GOF test suggests that the velocities are actually not Rayleigh distributed. The large sample size (9600) makes the analysis very sensitive to subtle deviations from a true Brownian motion, possibly arising from local variations in roughness and flatness of the reactor surface, and particularly from the presence of boundaries. Closer ex- 


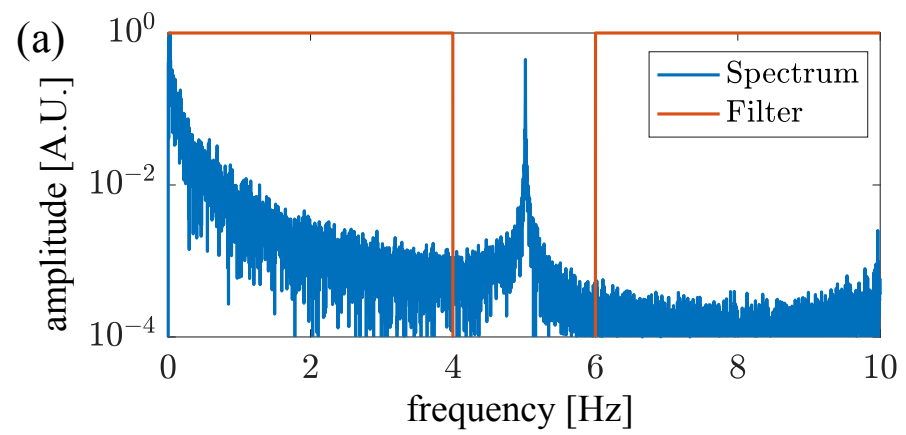

(b)

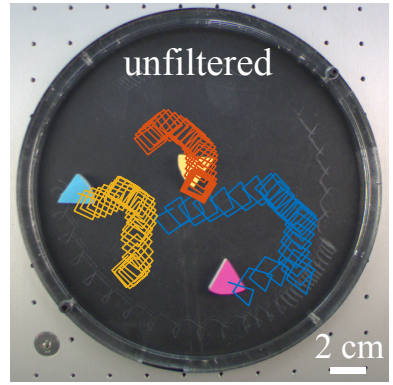

(c)

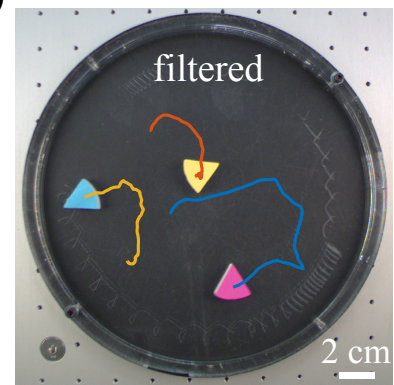

FIGURE 6.2 - (a) Typical spectrum of the $\mathrm{x}$-coordinate of a particle, normalised to a maximum amplitude of 1 , along with the band-stop filter designed to exclude the actuation frequency of the orbital shaker. (b) Unfiltered and (c) filtered trajectories of 3 particles recorded over a period of $5 \mathrm{~s}$.

amination suggests that the observed velocities in the tail of the experimental distribution (figure 6.3-right) are consistently higher than in the fitted Rayleigh distribution, as also evident in figure 6.4. figure 6.5 additionally evidences that higher velocities were observed along the edges of the reactor. This supports that upon collisions the edges impulsively transmitted kinetic energy to the components and raised their velocities. Notably, by omitting particle velocities above $10 \mathrm{~cm} \mathrm{~s}^{-1}$ from the distribution a significantly improved GOF was obtained, as also presented in Table 6.1.

As mentioned earlier, we might furthermore expect the distributions of particle velocity projected over orthogonal, one-dimensional axes to be Gaussian (i.e., $\chi_{1}$ ) distributions; and, for a given particle system, the velocity distribution parameter $\sigma$ to be similar. The 1D projected velocity distributions for a single particle in the reactor are shown in figure 6.6, together with the Gaussian fit obtained using the same fitting procedure earlier described. The results of the fit and K-S test are presented in Table 6.1. The fitting parameter $\sigma$ for the $1 \mathrm{D}_{x}$ and 2D velocity distributions are similar, while this is not the case for the $1 \mathrm{D}_{y}$ distribution. The GOF test suggests that the $y$-velocity is not Gaussian dis- 
TABLE 6.1 - Fitting of the velocity distributions. Values for velocity distribution parameter $\sigma$ with uncertainty $\epsilon_{\sigma}$, and for significance level $Q$ corresponding to the error measure $D_{\max }$ obtained from the $K-S$ test are reported when using either all velocity data (top) or excluding velocity values over $10 \mathrm{~cm} \mathrm{~s}^{-1}$ to omit the effect of reactor boundaries (bottom).

\begin{tabular}{cccccc} 
Samples & Dimensionality & $\begin{array}{c}\sigma \\
{\left[\mathrm{cm} \mathrm{s}^{-1}\right]}\end{array}$ & $\begin{array}{c}\epsilon_{\sigma} \\
{\left[\mathrm{cm} \mathrm{s}^{-1}\right]}\end{array}$ & $\begin{array}{c}D_{\max } \\
{[\%]}\end{array}$ & $\mathrm{Q}$ \\
\hline \hline All & $2 \mathrm{D}$ & 2.45 & 0.02 & 2.6 & $3.7 \mathrm{E}-6$ \\
& $1 \mathrm{D}_{x}$ & 2.48 & 0.03 & 1.3 & $8.6 \mathrm{E}-2$ \\
& $1 \mathrm{D}_{y}$ & 2.34 & 0.04 & 2.1 & $3.8 \mathrm{E}-4$ \\
\hline$\nu \leq 10 \mathrm{~cm} \mathrm{~s}^{-1}$ & $2 \mathrm{D}$ & 2.39 & 0.02 & 1.7 & $6.2 \mathrm{E}-3$ \\
& $1 \mathrm{D}_{x}$ & 2.40 & 0.03 & 1.2 & $1.5 \mathrm{E}-1$ \\
& $1 \mathrm{D}_{y}$ & 2.32 & 0.04 & 2.4 & $3.0 \mathrm{E}-5$
\end{tabular}
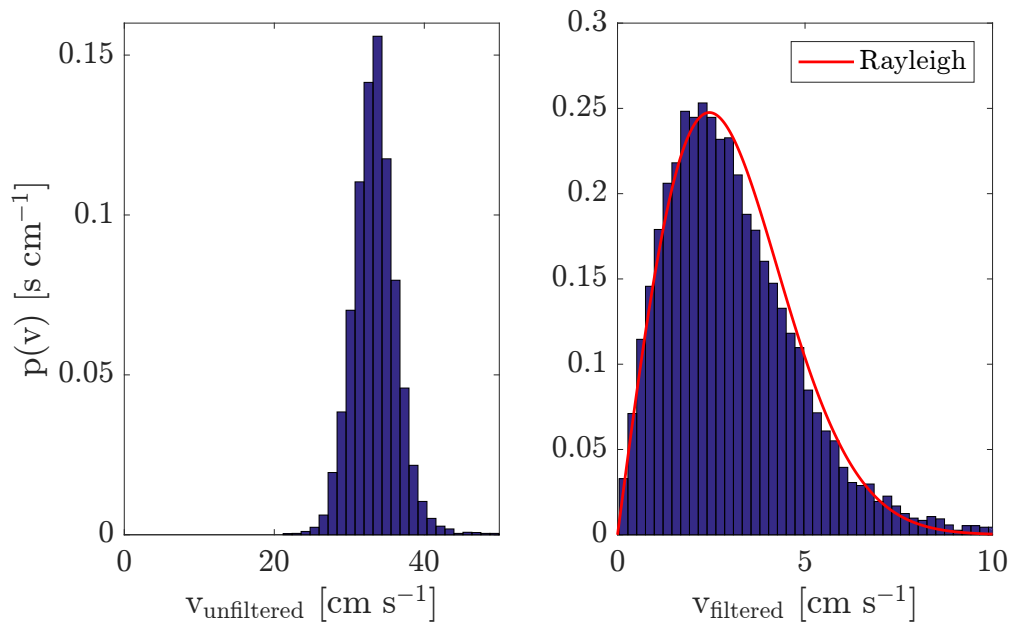

FIGURE 6.3 - Typical 2D velocity distribution for an unfiltered (left) and filtered (right) trajectory of a single particle tracked in the reactor.

tributed. Omitting the velocities in excess of $10 \mathrm{~cm} \mathrm{~s}^{-1}$ observed at the edges, the GOF for $x$-velocity improves, but it deteriorates for $y$-velocity. The velocity distributions and corresponding $\chi_{k}$ fits for the 3-particle experiment, presented in Appendix A, show a more pronounced deviation from an ideal Brownian behavior, possibly reflecting the effect of inter-particle collisions. 


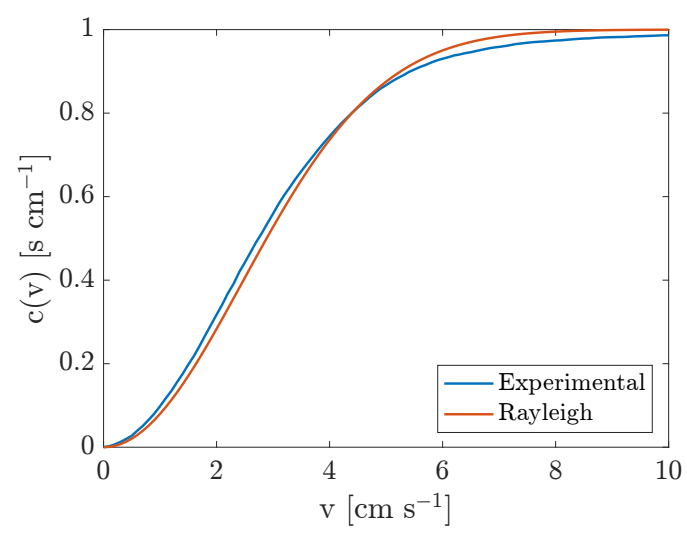

FIGURE 6.4 - Normalised cumulative distribution of the measured and filtered $2 D$ velocity of a single particle (see figure 6.3) and the fitted cumulative Rayleigh distribution.
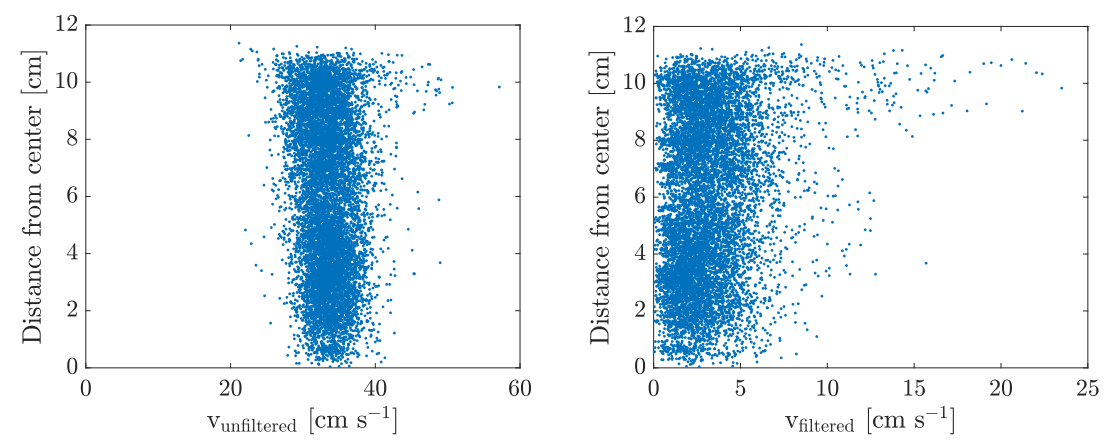

FIGURE 6.5 - Scatter plots of (left) unfiltered and (right) filtered particle velocity versus particle distance from the centre of the reactor.

\subsubsection{Translational diffusion}

To study the diffusion of a particle in the reactor, we consider its complete trajectory, divide it into equal-length sub-trajectories and compute averages of the square displacement, defined as the Euclidean distance from the starting point of the trajectory. For a two-dimensional system, we might expect the following relation:

$$
\left\langle X^{2}\right\rangle=4 D t
$$

where $\left\langle X^{2}\right\rangle$ is the mean square displacement, $D$ the diffusion coefficient and $t$ the time (Einstein, 1905; Perrin, 1909; Smoluchowski, 1916). Choosing more 

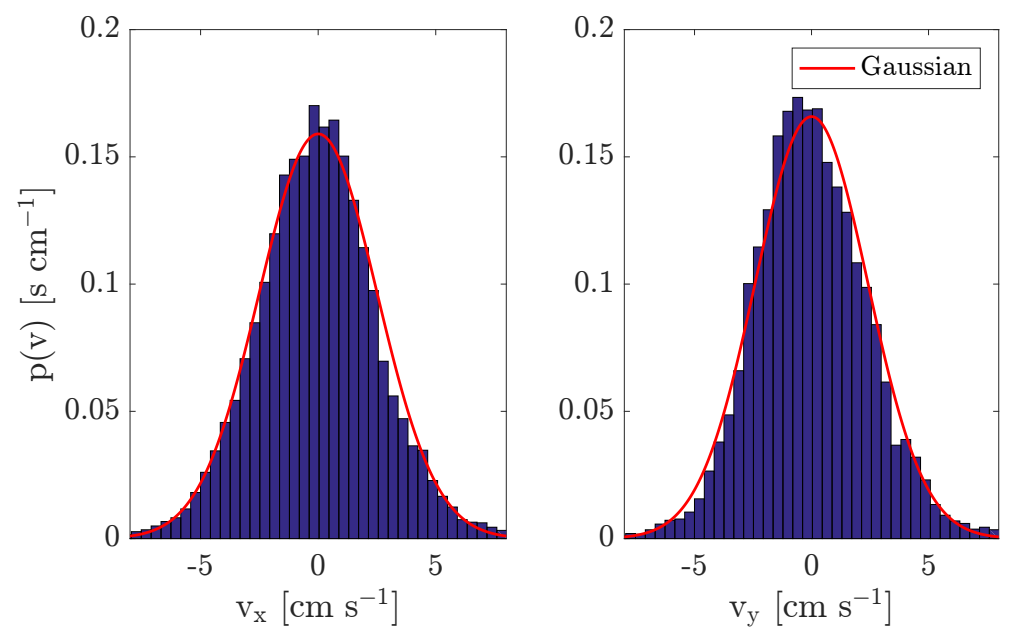

FIGURE 6.6 - Distribution of the $x$ - (left) and y-components (right) of the single particle velocity of figure 6.3-right.

trajectories smoothens the data and decreases the standard error in estimating the true $\left\langle X^{2}\right\rangle$, but also decreases the observation time.

From figure 6.7 it can be seen that the trajectories start off with a ballistic regime, characterised by a quadratic curve for $t<0.5 \mathrm{~s}$, before entering a linear regime. The curves enter a saturation regime after roughly $4 \mathrm{~s}$. The latter transition is in accordance with the confined random walk principle (Perrin, 1909; Smoluchowski, 1916). The diffusion coefficient, calculated by determining the slope of the linear regime using a $\chi$-square fitting method, varies from approximately 0.5 to $5 \mathrm{~cm}^{2} \mathrm{~s}^{-1}$.

\subsubsection{Relative translational diffusion}

As mentioned, in self-assembling systems the differential motion of the particles allows their spatial collisions and interactions. To characterise how particles move with respect to one another in the shaken reactor, we define a measure of relative diffusion between two particles $d_{\text {rel }}$ as the change in distance vector $\mathbf{d}(i)$ with respect to the initial distance $\mathbf{d}(0)$ :

$$
d_{\text {rel }}(i)=|\mathbf{d}(i)-\mathbf{d}(0)|
$$

where $\mathbf{d}(i)=\left[x_{1}(i)-x_{2}(i) y_{1}(i)-y_{2}(i)\right], x_{n}$ and $y_{n}$ being the coordinates of particle $n$ in the shaker frame. We calculate the mean square displacements from these values. figure 6.7 shows the result of applying these metrics to 3 particles in the reactor. The curves have a similar shape to the standard diffusion curves, but have a higher magnitude. The relative diffusion coefficient approx- 
imately varies from 1 to $8 \mathrm{~cm}^{2} \mathrm{~s}^{-1}$. Qualitative inspection suggests the relative diffusion equals roughly the sum of the diffusion of the individual particles, as it might be expected from a Brownian diffusion model (Gillespie and Seitaridou, 2012), though the relationship was not quantitatively investigated.

\subsubsection{Rotational diffusion}

We investigated whether the angular orientation of particles follows a random walk-like behaviour by observing their angular displacement over time. For this purpose, the values of the angular orientation $\theta$, normally restricted to the interval $-\pi$ to $\pi$, were unwrapped to a continuous, unbounded value. The result of this conversion is shown in figure 6.8.

Similarly to the displacement trajectories, the total angular trajectory was subdivided into shorter trajectories, from which we calculated the mean absolute and the mean squared angular displacement. The latter might be expected to follow (Einstein, 1905):

$$
\left\langle\theta^{2}\right\rangle=2 D_{\mathrm{r}} t
$$

where $D_{\mathrm{r}}$ is the rotational diffusion coefficient, and the scalar factor 2 follows from the single angular degree of freedom available to the particles. The calculated mean absolute angular displacement, shown in figure 6.7, appears to grow linearly with time, in contrast to what the Eq. 6.4 predicts. Closer observation shows that the particles tend to rotate in a single direction with approximately fixed rate on long-term scale; and they do not often undergo random rotation direction change, as typical of random walks. This confirms that the angular rotation of the particles does not follow a simple diffusive model.

\subsection{Conclusions}

We studied the kinetics of centimetre-sized, orbitally-shaken particles by recording and analysing their 2D-constrained motion in a bounded space. Our results show that the particles possess Rayleigh-like distributed velocities in addition to the constant orbital motion components globally forced by the external actuation. Orthogonal 1D projections of particle velocity follow a Gaussian-like distribution. The parameter characterising the $x$-projected velocity distribution agrees with the corresponding parameter for the Rayleigh distribution within error bars, but neither of them are close to the parameter for the $y$-velocity distribution. The mean square displacement of the particles obeys a confined random walk model, characterised by the sequence of ballistic, linear and saturating regimes respectively for short, medium and long observation times, which is expected given the presence of hard boundaries to particle motion. The relative diffusion coefficient appears approximately equal to the sum of the diffusion coefficient of the particles. Conversely, the angular particle displacement appears to follow a super-diffusive model. 

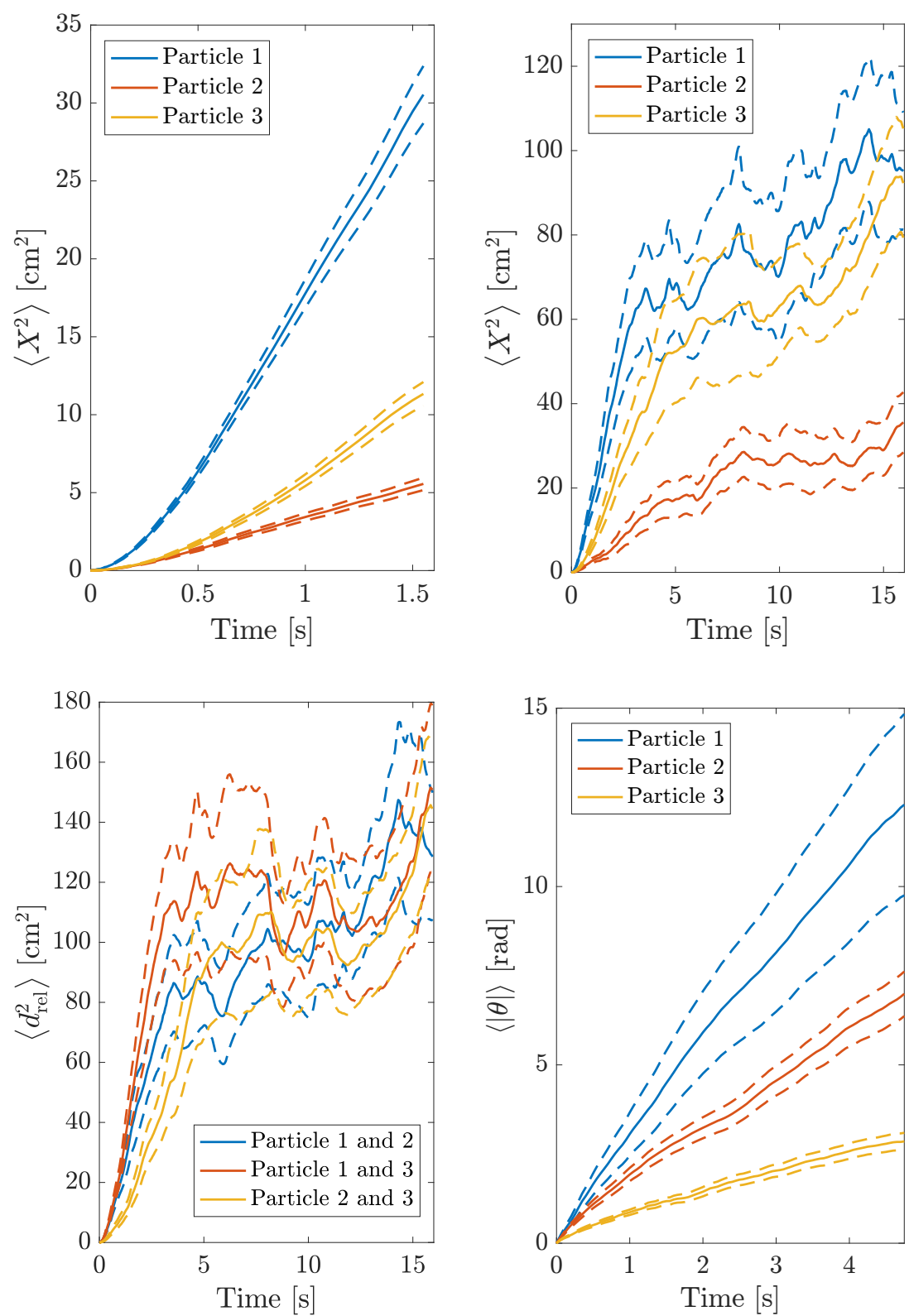

FIGURE 6.7 - Kinetics of 3 particles in the reactor. Top left: Mean square displacement as a function of time $(\leq 1.5 \mathrm{~s})$. The profile is indicative of the ballistic regime for $t \leq 0.5 \mathrm{~s}$. Top right: Mean square displacement as a function of time $(\leq 15 \mathrm{~s})$. Bottom left: Mean square relative displacement as function of time ( $\leq 15 \mathrm{~s}$ ). Bottom right: Mean absolute angular displacement as a function of time ( $\leq 5 \mathrm{~s}$ ). In all plots, dashed lines indicate the standard error around the mean value. 

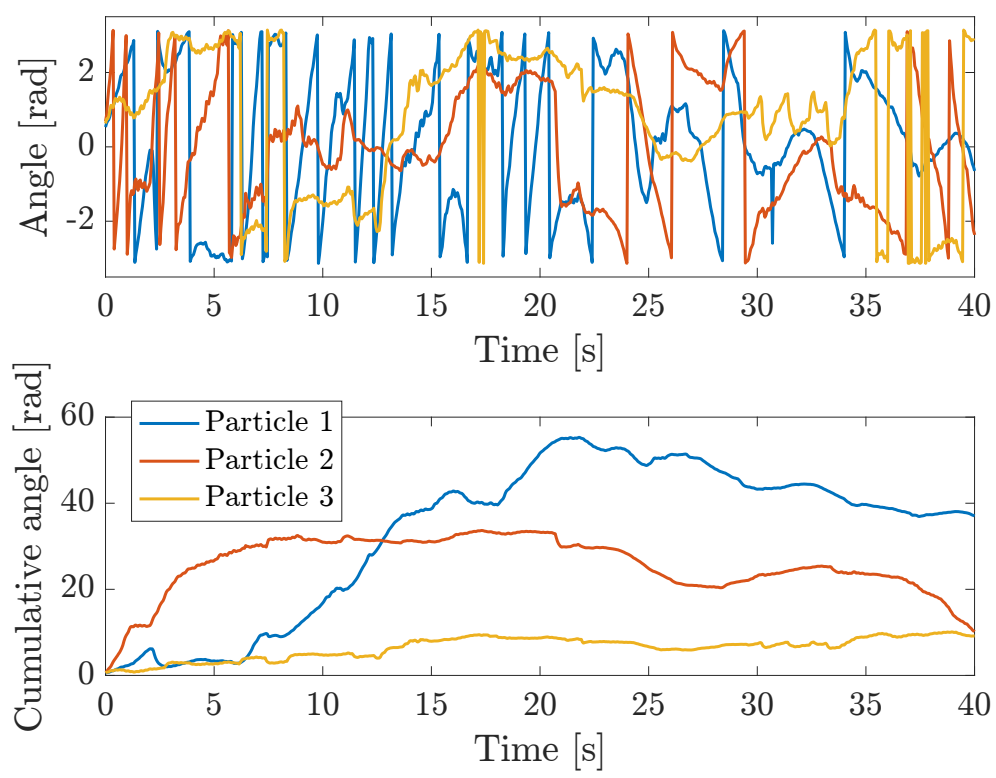

FIGURE 6.8 - Conversion of bounded to unbounded angles for three particles in the reactor.

The analogy with diffusional kinetics qualitatively supported by the results of our analysis is particularly striking when considering that the mechanism underlying the statistics of our particles' motion in the reactor is significantly different from that of e.g. molecules in a solvent. In particular, discounting for the impact of air molecules, our particles are not impinged by numerous collisions from other, smaller particles, which conversely defines simple Brownian diffusion (Gillespie and Seitaridou, 2012). We hypothesize that the origin of the particle motion may be related to frictional properties of the reactor, which could be altered or tailored by specific patterning. Future work will additionally investigate the kinetics of denser particle systems, outside of the analogy with a rarefied molecular system. 


\title{
Chapter 7
}

\section{Magnetic response of Magnetospirillum Gryphiswaldense}

\begin{abstract}
In this study we modelled and measured the U-turn trajectories of individual magnetotactic bacteria under application of rotating magnetic fields, ranging in amplitude from 1 to $12 \mathrm{mT}$. The model is based on the balance between rotational drag and magnetic torque. For accurate verification of this model, bacteria were observed inside $5 \mu \mathrm{m}$ high microfluidic channels, so that they remained in focus during the entire trajectory. From analysis of hundreds of trajectories and accurate measurements of bacteria and magnetosome chain dimensions, we confirm that the model is correct within measurement error. The resulting average rate of rotation of Magnetospirillum Gryphiswaldense is $0.74(3) \mathrm{rad} / \mathrm{mTs}$.

The work in this chapter is a team effort. My contribution includes composing the theory on drag torque and U-turns, designing and executing the the U-turn simulations, building and characterising the magnetic manipulation setup and designing the 3D printed bacteria model. I carried out the microscopy-based U-turn experiments and wrote software to automatically detect and track bacteria in the resulting videos. I furthermore carried out analysis on the videos to obtain statistical data on the velocity and length distributions, as well as on the magnetic response of the bacteria. The theory on field dependence, demagnetization factor and low field approximation was designed by Leon Abelmann and Marc Pichel. Marc Pichel also did cultivation of magnetotactic bacteria, analysis of the growth medium and design of the microfluidic chips. He carried out the macroscopic drag experiment and did SEM and TEM imaging and corresponding analysis.
\end{abstract}




\subsection{Introduction}

Magnetotactic bacteria $\left(\mathrm{MTB}^{*}\right)$ possess an internal chain of magnetosome vesicles (Komeili et al., 2004) which biomineralise nanometer sized magnetic crystals $\left(\mathrm{Fe}_{3} \mathrm{O}_{4}\right.$ or $\mathrm{Fe}_{3} \mathrm{~S}_{4}$ (Baumgartner and Faivre, 2011; Lins et al., 2005; Uebe and Schüler, 2016)), encompassed by a membrane (magnetosome) (Gorby et al., 1988). This magnetosome chain (MC) acts much like a compass needle. The magnetic torque acting on the MC aligns the bacteria with the earth magnetic field (Erglis et al., 2007). This is a form of magnetoception (Kirschvink et al., 2001), working in conjunction with aero-taxis (Frankel et al., 1997). At high latitudes the earth's magnetic field is not only aligned North-South, but also substantially inclined with respect to the earth's surface (Maus et al., 2010). The MTB are therefore aligned vertically, which converts a three-dimensional search for the optimal (oxygen) conditions into a more efficient one-dimensional search (Esquivel and Lins de Barros, 1986) (gravitational forces do not play a significant role at the scale of a bacterium). This gives MTB an evolutionary advantage over non-magnetic bacteria in environments with stationary chemical gradients more or less perpendicular to the water surface.

In this paper we address the question of how the MTB of type Magnetosprilillum Gryphiswaldense (MSR-1) respond to varying magnitudes of the external field, in particular a field that is rotating. Even though the response of individual magneto-tactic bacteria to an external magnetic field has been modelled and observed (Bahaj and James, 1993; Bahaj et al., 1996; Cebers, 2011; Erglis et al., 2007; van Kampen, 1995), there has been no thorough observation of the dependence on the field strength. The existing models predict a linear relation between the angular velocity of the bacterium and the field strength, but this has not been confirmed experimentally. Nor has there been an analysis of the spread in response over the population of bacteria. The main reason for the absence of experimental data is that the depth of focus at the magnification required prohibits the observation of multiple bacteria in parallel. In this paper, we introduce microfluidic chips with a channel depth of only $5 \mu \mathrm{m}$, which ensures that all bacteria in the field of view remain in focus.

The second motivation for studying the response of MTB to external magnetic fields, is that they are an ideal model system for self propelled medical microrobotics (Abbott et al., 2009; Menciassi et al., 2007). Medical micro-robotics is a novel form of minimally invasive surgery (MIS), in which one tries to reduce the patient's surgical trauma while enabling clinicians to reach deep seated locations within the human body (Abayazid et al., 2013; Felfoul et al., 2016; Nelson et al., 2004).

The current approach in medical micro-robotics is to insert the miniaturized tools needed for a medical procedure into the patient through a small insertion or orifice. By reducing the size of these tools a larger range of natural

${ }^{*}$ Throughout this thesis we will use the acronym MTB to indicate the single bacterium as well as multiple bacteria 
pathways becomes available. Currently, these tools are mechanically connected to the outside world. If this connection can be removed, so that the tools become untethered, (autonomous) manoeuvring through the veins and arteries of the body becomes possible (Dankelman et al., 2011).

If the size and/or application of these untethered systems inside the human body prohibits the storage of energy for propulsion, the energy has to be harvested from the environment. One solution is the use of alternating magnetic fields (Abbott et al., 2009). This method is simple, but although impressive progress has been made, it is appallingly inefficient. Only a fraction, $10^{-12}$, of the supplied energy field is actually used by the micro-robot. This is not a problem for microscopy experiments, but will become a serious issue if the micro-robots are to be controlled deep inside the human body. The efficiency would increase dramatically if the micro-robot could harvest its energy from the surrounding liquid. In human blood, energy is abundant and used by all cells for respiration.

For self-propelled objects, only the direction of motion needs to be controlled by the external magnetic field. There is no need for field gradients to apply forces, so the field is allowed to be weaker and uniform when solely using magnetic torque (Nelson et al., 2010). Compared to systems that derive their energy for propulsion from the magnetic field, the field can be small in magnitude and only needs to vary slowly. As a result, the energy requirements are low and overheating problems can be avoided.

Nature provides us with a plenitude of self-propelled micro-organisms that derive their energy from bio-compatible liquids, as described first by Bellini (1963). MTB provide a perfect biokleptic model to test concepts and study the behaviour of self-propelled micro-objects steered by external magnetic fields (Khalil et al., 2013).

The direction of the motion of an MTB is modified by the application of a magnetic field at an angle with the easy axis of magnetization of the magnetosome. The resulting magnetic torque causes a rotation of the MTB at a speed that is determined by the balance between the magnetic torque and the rotational drag torque. Under the application of a uniform rotating field, the bacteria follow U-turn trajectories (Bahaj and James, 1993; Reufer et al., 2014; Yang et al., 2012).

The magnetic torque is often modelled by assuming that the magnetic element is a permanent magnet with dipole moment $\boldsymbol{m}\left[\mathrm{Am}^{2}\right]$ on which the magnetic field $\boldsymbol{B}[\mathrm{T}]$ exerts a torque $\boldsymbol{\Gamma}=\boldsymbol{m} \times \boldsymbol{B}[\mathrm{Nm}]$. This simple model suggest that the torque increases linearly with the field strength, where it is assumed that the atomic dipoles are rigidly fixed to the lattice, and hardly rotate at all. This is usually only the case for very small magnetic fields.

In general one should consider a change in the magnetic energy as a function of the magnetization direction with respect to the object (magnetic anisotropy). This is correctly suggested by Erglis et al. (2007) for magnetotactic bacteria. An estimation of the magnetic dipole moment can be obtained by studying the dynamics of MTB (Bahaj et al., 1996). 
Recent studies of the dynamics of MTB in a rotating magnetic field show that a random walk is still present regardless of the presence of a rotating field (Cebers, 2011; Smid et al., 2015). The formation and control of aggregates of MTB in both two- and three-dimensional control systems has been achieved in vitro (De Lanauze et al., 2014; Martel and Mohammadi, 2010; Martel et al., 2009) as well as in vivo (Felfoul et al., 2016), showing that MTB can use the natural hypoxic state surrounding cancerous tissue for targeted drug delivery.

Despite these impressive results, successful control of individual MTB is much less reported. This is because many experiments suffer from a limited depth of focus of the microscope system, leading to a loss of tracking. A collateral problem is overheating of the electromagnets in experiments that take longer than a few minutes. We recently demonstrated the effect of varying field strengths on the control of magneto-tactic bacteria (Hassan et al., 2016). In the present paper we provide the theoretical framework and systematically analyse the influence of the magnetic field on the trajectories of individual MTB. This knowledge will contribute to more efficient control of individual MTB, and ultimately self-propelled robotic systems in general.

We present a thorough theoretical analysis of the magnetic and drag torques on MTB. This model is used to derive values for the proportionality between the average rate of rotation and the magnetic field during a U-turn trajectory under a magnetic field reversal. The theory is used to predict U-turn trajectories of MTB, which are the basis for our experimental procedures.

Lastly, we present statistically significant experimental results which verify our theoretical approach and employ a realistic range of magnetic field strength and rotational speed of the applied magnetic field to minimize energy input.

\subsection{Theory}

\subsubsection{The Rate of Rotation}

\section{The dependence on the field}

The magnetic torque $\Gamma[\mathrm{Nm}]$ is equal to the change in total magnetic energy $U[\mathrm{~J}]$ with changing applied field angle. We consider only the demagnetization and external field energy terms. The demagnetization energy is caused by the magnetic stray field $\boldsymbol{H}_{\mathrm{d}}[\mathrm{A} / \mathrm{m}]$ that arises due to the magnetosome magnetization $M[\mathrm{~A} / \mathrm{m}]$. In principle, one has to integrate the stray field over all space. Fortunately, this integral is mathematically equivalent to (Hubert and Schäfer, 1998)

$$
U_{\mathrm{d}}=\frac{1}{2} \mu_{0} \int \boldsymbol{M} \cdot \boldsymbol{H}_{\mathrm{d}} \mathrm{d} V,
$$

with $\mu_{o}$ the vacuum permeability, $4 \pi 10^{-7}$. In this formulation, the integral is conveniently restricted to the volume $V$ of the magnetic material.

The demagnetization energy acts to orient the magnetization so that the external stray field energy is minimized. We can define a shape anisotropy term 


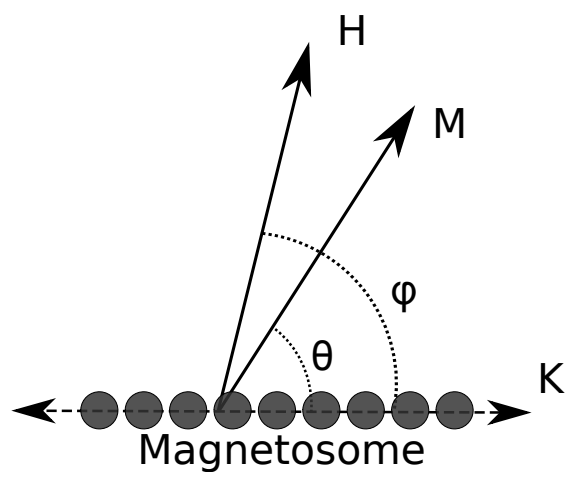

FIGURE 7.1 - Definition of the field angle $\varphi$ and the magnetization angle $\theta$ between the easy axis $K$, the magnetization $M$ and the magnetic field $H$.

$K\left[\mathrm{~J} / \mathrm{m}^{3}\right]$ to represent the energy difference between the hard and easy axes of magnetization, which are perpendicular to each other,

$$
K=\left(U_{\mathrm{d}, \max }-U_{\mathrm{d}, \min }\right) / V .
$$

The external field energy is caused by the externally applied field $\boldsymbol{H}[\mathrm{A} / \mathrm{m}]$

$$
U_{\mathrm{H}}=-\mu_{0} \int \boldsymbol{M} \cdot \boldsymbol{H} \mathrm{d} V,
$$

and acts to align $\boldsymbol{M}$ parallel to $\boldsymbol{H}$. Assuming that the magnetic element of volume $V$ is uniformly magnetized with saturation magnetization $M_{\mathrm{s}}[\mathrm{A} / \mathrm{m}]$, the total energy can then be expressed as

$$
U=K V \sin ^{2}(\theta)-\mu_{0} M_{\mathrm{S}} H V \cos (\varphi-\theta) .
$$

The angles $\theta$ and $\varphi$ are defined as in figure 7.1. Normalizing the energy, field, and torque by

$$
\begin{aligned}
u & =U / K V \\
h & =\mu_{0} H M / 2 K \\
\tau & =\Gamma / K V,
\end{aligned}
$$

respectively, the expression for the energy can be simplified to

$$
u=\sin ^{2}(\theta)-2 h \cos (\varphi-\theta) .
$$

The equilibrium magnetization direction is reached for $\partial u / \partial \theta=0$. The solution for this relationship cannot be expressed in an analytically concise form. 
The main results are however that for $h<1 / \sqrt{2}$, the maximum torque is reached at the field angle $\varphi_{\max }=\pi / 2$,

$$
\begin{aligned}
\tau_{\max } & =2 h \sqrt{1-h^{2}} & & \text { for } h \leq 1 / \sqrt{2} \\
& =1 & & \text { for } h>1 / \sqrt{2} .
\end{aligned}
$$

The angle of magnetization at maximum torque can be approximated by

$$
\theta_{\max }=h+0.1 h^{2} \text { for } h<1 / \sqrt{2},
$$

where the error is smaller than $5 \times 10^{-3} \operatorname{rad}\left(1.6^{\circ}\right)$ for $h<0.5$.

For $h>1$, the field angle $\varphi_{\max }$ at which the maximum torque is reached is smaller than $\pi / 2$ and approaches $\pi / 4$ for $h \rightarrow \infty$. This behaviour can be very well approximated by

$$
\varphi_{\max }=\frac{\pi}{4}\left(1+\frac{2}{3 h}\right) \text { for } h>1,
$$

where the error is smaller than $3 \times 10^{-3} \pi\left(0.5^{\circ}\right)$.

In summary, and returning to variables with units, the maximum torque is $\Gamma_{\max }=K V$, which is reached at

$$
H>\frac{\sqrt{2} K}{\mu_{0} M_{\mathrm{s}}}
$$

at an angle $\varphi=\pi / 2$, which, to a good approximation, decreases linearly with $1 / H$ to $\varphi=\pi / 4$ at an infinite external field.

\section{Demagnetization factor}

The magnetization $M_{\mathrm{s}}$ is a material parameter, so the only variable to be determined is the magnetosome's demagnetization factor. As a first approximation, we can consider the chain of magnetic crystals in the magnetosome as a chain of $n$ dipoles separated at a distance $a$, each with a dipole moment $m=M_{s} V\left[\mathrm{Am}^{2}\right]$, where $V$ is the volume of each single sphere. We assume that all dipoles are aligned parallel to the field $(\varphi=\theta)$ to obtain an upper limit on the torque. (See figure 7.1 for the definition of the angles). The magnetic energy for such a dipole chain has been derived by Jacobs and Bean (1955) which, rewritten in SI units, is

$$
\begin{aligned}
U= & \frac{\mu_{0} m^{2}}{4 \pi a^{3}} n K_{n}\left(1-3 \cos ^{2}(\theta)\right)+ \\
& \mu_{0} n m H \cos (\varphi-\theta) \\
K_{n}= & \sum_{j=1}^{n} \frac{(n-j)}{n j^{3}} .
\end{aligned}
$$


The maximum torque equals the energy difference between the state where all moments are parallel to the chain $(\theta=0)$ and the state where they are perpendicular to the chain $(\theta=\pi / 2)$, under the condition that the angle between the moments and the field is zero:

$$
\Gamma_{\max }=\frac{3 \mu_{0} m^{2}}{4 \pi a^{3}} n K_{n} .
$$

For a single dipole $n=1, K_{n}=0$ and there is no energy difference, as expected.

Combined with equations (7.6) and (7.9), and re-introducing units, the field dependent torque becomes

$$
\begin{aligned}
\Gamma & =\Gamma_{\max } 2 h \sqrt{1-h^{2}} \text { with } \\
h & =\frac{H}{\Delta N M_{\mathrm{s}}} .
\end{aligned}
$$

The magnetosome does not consist of point dipoles but should be approximated by spheres with radius $r$, spaced at a distance of $a$ from each other (figure 7.2). We can modify the Jacob and Bean model by introducing the volume of a single sphere $V$ and the magnetization $M_{s}$ of the magnetite crystal $\left(4.8 \times 10^{5} \mathrm{~A} / \mathrm{m}\right.$ (Witt et al., 2005)),

$$
\begin{gathered}
\Gamma_{\max }=\frac{1}{2} \mu_{0} M_{s}^{2} n V \Delta N \\
\Delta N=2 K_{n}\left(\frac{r}{a}\right)^{3},
\end{gathered}
$$

as a correction to equation 7.16. This correction is based on the fact that the field of a uniformly magnetized sphere is identical to a dipole field (Griffiths, 1999) outside the sphere, and the average of the magnetic field over a sphere not containing currents is identical to the field at the center of that sphere $(\mathrm{Hu}$, 2000, 2008).

For an infinitely long chain of touching spheres, $d=0$ and $n \rightarrow \infty$, the difference in demagnetization factors $(\Delta N)$ approaches 0.3 (Figure 7.3). Approximating the chain by a long cylinder $(\Delta N=0.5)$ (Erglis et al., 2007; Hanzlik et al., 1996) therefore overestimates the maximum torque by $40 \%$. Simply taking the total magnetic moment to calculate the torque, as if $\Delta N=1$, would overestimate it by a factor of three.

\section{Low field approximation}

For low values of the field $(h \ll 1)$, equation (7.16) can be approximated by

$$
\Gamma \approx \Gamma_{\max } 2 h=\mu_{0} M_{\mathrm{s}} n V H=m B,
$$




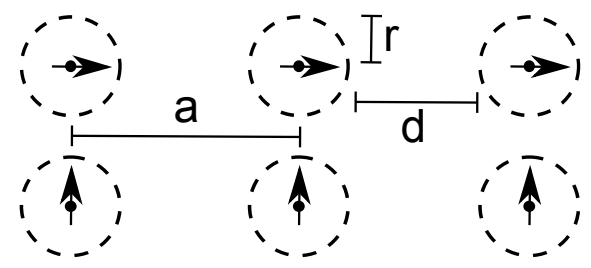

FIGURE 7.2 - Chain of magnetic spheres of radius $r$, spaced at a distance $d$, approximated by point dipoles spaced by a distance $a=r+d$, magnetized along the longitudinal axis of the chain (top) or perpendicular to its longitudinal axis (bottom).

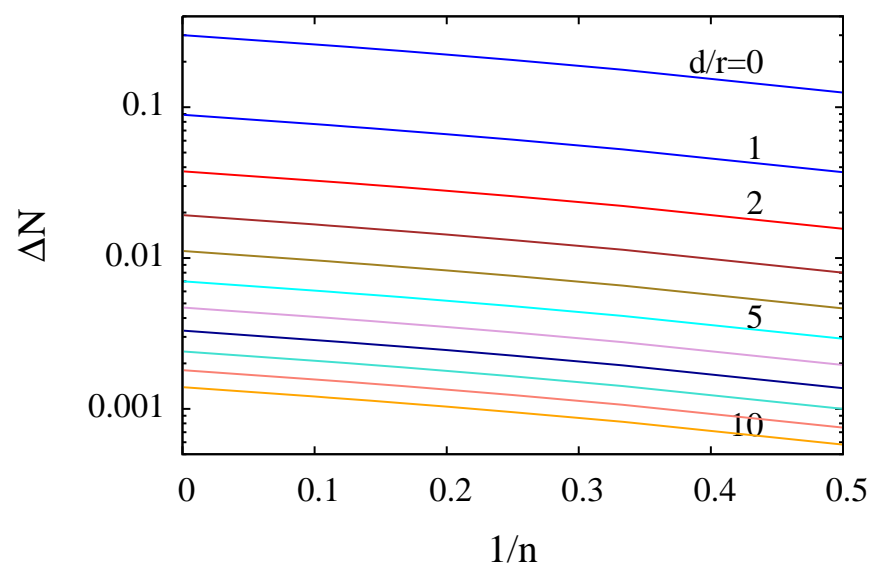

FIGURE 7.3 - Difference in demagnetization factors of a chain of spheres as function of number of spheres $n$ for varying spacing between the spheres $d / r$.

where $m\left[\mathrm{Am}^{2}\right]$ is the total magnetic moment of the magnetosome chain and assuming the permeability of the medium to be equal to vacuum. This approximation is commonly used in the field of MTB studies. Based on the theory presented here, it is now possible to estimate up to which field value this is approximation is valid.

The normalization to the reduced field $h$ is solely dependent on the magnetization and demagnetization factors of the chain. Based on the values for magnetosome morphology (table 7.1), we can estimate the field dependence of the torque. Figure 7.4 shows the torque as a function of the field for the range of values tabulated, normalized to the maximum torque. Also shown is the approximation for the case when the magnetization remains aligned with the easy axes. For Magnetospirillum Gryphiswaldense, the linear range is valid up to fields of 


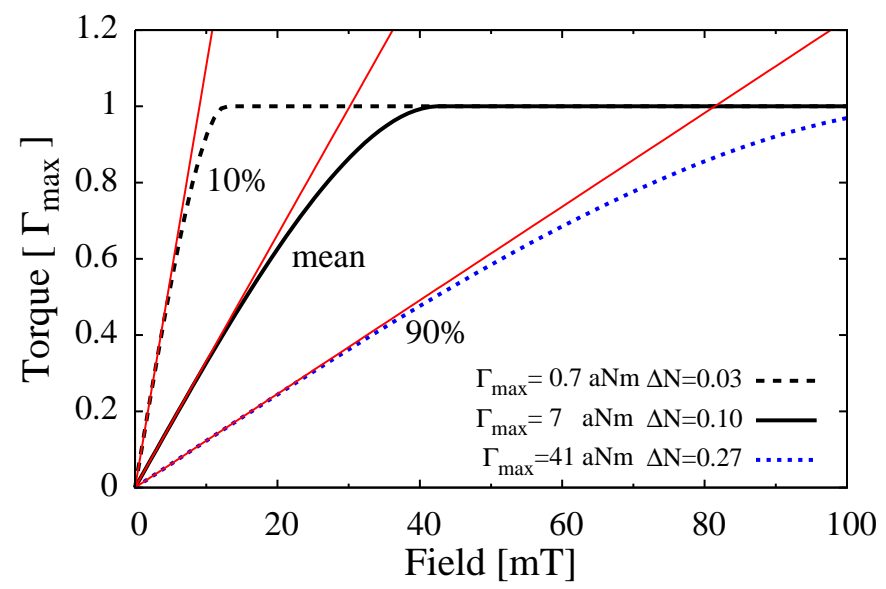

FIGURE 7.4 - Magnetic torque on magnetotactic bacteria, normalized to the maximum torque, as a function of applied field for the average of the population, as well as the $10 \%$ and $90 \%$ cut-off (see table 7.1). The red solid asymptotes show the linear approximation for $\Gamma=m B$.

about $10 \mathrm{mT}$ for $90 \%$ of the population.

\section{Drag torque}

Magnetotactic bacteria are very small, and rotate at a few revolutions per second only. Inertial forces therefore do not play a significant role. The ratio between the viscous and inertial forces is characterized by the Reynolds number $R e$, which for rotation at an angular velocity of $\omega[\mathrm{rad} / \mathrm{s}]$ is

$$
R e=\frac{L^{2} \rho \omega}{4 \eta},
$$

where $L$ is the characteristic length (in our case, the length of the bacterium), $\rho$ the density, and $\eta$ the dynamic viscosity of the liquid (for water, these are, respectively, $10^{3} \mathrm{~kg} / \mathrm{m}^{3}$, and $1 \mathrm{mPas}$ ). Experiments by Dennis et al. (1980) show that a Stokes flow approximation for the drag torque is accurate up to $R e=10$. In experiments with bacteria, the Reynolds number is on the order of $10^{-3}$ and the Stokes flow approximation is certainly allowed. The drag torque is therefore simply given by

$$
\Gamma_{\mathrm{D}}=f_{\mathrm{b}} \omega,
$$

The rotational drag coefficient of the bacterium, $f_{\mathrm{b}}$, needs to be estimated for the type of MTB studied. In a first approximation, one could consider the 
MTB to be a rod of length $L$ and diameter $W$. Unfortunately, there is no simple expression for the rotational drag of a cylinder. Dote and Kivelson (1983) give a numerical estimate of the rotational drag of a cylinder with spherical caps (spherocylinder). Fortunately, for typical MSR-1 dimensions, it can be shown that a prolate spheroid of equal length and diameter has a rotational drag coefficient that is within $10 \%$ of that value. To a first approximation, one can therefore assume the rotational drag of an MSR-1 to be given by (Berg, 1993)

$$
f_{\mathrm{e}}=\frac{\pi \eta L^{3}}{3 \ln \left(\frac{2 L}{W}\right)-\frac{3}{2}} .
$$

However, the MSR-1 has a spiral shape, so the actual drag will be higher. Rather than resorting to complex finite element simulations, we chose to empirically determine the rotational drag torque by macroscopic experiments with $3 \mathrm{D}$ printed bacteria models in a highly viscous medium (section 7.3.4.). We introduce a bacteria shape correction factor $\alpha_{\text {bs }}$ to the spheroid approximation, which is independent of the ratio $L / W$ over the range of typical values for MSR1 and has a value of about 1.65. The corrected rotational drag coefficient for the bacteria then becomes

$$
f_{\mathrm{b}}=\alpha_{\mathrm{bs}} f_{\mathrm{e}} \text {. }
$$

\section{Diameter and duration of the U-turn}

At the steady-state rate, the magnetic torque is balanced by the rotational drag torque, leading to a rate of rotation of

$$
\omega=\frac{\Gamma}{f_{\mathrm{b}}} \approx \frac{m B \sin \phi(t)}{f_{\mathrm{b}}} .
$$

The approximation is for low field values (see figure 7.4), in which case $\phi$ is the angle between the applied field and the long axis of the bacteria (magnetosome).

The maximum rate of rotation, $m B / f_{\mathrm{b}}$, is obtained when the field is perpendicular to the long axis of the bacteria. Suppose that we construct a control loop to realize this condition over the entire period of a U-turn. Then the minimum diameter and duration of this loop would be

$$
\begin{aligned}
D_{\text {min }} & =\frac{2 f_{\mathrm{b}} \nu}{m B} \\
T_{\text {min }} & =\frac{\pi f_{\mathrm{b}}}{m B},
\end{aligned}
$$

where $D_{\min }$ is the minimum size of a U-turn's diameter and $T_{\min }$ is the minimum time of a U-turn. On the other hand, if we reverse the field instantan- 
eously, the torque will vary over the trajectory of the U-turn. Compared to the situation above, the diameter of the U-turn increases by a factor of $\pi / 2$ :

$$
D=\frac{\pi f_{\mathrm{b}} v}{m B} .
$$

The diameter of the U-turn increases with the velocity of the bacterium. To obtain a description that only depends on the dimensions of the bacteria, we introduce a new parameter $v / D[\mathrm{rad} / \mathrm{s}]$, which can be interpreted as an average rate of rotation. The relation between the average rate of rotation and the magnetic field $B$ is

$$
\frac{v}{D}=\gamma B
$$

where the proportionality factor $\gamma$ [rad/Ts] can be linked to the bacterial magnetic moment $m$ and drag coefficient $f_{\mathrm{b}}$ [Nms],

$$
\gamma=\frac{m}{\pi f_{\mathrm{b}}}
$$

Note, however, that this expression is only valid in the low field approximation.

The determination of the duration of the U-turn trajectory is complicated by the fact that the magnetic torque starts and ends at zero (at $\theta=0$ or $\pi$ ). In this theoretical situation, the bacteria would never turn at all. Esquivel and Lins de Barros (1986) solved this problem by assuming a disturbance acting on the motion of the bacteria. This disturbance could be due to Brownian motion, as used by Esquivel, or due to flagellar propulsion, as we use in the simulations in the following section. Assuming an initial disturbing angle of $\theta_{\mathrm{i}}$, the duration $T$ [s] of the U-turn becomes

$$
T=\frac{2 f_{\mathrm{b}}}{m B} \ln \frac{2}{\theta_{\mathrm{i}}} .
$$

\subsubsection{U-turn trajectory simulations}

To check the validity of the analytical approach, we performed simulations. The MTB are approximated by rigid magnetic dipoles with constant lateral velocity $v$ at an orientation of $\theta_{x}(t)$ and angular velocity of $\omega(t)$ (see figure 7.5). They are subject to a magnetic field with magnitude $B$ at an orientation of $\varphi_{x}(t)$, resulting in a magnetic torque of $\Gamma(t)$. In contrast to the analytical model, it is assumed that flagellar motion causes an additive sinusoidal torque $\Gamma_{\mathrm{f}}(t)$ with amplitude $A_{\mathrm{f}}$ and angular velocity $\omega_{\mathrm{f}}$. These should be in balance with the drag torque: $\Gamma_{\mathrm{D}}=f_{\mathrm{b}} \omega(t)$. The following set of equations link the physical model to the coordinates $x(t), y(t)$ : 


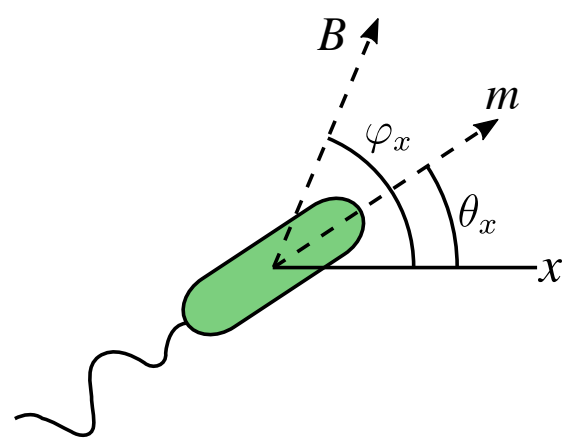

FIGURE 7.5 - Bacterium at angle $\theta_{x}$ with magnetic field at angle $\varphi_{x}$.

$$
\begin{aligned}
x(t) & = & x(0)+\int_{0}^{t} v \cos \left(\theta_{x}(t)\right) \mathrm{d} t \\
y(t) & = & y(0)+\int_{0}^{t} v \sin \left(\theta_{x}(t)\right) \mathrm{d} t \\
\theta_{x}(t) & = & \\
\omega(t) & = & \frac{1}{f_{\mathrm{b}}}\left(\Gamma_{\operatorname{mag}}(t)+\Gamma_{\text {flag }}(t)\right) \\
& = & \frac{m B}{f_{\mathrm{b}}} \sin \left(\varphi_{x}(t)-\theta_{x}(t)\right)+\frac{A_{\mathrm{f}}}{f_{\mathrm{b}}} \sin \left(\omega_{\mathrm{f}} t\right)
\end{aligned}
$$

A linear, closed-form solution of the diameter of the trajectory of the U-turn in the case of an instantaneous field reversal and no flagellar torque is given by equation 7.29. This solution is not valid, however, in the case of slowly rotating fields. The experimental magnetic field is considered to rotate according to a constant-acceleration model with a total rotation period of $130 \mathrm{~ms}$ (see section 7.3.4). Simulations were carried out with time steps of $10 \mu \mathrm{s}$, which is comfortably fast and precise (decreasing this to $1 \mu$ s changes the results by approximately $0.01 \%$ ). Figure 7.6 shows several simulated trajectories subject to fields of various magnitudes, assuming nonzero flagellar torque and realistic MTB parameters.

Figure 7.7 shows the simulated $v / D$ as a function of the field magnitude. It can be seen that during an instantaneous field reversal, the solution is nearly identical to the closed-form solution of equation 7.29. The difference is caused by the influence of flagellar torque. Introducing a field reversal time $T_{\text {mag }}$ of 


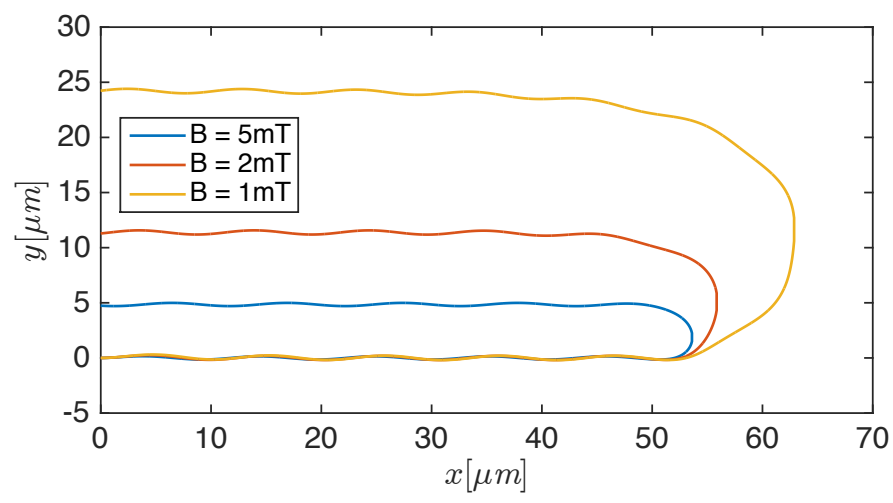

FIGURE 7.6 - Simulated trajectories assuming flagellar torque and a non-instantaneously rotating field for several values of the magnetic field magnitude $B$. The time step of the simulation is $10 \mu \mathrm{s}$

$130 \mathrm{~ms}$ into a continuous-acceleration model significantly changes the profile, yielding a similar result for low fields, increasing at moderate fields, and saturating to a maximum value of $16.6 \mathrm{~s}^{-1}$. $B_{\text {opt }}$ is defined as the field magnitude at which $v / D$ has the largest difference from the theoretical curve. Figure 7.8 shows, from simulations, that the optimal reversal time is inversely proportional to the magnetic field strength. For fields below $B_{\text {opt }}, v / D$ can be considered linear with a maximum nonlinearity error of $2 \%$, independently of $T_{\text {mag }}$.

\subsection{Experimental}

\subsubsection{Magnetotactic bacteria cultivation}

A culture of Magnetospirillum Gryphiswaldense was used for the magnetic moment study. The cultures were inoculated in MSGM medium ATCC 1653 according to with an oxygen concentration of 1 to $5 \%$. The bacteria were cultivated at $21^{\circ} \mathrm{C}$ for 2 to 5 days for optimal chain growth (Katzmann et al., 2013). The sampling was done using a magnetic "racetrack" separation, as described in (Wolfe et al., 1987).

\subsubsection{Dynamic viscosity of growth medium}

The kinematic viscosity of the freshly prepared growth medium was determined with an Ubbelohde viscometer with a capillary diameter of $0.63(1) \mathrm{mm}$ (Si Analytics 50110). The viscometer was calibrated with deionized water, assuming it has a kinematic viscosity of $0.98(1) \mathrm{mm}^{2} / \mathrm{s}$ at $21.0(5){ }^{\circ} \mathrm{C}$. At that temperature, the growth medium has a kinematic viscosity of $0.994(17) \mathrm{mm}^{2} / \mathrm{s}$. The density of the 


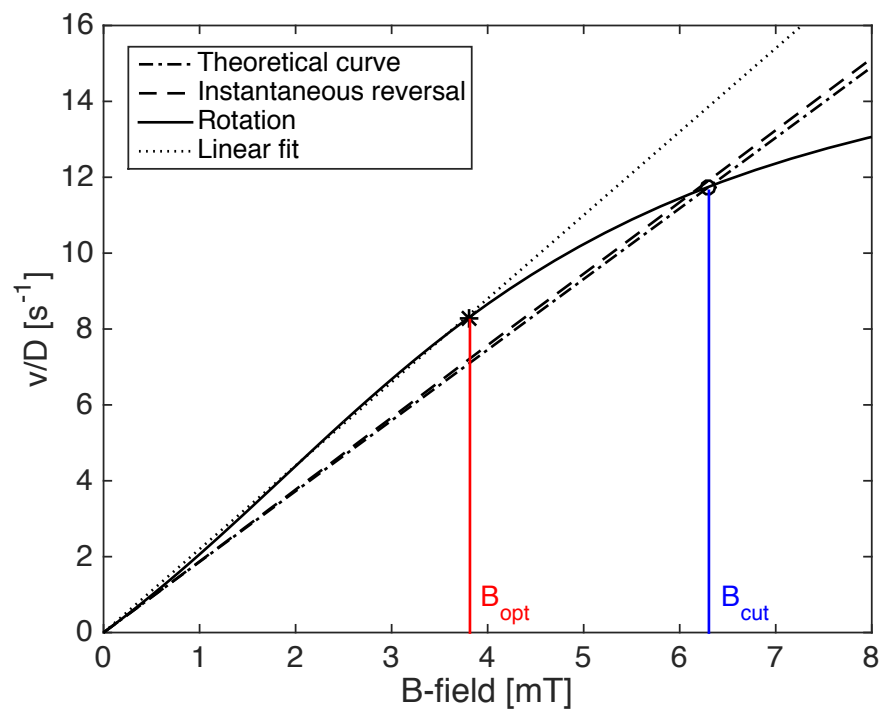

FIGURE 7.7 - Simulated values of $v / D$ for different magnetic field strengths using either instantaneous field inversion (dashed) or a rotated field (solid) compared with the theoretical linear model proposed by Erglis et al. (2007).

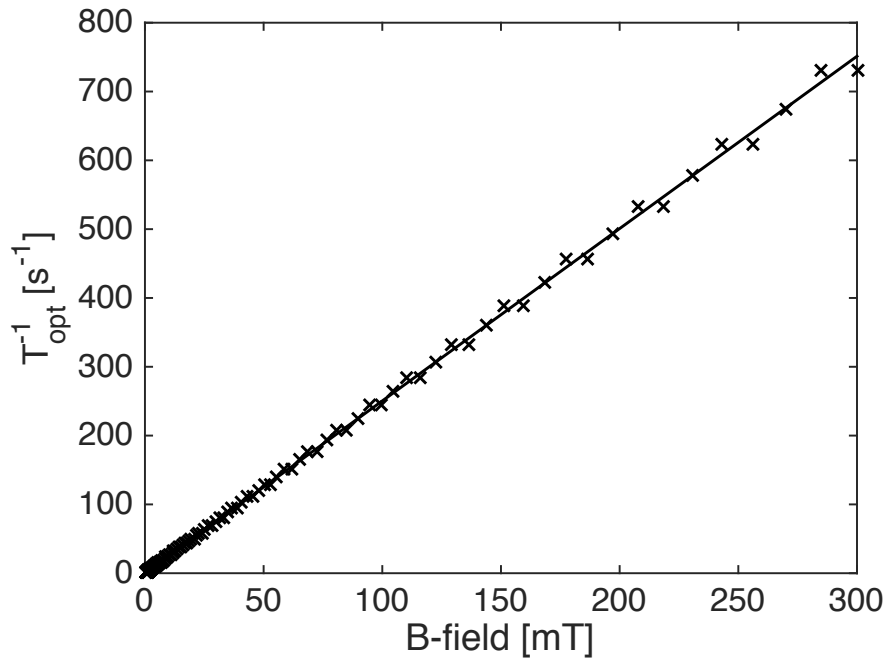

FIGURE 7.8 - Simulated optimum reversal time of the magnetic field as a function of the field strength. 


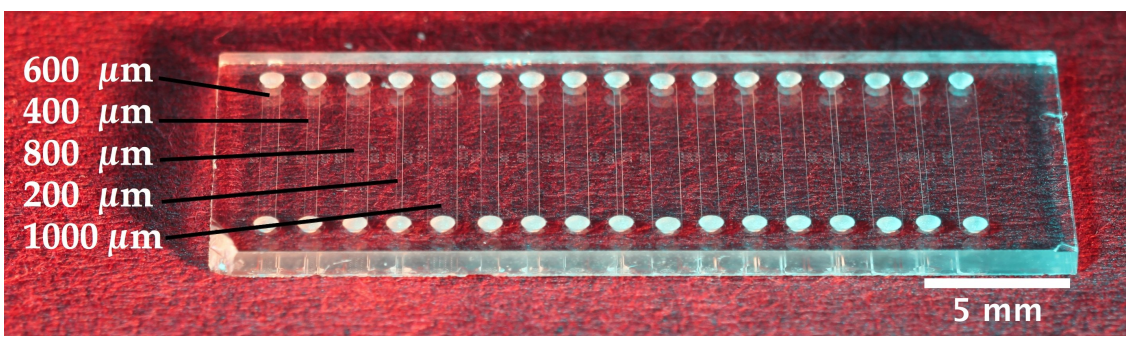

FIGURE 7.9 - Top: A $5 \mu$ m deep microfluidic chip with various channel widths of 200, 400, 600, 800 and $1000 \mu \mathrm{m}$.

growth medium was $1.009(2) \mathrm{g} / \mathrm{cm}^{3}$, measured by weighing $1 \mathrm{ml}$ of it on a balance. The dynamic viscosity of the growth medium is therefore 1.004(19) mPas, which is, within measurement error, identical to water (1.002 mPas).

\subsubsection{Microfluidic Chips}

Microfluidic chips with a channel depth of $5 \mu \mathrm{m}$ were constructed by lithography, HF etching in glass and subsequent thermal bonding. The fabrication process is identical to the one described by Park et al. (2015). Figure 7.9 shows the resulting structures, consisting of straight channels with inlets on both sides. By means of these shallow channels, the MTBs are kept within the field of focus during microscopic observation, so as to prevent out-of-plane focus while tracking. The channel width was $200 \mu \mathrm{m}$ or more, so that the area over which U-turns could be observed was only limited by the field of view of the microscope.

\subsubsection{Magnetic Manipulation Setup}

A schematic of the full setup, excluding the computer used for the acquisition of the images, is shown in figure 7.10. A permanent NdFeB magnet $(5 \times 5 \times 10 \mathrm{~mm}$, grade N42) is mounted on a stepper motor (Silverpak 17CE, Lin Engineering) below the microfluidic chip. The direction of the field can be adjusted with a precision of 51200 steps for a full rotation, at a rotation time of $130 \mathrm{~ms}$ with a constant acceleration of $745 \mathrm{rad} \mathrm{s}^{-2}$. The field strength is adjusted using a labjack, with a positioning accuracy of $0.5 \mathrm{~mm}$.

The data acquisition was done by a Flea3 digital camera $(1328 \times 1048$ at 100 fps, FL3-U3-13S2M-CS, Point Grey) mounted on a Zeiss Axiotron 2 microscope with a $20 \times$ objective.

During the experiments, a group of MTB was observed while periodically (every two seconds) rotating the magnetic field. This was recorded for field magnitudes ranging from 1 to $12 \mathrm{mT}$. Offline image processing techniques were used to track the bacteria and subtract their velocity and U-turn diameter. 


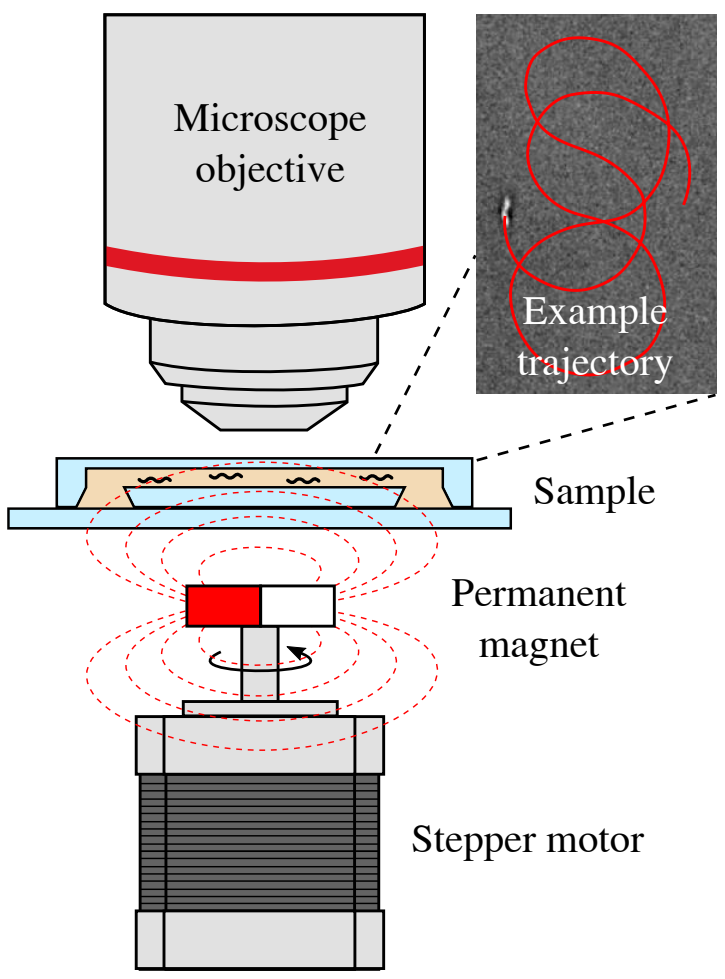

FigURE 7.10 - The setup used to measure the MTB U-turns. A reflective microscope observes the sample in the microfluidic chip. A height-adjustable motorized permanent magnet generates the magnetic fields.

Knowing the error in our measurements of the magnetic field is fundamental to determining the responsiveness of the MTB. Therefore we measured the magnetic fields at specific heights using a Hall meter (Metrolab THM1176). The results can be seen in figure 7.11.

The placement of the tip of the Hall meter was at the location of the microfluidic chip, assuming the field strength inside the chip's chamber equals that at the tip. It should be noted that the center of the magnet was aligned with the center of rotation of the motor, therefore the measurements were only done with a stationary magnet on top of an inactive motor. Errors in the estimation of the magnetic field strength due to misalignment of the magnetic center from our measurements therefore cannot be excluded.

The rotation profile of the motorized magnet was investigated by recording its motion by a digital camera at $120 \mathrm{fps}$ and evaluating its time-dependent angle by manually drawing tangent lines. Figure 7.12 shows that the profile 


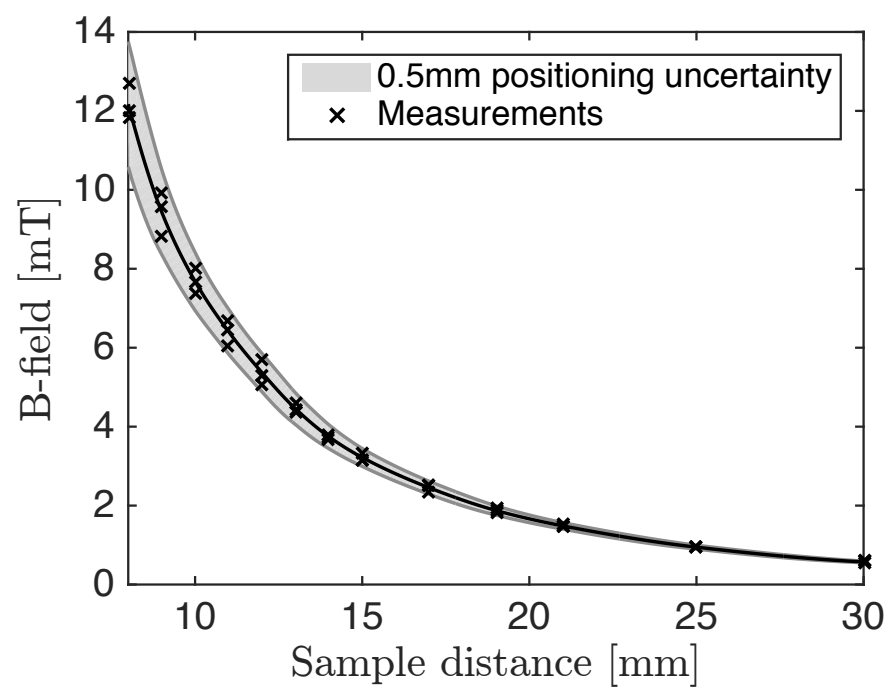

FIGURE 7.11 - Magnetic field strength as a function of distance of the magnet to the microfluidic chip.

accurately fits a constant-acceleration model with an acceleration of $745 \mathrm{rad} \mathrm{s}^{-2}$, resulting in a total rotation time of $130 \mathrm{~ms}$.

\subsubsection{Macroscopic Drag Setup}

Macro-scale drag measurements were performed using a Brookfield DV-III Ultra viscometer. During the experiment, we measured the torque required to rotate different centimetre sized models of bacteria and simple shapes in silicone oil (Figure 7.13). In order to keep the Reynolds number less than one, silicone oil of $5000 \mathrm{mPas}$ (Calsil IP 5000 from Caldic, Belgium) was used as a medium to generate enough drag. Furthermore, the parts were rotated at speeds below $30 \mathrm{rpm}$. The models were realized by $3 \mathrm{D}$ printing.

\subsubsection{Image Processing}

The analysis of the data was done using in-house detection and tracking scripts written in MATLAB. The process is illustrated in figure 7.14. In the detection step, static objects and non-uniform illumination artefacts are removed by subtracting a background image constructed by averaging 30 frames spread along the video. High-frequency noise is reduced using a Gaussian lowpass filter. A binary image is then obtained using a thresholding operation, followed by selection on a minimum and maximum area size. The centres-of-mass of the remaining 


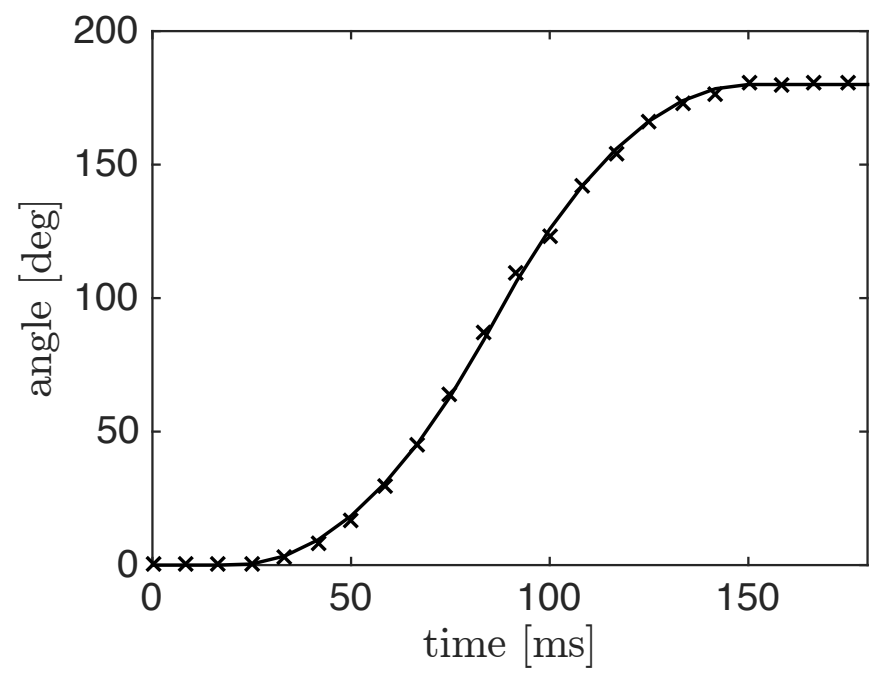

FIGURE 7.12 - The measured angle of the motorized magnet accurately fits a constant-acceleration model with a total rotation period of $130 \mathrm{~ms}$.
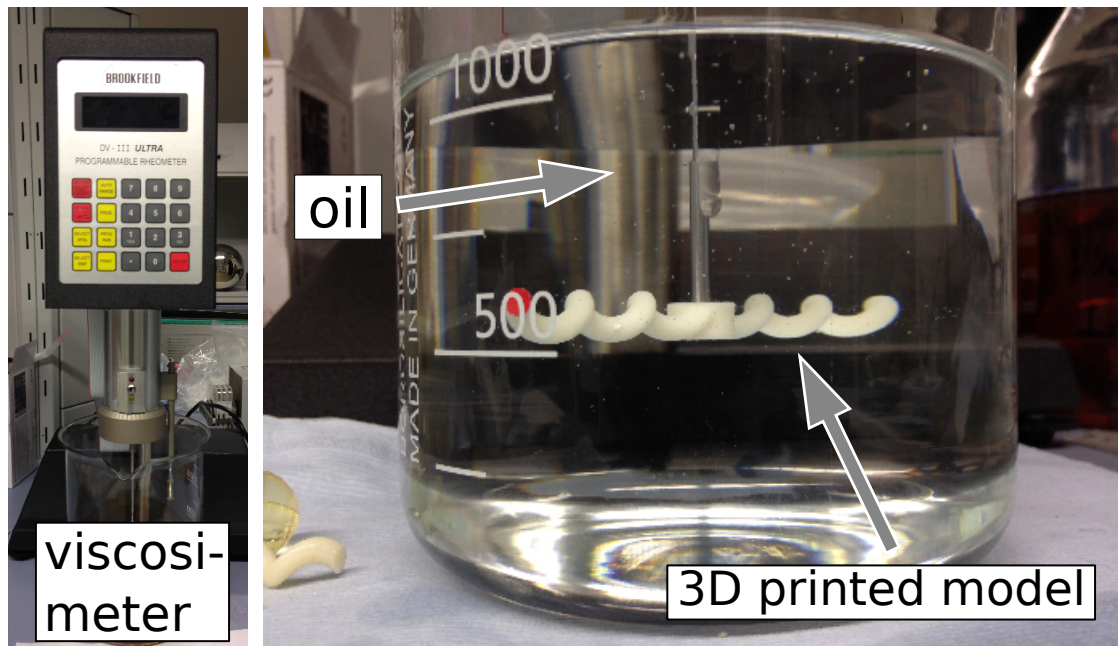

FIGURE 7.13 - The viscometer setup used to measure the rotational drag of macroscopic spheroid and helical structures. 3D printed models were mounted on a shaft and rotated in a high viscosity silicone oil (5 Pas). 


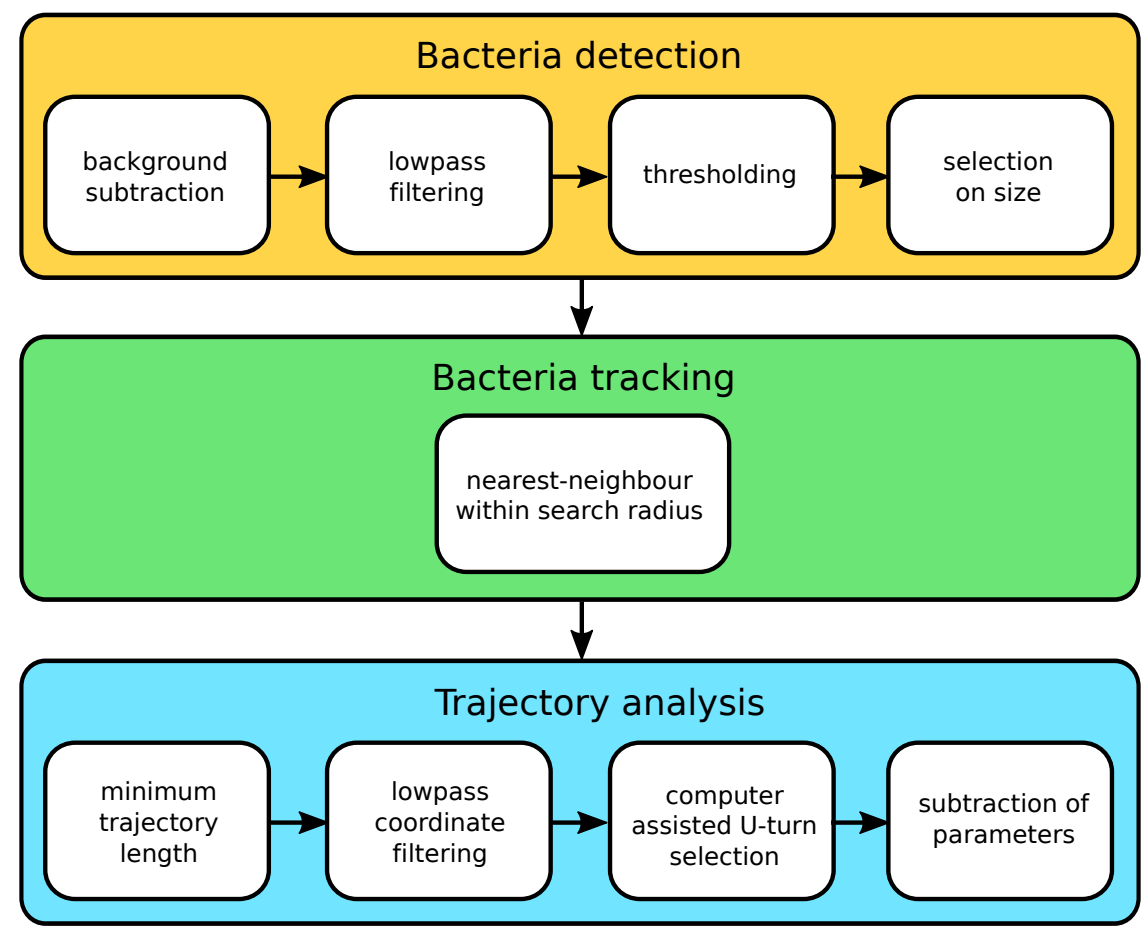

FIGURE 7.14 - The process of bacteria detection, tracking, and subsequent analysis.

blobs are compared in subsequent frames, and woven to trajectories based on a nearest-neighbour search within a search radius. A sequence of preprocessing steps can be seen in figure 7.15.

Subsequently, the post-processing step involves the semi-automated selection of the MTB trajectories of interest for the purpose of analysis. The U-turn parameters of interest analysed are the velocity $v$, the diameter $D$ of the U-turn, and the time $t$. A typical result of the post-processing step can be seen in figure 7.16.

\subsection{Results and discussions}

The model developed in section 7.2 predicts the trajectories of MTB under a changing magnetic field: in particular, the average rate of rotation over a Uturn. To validate the model, the essential model parameters are determined in section 7.4.1, after which the average rate of rotation is measured and compared to theory in section 7.4.2. 

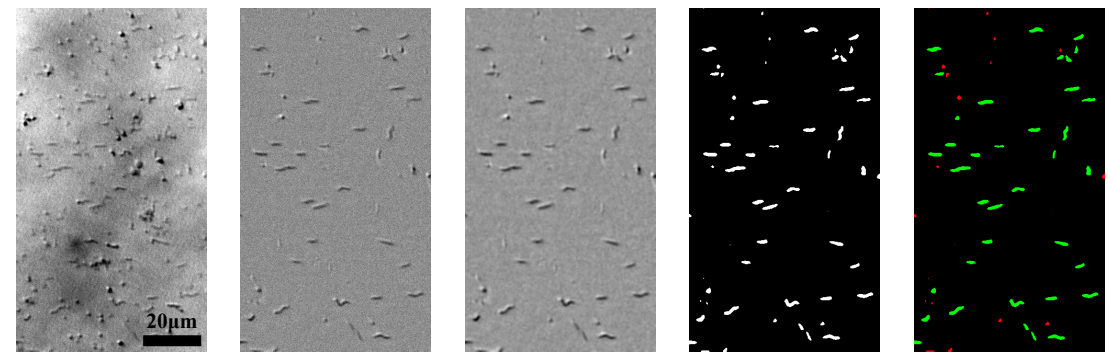

FIGURE 7.15 - Pre-processing filter steps: (a) raw, (b) background subtraction, (c) low pass filtering, (d) thresholding resulting in a binary image, (e) size selectivity.
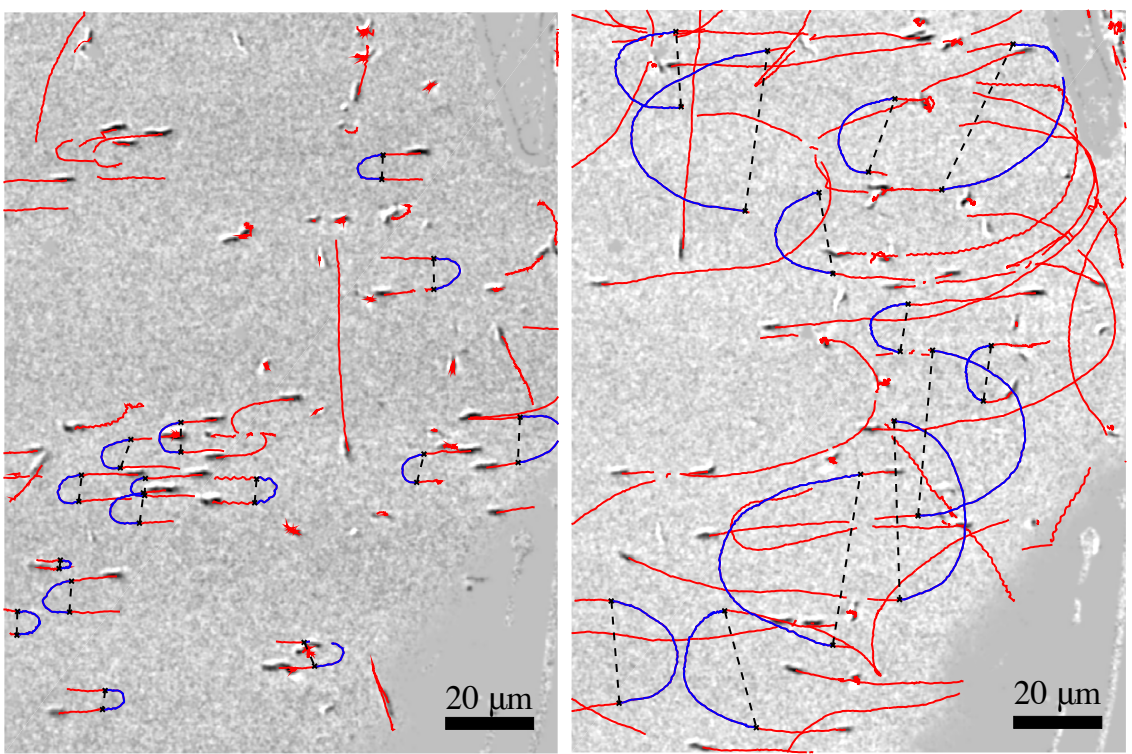

FIGURE 7.16 - Trajectories during image post-processing at a magnetic field strength of $12.2 \mathrm{mT}$ (left) and $1.5 \mathrm{mT}$ (right). The trajectory analysis highlights selected U-turns in blue and unanalysed trajectories in red. The black dashed lines connect two manually selected points of a given U-turn trajectory, from which the distance in the $y$-direction, or the U-turn diameter, is determined. 


\subsubsection{Estimate of model parameters}

The rate of rotation of an MTB under a rotating magnetic field is determined by the ratio between the rotational drag torque and the magnetic torque. Both will be discussed in the following, after which the average rate of rotation will be estimated.

\section{Estimate of rotational drag torque}

To determine the rotational drag torque, the outer shape of the MTB was measured by both optical microscopy and scanning electron microscopy (SEM). The drag coefficient was estimated from a macro-scale drag viscosity measurement.

Outer dimensions of the bacteria The length $L$ of the bacteria is measured from the same optical images as used for the trajectory analysis (figures 7.16). Scanning electron microscopy (SEM) would in principle give higher precision per bacterium, but due to the lower number of bacteria per image the estimate of the average length and distribution would have a higher error. Moreover, using the video footage ensures that the radius of curvature and the length of the bacteria are measured on the same bacterium.

A typical MTB has a length of 5.0(2) $\mu \mathrm{m}$. The length distribution is shown in Figure 7.18. These values agree with values reported in the literature (Bazylinski and Frankel, 2004; Faivre et al., 2010; Schleifer et al., 1991).

The width $W$ of the bacteria is too small to be determined by optical microscopy, and needs to be determined from SEM images, see figure 7.17. A typical bacterium has a width of 240(6) $\mathrm{nm}$. The main issue with SEM images is whether a biological structure is still intact or perhaps collapsed due to dehydration, which might cause overestimation of the width. The latter might be as high as $\pi / 2$ if the bacterial membrane has completely collapsed. Fortunately, the drag coefficient scales much more strongly with the length than with the width (equation 7.24). For a typical bacterium, the overestimation of the width by using SEM leads at most to an overestimation of the drag by $18 \%$.

Table 7.1 lists the values of the outer dimensions $L$ and $W$, including the measurement error and standard deviation over the measured population.

Rotational Drag From the outer dimensions of the bacteria, the rotational drag torque can be estimated. The bacterial shape correction factor, equation 7.25 , was determined by macro-scale experiments with 3D printed models of an MTB in a viscosimeter using high viscosity silicone oil (see section 7.3.5). Figure 7.19 shows the measured torque as a function of the rotational speed for prolate spheroids and spiral shaped 3D printed bodies of two different lengths. The relation between the torque and the speed is linear, so we are clearly in the laminar flow regime. This is in agreement with an estimated Reynolds number of less than 0.3 for this experiment (equation 7.22 ). Independently of the 


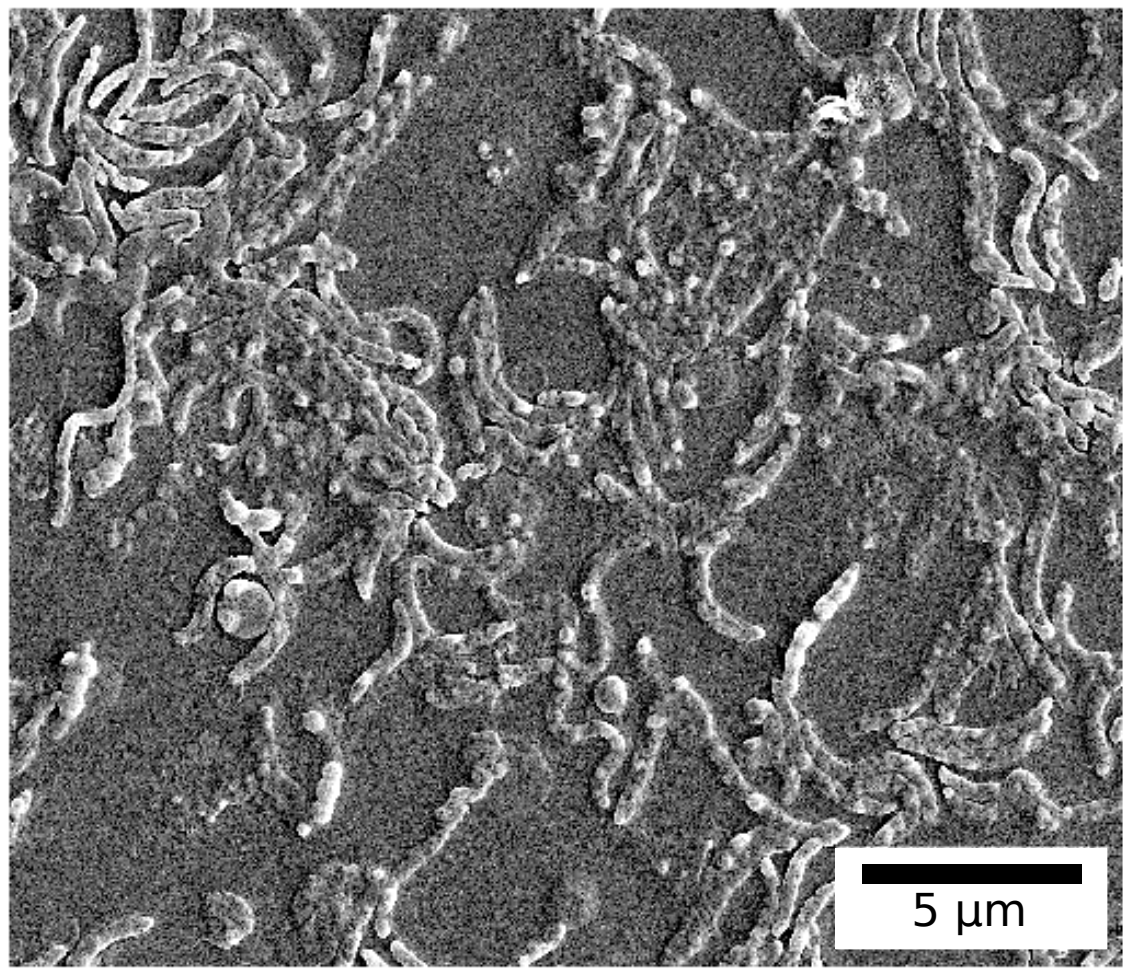

FIGURE 7.17 - Scanning electron microscopy images of Magnetospirillum Gryphiswaldense. Separated MTB were selected for width measurements.

size, the spiral shaped MTB models have a drag coefficient that is 1.64(5) times higher $\left(\alpha_{\mathrm{BS}}\right)$ than that of a spheroid of equal overall length and diameter.

Using the same experimental configuration, we can obtain an estimate of the effect of the channel walls on the rotational drag by changing the distance between the 3D printed model and the bottom of the container. Figure 7.20 shows the relative increase in drag when the spiral shape approaches the wall. This experiment was performed on a $5 \mathrm{~cm}$ long, $5 \mathrm{~mm}$ diameter spiral at $8 \mathrm{rpm}$. To visualise the increase, the reciprocal of the distance normalised to the length of the bacteria is used on the bottom horizontal axis. The normalised length is shown on the top axes. Note that when plotted in this way, the slope approaches unity at larger distances.

For an increase over $5 \%$, the model has to approach the wall at a distance smaller than $L / 3$, where $L$ is the length of the bacteria. For very long bacteria of $10 \mu \mathrm{m}$, this distance is already reached in the middle of the $5 \mu \mathrm{m}$ high channel. Since there are two channel walls on either side at the same distance, we estimate that the additional drag for bacteria swimming in the centre of the 


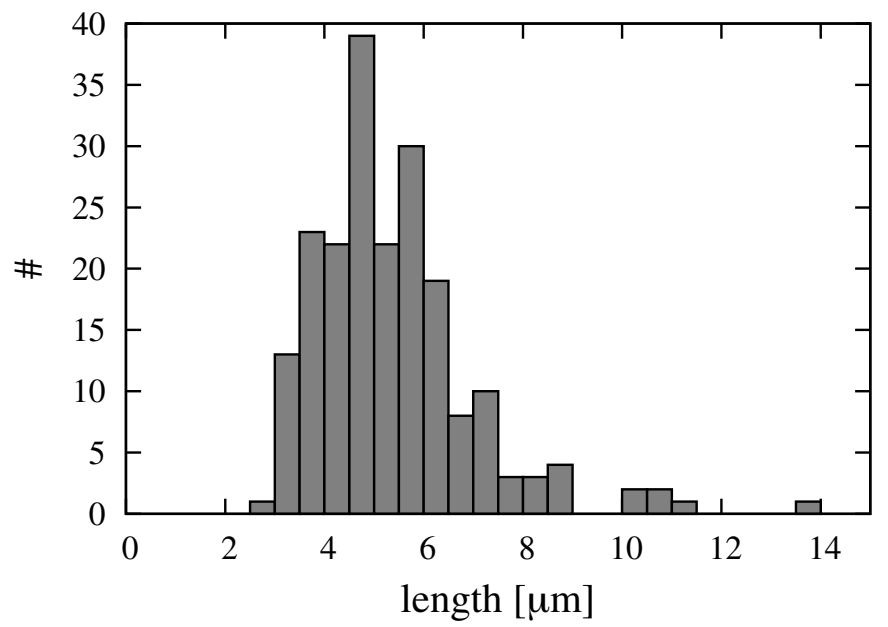

FIGURE 7.18 - Number of magnetotactic bacteria (MTB) as a function of the length of the MTB as measured by optical microscopy.

channel is less than $15 \%$. If the spiral model approaches the wall, the drag rapidly increases. At $L / 50$, the drag increases by $60 \%$. It is tempting to translate this effect to real MTB. It should be noted however that the 3D printed models are rigid and stationary, whereas the MTB are probably more flexible and mobile. Intuitively, one might expect a lower drag.

From the bacterial dimensions, we can estimate a mean rotational drag coefficient, $f_{\mathrm{b}}$, of $67(7) \mathrm{zNs}$. Since the relation between the rotational drag and the bacterial dimensions is highly nonlinear, a Monte Carlo method was used to estimate the error and variation of $f_{\mathrm{b}}$. For these calculations, the length of the bacteria was assumed to be Gaussian distributed with parameters as indicated in table 7.1.

Due to the nonlinearity, the resulting distribution of $f_{\mathrm{b}}$ is asymmetric. So rather than the standard deviation, the 10 to $90 \%$ cut-off values of the distribution are given in table 7.2. Most of the MTB are estimated to have a drag coefficient in the range of 30 to $120 \mathrm{zNs}$.

\section{Estimate of magnetic torque}

Figure 7.21 shows typical transmission electron microscopy images (TEM) of magnetosome chains. From these images, we obtain the magnetosome count $n$, radius $r$, and distance $d$, which are listed as well in table 7.1. These values agree with those reported in the literature (Faivre et al., 2010; Pósfai et al., 2007) and lie within the range of single-domain magnets (Faivre, 2015). We have found 


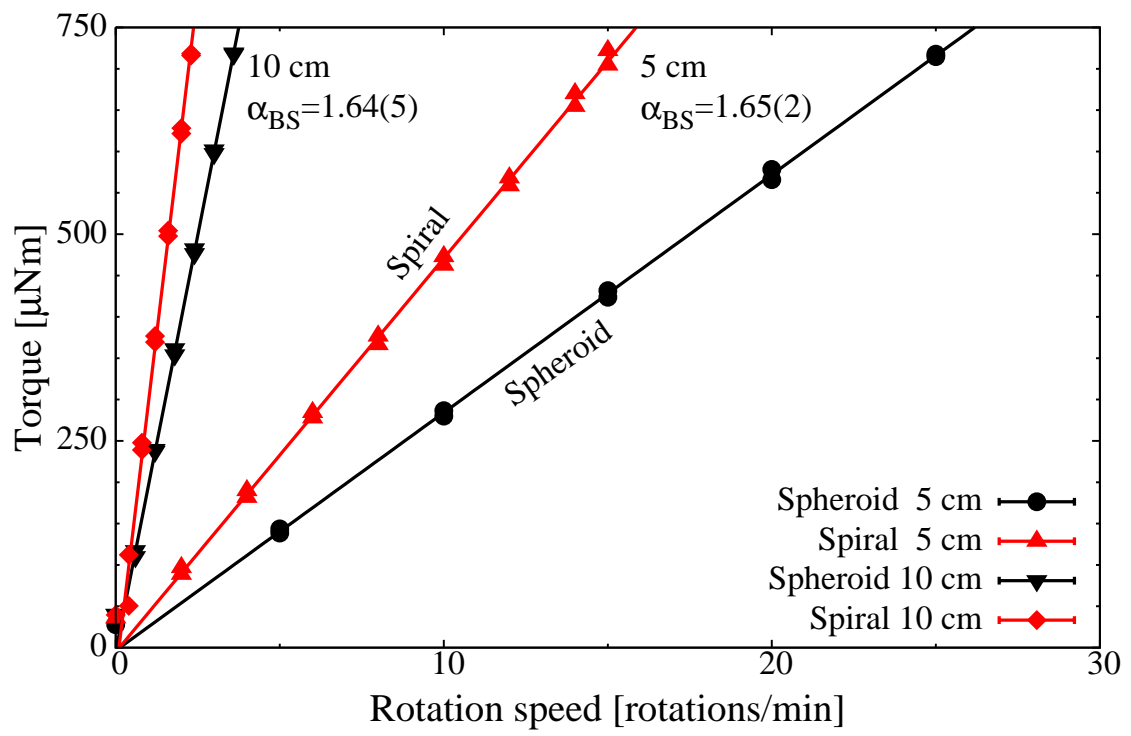

FIGURE 7.19 - Rotational drag torque versus angular rotation speed of $3 D$ printed prolate spheroids and MTB models of lengths 5 and $10 \mathrm{~cm}$. The curves are linear, indicating that the flow around the objects is laminar. Irrespective of the length, the spiral shaped MTB model has a drag that is 1.64(5) higher than a prolate spheroid of equal overall length and diameter.

TABLE 7.1 - Characteristics of magnetotactic bacteria. Length L and width W and amount $n$, diameter $d$ and spacing a of the crystals in the magnetosomes. The error indicated on the means is the standard error (standard deviation/square root of the total number of samples).

\begin{tabular}{lccccc} 
& $\begin{array}{c}L \\
{[\mu \mathrm{m}]}\end{array}$ & $\begin{array}{c}W \\
{[\mathrm{~nm}]}\end{array}$ & $n$ & $\begin{array}{c}d \\
{[\mathrm{~nm}]}\end{array}$ & $\begin{array}{c}a \\
{[\mathrm{~nm}]}\end{array}$ \\
\hline mean & $5.0(2)$ & $240(6)$ & $16(2)$ & $40(2)$ & $56(1)$ \\
stddev & 1 & 28 & 6 & 9 & 8
\end{tabular}




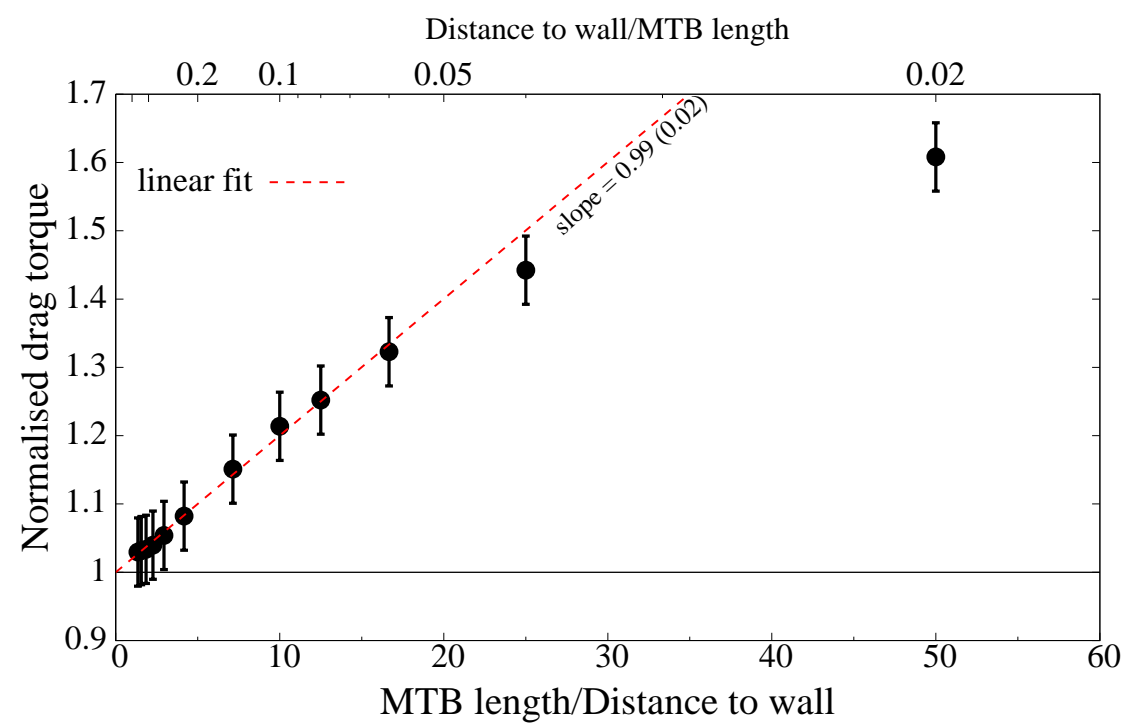

FIGURE 7.20 - Increase in rotational drag as a function of the distance between the $3 D$ printed spiral and the bottom of the container. The distance is normalized to the length of the bacteria $(5 \mathrm{~cm})$. The torque is normalized to the extrapolated value for infinite distance (displayed as "linear fit").

TABLE 7.2 - From the values of table 7.1, the drag coefficient $f_{r}$, demagnetisation factors $\Delta N$, magnetic moment $m$, maximum magnetic torque $\Gamma_{\text {max }}$, and proportionality factor $\gamma$ are estimated $(\nu / D=\gamma B)$. The input parameters are assumed to obey a Gaussian distribution with standard deviations as in table 7.1. Using a Monte Carlo method, the standard error of the calculated parameters, and the 10\%-90\% cut-offs in the distribution, are calculated.

\begin{tabular}{lcccccc} 
& $\begin{array}{c}f_{\mathrm{b}} \\
{[\mathrm{zNms}]}\end{array}$ & $\Delta N$ & $\begin{array}{c}m \\
{\left[\mathrm{fAm}^{2}\right]}\end{array}$ & $\begin{array}{c}\Gamma_{\max } \\
{[\mathrm{aNm}]}\end{array}$ & $\begin{array}{c}\gamma_{\text {theory }} \\
{[\mathrm{rad} / \mathrm{mTs}]}\end{array}$ & $\begin{array}{c}\gamma_{\exp } \\
{[\mathrm{rad} / \mathrm{mTs}]}\end{array}$ \\
\hline mean & $67(7)$ & $0.10(2)$ & $0.25(05)$ & $7(3)$ & $1.2(3)$ & $0.74(3)$ \\
$10 \%$ & 31 & 0.03 & 0.07 & 0.7 & 0.3 & \\
$90 \%$ & 124 & 0.27 & 0.57 & 41 & 3.6 &
\end{tabular}


no significant relation between the inter-magnetosome distance and the chain length, see figure 7.22 .

From these values the demagnetisation factor $\Delta N$, the magnetic moment $m$, and the maximum torque $\Gamma_{\max }$ are calculated using the model from section 7.2.1, and tabulated in table 7.2. Again, the standard deviations of the values and the $10 \%$ - and $90 \%$ cut-off values are determined from Monte Carlo simulations.

\section{Average rate of rotation}

From the drag coefficient $f_{\mathrm{r}}$ and the maximum torque $\Gamma_{\max }$, the ratio $\gamma$ between the average rate of rotation and the magnetic field strength can be obtained using equation 7.30. This value is listed as $\gamma_{\text {theory }}$ in table 7.2 , and has a convenient value of approximately $1 \mathrm{rad} / \mathrm{mTs}$. So in the earth's magnetic field $(0.04 \mathrm{mT})$, the rate of rotation of an MSR-1 is approximately $0.04 \mathrm{rad} / \mathrm{s}$. A U-turn will take at least $78 \mathrm{~s}$.

\section{Average Velocity}

The MTBs' velocity was determined from the full set of 174 analyzed bacteria trajectories. This set has a mean velocity of $49.5(7) \mu \mathrm{m} / \mathrm{s}$ with a standard deviation $\sigma$ of $8.6 \mu \mathrm{m} / \mathrm{s}$ (figure 7.23). Using the value for the average rate of rotation $\gamma$ of approximately $1 \mathrm{rad} / \mathrm{mTs}$, this speed leads to a U-turn in the earth's magnetic field of about $1 \mathrm{~mm}$ (equation 7.30).

Comparing the velocity of Magnetospirillum Gryphiswaldensen in the vicinity of an oxic-anoxic zone (OAZ), 13(1) $\mu \mathrm{m} / \mathrm{s}$ to $23(3) \mu \mathrm{m} / \mathrm{s}$ (Lefèvre et al., 2014) (orientation towards OAZ-dependent), or without, $42(4) \mu \mathrm{m} / \mathrm{s}$ (Popp et al., 2014), suggests our value of the velocity of the MTB is not restricted by an oxygen gradient. Depending on the choice of binning, one might recognise a dip in the velocity distribution. Similar dips have been found in previous research, which were attributed to different swimming modes (Reufer et al., 2014). There might as well be possible wall-effects on bacteria caused by the restricted space in the microfluidic chip (Magariyama et al., 2005).

The measured velocity during U-turns as a function of the magnetic field strength is shown in figure 7.24. The vertical error bars display the standard error of the velocity within the group. The size of the sample group is depicted above the vertical error bars. For every sample group containing less than ten bacteria, the standard deviation of the entire population was used instead. The error in the magnetic field is due to positioning error, as described in section 7.3.4.

On the scale of the graph, the deviation from the mean velocity is seemingly large, especially below $2 \mathrm{mT}$. This deviation is however not statistically significant. The reduced $\chi^{2}$ of the fit to the field-independent model is very close to unity (0.67), with a high $Q$-value of 0.77 (the probability that $\chi^{2}$ would even exceed that value by chance, see (Press et al., 1992), chapter 15 . Within the 


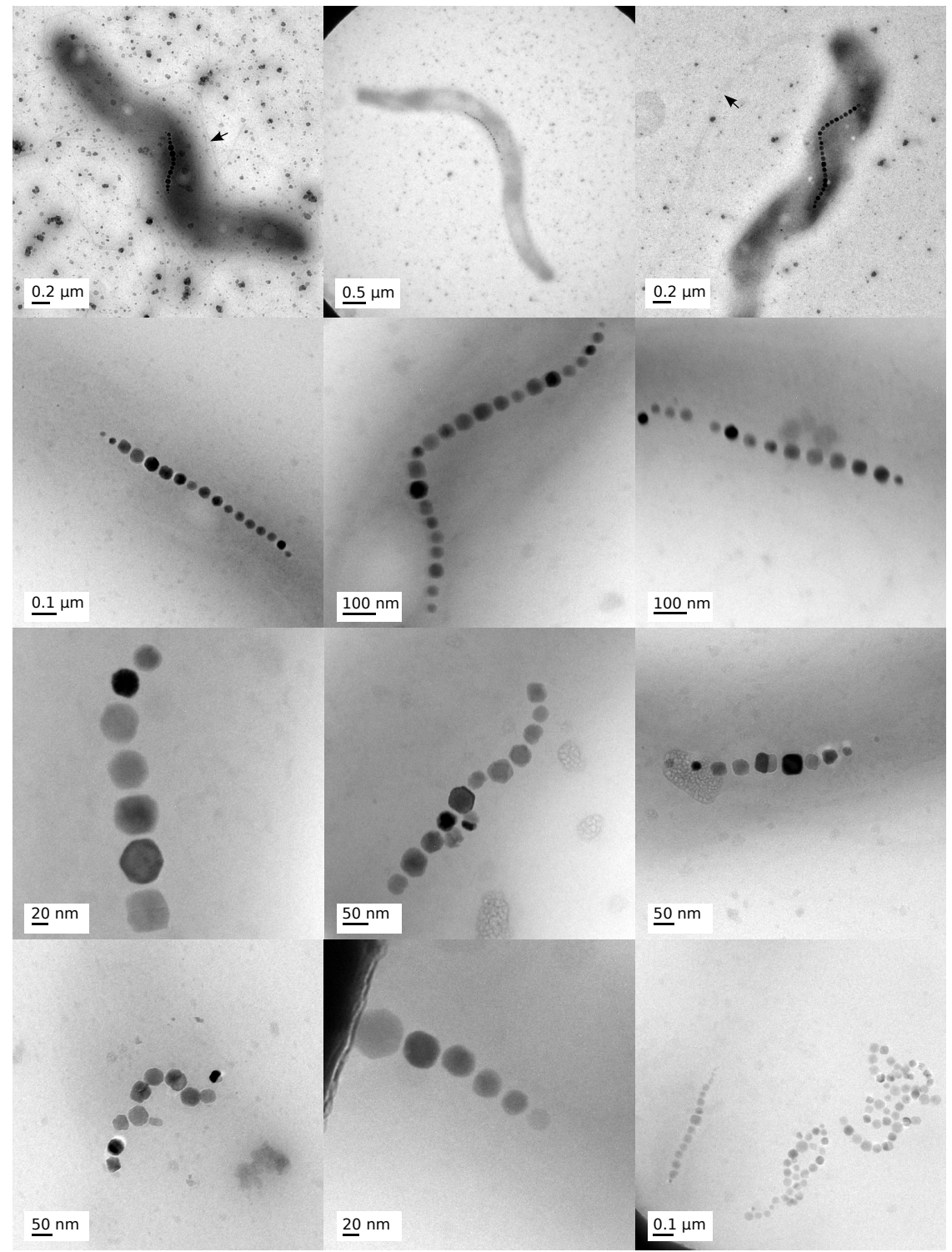

FIGURE 7.21 - Transmission electron micrographs of MSR-1, magnetosomes and chains. The top row shows typical full scale bacteria, where black arrows indicate the flagella. Compared to the second row, the third row shows shorter chains with a higher variety in size distribution of magnetic nanoparticles due to an immaturity of the chain (Uebe and Schüler, 2016). The bottom row shows irregular chains and overlapping groups of expelled chains due to the formation of aggregates, making it hard or impossible to distinguish individual chains. 


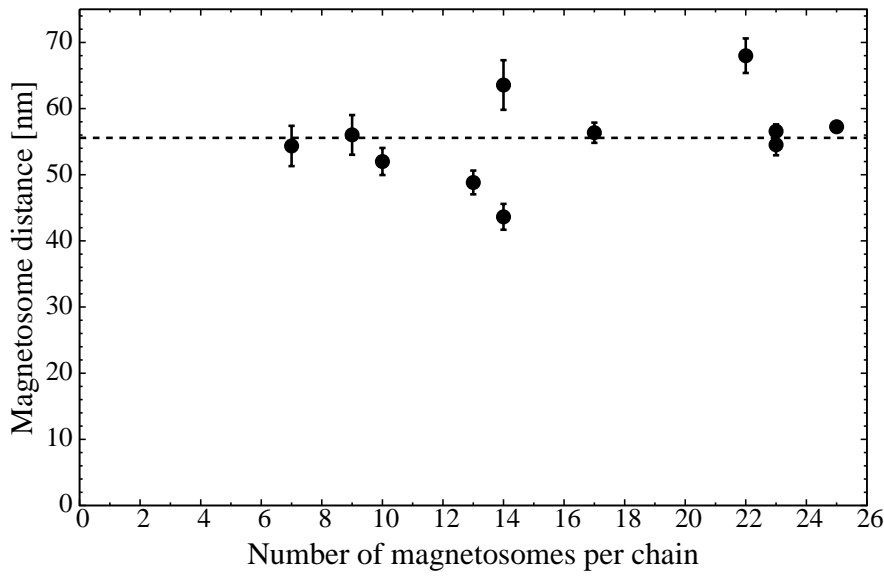

FIGURE 7.22 - Distance between magnetite particles as a function of the number of particles in the chain. The mean of the entire sample group is indicated with a dashed line at 56(1) $\mathrm{nm}$. Vertical error bars represent the standard error of each individual chain.

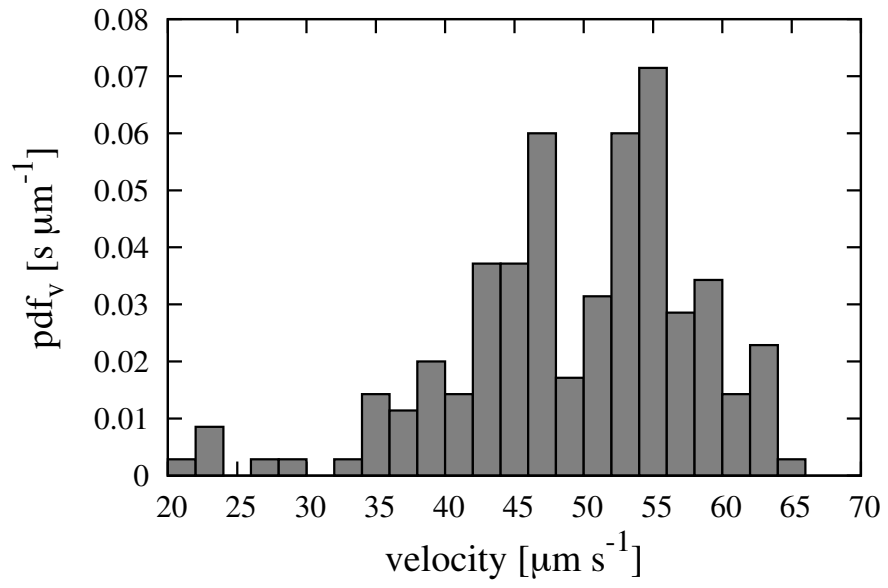

FIGURE 7.23 - Probability density function for the MTB velocity distribution for 174 observed MTB. 


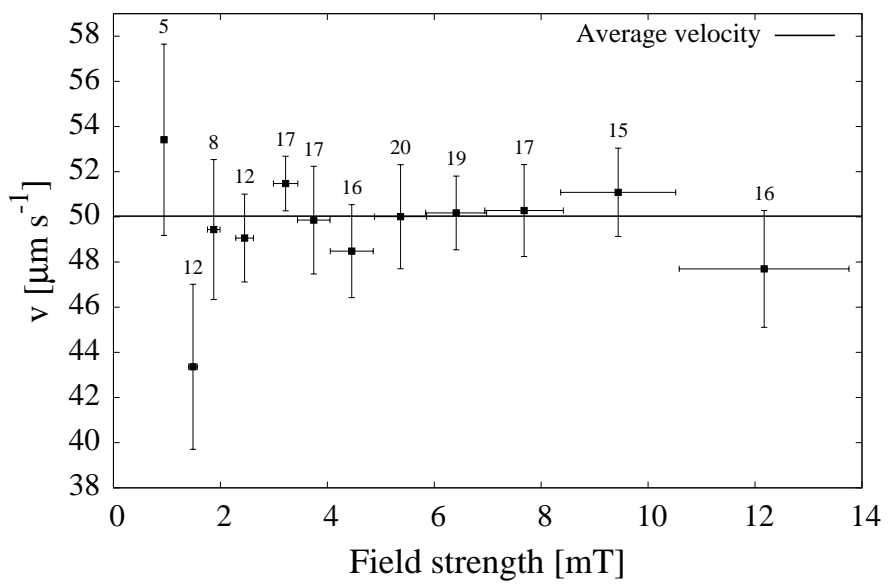

FIGURE 7.24 - Average MTB velocity as function of the applied magnetic field. The vertical error bars indicate the standard error calculated from the number of bacteria indicated above the error bar.

standard errors obtained in this measurement, and for the range of field values applied, we can conclude that the velocity of the MTB is independent of the applied magnetic field, as expected.

\subsubsection{Trajectories}

The diameter $D$ of the U-turn was measured from the trajectories as in figure 7.16. From these values and the measured velocity $v$ for each individual bacterium, the average rate of rotation $v / D$ can be calculated. Figure 7.25 shows this average rate of rotation as a function of the applied magnetic field, $B$. The error bars are defined as in figure 7.24.

The data points are fitted to the U-turn trajectory model simulations of section 7.2.2. The fit is shown as a solid black line, with the proportionality factor $\gamma_{\exp }$ equal to $0.74(03) \mathrm{rad} / \mathrm{mTs}$. The reduced $\chi^{2}$ of the fit is (2.88), and the $Q$-value (0.000 86)

Figure 7.25 shows that the observed average rate of rotation in low fields is higher than the model fit in comparison with the measurement error. We neglected the effect of the (earth's) magnetic background field. As discussed before, at this field strength, however, the average rate of rotation is on the order of $40 \mathrm{mrad} / \mathrm{s}$ and the corresponding diameter of a U-turn is on the order of $1 \mathrm{~mm}$. The background field can therefore not be the cause of any deviation at low field strengths. Tracking during the pre-processing step under low fields leads to an overlap between the trajectories, which affect the post-processing step. Due to the manual selection in the post-processing, illustrated in figure 7.16, 


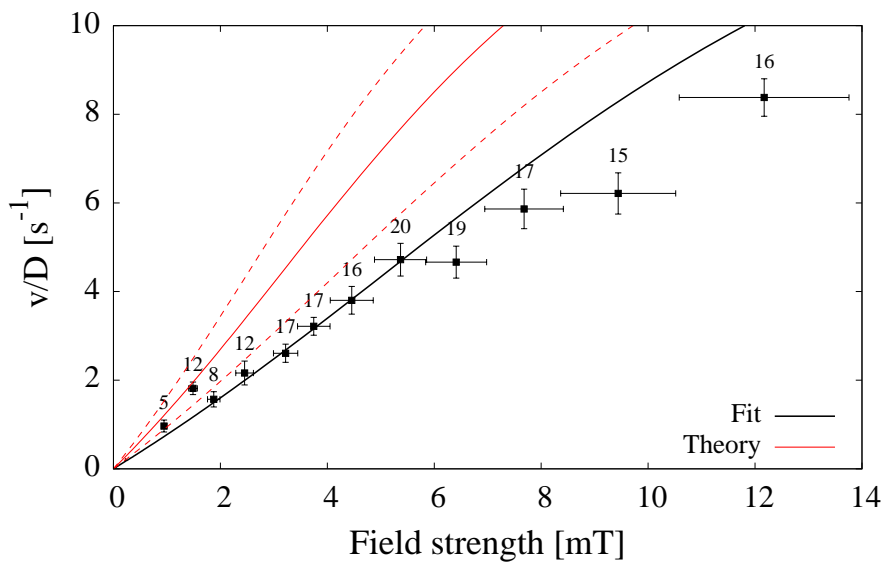

FIGURE 7.25 - The average rate of rotation, $v / D$, as a function of the applied magnetic field, B. Vertical error bars display the standard error calculated for the number of MTB denoted above the error bars. For remaining sample groups, containing less than 10 bacteria samples, the standard deviation of the entire population is used instead. The black solid line is the fit of the model to the measured data, resulting in $\gamma_{\exp }=0.74$ (3) $\mathrm{rad} / \mathrm{mTs}$. The solid red line is the model prediction, using the $\gamma_{\text {theory }}$ derived from the bacteria and magnetosome dimensions, with the dotted red lines indicating the error on the estimate (1.2(3) $\mathrm{rad} / \mathrm{mTs}$ ).

the preference for uninterrupted and often shorter trajectories may have led (for lower fields) to a selection bias to smaller curvatures. The deviation from the linear fit below $2 \mathrm{mT}$ could therefore be attributed to human bias ("cherry picking”).

If we neglect trajectories below $2 \mathrm{mT}$ for this reason, the fits improve (drastically) for both the velocity and average rate of rotation. Fitting datapoints over the range of 2 to $12 \mathrm{mT}$ (eight degrees of freedom) decreases the reduced $\chi^{2}$ of the velocity from 0.67 to 0.42 . Furthermore, the $Q$-value of 0.77 is increased to 0.91 , a slight increase in likelihood that our datapoints fall within the limits of the model.

Similarly, the reduced $\chi^{2}$ of the average rate of rotation is lowered from 2.88 to 1.03 and the $Q$-value from 0.00086 to 0.41 , a drastic change in likelihood of the fit. We therefore assume that these results validate the model with the exclusion of outliers below $2 \mathrm{mT}$.

At high fields, the observed average rate of rotation seems to be on the low side, although within the error bounds. For the high field range, the diameter of a U-turn is on the order of $5 \mu \mathrm{m}$ and reversal times are on the order of 
$100 \mathrm{~ms}$. The resolving power of our setup of $180 \mathrm{~nm} /$ pixel and time resolution of 100 frames/s are sufficient to capture these events, so cannot explain the apparent deviation. It is possible that the weakest bacteria reach the saturation torque (figure 7.4), although the effect is not expected to be very significant.

\subsection{Discussion}

Figure 7.25 shows in red the prediction of the model using the proportionality factor determined from observations of the MTB (the outer dimensions by optical microscopy and SEM, the magnetosome by TEM), $\gamma_{\text {theory }}=1.2(3) \mathrm{rad} / \mathrm{mTs}$. The predicted proportionality factor is clearly higher than measured. This is either because we overestimated the magnetic moment or underestimated the rotational drag coefficient. The latter seems more likely. In the the first place, we neglected the influence of the flagella. A coarse estimate using a rigid cylinder model for the flagellum shows that a flagellum could indeed cause this type of increase in drag. Since we lack information on the flexibility of the flagellum, we cannot quantify the additional drag. Secondly, we ignored the finite height of the microfluidic channel. As was shown by the macro-scale experiments, the additional drag increases rapidly if a bacterium approaches within a few hundred nanometres of the wall. Since we do not have information about the distance, again quantification is difficult.

Given the above considerations, we are confident that over the observed field range, the MTB trajectories are in fair agreement with our model.

\subsection{Conclusion}

We studied the response of the magnetotactic bacteria Magnetospirillum Gryphiswaldense to rotation of an external magnetic field $B$, ranging in amplitude from $1 \mathrm{mT}$ up to $12 \mathrm{mT}$.

Our magnetic model shows that the torque on the MTB is linear in the applied field up to $10 \mathrm{mT}$, after which the torque starts to saturate for an increasing part of the population.

Our theoretical analysis of bacterial trajectories shows that the bacteria perform a U-turn under $180^{\circ}$ rotation of the external field, but not at a constant angular velocity. The diameter, $D$, of the U-turn increases with an increase in the velocity $v$ of the bacteria. The average rate of rotation, $v / D$, for an instantaneously reversing field is linear within $2 \%$ in the applied field up to $12 \mathrm{mT}$.

If the applied field is rotated over $180^{\circ}$ in a finite time, the average rate of rotation is higher at low field values than it was for an instantaneous reversal. Given a field rotation time, an optimum field value exist at which the rate of rotation is approximately $18 \%$ higher than for instantaneous reversal. This optimum field value is inversely proportional to the field rotation time.

The rotational drag coefficient for an MTB was estimated from drag rotation experiments in a highly viscous fluid, using a macro-scale 3D printed MTB 
model. The spiral shape of the body of an MTB has a 64(5) \% higher drag than a spheroid with equal length and diameter, which has been the default model in the literature up to now. Furthermore, the added drag from the channel wall was found to be negligible for an MTB in the center between the walls (less than $10 \%$ ), but to increase rapidly when the MTB approaches to within a few hundred nanometres of one of the walls.

From microscope observations, we conclude that the MTB velocity during a U-turn is independent of the applied field. The population of MTB has a non-Gaussian distributed velocity, with an average of $49.5(7) \mu \mathrm{m} / \mathrm{s}$ and a standard deviation of $8.6 \mu \mathrm{m} / \mathrm{s}$. As predicted by our model, the average rate of rotation is linear in the external magnetic field within the measured range of 1 to $12 \mathrm{mT}$. The proportionality factor $\gamma=v / D B$ equals $0.74(3) \mathrm{rad} / \mathrm{mTs}$. The predicted theoretical value is $1.2(3) \mathrm{rad} / \mathrm{mTs}$, which is based on measurements of the parameters needed for the model, such as the size of the bacteria and their magnetosomes from optical microscopy, SEM, and TEM images. The number of parameters and their nonlinear relation with the proportionality factor causes the relatively large error in the estimate.

These findings finally prove that the generally accepted linear model for the response of MTB to external magnetic fields is correct within the errors caused by the estimation of the model parameters if the field values are below $12 \mathrm{mT}$. At higher values, torque saturation will occur.

This result is of importance to the control engineering community. The knowledge of the relation between the angular velocity and the field strength $(\gamma)$ can be used to design energy efficient control algorithms that prevent the use of excessive field strengths. Furthermore, a better understanding of the magnetic behaviour will lead to more accurate predictions of the dynamic response of MTB for potential applications in micro-surgery, as drug carriers, or for drug delivery. 


\title{
Chapter 8
}

\section{Real-time growth monitoring of Magnetospirillum Gryphiswaldense}

\begin{abstract}
We designed and built an optical-density (OD) based machine, and used it to automatically monitor the growth and magnetosome development of Magnetospirillum Gryphiswaldense (MSR-1) with high temporal resolution. The machine measures the optical density over time while cycling through magnetic field settings generated by coils placed around the sample, exploiting the orientation-dependent scattering of MSR-1. We quantified the bacteria density via OD, the relative and absolute amount of magnetic MTB via the differential OD signal at orthogonal magnetic field orientations, and their response to external fields (magneto-fluidic dynamics) via the step response of the OD. Additionally, the angle-dependent scattering of MSR-1 was measured and parametrised. The device offers automation of otherwise labour-intensive operations at a measurement rate of $10 \mathrm{mHz}$.

The work in this chapter is a team effort. My contribution includes composing the theoretical background and designing and building the hardware (3D printing, electronics, software). I performed the measurements and did the data analysis. Marc Pichel and Nuriye Korkmaz performed cultivation of magnetotactic bacteria and prepared the samples. Jordi Hendrix and Hans Keizer designed, built and programmed version two of the system.
\end{abstract}

\subsection{Introduction}

The growth of magnetotactic bacteria (MTB) has been extensively researched, and they are known to be difficult to cultivate (Heyen and Schüler, 2003; Liu et al., 2010). It has been found that the oxygen concentration is extremely important for both growth and magnetosome development. Several strains have proven to grow magnetosomes only in low-oxygen (micro-aerobic) conditions. 
At the same time, a too low and too high oxygen level prevents growth of the organisms (Heyen and Schüler, 2003; Schüler and Baeuerlein, 1998). Growth and Fe uptake of Magnetospirillium Gryphiswaldense (MSR-1) was found to be most optimal at a temperature of $28^{\circ}$ and a pH of 7 (Katzmann et al., 2013; Moisescu et al., 2011). Additional medium additives, such as sodium lactate (Liu et al., 2010), yeast extract and L-cysteine (Yang et al., 2001a) significantly impact the growth of MTB. Other groups have investigated the influence of growth medium composition on the growth and magnetosome formation. Ferric iron proved to be optimal for magnetic particle production of Magnetospirillum magnetotacticum (AMB-1) at a feeding rate of $1.9 \mu \mathrm{g} \mathrm{min}^{-1} \mathrm{~L}^{-1}$ and proved to inhibit growth at rates of $3.2 \mu \mathrm{g} \mathrm{min}^{-1} \mathrm{~L}^{-1}$ and over (Yang et al., 2001a). The magnetosome development of AMB-1 proves to be clearly dependent on the type of iron source, and the growth on medium composition (Yang et al., 2001b). The magnetosome size in magneto-ovoid strain MO-1 was shown to be proportional to the concentration of ferric quinate in the medium (Lefèvre et al., 2009).

In the literature, bacterium concentration is often measured by optical density (Katzmann et al., 2013; Li et al., 2009), dry weight analysis (Geelhoed et al., 2010; Heyen and Schüler, 2003) and using counting chambers (Yang et al., $2001 \mathrm{a}, \mathrm{b})$. The magnetosome growth is studied by transmission electron microscopy (TEM) (Ban et al., 2010; Yang et al., 2013), spectrometry (Liu et al., 2010; Moisescu et al., 2011) or by a light scattering method exploiting the fact that spirillium-type bacteria have a different optical density under different orientation, yielding parameter $C_{\text {mag }}$ (Faivre et al., 2010; Schüler et al., 1995). The literature reports several variants of this parameter (Song et al., 2014; Zhao et al., 2007). It provides a loose estimate on the average amount of magnetosomes per bacterium, although the method actually combines several measures; the ratio of magnetic and nonmagnetic bacteria and the magnetosome distribution over the population.

All reported methods have in common that they require manual sampling and analysis. Counting chambers and TEM is time intensive and the latter also requires expensive specialised equipment. All in all these methods are labourintensive and yield low temporal resolution.

We attempt to solve these problems by introducing an automated optical density meter which performs continuous optical density-based measurements obtaining information about growth in numbers as well as magnetosome development. The device measures the optical density as a measure for growth, $C_{\text {mag }}$ as a measure for the ratio of magnetic over nonmagnetic bacteria, $D_{\text {mag }}$ as a measure for the absolute amount of magnetic bacteria, and $\gamma$ as a measure for the response of MTB to magnetic fields, indirectly a measure for the average magnetic dipole moment. 


\subsection{Theory}

With increasing concentration of bacteria, the absorption or optical density (OD) of the sample will increase. We define the OD as:

$$
O D=\log _{10}\left(\frac{I_{0}}{I_{\mathrm{S}}}\right),
$$

where $I_{0}$ is the unobstructed light intensity signal and $I_{\mathrm{S}}$ is the signal strength after absorption. MSR-1 absorb more light when aligned to the light beam $\left(O D_{\|}\right)$ compared to orthogonal positioning $\left(O D_{\perp}\right)$. This property allows us to measure the $C_{\text {mag }}$, the standard for qualitatively determining the ratio of magnetic and nonmagnetic bacteria in a sample (Schüler et al., 1995):

$$
C_{\text {mag }}=\frac{O D_{\|}}{O D_{\perp}} .
$$

This method provides a ratio only, and does not provide a measure for the absolute amount of magnetic bacteria. For completeness we propose a simple differential measure which, in contrast, can:

$$
D_{\text {mag }}=O D_{\|}-O D_{\perp} .
$$

The transition between the orientation extrema can be modelled with an angle-dependent absorption factor $g(\theta)$ :

$$
O D(\theta)=O D_{\perp}+g(\theta)\left(O D_{\|}-O D_{\perp}\right) .
$$

The response of bacteria to external fields by means of $U$-turns and rotations has been modelled and used to determine their properties (Pichel et al., 2018; Esquivel and Lins de Barros, 1986; Zahn et al., 2017). Alignment of a bacterium to an external magnetic field with angle $\theta(t)$ can be described by the following differential equation:

$$
f \frac{\partial \theta(t)}{\partial t}+m B \sin (\theta(t))=0,
$$

where $f\left[\mathrm{~N} \mathrm{~m} \mathrm{~s}\right.$ ] represents the drag coefficient, $m\left[\mathrm{~A} \mathrm{~m}^{2}\right]$ the magnetic dipole moment of the bacterium, and $B[\mathrm{~T}]$ the magnetic field strength. Initially we assume the bacterium to be orthogonal to the magnetic field $\theta(0)=\pi / 2$. Solving for $\theta$ yields:

$$
\theta(t)=2 \cot ^{-1}\left(e^{\frac{m B}{f} t}\right) .
$$

The angle can be indirectly estimated by the measured optical density as described by equation 8.4. The settling time of this transition period is characterised by time constant $\tau=f / m B$. As in Pichel et al. (2018), we characterise the response of MTB to magnetic fields by $\gamma\left[\mathrm{rad} \mathrm{s}^{-1} \mathrm{~T}^{-1}\right]$ :

$$
\gamma=\frac{m}{\pi f}=\frac{1}{\pi \tau B} .
$$


So far we have left out rotational Brownian motion as a disturbing influence on the orientation of bacteria. There is an interplay between temperaturedriven rotational diffusion which prevent full alignment of MTB with the external field. The angular distribution of MTB $b(\theta)$ in a static magnetic field is fully determined by the ratio of magnetic and thermal energy according to Maxwell-Boltzmann statistics (Bryant et al., 2007):

$$
\begin{aligned}
b(\theta) & =\frac{\int_{0}^{2 \pi} e^{\frac{m B}{k T} \cos \theta} \sin (\theta) d \phi}{\int e^{\frac{m B}{k T} \cos \theta} d \Omega} \\
& =\frac{1}{2} \frac{m B}{k T} \frac{\sin (\theta)}{\sinh \left(\frac{m B}{k T}\right)} e^{\frac{m B}{k T} \cos \theta}, r
\end{aligned}
$$

where $k\left[\mathrm{~J} \mathrm{~K}^{-1}\right]$ is the Boltzmann constant and and $T[\mathrm{~K}]$ the temperature. The average angle can be obtained via the integral $\int_{0}^{\pi} b(\theta) \theta d \theta$. As it is hard to solve analytically a numerical approach has been taken. Figure 8.1 shows the angular distribution of MTB as per equation 8.8 for various magnetic field strengths, assuming a dipole moment of $0.25 \mathrm{fA} \mathrm{m}^{2}$ as reported in our earlier work (Pichel et al., 2018) and room temperature (300 K). Not surprisingly, the bacteria distribution becomes more narrow and more concentrated as the field amplitude increases. Additionally, perfect alignment of MTB with the field is very rare, as for very small angles there is no magnetic torque and Brownian motion is dominant. In the right figure we see the average angle as a function of the energy ratio. In absence of a field the MTB have random orientation $\left(\theta_{\text {avg }}=90^{\circ}\right)$. For very strong fields, the average angle will saturate to a nonzero asymptote due to saturation of the magnetic torque appearing between 10 to $100 \mathrm{mT}$ (Pichel et al., 2018). The earth magnetic field, approximately $50 \mu \mathrm{T}$, is just strong enough to direct only half of all bacteria within $70^{\circ}$ of the field. However, the perturbations of rotational diffusion will integrate over time, resulting in an average orientation in the direction of the field.

\subsection{Materials and methods}

Figure 8.2 gives an overview of the OD meter. A green LED (Cree LC503FPG130P-A3, dominant wavelength at $527 \mathrm{~nm}$ ) transmits light through the sample in a sealed cuvette. Photo diodes measure the intensity of the light source both before and after passing the sample. A window of $1 \times 5 \mathrm{~mm}$ blocks out light that would reach the photo diode indirectly. Two sets of Helmholtz coils are placed around the sample to generate a magnetic field at any angle with respect to the light beam. The measured current of the photo diode is converted to a voltage and amplified (LT1880 opamp), after which it is digitized at $20 \mathrm{~Hz}$ with 16-bit precision, yielding a resolution of $31 \mu \mathrm{V}$ (ADS1115 analog-to-digital converter). Both coil actuation and data acquisition was timed using Arduino hardware.

The electronic noise was characterised by measuring the response of an empty cuvette for $13 \mathrm{~min}$. After correcting the measured signal by the intensity 

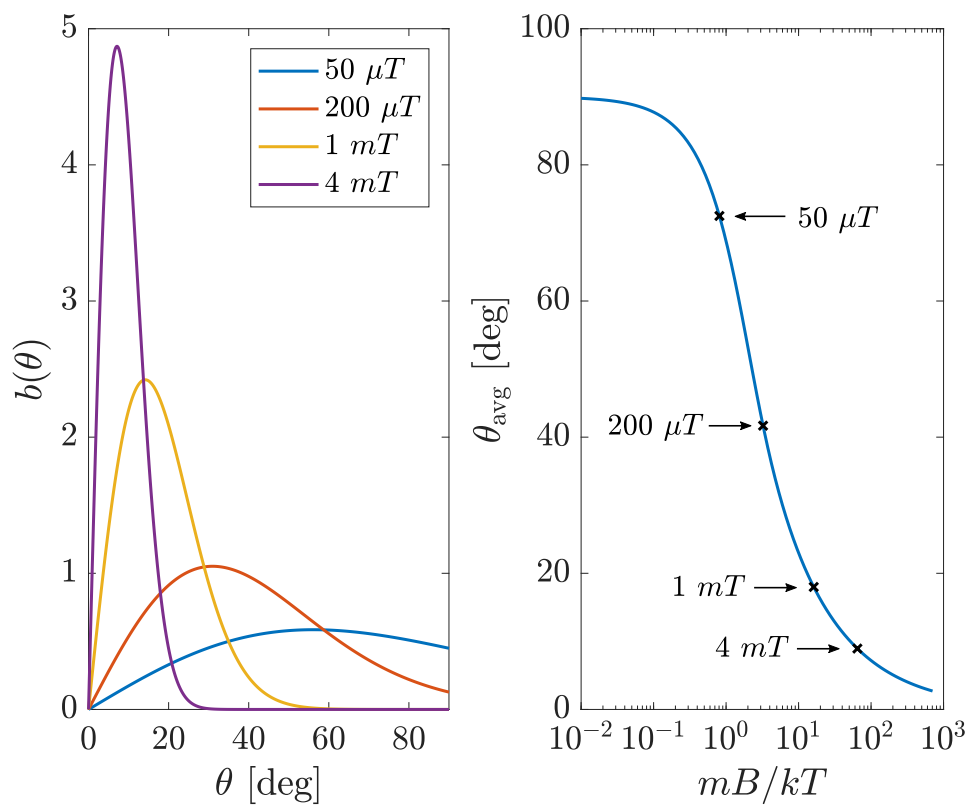

FIGURE 8.1 - Left: The angular distribution of Magnetotactic Bacteria (MTB) in a static magnetic field due to angular Brownian diffusion, assuming a reported value for the magnetic dipole moment $\left(0.25 \mathrm{fA} \mathrm{m}^{2}\right)$ (Pichel et al., 2018) and room temperature ( $300 \mathrm{~K})$. Right: the average angle of $M T B$ as a function of the ratio of magnetic and thermal energy, along with values corresponding to the left graph.

of the light source (using the signal from the reference diode), the noise is characterised by a standard deviation of $30(1) \mu \mathrm{V}$, yielding a signal-to-noise ratio of $85 \mathrm{~dB}$.

The magnetic fields generated by the coils were measured by a Metrolab THM1176 three-axis hall magnetometer and corrected carefully for misalignment with respect to the orientation of the coils.

\subsubsection{Angle dependency of OD}

The angle-dependent scatter factor was determined by measuring the OD while increasing the angle of the magnetic field with steps of approximately $2^{\circ}$. The magnetic field was at all times over $1.5 \mathrm{mT}$. As the OD of the sample is continuously fluctuating due to activity and sedimentation within the cuvette, 37 sweeps were carried out. The resulting curves were normalised to a range of 0 to 1 and averaged. 


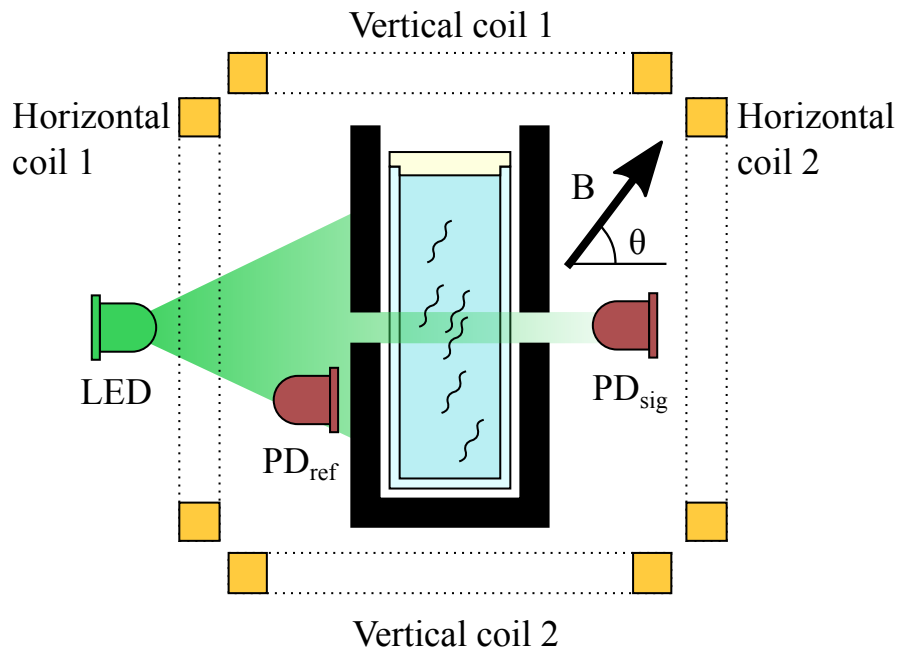

FIGURE 8.2 - The OD meter transmits a light beam through a sealed sample of $M T B$. The concentration of bacteria and their alignment determines the extent to which the light is absorbed or scattered. Two sets of coils around the sample generate a magnetic field of desired strength and angle. Photo diodes measure the light intensity both before ( $\left.P D_{\mathrm{ref}}\right)$ and after the sample ( $\left.P D_{\mathrm{sig}}\right)$.

\subsubsection{Cultivation}

Liquid cultures of Magnetospirillum Gryphiswaldense, strain MSR-1, were grown unagitated at room temperature (21 Celcius in closed $2 \mathrm{~mL}$ (Eppendorf) centrifugation tubes for 4 to 10 days. Modified spirillum growth medium (ATCC 1653) with an oxygen concentration of 1 to $3 \%$ was prepared with an adjusted $\mathrm{pH}$ of 6.8. The cultures had an average optical density at the end of cultivation of $0.3($ at $550 \mathrm{~nm})$ in the presence of a magnetic field $(5 \mathrm{mT})$ oriented parallel to the light beam. Sampling for experiments was done using chemically sterilized cuvettes (VWR). After being filled with MSR-1 in liquid solution to leave no head space, the cuvettes were sealed with four layers of UV sterilized parafilm to allow minor air (oxygen) transfer through the membrane.

\subsubsection{Growth experiment}

Several prepared samples were grown in the OD meter for a period of five days. The magnetic field was set to loop through cycles of $100 \mathrm{~s}$ using the following settings:

- Setting 1: $20 \mathrm{~s}$ a vertical field of $1.0 \mathrm{mT}$

- Setting 2: $20 \mathrm{~s}$ a horizontal field of $2.9 \mathrm{mT}$ 
- Setting 3: $60 \mathrm{~s}$ a vertical field of $100 \mu \mathrm{T}$

The transition from 1 to 2 uses relative strong fields, guaranteeing reliable estimations of $C_{\text {mag. }}$. The transition from 2 to 3 guarantees a relative large time constant, helpful for accurately estimating $\tau$. These settings allow the OD meter to measure the sample parameters with $10 \mathrm{mHz}$.

Figure 8.3 shows the measured response of a sample of magnetotactic bacteria to a cycle. It visualizes a short settling time of the OD when a strong magnetic field is used (around $25 \mathrm{~s}$ ). The longer settling time in case of the second transition (around $45 \mathrm{~s}$ ) is characterised by $\tau$. The transition at $5 \mathrm{~s}$ demonstrates the effect of the magnetic field strength on the angular distribution of MTB as visualized in figure 8.1 .

The parameters $O D_{\|}$and $O D_{\perp}$ are estimated from the last $9 \mathrm{~s}$ of settings 1 and 2, after the signal has settled. The time constant is estimated from fitting the model of equations 8.4 and 8.6 using the sum of squared errors criterion. It uses a fitting window of $45 \mathrm{~s}$. Before fitting, data is lowpass filtered using a rectangular filter in the frequency domain with a cutoff frequency of $9 \mathrm{mHz}$, in order to minimize influence of low-frequency fluctuations in the sample. Finally, $\gamma$ is determined using equation 8.7.

\subsection{Results}

\subsubsection{Angle dependency of OD}

Figure 8.4 shows the angle-dependent scatter factor $g(\theta)$. It is highest when bacteria are aligned with the light beam and lowest when orthogonal. Parametrising requires a function that is periodic and is symmetric around 0 and 90 degrees. Based on visual inspection, we choose zero derivatives at 0 and 90 degrees. Phenomenologically this can be modelled by a cosine function using only the even terms (the value in brackets represents the $95 \%$ confidence interval):

$$
\begin{aligned}
g(\theta) & =\quad \sum_{n=0}^{2} a_{n} \cos (2 n \theta) \\
\mathbf{a} & =\left[\begin{array}{lll}
0.559(2) & 0.495(3) & -0.057(3)
\end{array}\right]
\end{aligned}
$$

\subsubsection{Growth curve}

Figure 8.5 shows the measured parameters of a sample of MTB over time; the OD, $D_{\text {mag, }} D_{\text {mag }}$ and $\gamma$. The growth is characterised by a lag phase (L), exponential phase (E), stationary phase (S), magnetic growth phase (M) and an undefined phase $(\mathrm{X})$. The magnetic growth phase is defined independently of OD, and overlaps with other phases (in this case the stationary phase). The sample initially is characterised by a low OD in the lag phase, but by a relative high $C_{\text {mag. }}$ Although the fraction of magnetic bacteria is high, the absolute number is low, characterised by a low $D_{\text {mag. }}$. As the sample enters the exponential 


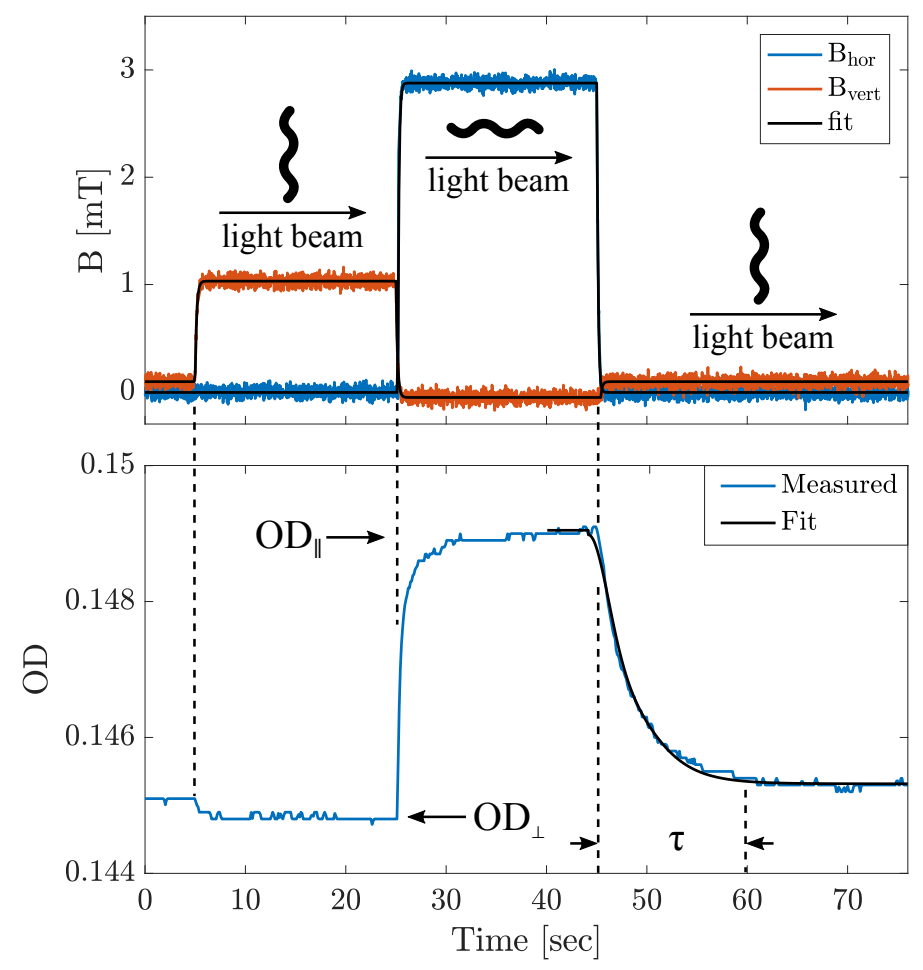

FIGURE 8.3 - A cycle of the magnetic field in horizontal and vertical direction (top) and the resulting measured response of MTB (bottom). The magnetic field settings direct the MTB from orthogonal orientation with respect to the light beam to parallel orientation and back again. $C_{\mathrm{mag}}$ and $D_{\mathrm{mag}}$, proportional to the ratio of magnetic bacteria and their absolute numbers, are calculated from the absorption in parallel $\left(O D_{\|}\right)$and orthogonal $\left(O D_{\perp}\right)$ orientations. The time constant $\tau$ quantifies the alignment speed of the MTB to the field transition.

growth phase, the $C_{\text {mag }}$ plummets while $D_{\text {mag }}$ is unaffected. This indicates that the amount of bacteria increases (as reflected in the OD) but the amount of magnetic bacteria does not. Subsequently in the stationary phase, the OD, $C_{\mathrm{mag}}$ and $D_{\text {mag }}$ remain relative stable. Only after approximately two days, in the magnetic growth phase, the amount of magnetic bacteria increases, reflected in both the $C_{\text {mag }}$ and $D_{\text {mag }}$, while the OD is not significantly affected. After approximately 3.3 days the amount of magnetic bacteria decreases, while the OD keeps increasing. This phenomenon could be caused by a contamination, or by migration of bacteria within the cuvette.

The measured $\gamma$ starts off relative low, and starts increasing once in the magnetic growth phase. Surprisingly, it continues to increase when the amount of 


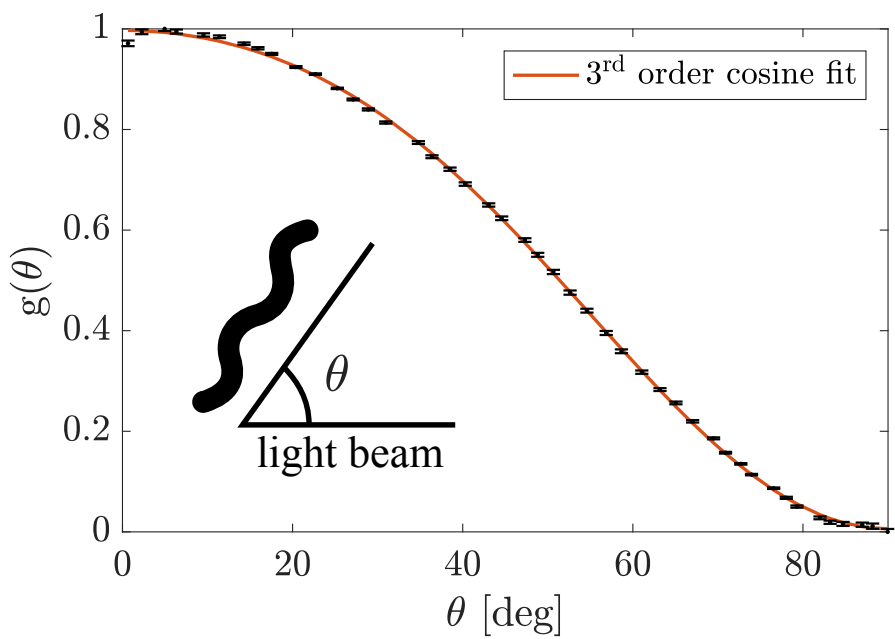

FIGURE 8.4 - The optical density of MTB is dependent on orientation; it is highest when bacteria are aligned with the light beam and lowest when they are aligned orthogonal. The angle-dependent scatter factor $g(\theta)$ shows a nonlinear dependence on orientation (see equation 8.9). Error bars represent the standard error.

magnetic bacteria declines. The average form factor of bacteria and medium viscosity is unlikely to change over time. Therefore, the continuing growth can be explained by an increased average magnetic dipole moment, or a higher average amount of iron oxide crystals per bacterium. It is not possible to determine $\gamma$ accurately before the start of the magnetic growth phase because the amplitude of the alternating OD signal is too low, as depicted in figure 8.3.

The growth profile of MTB is very sensitive to repeated experiments under slightly different conditions. Additional growth curves can be observed in Appendix B.

\subsection{Discussion}

\subsubsection{Repeatability and representability}

The observed results provide a clear insight in the growth pattern of MTB with unparalleled temporal resolution. It is unknown, however, to which extent the measured parameters are representative for the entire population residing in the cuvette. Because of the selective size of the photo diode the observation area is restricted to a small window. Only the response of bacteria in that window is measured. An uneven, time-dependent distribution of MTB in the cuvette will result in a non-representative measurement. Additionally, it is possible an 
oxygen gradient forms in the sample, contributing to a band formation that may enter and leave the observation area and enhances the uneven distribution of MTB. Indeed, the OD is fluctuating over time after the stationary phase has been reached (figure 8.5).

\subsubsection{Response of MTB to magnetic fields}

Estimation of $\gamma$ is based on a model of a single bacterium. The measured OD, however, is a bulk parameter; it is generated by bacteria that are responding with various degrees of speed to a changing magnetic field direction. The singlebacterium model is fitted to a signal consisting from both slow and fast aligning bacteria, which introduces an error. A second error results from the fact that not all bacteria are perfectly aligned with the magnetic field, but fluctuate around a mean orientation. This effect becomes stronger for bacteria with a low magnetic dipole moment, or for magnetic fields with low amplitude. This phenomenon explains why there is a change in OD from setting 3 to 1 ; in both cases the fields are directed vertically, but are an order of magnitude apart in field strength.

An additional error in estimating $\gamma$ results from samples with a significant amount of fluctuation in the measured OD characterised by medium frequencies. These fluctuations are random and cannot be modelled, but do have a significant impact on the goodness of fit.

The values for $\gamma$ range from 0.44 to $1.41 \mathrm{rad} \mathrm{mT}^{-1}$. This agrees with results we obtained in previous work, where we investigated individual bacteria (Pichel et al., 2018). We reported values of $0.74(3) \mathrm{rad} \mathrm{mT}^{-1}$ (determined via bacteria trajectories in microscope observations) and of $1.2(3) \mathrm{rad} \mathrm{mT}^{-1}$ (determined via TEM microscopy of magnetosomes and macroscopic drag models).

\subsection{Conclusions}

We have constructed an optical density-based (OD) meter which can automatically and continuously measure properties of magnetotactic bacteria (MTB). These properties include the optical density (OD), a measure for the relative amount of magnetic over non-magnetic bacteria $\left(C_{\text {mag }}\right)$, a measure for the absolute amount of magnetic bacteria $\left(D_{\mathrm{mag}}\right)$ and a parameter quantifying the alignment speed of bacteria to external magnetic fields $(\gamma)$.

The angle-dependent optical density of MTB was measured and parametrised. This relation proves to be a non-symmetrical S-curve; the OD is highest when bacteria are aligned with the light beam and lowest when positioned orthogonally.

We obtained growth curves of MTB with high temporal resolution $(10 \mathrm{mHz})$ over a period of 5 days. We clearly distinguished separate growth phases (lag, exponential growth, stationary, and magnetic growth). Initially the sample contained a significant amount of magnetic bacteria, characterised by a relative 
high $C_{\text {mag }}$ and low $D_{\text {mag }}$ in the lag phase. The exponential growth phase saw an increase of non-magnetic bacteria only, which reflected in a drop in $C_{\mathrm{mag}}$ and a stable $D_{\text {mag }}$. The magnetic growth phase started parallel to the stationary phase, reflected by an increase in both $C_{\text {mag }}$ and $D_{\text {mag. }}$.

The $\gamma$ has shown to slowly increase over the span of time, starting at the begin of the magnetic growth phase. As both the viscosity of the medium and the average shape factor of bacteria do not significantly change over time, this increase is proportional to the increase of the average magnetic dipole moment of MTB.

Measurements of growth curves of several samples have shown significant differences between the measured parameters. This demonstrates the need and importance of automated, real-time measurements of these parameters; for the purpose of optimally cultivating bacteria with desired properties, or for measuring the impact of environmental changes on growth each new sample needs to be carefully monitored. This machine requires no manual operations like sampling the culture for OD- and $C_{\text {mag }}$ measurements. Additionally it does not require time-consuming, expensive and specialised machinery such as TEM imaging for determining the magnetosome development.

The methods used by the OD meter are not without flaw; the results represent but a sub-population of the sample which might not be representative for the whole. Additionally, model inaccuracies and sample fluctuations occasionally result in non-reliable estimates of $\gamma$, as well as for samples characterised by low $C_{\text {mag. }}$ Yet, the method offers unparalleled temporal resolution and automated monitoring of MTB. We are convinced it will be an extremely helpful tool in culturing MTB, and possibly for other fields, such as monitoring magnetic particles in fluids. 


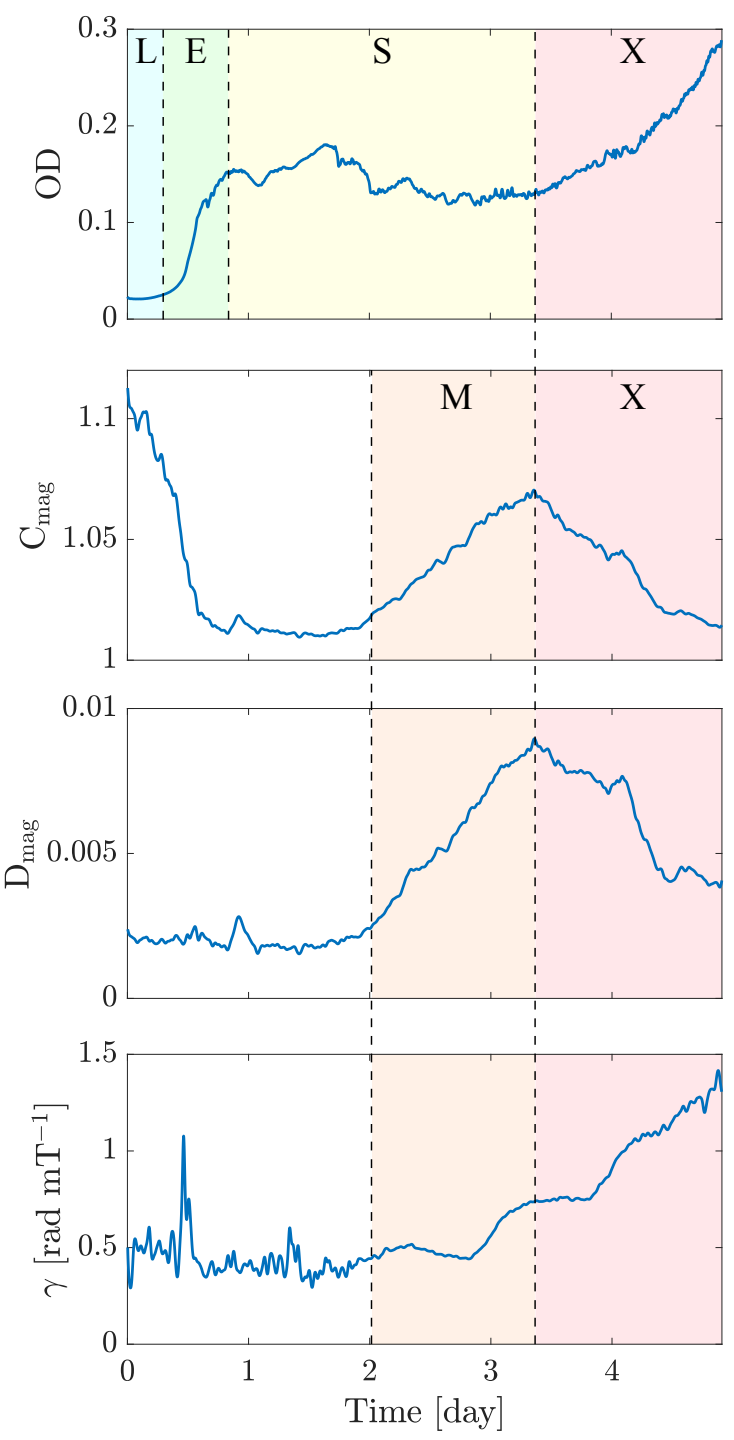

FIGURE 8.5 - The bacteria colony parameters measured over a span of five days; the optical density $(O D)$, the $C_{\mathrm{mag}}$ (quantifying the ratio of magnetic to nonmagnetic bacteria), the $D_{\text {mag }}$ (quantifying the amount of magnetic bacteria), and the $\gamma$ (quantifying how strong the bacteria respond to magnetic fields). The following phases can be identified: lag phase (L), exponential phase (E), stationary phase (S), magnetic growth phase $(M)$ and an undefined phase $(X)$. 


\section{Chapter 9}

\section{Conclusion}

This thesis was focused on answering the following research question:

WHAT CAN WE LEARN FROM OBSERVING MAGNETIC OBJECTS IN FLUIDS?

Two cases were studied; magnetotactic bacteria (MTB) and macroscopic self-assembly. These magnetic objects are seemingly extremely different; they act on different scales (micrometers versus centimetres), involve alive versus dead systems, and move and interact self-propelled versus passive. However, there are many concepts that are shared among them.

First of all, both projects deal with particles in fluids and thereby are both subject to drag force. The scale difference causes the drag profile to be different in nature, as the environments are characterised by laminar and turbulent flow. Yet the particles on macroscopic scale are modelled as if on a microscopic scale, using concepts as Brownian motion, diffusion and the Einstein relation. These concepts fit surprisingly well.

Both objects have an internal permanent magnet which, for modelling purposes, can be well approximated by magnetic dipoles. MTB have a relative weak dipole moment, are controlled via relative strong external fields and have no significant mutual interaction. In contrast, self-assembly particles have a relative strong dipole moment, are only mildly influenced by the earth magnetic field and are designed for particle-particle interaction. The magnetosome chains of MTB are composed of iron oxide nano-crystals and allow no significant structural deformation, but can change their magnetization direction when subject to a sufficiently strong external magnetic field. In comparison, the chains formed by spherical self-assembly particles are very flexible, such that they rotate while leaving their magnetization untouched.

Macroscopic analogues have shown to be helpful in providing information on their microscopic counterpart. Scaled-up objects provided a more accurate rotational drag coefficient for MTB and provided initial insights on the influence of geometry on colloidal magnetic crystal growth. 
Brownian motion is a dominant phenomenon on microscopic scale, and governs the motion on many particles. Although negligible in comparison to the self-propellant force of MTB, they still contribute significantly to the rotational diffusion. The magnetosome development of MTB is just high enough to counteract the rotational diffusion by magnetic torque. In self-assembly, we manually tune the disturbing force to be significant, but not dominant over the magnetic forces between the particles. Both systems work on a balance between an (equivalent) thermal energy and an aligning or collecting magnetic potential energy.

In both systems the theory of thermodynamics is important such that statistical mechanics can be well used to describe their states (orientation of bacteria, velocity and spacing of particles). Observation of the motion of the bacteria and macroscopic particles have helped us to complete such models by estimating parameter of either the particles themselves (dipole moment), the environment (equivalent thermal energy) or the interaction between the two (drag- and diffusion coefficient).

A majority of the results in this thesis were obtained by analysing video footage. Data was collected using conventional equipment (camera's, microscopes, computers) and image processing techniques. There is no technical reason these experiments could not have been carried out a decade ago; the novelty of this research results from modelling the motion of magnetic objects in fluids, for which detection, tracking and classification have been used as tools. Most of the code needed for these operations, as well as for further (statistical) analysis has been written by myself.

\subsection{Macroscopic self-assembly}

The first part of this thesis was focused on macroscopic self-assembly as an analogue simulation for microscopic self-assembly by investigating the motion and interaction of turbulence-driven magnetic particles.

\section{Macroscopic Brownian motion}

From trajectory analysis of single centimetre-sized spherical particles suspended in a turbulent water flow, we have observed that their velocity is MaxwellBoltzmann distributed and that they show an average squared displacement in agreement with a confined random walk. The diffusion coefficient and velocity is independent on particle size. A non-uniform particle distribution in the reactor reflects that different thermal conditions exist throughout the reactor. The interaction between two particles can be qualitatively modelled by Maxwell-Boltzmann statistics. Despite this the equivalent thermal energy determined from two-particle experiments is an order of magnitude lower than the disturbing energy determined from velocity (kinetic energy) and diffusion 
(Einstein relation). This means that the disturbing energy cannot be calibrated from single sphere experiments alone. These observations show a surprising similarity between the motion of macroscopic, turbulence-driven particles and Brownian motion.

\section{Disturbing energy}

By increasing the asymmetry of inflow of water into the reactor, the degree of turbulence can be increased, resulting in an increase in velocity, diffusion coefficient and disturbing energy. The equivalent thermal energy can be increased by a factor of six and three, determined from respectively single and two-sphere systems. Decomposing the quantities into orthogonal spatial dimensions shows that the velocity, diffusion and disturbing energy are similar for the horizontal dimensions. The vertical component shows a stronger and more eccentric dependence on flow asymmetry.

\section{Self-assembly and machine learning}

Self-assembly dynamics were studied by evaluating the structure formed by a four-sphere system over time and classifying it as "ring", "line" or "other." Analysis showed that the frequency of the structure as predicted by Maxwell-Boltzmann statistics, $63.2 \%$ and $36.8 \%$ for respectively ring and line, was significantly different than the almost $50 / 50 \%$ found by manual classification. A machine learning approach, based on a neural network trained on artificial data, came to the same conclusion. This means that a more careful study is needed towards the scaling of disturbing energy with increasingly large structures.

\section{Cluster growth}

Geometry and aspect ratio of particles are extremely important for the structures formed in magnetic self-assembly. Ellipsoidal particles form flexible, (stacked) ring-based structures. Cylindrical and cubical particles form, in contrast, primarily straight angles between particles. For cylindrical and cubical particles the aspect ratio is an important parameter to control a preference towards axial (short particles) or diametrical (long particles) connections, or to neither of them (medium particles). Aspect ratio thus is an important steric factor that influences the entropic component of the free energy. This results into respectively one-dimensional, two-dimensional (planar) and three-dimensional structures. For ellipsoidal particles this primarily has an influence on the amount of particles per ring formed. Increasing the amount of particles leads to more complex structures at the cost of a lowered structural stability. 


\section{Orbital shaker}

Turbulence is not the sole source of disturbing energy which make particles undergo a random walk. Two-dimensional orbitally shaken particles are subject to a friction which adds a stochastic displacement on top of their periodic circular motion. The velocity of this added component is nearly Rayleigh distributed, and shows Gaussian components in separate spatial dimensions. Like in the turbulence-driven reactor, the mean squared displacement is characterised by a confined random walk model. The diffusion of particles with respect to each other has a similar shape and appears to be approximately the sum of their separate mean squared displacement. Their angular displacement, however, does not obey the laws of diffusion.

\subsection{Magnetotactic bacteria}

The second part of this thesis was focused on observing both individual (microscopic) and entire colonies (macroscopic) of Magnetospirillum Gryphiswaldense, and obtaining as much of their properties from these observations such as magnetic dipole moment and rotational drag.

\section{U-turn}

Magnetotactic bacteria naturally align with external magnetic fields, and undergo a U-turn as a result of inverting the field. Their response can be modelled by simple equations, and their trajectories are very similar as to prediction based on their physiology (dimensions, geometry and magnetosomes). From studying individual bacteria it has been found that their average rate of rotation scales linear with field strength $\left(0.74(4) \mathrm{rad} \mathrm{mT}^{-1} \mathrm{~s}^{-1}\right)$, but that their velocity is independent. Furthermore, it was found that their rotational drag is in reality 64(5) \% higher than a spheroid model generally used as an approximation.

\section{Optical density meter}

The properties of entire colonies of MTB were studied during growth with high temporal resolution via optical density observations only. These include measures for their growth, the ratio of magnetic over non-magnetic bacteria, the absolute amount of bacteria and their response to magnetic fields. Under the studied conditions the magnetic growth was decoupled from the growth in numbers and started over a day later. These results demonstrate that a noninvasive method based on optical density measurements alone can quantify the most important parameters of colonies of MTB in real time. 
Appendices 

Appendix A

Velocity distribution of particles in the orbital shaker 

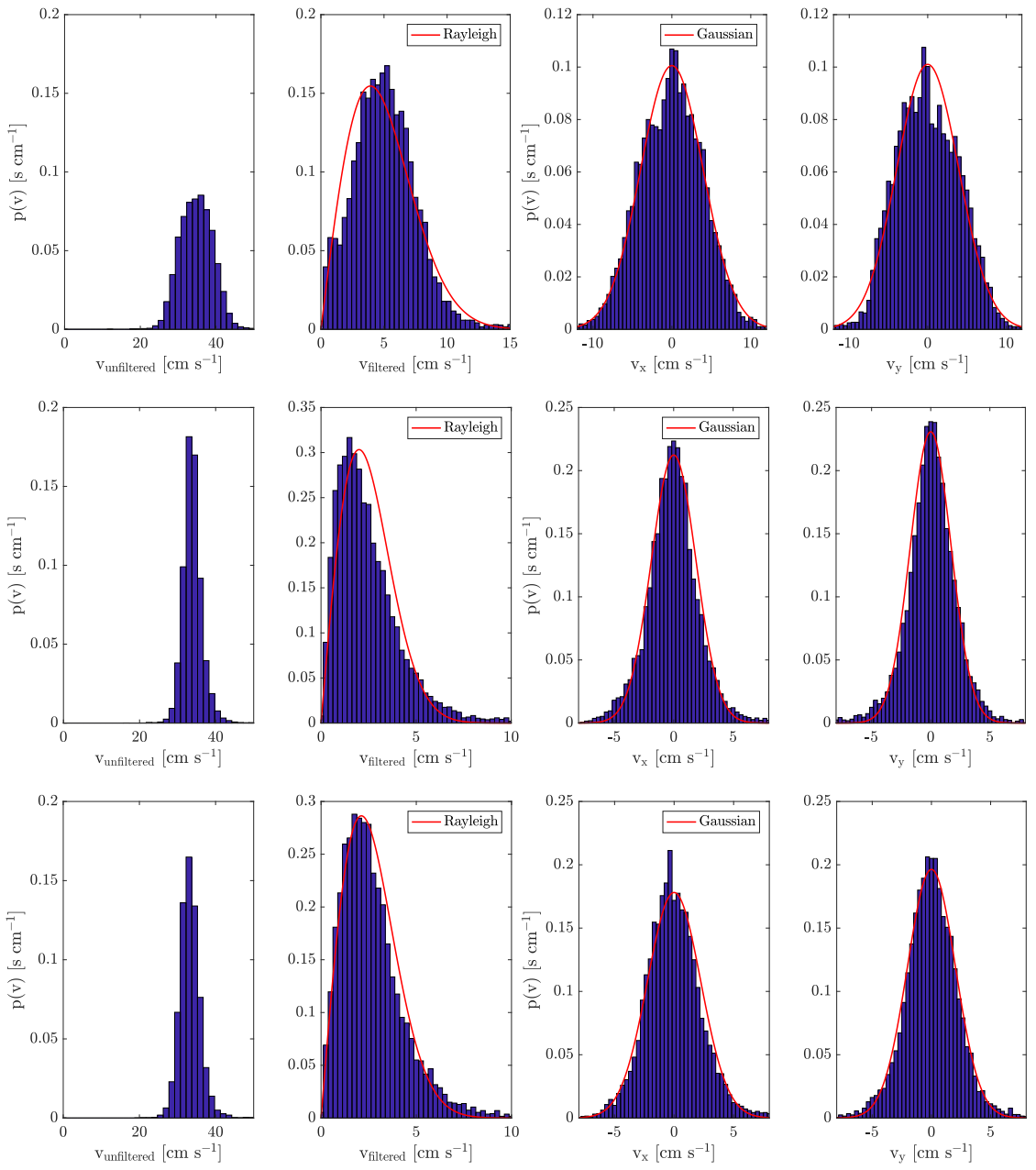

FIGURE A.1 - Velocity distributions for each particle in the 3-particle experiment in the orbital shaker. First column: unfiltered $2 D$ velocity distributions; second column: filtered $2 D$ velocity distributions and Rayleigh fitting; third and fourth column: $x$ - and y-component $1 D$ velocity distributions and Gaussian fittings. 
Appendix B

Growth curves measured in the OD meter 

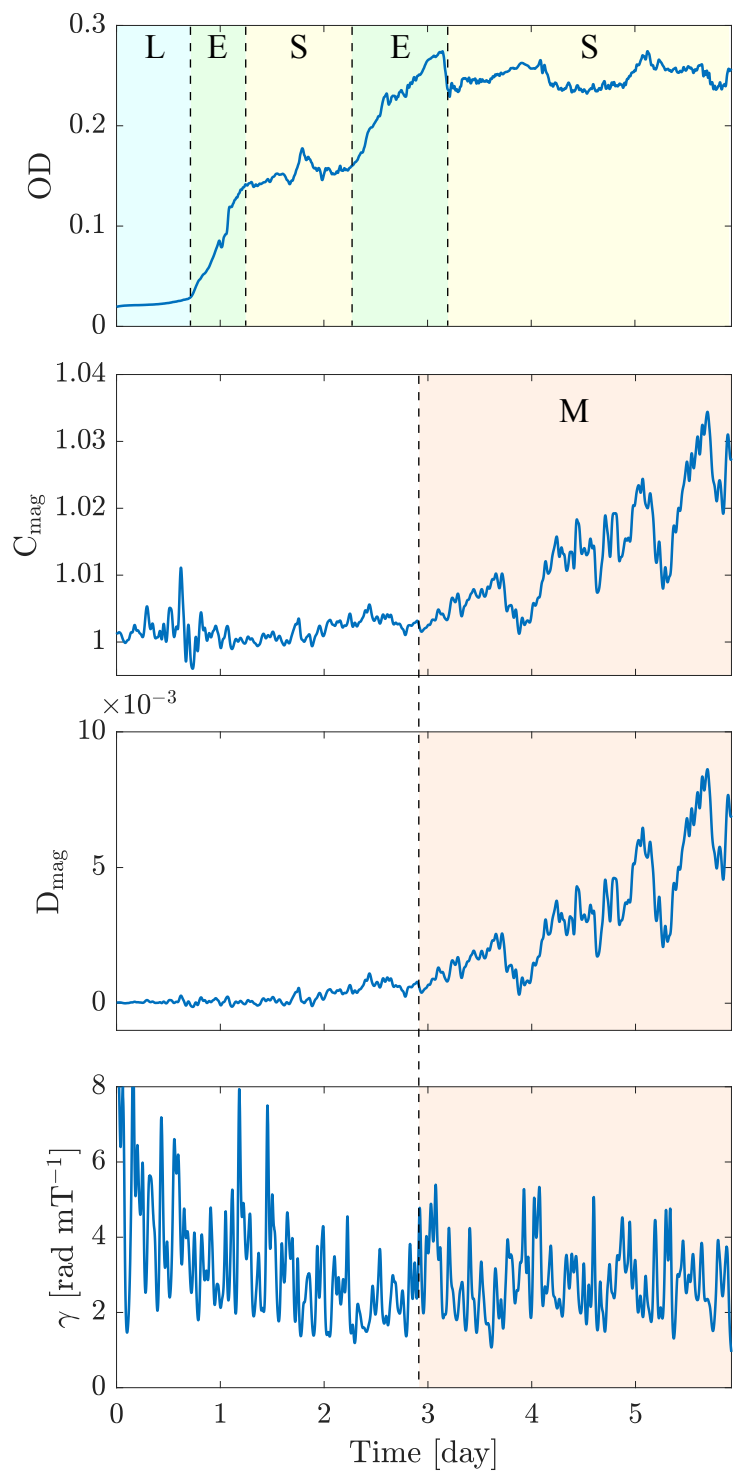

FIGURE B.1 - Measured growth parameters of a culture of Magnetospirillum Gryphiswaldense in the optical density meter. We can identify lag $(L)$, exponential growth (E) and stationary (S) phases. We observe two distinct growth phases (E); these might be actual growth phases, but the second rising slope might also be caused by migration of bacteria within the cuvette. The magnetic growth (M) is independent. There is a lot of noise in the estimation of $\gamma$. 


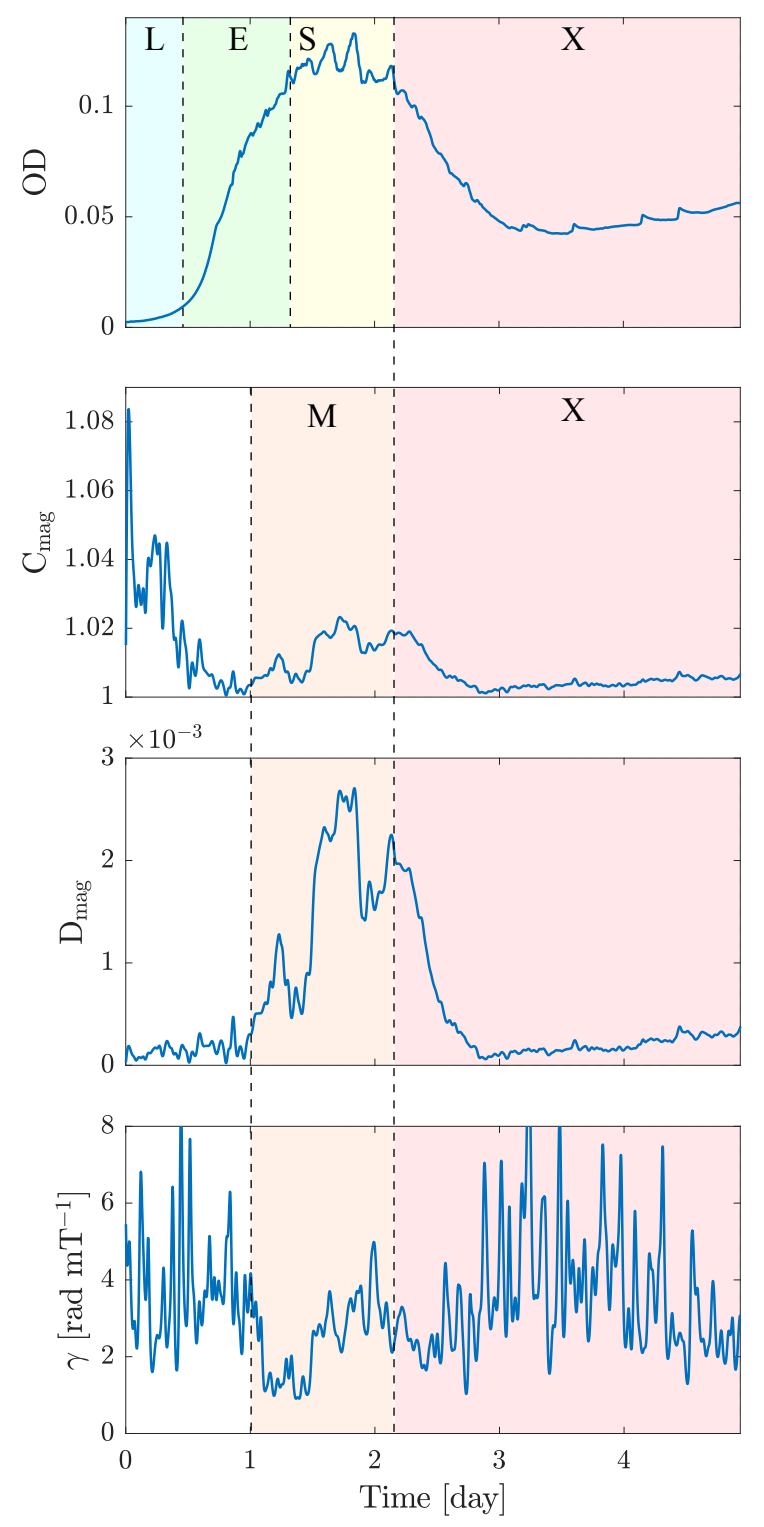

FIGURE B.2 - Measured growth parameters of a culture of Magnetospirillum Gryphiswaldense in the optical density meter. We can identify lag $(L)$, exponential growth (E) and stationary (S) phases, and an undefined phase $(X)$ in which we are not sure what happens. The latter might be caused by cell death or migration of MTB within the cuvette. The magnetic growth $(M)$ is independent. There is a lot of noise in the estimation of $\gamma$. 

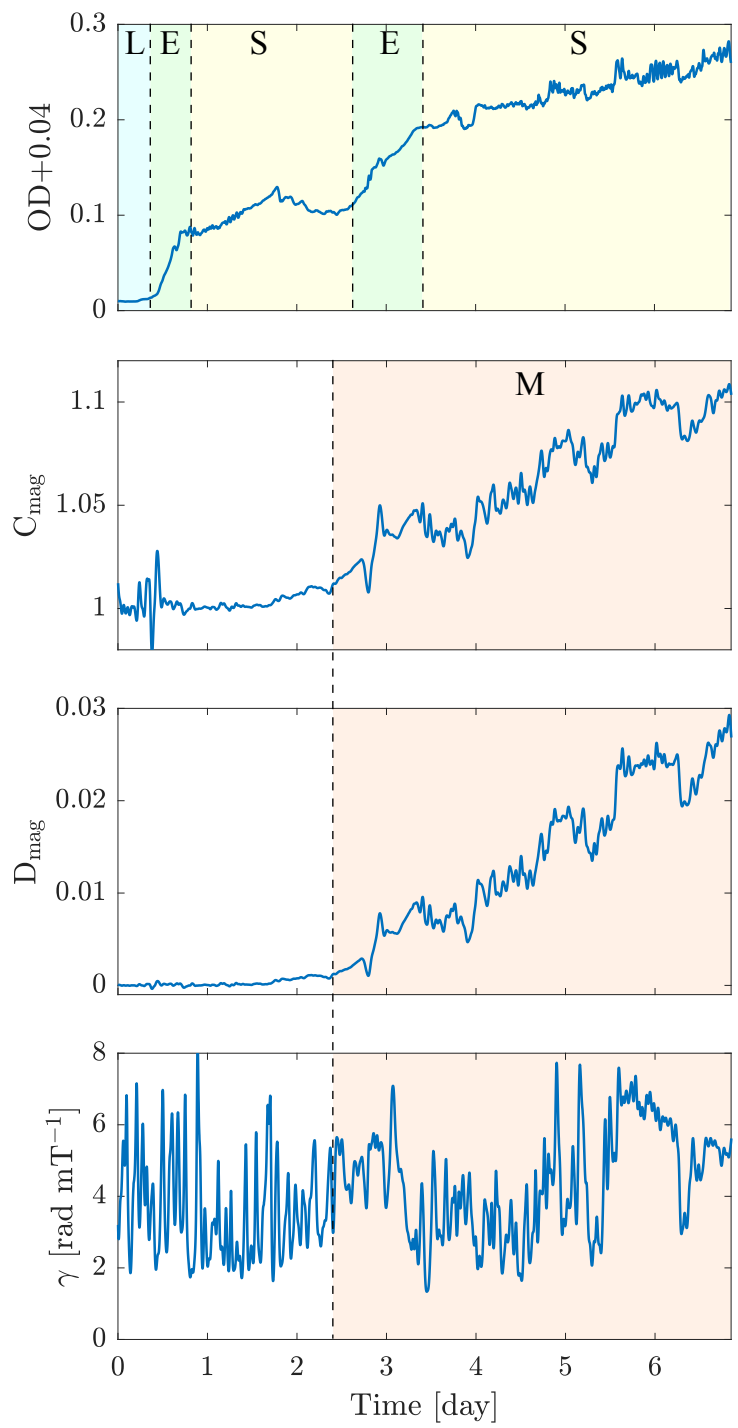

FIGURE B.3 - Measured growth parameters of a culture of Magnetospirillum Gryphiswaldense in the optical density meter. We can identify lag (L), exponential growth (E) and stationary (S) phases. We observe two distinct growth phases (E); these might be actual growth phases, but the second rising slope might also be caused by migration of bacteria within the cuvette. The magnetic growth (M) is independent. There is a lot of noise in the estimation of $\gamma$. 



\section{Bibliography}

Abayazid M, Roesthuis R J, Reilink R, Misra S, December 2013

"Integrating deflection models and image feedback for real-time flexible needle steering" IEEE transactions on robotics 29, pp. 542-553

Abbott J J, Cosentino Lagomarsino M, Zhang L, Dong L, Nelson B J, 2009

"How should microrobots swim?"

The International Journal of Robotics Research doi: 10.1177/0278364909341658

Abelmann L, Tas N, Berenschot E, Elwenspoek M, 2010

"Self-assembled three-dimensional non-volatile memories"

Micromachines 1, pp. 1-18, doi: 10.3390/mil010001

Avila K, Moxey D, De Lozar A, Avila M, Barkley D, Hof B, 2011

"The onset of turbulence in pipe flow"

Science 333, pp. 192-196, doi: 10.1126/science.1203223

Bahaj A, James P, 1993

"Characterisation of magnetotactic bacteria using image processing techniques"

IEEE Transactions on Magnetics 29, pp. 3358-3360, doi: 10.1109/20.281175

Bahaj A, James P, Moeschler F, 1996

"An alternative method for the estimation of the magnetic moment of non-spherical magnetotactic bacteria"

IEEE Transactions on Magnetics 32, pp. 5133-5135, doi: 10.1109/20.539514

Ban J, Jiang W, Li Y, Zhang Y, Li J, 2010

"Functional analysis of hydrogenases and their effects on cell growth and magnetosome synthesis in magnetospirillum gryphiswaldense"

Chinese Science Bulletin 55, pp. 1271-1277, doi: 10.1007/s11434-009-0744-8

Baumgartner J, Faivre D, 2011

"Magnetite biomineralization in bacteria"

In: W E G Muller, editor, Molecular Biomineralization, Progress in Molecular and Subcellular

Biology, volume 52, pp. 3-27, Springer Berlin

ISBN 978-3-642-21229-1, doi: 10.1007/978-3-642-21230-7_1

Bazylinski D, Frankel R, 2004

"Magnetosome formation in prokaryotes"

Nature Reviews Microbiology 2, pp. 217-230, doi: 10.1038/nrmicro842

Bellini S, 1963

On a unique behavior of freshwater bacteria,

Institute of Microbiology, University of Pavia, Italy. 
Bennewitz R, Crain J N, Kirakosian A, Lin J L, McChesney J L, Petrovykh D Y, Himpsel F J, 2002 "Atomic scale memory at a silicon surface"

Nanotechnol. 13, pp. 499-502, doi: 10.1088/0957-4484/13/4/312

Berg H C, 1993

Random Walks in Biology,

Princeton University Press

Bhalla N, Ipparthi D, Klemp E, Dorigo M, 2014

"A geometrical approach to the incompatible substructure problem in parallel self-assembly" In: International Conference on Parallel Problem Solving from Nature, pp. 751-760, Springer

van Blaaderen A, R R, Wiltzius P, 1997

“Template-directed colloidal crystallization"

Nature 385, pp. 321-323, doi: 10.1038/385321a0

van Blaaderen A, Dijkstra M, van Roij R, Imhof A, Kamp M, Kwaadgras B W, Vissers T, Liu B, Nov 2013

"Manipulating the self assembly of colloids in electric fields"

The European Physical Journal Special Topics 222, pp. 2895-2909, doi: 10.1140/epjst/e201302065-0

Boncheva M, Andreev S A, Mahadevan L, Winkleman A, Reichman D R, Prentiss M G, Whitesides S, Whitesides G M, 2005

"Magnetic self-assembly of three-dimensional surfaces from planar sheets" Proceedings of the National Academy of Sciences 102, pp. 3924-3929, doi: $10.1073 /$ pnas.0500807102

Brown P P, Lawler D F, 2003

"Sphere drag and settling velocity revisited"

J. Environ. Eng. 129, pp. 222-231, doi: 10.1061/(ASCE)0733-9372(2003)129:3(222)

Bryant H, Sergatskov D, Lovato D, Adolphi N, Larson R, Flynn E, 2007

"Magnetic needles and superparamagnetic cells"

Physics in Medicine and Biology 52, pp. 4009-4025, doi: 10.1088/0031-9155/52/14/001

Cademartiri R, Stan C A, Tran V M, Wu E, Friar L, Vulis D, Clark L W, Tricard S, Whitesides G M, 2012

"A simple two-dimensional model system to study electrostatic-self-assembly"

Soft Matter 8, pp. 9771-9791

Calegari F, Ayuso D, Trabattoni A, Belshaw L, De Camillis S, Anumula S, Frassetto F, Poletto L, Palacios A, Decleva P, Greenwood J B, Martín F, Nisoli M, 2014

"Ultrafast electron dynamics in phenylalanine initiated by attosecond pulses"

Science 346, pp. 336-339, doi: 10.1126/science.1254061

Cebers A, 2011

"Diffusion of magnetotactic bacterium in rotating magnetic field"

Journal of Magnetism and Magnetic Materials 323, pp. 279-282, doi:

10.1016/j.jmmm.2010.09.017

Chang E, Maxey M, 1994

"Unsteady flow about a sphere at low to moderate reynolds number. part 1. oscillatory motion" Journal of Fluid Mechanics 277, pp. 347-379, doi: 10.1017/S002211209400279X

Chang E, Maxey M, 1995

"Unsteady flow about a sphere at low to moderate reynolds number. part 2. accelerated motion"

Journal of Fluid Mechanics 303, pp. 133-153, doi: 10.1017/S0022112095004204 
Cooksy A, 2014

Physical Chemistry: Thermodynamics, Statistical Mechanics \& Kinetics, Pearson

Dankelman J, van den Dobbelsteen J, Breedveld P, nov. 2011

"Current technology on minimally invasive surgery and interventional techniques" In: Instrumentation Control and Automation (ICA), $20112^{\text {nd }}$ International Conference on, pp. 12-15, doi: 10.1109/ICA.2011.6130118

Darras A, Fiscina J, Pakpour M, Vandewalle N, Lumay G, 2016 "Ribbons of superparamagnetic colloids in magnetic field" The European Physical Journal E 39, p. 47, doi: 10.1140/epje/i2016-16047-0

De Lanauze D, Felfoul O, Turcot J P, Mohammadi M, Martel S, 2014

"Three-dimensional remote aggregation and steering of magnetotactic bacteria microrobots for drug delivery applications" International Journal of Robotics Research 33, pp. 359-374, doi: 10.1177/0278364913500543

Dellutri M, Pulici P, Guarnaccia D, Stoppino P, Vanalli G, Lessio T, Vassallo F, Di Stefano R, Labriola G, Tenerello A, Lo Iacono F, Campardo G, 2006

"1 Gb stacked solution of multilevel NOR flash memory packaged in a LFBGA $8 \mathrm{~mm}$ by $10 \mathrm{~mm}$ by $1.4 \mathrm{~mm}$ of thickness"

Thermal, Mechanical and Multi-Physics Simulation and Experiments in Micro-Electronics and Micro-Systems. Proceedings of EuroSimE 2006 (IEEE Cat. No.06EX1341) pp. 5 pp. -

Dennis S, Singh S, Ingham D, 1980

"Steady flow due to a rotating sphere at low and moderate reynolds numbers." Journal of Fluid Mechanics 101, pp. 257-279

Dote J, Kivelson D, 1983

"Hydrodynamic rotational friction coefficients for nonspheroidal particles" Journal of Physical Chemistry 87, pp. 3889-3893

Einstein A, 1905

"über die von der molekularkinetischen theorie der wärme geforderte bewegung von in ruhenden flüssigkeiten suspendierten teilchen"

Annals of Physics 17

Elwenspoek M, Abelmann L, Berenschot E, van Honschoten J, Jansen H, Tas N, 2010

"Self-assembly of (sub-)micron particles into supermaterials"

J. Micromech. Microeng. 20, p. 064001, doi: 10.1088/0960-1317/20/6/064001

Erb R, Son H, Samanta B, Rotello V, Yellen B, 2009

"Magnetic assembly of colloidal superstructures with multipole symmetry"

Nature 457, pp. 999-1002, doi: 10.1038/nature07766

Erglis K, Wen Q, Ose V, Zeltins A, Sharipo A, Janmey P A, Cebers A, 2007

"Dynamics of magnetotactic bacteria in a rotating magnetic field"

Biophysical Journal 93, pp. 1402-1412, doi: 10.1529/biophysj.107.107474

Esquivel D, Lins de Barros H, 1986

"Motion of magnetotactic microorganisms"

Journal of Experimental Biology VOL. 121, pp. 153-163

Faivre D, 2015

"Formation of magnetic nanoparticle chains in bacterial systems"

MRS Bulletin 40, pp. 509-515, doi: 10.1557/mrs.2015.99 
Faivre D, Fischer A, Garcia-Rubio I, Mastrogiacomo G, Gehring A, 2010

"Development of cellular magnetic dipoles in magnetotactic bacteria"

Biophysical Journal 99, pp. 1268-1273, doi: 10.1016/j.bpj.2010.05.034

Felfoul O, Mohammadi M, Taherkhani S b, De Lanauze D, Zhong Xu Y, Loghin D, Essa S d, Jancik S, Houle D, Lafleur M, Gaboury L, Tabrizian M f, Kaou N, Atkin M, Vuong T, Batist G, Beauchemin N, Radzioch D, Martel S, 2016

"Magneto-aerotactic bacteria deliver drug-containing nanoliposomes to tumour hypoxic regions"

Nature Nanotechnology 11, pp. 941-947, doi: 10.1038/nnano.2016.137

Feynman R P, Leighton R B, Sands M, 1970

The Feynman Lectures on Physics, Vol I., Addison Wesley

Frankel R, Bazylinski D, Johnson M, Taylor B, 1997

"Magneto-aerotaxis in marine coccoid bacteria"

Biophysical Journal 73, pp. 994-1000

Geelhoed J, Kleerebezem R, Sorokin D, Stams A, van Loosdrecht M, 2010

"Reduced inorganic sulfur oxidation supports autotrophic and mixotrophic growth of magnetospirillum strain $\mathrm{j} 10$ and magnetospirillum gryphiswaldense"

Environmental Microbiology 12, pp. 1031-1040, doi: 10.1111/j.1462-2920.2009.02148.x

Gillespie D T, 2007

"Stochastic simulation of chemical kinetics"

Annu. Rev. Phys. Chem. 58, pp. 35-55

Gillespie D T, Seitaridou E, 2012

Simple Brownian diffusion: an introduction to the standard theoretical models, Oxford University Press

Gorby Y, Beveridge T, Blakemore R, 1988

"Characterization of the bacterial magnetosome membrane."

Journal of Bacteriology 170, pp. 834-841

Gracias D H, Tien J, Breen T L, Hsu C, Whitesides G M, 2000

"Forming electrical networks in three dimensions by self-assembly"

Science 289, pp. 1170-1172, doi: 10.1126/science.289.5482.1170

Grant J, Jack R, Whitelam S, 2011

"Analyzing mechanisms and microscopic reversibility of self-assembly"

Journal of Chemical Physics 135, doi: 10.1063/1.3662140

Griffiths D J, 1999

Introduction to electrodynamics,

Prentice Hall, Upper Saddle River, New Jersey, 3rd edition

Gross R, Dorigo M, Sept 2008

"Self-assembly at the macroscopic scale"

Proceedings of the IEEE 96, pp. 1490-1508, doi: 10.1109/JPROC.2008.927352

Grünwald M, Tricard S, Whitesides G M, Geissler P L, 2016

"Exploiting non-equilibrium phase separation for self-assembly"

Soft Matter 12, pp. 1517-1524

Grzybowski B A, Stone H A, Whitesides G M, 2002

"Dynamics of self assembly of magnetized disks rotating at the liquid-air interface"

P. Natl. Acad. Sci. USA 99, pp. 4147-4151 
Hacohen A, Hanniel I, Nikulshin Y, Wolfus S, Abu-Horowitz A, Bachelet I, 2015

"Meshing complex macro-scale objects into self-assembling bricks"

Scientific Reports 5, doi: 10.1038/srep12257

Hageman T, Loethman P, Bienia N, Woldering L, Elwenspoek M, Manz A, Abelmann L, 42015

"Disturbing energy of a macroscopic self-assembly reactor"

In: Foundations of Nanoscience (FNANO 2015),

Hageman T A G, Löthman P A, Dirnberger M, Elwenspoek M, Manz A, Abelmann L, 2018

"Macroscopic equivalence for microscopic motion in a turbulence driven three-dimensional self-assembly reactor"

Journal of Applied Physics 123, p. 024901, doi: 10.1063/1.5007029

Hanzlik M, Winklhofer M, Petersen N, 1996

"Spatial arrangement of chains of magnetosomes in magnetotactic bacteria"

Earth and Planetary Science Letters 145, pp. 125-134

Hartley R I, Zisserman A, 2004

Multiple View Geometry in Computer Vision,

Cambridge University Press, ISBN: 0521540518, second edition

Hassan H, Pichel M, Hageman T, Abelmann L, Khalil I S M, 2016

"Influence of the magnetic field on the two-dimensional control of magnetospirillum gryphiswaldense strain msr-1"

In: Proceedings of the IEEE International Conference on Intelligent Robots and Systems (IROS), Daejeon, Korea, pp. 5119-5124,

Heyen U, Schüler D, 2003

"Growth and magnetosome formation by microaerophilic magnetospirillum strains in an oxygen-controlled fermentor"

Applied Microbiology and Biotechnology 61, pp. 536-544

Hosokawa K, Shimoyama I, Miura H, 1994

"Dynamics of self-assembling systems: Analogy with chemical kinetics"

Artif. Life 1, pp. 413-427

$\mathrm{Hu}$ B K, 2000

"Averages of static electric and magnetic fields over a spherical region: A derivation based on the mean-value theorem"

American Journal of Physics 68, pp. 1058-1060

Hu B K, 2008

"Averages of static electric and magnetic fields over a spherical region: A derivation based on the mean-value theorem"

arXiv:physics/0002021 [physics.ed-ph]

Hubert A, Schäfer R, 1998

Magnetic domains: the analysis of magnetic microstructures,

Springer-Verlag, Berlin, Heidelberg, New-York

Hwang H, Irons G, 2012

"A water model study of impinging gas jets on liquid surfaces"

Metallurgical and Materials Transactions B: Process Metallurgy and Materials Processing Science 43, pp. 302-315, doi: 10.1007/s11663-011-9613-3

Ilievski F, Mirica K A, Ellerbee A K, Whitesides G M, 2011a

"Templated self-assembly in three dimensions using magnetic levitation"

Soft Matter 7, pp. 9113-9118, doi: 10.1039/C1SM05962A 
Ilievski F, Mani M, Whitesides G, Brenner M, 2011b

"Self-assembly of magnetically interacting cubes by a turbulent fluid flow"

Phys. Rev. E Stat. Nonlinear Soft Matter Phys. 83, pp. -, doi: 10.1103/PhysRevE.83.017301

Iwase E, Shimoyama I, 2005

"Multistep sequential batch assembly of three-dimensional ferromagnetic microstructures with elastic hinges"

Journal of Microelectromechanical Systems 14, pp. 1265-1271, doi:

10.1109/JMEMS.2005.851814

Jacobs I, Bean C, 1955

"An approach to elongated fine-particle magnets"

Physical Review 100, pp. 1060-1067, doi: 10.1103/PhysRev.100.1060

van Kampen N, 1995

"The turning of magnetotactic bacteria"

Journal of Statistical Physics 80, pp. 23-33, doi: 10.1007/BF02178351

Katzmann E, Eibauer M, Lin W, Pan Y, Plitzko J, Schuler D, 2013

"Analysis of magnetosome chains in magnetotactic bacteria by magnetic measurements and automated image analysis of electron micrographs"

Applied and Environmental Microbiology 79, pp. 7755-7762, doi: 10.1128/AEM.02143-13

Khalil I S M, Pichel M P, Abelmann L, Misra S, May 2013

"Closed-loop control of magnetotactic bacteria"

The International Journal of Robotics Research 32, pp. 637-649, doi:

$10.1177 / 0278364913479412$

Kirschvink J, Walker M, Diebel C, 2001

"Magnetite-based magnetoreception"

Current Opinion in Neurobiology 11, pp. 462-467, doi: 10.1016/S0959-4388(00)00235-X

Klug A, 1983

"From Macromolecules to Biological Assemblies (Nobel Lecture)"

Angew. Chem. Int. Edit. 22, pp. 565-582

Kolmogorov A, 1941

"Local structure of turbulence in an incompressible viscous fluid at very high reynolds numbers"

C. R. Acad. Sci. URSS 30

Komeili A, Vali H, Beveridge T, Newman D, 2004

"Magnetosome vesicles are present before magnetite formation, and mama is required for their activation"

Proceedings of the National Academy of Sciences of the United States of America 101, pp. 38393844, doi: 10.1073/pnas.0400391101

Kudrolli A, 2004

"Size separation in vibrated granular matter"

Reports on progress in physics 67, p. 209

Kumar D, Nitsure N, Bhattacharya S, Ghosh S, 2015

"Granular self-organization by autotuning of friction"

Proc. Natl. Acad. Sci. USA 112, pp. 11443-11448

Landau L, Lifshitz E M, 1987

Fluid Mechanics,

PERGAMON PRESS, doi: 10.1016/B978-0-08-033933-7.50003-9 
Langevin P, 1908

"Sur la théorie du mouvement brownien"

C. R. Acad. Sci. (Paris) 146, pp. 530-533

Lash M, Fedorchak M, Little S, McCarthy J, 2015

"Fabrication and characterization of non-brownian particle-based crystals"

Langmuir 31, pp. 898-905, doi: 10.1021/la501511s

Lefèvre C, Song T, Yonnet J P, Wu L F, 2009

"Characterization of bacterial magnetotactic behaviors by using a magnetospectrophotometry assay"

Applied and Environmental Microbiology 75, pp. 3835-3841, doi: 10.1128/AEM.00165-09

Lefèvre C, Bennet M, Landau L, Vach P, Pignol D, Bazylinski D, Frankel R, Klumpp S, Faivre D, 2014 "Diversity of magneto-aerotactic behaviors and oxygen sensing mechanisms in cultured magnetotactic bacteria"

Biophysical Journal 107, pp. 527-538, doi: 10.1016/j.bpj.2014.05.043

Lemons D, Gythiel A, 1997

"Paul langevin's 1908 paper "on the theory of brownian motion" ["sur la théorie du mouvement brownien," c. r. acad. sci. (paris) 146, 530-533 (1908)]"

American Journal of Physics 65, pp. 1079-1080, doi: 10.1119/1.18725

Li J, Pan Y, Chen G, Liu Q, Tian L, Lin W, 2009

"Magnetite magnetosome and fragmental chain formation of magnetospirillum magneticum amb-1: Transmission electron microscopy and magnetic observations"

Geophysical Journal International 177, pp. 33-42, doi: 10.1111/j.1365-246X.2009.04043.x

Li T, Kheifets S, Medellin D, Raizen M G, 2010

"Measurement of the instantaneous velocity of a brownian particle"

Science 328, pp. 1673-1675, doi: 10.1126/science.1189403

Lins U, McCartney M, Farina M, Frankel R, Buseck P, 2005

"Habits of magnetosome crystals in coccoid magnetotactic bacteria"

Applied and Environmental Microbiology 71, pp. 4902-4905, doi: 10.1128/AEM.71.8.49024905.2005

Liu J, Notbohm J K, Carpick R W, Turner K T, 2010

"Method for characterizing nanoscale wear of atomic force microscope tips"

ACS Nano 4, pp. 3763-3772, doi: 10.1021/nn100246g

Love J, Urbach A, Prentiss M, Whitesides G, 2003

"Three-dimensional self-assembly of metallic rods with submicron diameters using magnetic interactions"

J. Am. Chem. Soc. 125, pp. 12696-12697, doi: 10.1021/ja037642h

Magariyama Y, Ichiba M, Nakata K, Baba K, Ohtani T, Kudo S, Goto T, 2005

"Difference in bacterial motion between forward and backward swimming caused by the wall effect"

Biophysical Journal 88, pp. 3648-3658, doi: 10.1529/biophysj.104.054049

Manoharan V N, Elsesser M T, Pine D J D, 2003

"Dense packing and symmetry in small clusters of microspheres"

Science 301, pp. 483-487, doi: 10.1126/science.1086189

Martel S, 2016

"Swimming microorganisms acting as nanorobots versus artificial nanorobotic agents: A perspective view from an historical retrospective on the future of medical nanorobotics in the largest known three-dimensional biomicrofluidic networks" Biomicrofluidics 10, p. 021301, doi: 10.1063/1.4945734 
Martel S, Mohammadi M, 2010

"Using a swarm of self-propelled natural microrobots in the form of flagellated bacteria to perform complex micro-assembly tasks"

Proceedings - IEEE International Conference on Robotics and Automation pp. 500-505, doi: 10.1109/ROBOT.2010.5509752

Martel S, Mohammadi M, Felfoul O, Lu Z, Pouponneau P, 2009

"Flagellated magnetotactic bacteria as controlled mri-trackable propulsion and steering systems for medical nanorobots operating in the human microvasculature" International Journal of Robotics Research 28, pp. 571-582, doi: 10.1177/0278364908100924

Mastrangeli M, Abbasi S, Varel C, van Hoof C, Celis J P, Böhringer K F, 2009

"Self-assembly from milli- to nanoscales: methods and applications" Journal of Micromechanics and Microengineering 19, pp. 1-37, doi: 10.1088/0960$1317 / 19 / 8 / 083001$

Maus S, Macmillan S, McLean S, Hamilton B, Thomson A, Nair M, Rollins C, December 2010 "The us/uk world magnetic model for 2010-2015" Technical report, British Geological Survey

Menciassi A, Quirini M, Dario P, 2007

"Microrobotics for future gastrointestinal endoscopy"

Minimally Invasive Therapy \& Allied Technologies 16, pp. 91-100

Messina R, Stankovic I, 2017

"Assembly of magnetic spheres in strong homogeneous magnetic field" Physica A: Statistical Mechanics and its Applications 466, pp. 10-20, doi: 10.1016/j.physa.2016.08.079

Messina R, Khalil L, Stankovic I, 2014

"Self-assembly of magnetic balls: From chains to tubes"

Physical Review E - Statistical, Nonlinear, and Soft Matter Physics 89, doi: 10.1103/PhysRevE.89.011202

Miyashita S, Nagy Z, Nelson B, Pfeifer R, 2009

"The influence of shape on parallel self-assembly"

Entropy 11, pp. 643-666, doi: 10.3390/e11040643

Miyashita S, Göldi M, Pfeifer R, 2011

"How reverse reactions influence the yield of self-assembly robots"

Int. J. Robot. Res. 30, pp. 627-641

Moisescu C, Bonneville S, Staniland S, Ardelean I, Benning L, 2011

"Iron uptake kinetics and magnetosome formation by magnetospirillum gryphiswaldense as a function of ph, temperature and dissolved iron availability"

Geomicrobiology Journal 28, pp. 590-600, doi: 10.1080/01490451.2011.594146

Murugesan Y, Pasini D, Rey A, 2015

"Self-assembly mechanisms in plant cell wall components"

Journal of Renewable Materials 3, pp. 56-72, doi: 10.7569/JRM.2014.634124

Nelson B, Kaliakatsos I, Abbott J, 2010

"Microrobots for minimally invasive medicine"

Annual Review of Biomedical Engineering 12, pp. 55-85, doi: 10.1146/annurev-bioeng-010510103409

Nelson H, Sargent D, Wieand H, Fleshman J, Anvari M, Stryker S, Beart Jr R, Hellinger M, Flanagan Jr R, Peters W, Ota D, 2004

"A comparison of laparoscopically assisted and open colectomy for colon cancer" New England Journal of Medicine 350, pp. 2050-2059+2114, doi: 10.1056/NEJMoa032651 
Norris D, Arlinghaus E, Meng L, Heiny R, Scriven L, 2004

"Opaline photonic crystals: How does self-assembly work?"

Advanced Materials 16, pp. 1393-1399+1385, doi: 10.1002/adma.200400455

Odar F, Hamilton W, 1964

"Forces on a sphere accelerating in a viscous fluid"

Journal of Fluid Mechanics 18, pp. 302-314, doi: 10.1017/S0022112064000210

Ojha R, Lemieux P, Dixon P, Liu A, Durian D, 2004

"Statistical mechanics of a gas-fluidized particle"

Nature 427, pp. 521-523

Pantaleone J, Messer J, 2011

"The added mass of a spherical projectile"

American Journal of Physics 79, pp. 1202-1210, doi: 10.1119/1.3644334

Park J K, Campos C D M, Neuzil P, Abelmann L, Guijt R M, Manz A, 2015

"Direct coupling of a free-flow isotachophoresis (ffitp) device with electrospray ionization mass spectrometry (esi-ms)"

Lab Chip 15, pp. 3495-3502, doi: 10.1039/C5LC00523J

Perrin J, 1909

"Brownian motion and molecular reality"

Annales de Chimie et de Physique 18, pp. 5-114

Philipse A, 1989

"Solid opaline packings of colloidal silica spheres"

J. Mater. Sci. Lett. 8, pp. 1371-1373, doi: 10.1007/BF00720190

Philipse A P, van Bruggen M P B, Pathmamanoharan C, 1994

"Magnetic silica dispersions: preparation and stability of surface-modified silica particles with a magnetic core"

Langmuir 10, pp. 92-99, doi: 10.1021/la00013a014

Pichel M P, Hageman T A G, Khalil I S M, Manz A, Abelmann L, 2018

"Magnetic response of magnetospirillum gryphiswaldense"

(under review)

Popp F, Armitage J, Schüler D, 2014

"Polarity of bacterial magnetotaxis is controlled by aerotaxis through a common sensory pathway"

Nature Communications 5, doi: 10.1038/ncomms6398

Pósfai M, Kasama T, Dunin-Borkowski R E, 2007

"Characterization of bacterial magnetic nanostructures using high-resolution transmission electron microscopy and off-axis electron holography"

In: D Schüler, editor, Magnetoreception and Magnetosomes in Bacteria, pp. 197-225, SpringerVerlag, Berlin, Berlin, Heidelberg

ISBN 978-3-540-37468-8, doi: 10.1007/7171_044

Press W H, Teukolsky S A, Vetterling W T, Flannery B P, 1992

Numerical Recipes in C (2nd Ed.): The Art of Scientific Computing,

Cambridge University Press

ISBN 0-521-43108-5

Reufer M, Besseling R, Schwarz-Linek J, Martinez V, Morozov A, Arlt J, Trubitsyn D b, Ward F, Poon W, 2014

"Switching of swimming modes in magnetospirillium gryphiswaldense"

Biophysical Journal 106, pp. 37-46, doi: 10.1016/j.bpj.2013.10.038 
Richardson L, 1922

Weather prediction by numerical process,

Cambridge University Press

Richardson L F, 1926

"Atmospheric diffusion shown on a distance-neighbour graph"

Proceedings of the Royal Society of London. Series A, Containing Papers of a Mathematical and Physical Character 110, pp. 709-737, doi: 10.1098/rspa.1926.0043

Roland J C, Reis D, Vian B, 1992

"Liquid crystal order and turbulence in the planar twist of the growing plant cell walls" Tissue and Cell 24, pp. 335-345, doi: 10.1016/0040-8166(92)90050-H

Rossi L, Sacanna S, Irvine W T M, Chaikin P M, Pine D J, Philipse A P, 2011

"Cubic crystals from cubic colloids"

Soft Matter 7, pp. 4139-4142, doi: 10.1039/C0SM01246G

Rothemund P W K, 2006

"Folding DNA to create nanoscale shapes and patterns"

Nature 440, pp. 297-302, doi: doi:10.1038/nature04586

Rycenga M, McLellan J M, Xia Y, 2008

"Controlling the assembly of silver nanocubes through selective functionalization of their faces”

Advanced Materials 20, pp. 2416-2420, doi: 10.1002/adma.200800360

Schleifer K, Schuler D, Spring S, Weizenegger M, Amann R, Ludwig W, Kohler M, 1991 "The genus magnetospirillum gen. nov. description of magnetospirillum gryphiswaldense sp. nov. and transfer of aquaspirillum magnetotacticum to magnetospirillum magnetotacticum comb. nov."

Systematic and Applied Microbiology 14, pp. 379-385

Schüler D, Baeuerlein E, 1998

"Dynamics of iron uptake and fe3o4 biomineralization during aerobic and microaerobic growth of magnetospirillum gryphiswaldense"

Journal of Bacteriology 180, pp. 159-162

Schüler D, Uhl R, Bäuerlein E, 1995

"A simple light scattering method to assay magnetism in magnetospirillum gryphiswaldense" FEMS Microbiology Letters 132, pp. 139-145, doi: 10.1016/0378-1097(95)00300-T

Shetye S, Eskinazi I, Arnold D, 2008

"Self-assembly of millimeter-scale components using integrated micromagnets"

IEEE Transactions on Magnetics 44, pp. 4293-4296, doi: 10.1109/TMAG.2008.2001344

Shetye S, Eskinazi I, Arnold D, 2010

"Magnetic self-assembly of millimeter-scale components with angular orientation"

Journal of Microelectromechanical Systems 19, pp. 599-609, doi: 10.1109/JMEMS.2010.2042681

Smid P, Shcherbakov V, Petersen N, 2015

"Microscopic observation of magnetic bacteria in the magnetic field of a rotating permanent magnet"

Review of Scientific Instruments 86, doi: 10.1063/ 1.4929331

Smoluchowski M V, 1916

"Drei vortrage uber diffusion, Brownsche bewegung und koagulation von kolloidteilchen"

Zeitschrift fur Physik 17, pp. 557-585 
Song T, Zhao L, Wu L F, 2014

"A method for quantitative determination of the number of magnetosomes in magnetotactic bacteria by a spectrophotometer"

IEEE Transactions on Magnetics 50, doi: 10.1109/TMAG.2014.2323953

Stambaugh J, Lathrop D, Ott E, Losert W, 2003

"Pattern formation in a monolayer of magnetic spheres"

Physical Review E - Statistical, Nonlinear, and Soft Matter Physics 68, pp. 026207/1-026207/5

Taherkhani S, Mohammadi M, Daoud J, Martel S, Tabrizian M, 2014

"Covalent binding of nanoliposomes to the surface of magnetotactic bacteria for the synthesis of self-propelled therapeutic agents"

ACS Nano 8, pp. 5049-5060, doi: 10.1021/nn5011304

Tanaka H, Kido M, Yahashi K, Oomura M, Katsumata R, Kito M, Fukuzumi Y, Sato M, Nagata Y, Matsuoka Y, Iwata Y, Aochi H, Nitayama A, 2007

"Bit cost scalable technology with and plug process for ultra high density flash memory" Digest of Technical Papers - Symposium on VLSI Technology pp. 14-15, doi: 10.1109/VLSIT.2007.4339708

Tricard S, Stan C A, Shakhnovich E I, Whitesides G M, 2013

"A macroscopic device described by a boltzmann-like distribution"

Soft Matter 9, pp. 4480-4488

Tricard S, Shepherd R F, Stan C A, Snyder P W, Cademartiri R, Zhu D, Aranson I S, Shakhnovich E I, Whitesides G M, 2015

"Mechanical model of globular transition in polymers"

ChemPlusChem 80, pp. 37-41

Uebe R, Schüler D, 2016

"Magnetosome biogenesis in magnetotactic bacteria"

Nature Reviews Microbiology 14, pp. 621-637, doi: 10.1038/nrmicro.2016.99

Wang S, Wolynes P, 2011

"Communication: Effective temperature and glassy dynamics of active matter"

Journal of Chemical Physics 135, doi: 10.1063/1.3624753

Whitelam S, Jack R, 2015

"The statistical mechanics of dynamic pathways to self-assembly"

Annual Review of Physical Chemistry 66, pp. 143-163, doi: 10.1146/annurev-physchem040214-121215

Whitesides G M, Boncheva M, 2002

"Beyond molecules: Self-assembly of mesoscopic and macroscopic components"

P. Natl. Acad. Sci. USA 99, pp. 4769-4774

Whitesides G M, Grzybowski B, 2002

"Self-assembly at all scales"

Science 295, pp. 2418-2421, doi: 10.1126/science.1070821

Witt A, Fabian K, Bleil U, 2005

"Three-dimensional micromagnetic calculations for naturally shaped magnetite: Octahedra and magnetosomes"

Earth and Planetary Science Letters 233, pp. 311-324, doi: 10.1016/j.epsl.2005.01.043

Woldering L A, Been A J, Alink L, Abelmann L, 2016

"Using magnetic levitation for 2D and 3D self-assembly of cubic silicon macroparticles"

Physica status solidi RRL 10, pp. 176-184, doi: 10.1002/pssr.201510298 
Wolfe R S, Thauer R K, Pfenning N, 1987

"A 'capillary racetrack' method for isolation of magnetotactic bacteria" FEMS Microbiology Ecology 45, pp. 31-35

Yang C, Takeyama H, Matsunaga T, 2001a "Iron feeding optimization and plasmid stability in production of recombinant bacterial magnetic particles by magnetospirillum magneticum amb-1 in fed-batch culture" Journal of Bioscience and Bioengineering 91, pp. 213-216, doi: 10.1016/S1389-1723(01)80068-2

Yang C, Chen C, Ma Q, Wu L, Song T, 2012

"Dynamic model and motion mechanism of magnetotactic bacteria with two lateral flagellar bundles"

Journal of Bionic Engineering 9, pp. 200-210, doi: 10.1016/S1672-6529(11)60108-X

Yang C D, Takeyama H, Tanaka T, Matsunaga T, 2001b

"Effects of growth medium composition, iron sources and atmospheric oxygen concentrations on production of luciferase-bacterial magnetic particle complex by a recombinant magnetospirillum magneticum amb-1"

Enzyme and Microbial Technology 29, pp. 13-19, doi: 10.1016/S0141-0229(01)00343-X

Yang Y, Lopez G P, Yellen B B, 2013

"Tunable assembly of colloidal crystal alloys using magnetic nanoparticle fluids"

ACS Nano 7, pp. 2705-2716

Yener A, Klapp S, 2016

"Self-assembly of three-dimensional ensembles of magnetic particles with laterally shifted dipoles"

Soft Matter 12, pp. 2066-2075, doi: 10.1039/c5sm02648b

Zahn C, Keller S, Toro-Nahuelpan M, Dorscht P, Gross W, Laumann M, Gekle S, Zimmermann W, Schüler D, Kress H, 2017

"Measurement of the magnetic moment of single magnetospirillum gryphiswaldense cells by magnetic tweezers"

Scientific Reports 7, doi: 10.1038/s41598-017-03756-Z

Zhang Z, Keys A, Chen T, Glotzer S, 2005

"Self-assembly of patchy particles into diamond structures through molecular mimicry"

Langmuir 21, pp. 11547-11551, doi: 10.1021/la0513611

Zhao L, Wu D, Wu L F, Song T, 2007

"A simple and accurate method for quantification of magnetosomes in magnetotactic bacteria by common spectrophotometer"

Journal of Biochemical and Biophysical Methods 70, pp. 377-383, doi:

10.1016/j.jbbm.2006.08.010

Zheng W, Buhlmann P, Jacobs H, 2004

"Sequential shape-and-solder-directed self-assembly of functional microsystems"

Proceedings of the National Academy of Sciences of the United States of America 101, pp. 1281412817, doi: 10.1073/pnas.0404437101 


\section{Abstract}

Observation of the motion of particles in fluids give valuable information about the particles, the environment and the interaction between them. Two distinct particle-fluid systems were studied in this framework.

The first system considers centimetre-sized magnetic particles suspended in an upward water flow to create neutral buoyancy as well as a source of turbulence. This macroscopic reactor acts as an analogue simulator for microscopic self-assembly processes. From observation of the trajectories of single and twoparticle systems we found that in terms of velocity, diffusion and particle interaction the laws of thermodynamics describe the macroscopic system with surprising accuracy. We have shown that we can control the amount of disturbing energy by changing the asymmetry of the water inflow, but that this method affects the particle behaviour differently in separate spatial dimensions. We found that the method used to generate disturbing energy is not that critical; also when the particles are mechanically shaken on a table in $2 \mathrm{D}$, rather than in a turbulent flow in 3D, the velocity and diffusion still obey the laws of thermodynamics.

The macroscopic reactor was used to study self-assembly of 3D-printed objects with embedded magnets. A system of four spheres was analysed by both humans and neural networks. Although yielding very similar results, they significantly deviate from theoretical predictions, likely due to underestimation of the disturbing energy. When using objects with anisotropic shape, we found that the geometry and aspect ratio highly define the nature of resulting structures. The particle shape for instance controls the dimensionality (1D, 2D, 3D) and flexibility (straight versus flexible angles) of the resulting assemblies.

The second system involves the study of the motion of magnetotactic bacteria (MTB) under influence of varying magnetic fields. From microscopy observations of the trajectories of individual MTB we found that their response to magnetic fields accurately follows a simple model based on the ratio between magnetic and drag torque. We characterised the properties of MTB and interaction with the environment. An optical density based method was developed to monitor the properties of entire colonies of MTB with high temporal resolution. We were able to monitor four distinct parameters corresponding to growth and magnetic growth of MTB and found that these types of growth are decoupled.

Although magnetic objects studied in this thesis are seemingly very distinct, 
with various sizes and shapes, their analysis has strong similarity. The most important aspects for fluid-particle interaction are the interplay between magnetic torque and the drag force as well as the interplay between magnetic potential energy and (equivalent) thermal energy. The parameters underpinning the models based on these concepts can be determined through observation of the motion of the particles. 


\section{Samenvatting}

Van de beweging van magnetische deeltjes in vloeistoffen kunnen we veel leren over de deeltjes zelf, de omgeving en hun interactie. In deze thesis zijn twee systemen bestudeerd.

Het eerste systeem probeert meer inzicht te geven over hoe microscopische deeltjes interactie met elkaar ondervinden. Onder bepaalde omstandigheden vormen deze deeltjes spontaan structuren, een proces dat we zelf-assemblage noemen. Zulke processen zijn onder normale omstandigheden moeilijk direct te observeren door de kleine afmetingen en de zeer korte tijdschaal waarop de bewegingen plaatsvinden. Aan de andere kant zouden we deze processen kunnen simuleren met computers, maar deze berekeningen zijn zeer tijdsintensief en schalen ongunstig met het aantal deeltjes. Als alternatief hebben we een reactor gebouwd die de zelf-assemblage processen (analoog) op centimeterschaal simuleert om alles beter zichtbaar te maken. Deze drie-dimensionale macroscopische reactor balanceert magnetische deeltjes in een opwaartse, turbulente waterstroom die zowel hun zwaartekracht neutraliseert als een bron is van verstorende energie. De verstoring is analoog voor de willekeurige beweging die deeltjes op kleine schaal ondervinden als gevolg van temperatuur. Door met camera's de beweging van de deeltjes te volgen hebben we geconcludeerd dat ze opmerkelijke overeenkomsten hebben met het theoretische gedrag van microscopische deeltjes. De hoeveelheid verstorende energie kan geregeld worden door de asymmetrie van de toevoer van de waterstroom te variëren. Dit heeft een verschillende invloed op de horizontale en verticale componenten van de beweging van de deeltjes. De manier waarop we energie toevoeren blijkt niet zo belangrijk; ook wanneer deeltjes op een twee-dimensionaal vlak worden geschud is de overeenkomst met thermodynamica opvallend.

Zelf-assemblage processen waren onderzocht door de interactie van grotere aantallen 3D geprinte deeltjes met een magnetische kern te bestuderen. Van vier balletjes was onderzocht hoe vaak deze een lijn- en ring-vorm aannamen. Zowel handmatige als geautomatiseerd (neurale netwerken) analyse tonen aan dat de frequentie overeenkomt met wat de statistische mechanica voorspelt. De lengteverhouding en geometrie van de deeltjes hebben een overweldigende invloed op de resulterende structuren. Deze bepalen zowel de dimensionaliteit (1D, 2D, 3D) als de karakteristieke verbindingen (flexibele versus rechte hoeken) tussen de deeltjes. 
Als tweede systeem zijn magnetotactische bacteriën (MTB) bestudeerd. Deze zwemmende bacteriën hebben een interne ketting van magnetische deeltjes waardoor hun zwemrichting, net zoals een kompasnaald, met behulp van magnetische velden kan worden beïnvloed. In de natuur gebruiken ze dit om zich te oriënteren in de richting van het aardmagnetisch veld. Op deze manier veranderen ze een willekeurige drie-dimensionale zoektocht naar hun ideale habitat naar een efficiëntere één-dimensionale zoektocht. Door velden significant sterker dan het aardmagnetisch veld te genereren kunnen we deze bacteriën gebruiken als micro-robots, mogelijkerwijs voor toepassing als het lokaal afleveren van medicijnen in het menselijke lichaam. We hebben het effect bestudeerd van magnetische velden op MTB door hun beweging onder de microscoop te bestuderen. Uit zwemtrajecten is bepaald dat de invloed van velden op MTB nauwkeurig beschreven wordt volgens een simpel model waarin de verhouding tussen het magnetisch koppel en rotationele wrijving centraal staat. Naast de karakterisatie van individuele MTB is de groei van complete kolonies bepaald door de transparantie van het de cultuur over tijd te meten. Op deze manier kunnen vier parameters gemeten worden die corresponderen met groei van MTB en de ontwikkeling van hun magnetisch moment. We hebben bepaald dat deze groeitypen onafhankelijk zijn.

Alhoewel deze projecten op het eerste gezicht compleet verschillend zijn, hebben ze belangrijke overeenkomsten. In beiden speelt de interactie van het magnetisch koppel en de rotationele wrijving een grote rol, evenals de verhouding tussen magnetische potentiële- en thermische energie. Door het observeren van de bewegingen van de deeltjes kunnen belangrijke parameters van de deeltjes en de omgeving worden bepaald. 


\section{Zusammenfassung}

Beobachtung der Bewegung von Partikeln in Flüssigkeiten bringt wertvolle Informationen über die Partikel, die Umgebung als auch die Interaktion von beidem. Zwei verschiedene Partikel-Flüssigkeitssysteme wurden in dieser Studie näher untersucht.

Das erste System setzte sich zusammen aus zentimeter-großen magnetischen Partikeln, ausgebracht in einem aufwärtsgerichteten Wasserstrom, welcher einen neutralen statischen Auftrieb erzeugte als auch den Ursprung von Turbulenzen darstellt. Dieser makroskopische Reaktor wurde betrieben als analoge Simulation für mikroskopische Selbstassemblierungsprozesse. Durch das Beobachten der Trajektorien von Ein- sowie Zwei-Partikelsystemen wurde festgestellt, dass die Gesetze der Thermodynamik überraschend genau das System charakterisieren, vor allem in Bezug auf Geschwindigkeit, Diffusion und Partikel-Interaktion. Wir konnten zeigen, dass wir die Stärke der Störenergie kontrollieren können durch Änderung der Asymmetrie des Wassereinlasses, aber auch das diese Methode die Partikel unterschiedlich beeinflusst, je Lage im dreidimensionalen Raum. Es konnte nachgewiesen werden, dass die Methode zur Erzeugung der Störenergie kein kritischer Einflussfaktor ist, da auch beim mechanischen Schütteln von Partikeln auf einem Tisch in 2D, im Gegensatz zu einer turbulenten Flussrate in 3D, Geschwindigkeit und Diffusion weiterhin den Gesetzen der Thermodynamik unterliegen.

Der makroskopische Reaktor wurde zur Untersuchung von Selbstassemblierungsprozessen von 3D-gedruckten Objekten mit eingeschlossenen Magneten verwendet. Ein System aus vier Kugeln wurde sowohl durch Probanden als auch durch Neurale Computernetzwerk analysiert. Trotz der sehr ähnlichen Ergebnisse konnte ein signifikanter Unterschied zu den theoretischen Vorhersagen festgestellt werden, welcher höchstwahrscheinlich in der Unterschätzung der Störenergie begründet war. Bei der Benutzung von Objekten mit anisotropen Formen konnten wir zeigen, dass die Geometrie sowie das Seitenverhältnis starken Einfluss nehmen auf die entstehenden Strukturen. Die Form der Partikel hat beispielsweise entscheidenden Einfluss auf die Dimensionalität (1D, 2D, 3D) und Flexibilität (Grade vs. Flexible Winkel) der entstehenden Verbindungen.

Das zweite System umfasste die Analyse der Bewegung von magnetotaktischen Bakterien (MTB) unter Einfluss von wechselnden Magnetfeldern. Durch 
mikroskopische Beobachtung der Bewegungsbahnen von einzelnen MTB konnten wir nachweisen, dass deren Bewegungsantwort auf magnetische Felder exakt einem einfachen Modell folgen, basierend auf dem Verhältnis zwischen magnetischem Drehmoment und Dreh-Strömungswiderstandes. Hierzu wurden die Eigenschaften der MTB und deren Interaktion mit der Umgebung charakterisiert. Eine Methode, basierend auf optischer Dichte-Messung, wurde entwickelt um Eigenschaften von ganzen Kolonien von MTBs mit hoher zeitlicher Auflösung zu untersuchen. Es war uns möglich vier verschiedene Parameter bezüglich Wachstum und Wachstum der magnetischen Partikel zu überwachen um festzustellen, dass diese Typen des Wachstums sich als entkoppelt darstellen.

Obwohl die in dieser Doktorarbeit verwendeten magnetischen Objekte stark unterschiedlich in Bezug auf Größe und Form waren zeigte deren Auswertung hohe Ähnlichkeiten. Der wichtigste Aspekt der Partikel-Flüssigkeitsinteraktion stellt das Zusammenspiel von magnetischem Drehmoment und des Dreh-Strömungswiderstandes dar, als auch das Zusammenspiel der potenziellen magnetischen Energie und der (äquivalenten) thermalen Energie. Diese Parameter der Modelle konnten durch Beobachtung der Bewegung der Partikel untermauern werden, auf welchen die Konzepte des magnetischen Moments und des Strömungswiderstandes basieren. 


\section{초록}

유체 내 입자의 움직임을 관찰하는 것은 입자와 환경은 물론 그들 간의 상호 작 용에 대한 중요한 정보를 제공한다. 본 논문에서는 두 가지의 상이한 입자-유체 시스템을 연구하였다.

첫 번째, 상 방향 유체 흐름으로 기인한 난류에 의해 부유되는 센티미터 크 기의 자성 입자에 관한 연구이다. 미세 입자의 자기 조립/조합 과정을 분석하기 위한 장치로서 거대 입자 반응기가 사용되었다. 단일 및 이중 입자 시스템의 궤 적을 관찰한 결과, 속도, 확산, 입자의 상호 작용의 세 가지 측면에서 거대 입자 반응기의 정확도는 열역학 법칙과 놀라울 정도로 정확하게 일치한다는 점이 입 증되었다. 본 연구에서는 유입되는 물의 비대칭 성을 변화시킴으로써 방해하는 에너지의 양을 제어 할 수 있었고, 이 방법은 별도의 공간 차원에서 입자 행동에 다르게 영향을 미친다는 것을 보여주었다. 또한, 본 연구를 통해 방해하는 에너 지를 생성하는 데 사용되는 방법은 그렇게 중요하지 않다는 점이 발견되었으며, 난기류 상의 $3 \mathrm{D}$ 입자이든지 테이블 위에서 기계적으로 흔들리는 $2 \mathrm{D}$ 입자이든 지도속도와 확산은 여전히 열역학의 법칙을 따른다는 점이 발견되었다.

거대 입자 반응기는 자석이 내장되어 $3 \mathrm{D}$ 로 인쇄된 객체의 자기 조립을 연구 하는 데에도 사용되었다. 4 개의 구 모양으로 만들어진 모델을 이용하여 인간과 컴퓨터의 신경 회로망을 분석을 하였다. 위 두 수치는 비슷한 산출 결과를 보였 으나, 이론적 예측 값에는 빗겨나가는 경향을 보였으며, 이것은 불안한 에너지로 부터 기인한 것으로 추론된다. 그 예로써, 입자 모양은 조립된 결과물의 공간의 크기 (1D, 2D, 3D) 및 유연성 (직선, 유연 각도)을 결정한다.

두 번째는 다양한 자기장의 영향을 받는 자성 미생물 (magnetotactic bacteria, MTB) 의 운동에 관한 연구이다. 개별 자성 미생물들의 궤적을 현미경으로 관찰한 결과, 각 세균의 자기장 반응이 자기 및 회전 항력 사이의 비율에 기초한 기본 모델을 정확하게 따르는 것으로 나타났으며, 자성 미생물의 특징 및 환경 과의 상호 작용을 규정할 수 있었다. 고 해상도 관찰을 통해 자성 미생물의 전 체적인 특성을 모니터링하기 위하여 광학 밀도 측정 방법이 사용되었으며, 이를 통해 주자성 세균의 성장과 자성 성장에 해당하는 네 가지 매개 변수를 모니터링 할 수 있었고 이러한 두 유형의 성장은 각각 분리되어 있음을 발견하였다.

본 논문에서 연구 된 자성 물체는 다양한 크기와 모양으로 인해 겉으로는 매 우 상이해 보이지만, 분석 결과 유사성이 매우 높은 것으로 입증되었다. 유체입자 상호 작용의 경우 자기포텐셜 에너지와 열 에너지 사이의 상호 작용뿐만 
아니라 자기토오크와 드래그 포스 사이의 상호 작용이 가장 중요하며, 이러한 개념에 기반을 둔 모델을 뒷받침하는 매개 변수는 입자 운동의 관찰을 통해 규 정될 수 있다. 


\section{Acknowledgments}

This thesis would never have been here without the direct and indirect support of many others. I tried to include all people that deserve my sincere thanks for this. Unfortunately I am human and therefore inherently flawed, and could accidentally have left you out (sorry); in that case please notify me and I'm happy to write you a personal message in this copy.

Leon, you once told me the most important decision you make during a $\mathrm{PhD}$ is right at the start; choosing your supervisor. I don't think I could have made a better choice; you have been an amazing boss and supervisor for the past four years. Thank you for the never-wavering support you've offered, for the thorough scientific (and not-so-scientific) discussions, for the many things you've taught me and for always pushing me to go that little bit further.

Marc, we've been working together since the start and it was always a pleasure to work side by side. But more importantly, you have been a good friend and influenced me for the better to a greater extent than you probably think. Thank you for your effort on MTB, the philosophical conversations, amazing road trips, occasional beer at Murphy's, your role as paranymph and more. It's an honour to cross the finish line together!

Galina, we've shared many adventures, moments and trips together, sometimes taking us to the other side of the world. Благодарю за поддержку!

To all of my collegues at KIST: thank you for the discussions, scientific help, lunch- and coffee breaks, the occasional movie evening and general laughs, but most of all for making it a great working place; Andreas, André-René, Bohyun, Camilla, Christian, Eric, Felix, Jingeum (한국 수업에 감사합니다), Jonathan, Jukyung, Jang Mi, Levent, Neha, Nuriye, Pavil, Per, Ruth, Rosanne, Sabrina, Seungjae, Susanne, Thais, Vanessa (shu-shu!) and Ferdia, Xiangping and all other people at KIST.

Michael, Dhananjay and Massimo, Emmanual and Franziska, thank you for the pleasant and fruitful collaboration. Islam and Elwi, thank you for your collaboration on the MTB and for your hospitality in Egypt. Henk, thank you for sharing your wisdom on microscopy. Léon and Remco, thank you for your valuable contribution to the self-assembly reactor. Gijs, Dave, Kodai, Laurens and Marcel, thank you for the support from Twente. 
Thank you very much, Wesley, for your hospitality and introducing me to machine learning. Thank you Jasper, Mijung and all the other amazing people at Ghent University Global Campus to make my stay unforgettable.

My gratitude goes to the people generally not in the spotlight, but who provide immense support and keep the institute running. Thank you Carsten, Holger, Jean-Philippe, Rüdiger, Sabine and Thomas. A special thanks goes to Matthias, for supporting the early research with MTB, for translating part of this thesis, for your enthusiasm and for never being too tired to explain something a third time.

This thesis would not be here in this format without the help of the many students during my time at KIST. Not only did you make valuable contributions; working with you was interesting, versatile and fun. Thank you Abdelrahman, Alveena, Debasish, Deepti, Donghoon, Gayoung, Hans, Heba, Helen, Jordi, Karim, Negi, Niko, Maram, Noura, Ola, Osama, Sangin, Tim, William and Yannick.

Thank you, members of the committee, for making this defence possible; Leon Abelmann, Miko Elwenspoek, Joost Kok, Tobias Kraus, Gijs Krijnen, Andreas Manz, Matthias Nienhaus and Cees Slump.

Een warm dankjewel is gereserveerd voor mijn familie, die me altijd bijgestaan hebben in mijn keuze om naar het buitenland te verhuizen en die me ook regelmatig hebben opgezocht. Dankjewel mam, pap, Jolijn, Kaspar (ook voor je rol als paranymph), Remi en Yara. Bedankt ome Frank, tante Riky, ome Theo, tante Wilma en alle andere familieleden.

Mijn vrienden terug in Nederland hebben me, ondanks de grote afstand, nooit in de steek gelaten. Bedankt Cynthia, Elisa, Esmay, Frank, Ivo, Jasper, Jeroen, Lisette, Nick, René, en Vera, voor de dagelijkse zin en onzin via Whatsapp en de gezellige avondjes in Nederland. Frank en Nick, bedankt voor de (soms letterlijke) hoogtepunten in het land van de rijzende zon.

I have had the honour of sharing an ever-cosy house with amazing people. Thank you Aurore, Eva, Kristina, Omer, Paulina, Sviat and Tessy for the movie evenings, analogue and digital games, occasional beer and everything else! Thank you Elisa, Ilya, Irina, Jérôme, Maria, Markus, Michael, Riccardo, Samir, Sasha and all others I met in Saarbrücken for the adventures and experiences. 


\section{Publications}

\section{Journal articles}

Hageman T A G, Löthman P A, Dirnberger M, Elwenspoek M, Manz A, Abelmann L, 2018 "Macroscopic equivalence for microscopic motion in a turbulence driven three-dimensional self-assembly reactor" Journal of Applied Physics 123, p. 024901, doi: 10.1063/1.5007029

Hageman T A G, Löthman P A, Manz A, Abelmann L, 2018a

"Macroscopic cluster formation of magnetic particles in a three-dimensional self-assembly reactor"

(in preparation)

Hageman T A G, Pichel M P, Hendrix J, Keizer H, Manz A, Abelmann L, 2018b

"Real-time monitoring of growth and magnetic properties of magnetotactic bacteria via optical density"

(in preparation)

Ipparthi D, Hageman T A G, Cambier N, Sitti M, Dorigo M, Abelmann L, Mastrangeli M, 2018 "Kinetics of 2d constrained orbitally-shaken particles" (under review) arXiv:1710.09249

Löthman P A, Hageman T A G, Dirnberger M, Manz A, Abelmann L, 2018 "Characterisation of the disturbing energy in a macroscopic self-assembly reactor" (in preparation)

Lötters J C, Lammerink T S J, Haneveld J, Hageman T A G, Wiegerink R J, 2012

"Fully integrated micro coriolis mass flow sensor operating at atmospheric pressure [ein voll integrierter mikro-coriolis-durchflusssensor für atmosphärendruck]"

Technisches Messen 79, pp. 4-9, doi: 10.1524/teme.2012.0171

Pichel M P, Hageman T A G, Khalil I S M, Manz A, Abelmann L, 2018

"Magnetic response of magnetospirillum gryphiswaldense"

(under review) arXiv:1710.00405

Terriac E, Coceano G, Mavajian Z, Hageman T A G, Christ A F, Testa I, Lautenschläger F, Gad A K B, 2017

"Vimentin levels and serine 71 phosphorylation in the control of cell-matrix adhesions, migration speed, and shape of transformed human fibroblasts"

Cells 6, doi: 10.3390/cells6010002 


\section{Conference contributions}

Abelmann L, Löthman P, Hageman T, Pichel M, Manz A, 2016a

"Magnetically assisted three-dimensional self-assembly"

In: Joint MMM-Intermag Conference,

Abelmann L, Pichel M, Hageman T, Manz A, $2016 b$

"Response of magneto-tactic bacteria to a rotating magnetic field"

In: Joint MMM-Intermag Conference,

Hageman T, Pichel M, Altmeyer M, Manz A, Abelmann L, 2015a

"Planar manipulation of magneto-tactic bacteria using unidirectional magnetic fields" In: MicroTAS 2015 - 19th International Conference on Miniaturized Systems for Chemistry and Life Sciences, pp. 716-718,

Hageman T A G, Pichel M P, Altmeyer M O, Abelmann L, Manz A, 2014

"An integrated magnet array for trapping and manipulation of magnetotactic bacteria in microfluidics"

In: Micromechanics and microsystems Europe workshop,

Hageman T A G, Löthman P A, Janson T G, Manz A, Abelmann L, 2015 b

"Characterization of a macroscopic self-assembly reactor"

In: Micromechanics and microsystems Europe workshop,

Hageman T A G, Löthman P A, Woldering L, Bienia N, Manz A, Abelmann L, $2015 c$

"Disturbing energy in a macroscopic self-assembly reactor"

In: FNANO15 - Conference on foundations of nanoscience: self-assembled structures and

devices,

Hageman T A G, Löthman P A, Manz A, Abelmann L, 2016

"Characterization of a macroscopic self-assembly reactor"

In: Micromechanics and microsystems Europe workshop,

Hageman T A G, Dirnberger M, Elwenspoek M, Krijnen G, Löthman P A, Manz A, Pichel M P, Abelmann L, 2017

“Turbulence-driven macroscopic magnetic self-assembly with adjustable level of agitation" In: Intermag2017,

Hageman T A G, Löthman P A, Manz A, Abelmann L, 2017a

"Analysis of dipolar chain and ring formation in a macroscopic self assembly reactor"

In: Micromechanics and microsystems Europe workshop,

Hassan H, Pichel M, Hageman T, Abelmann L, Khalil I S M, 2016

"Influence of the magnetic field on the two-dimensional control of magnetospirillum gryphiswaldense strain msr-1"

In: Proceedings of the IEEE International Conference on Intelligent Robots and Systems (IROS), Daejeon, Korea, pp. 5119-5124,

Khalil I S M, Tabak A F, Hageman T, Ewis M, Pichel M, Mitwally M E, El-Din N S, Abelmann L, Sitti M, 2017

"Near-surface effects on the controlled motion of magnetotactic bacteria" pp. 5976-5982, doi: 10.1109/ICRA.2017.7989705

Löthman P, Woldering L, Hageman T, Abelmann L, Manz A, 2014

"Towards biomimetic microfabrication via three-dimensional macroscopic magnetic selfassembly"

In: Micromechanics and microsystems Europe workshop, 
Löthman P A, Hageman T A G, Woldering L, Bienia N J, Elwenspoek M, Manz A, Abelmann L, 2015 "Macroscopic self-assembly via turbulent flow and magnetic interaction" In: FNANO15 - Conference on foundations of nanoscience: self-assembled structures and devices,

Löthman P A, Hageman T A G, Manz A, Abelmann L, 2016

"Turbulence as the disturbing force in macroscopic self-assembly" In: Micromechanics and microsystems Europe workshop,

Pichel M, Hageman T, Altmeyer M, Abelmann L, Manz A, $2014 a$ "Magnetic manipulation of bacteria in microfluidics" In: MicroTAS 2014, 18th International Conference on Miniaturized Systems for Chemistry and Life Sciences, pp. 721-723,

Pichel M, Hageman T A G, Manz A, Abelmann L, 2016

"Towards measuring motile magnetic fractions of magnetotactic bacterial cultures" In: Micromechanics and microsystems Europe workshop,

Pichel M P, Hageman T A G, Altmeyer M O, Abelmann L, Manz A, $2014 b$

"Dynamics and magnetic control of magnetotactic bacteria u-turn trajectories in microfluidics" In: Micromechanics and microsystems Europe workshop,

Pichel M P, Hageman T A G, Altmeyer M O, Abelmann L, 2015 "U-turn trajectories of magnetotactic bacteria in microfluidics" In: Micromechanics and microsystems Europe workshop,

van Alphen M J A, Hageman T A G, Smeele L E, Balm A J M, van der Heijden F, 2013 "Predictive model for functional consequences of oral cavity tumour resections" In: Proceedings of the 27th International Congress and Exhibition on Computer Assisted Radiology and Surgery, CARS 2013, pp. 156-157, Springer, doi: 10.1007/s11548-013-0864-0 



\section{Biography}

Tijmen Hageman was born on the $25^{\text {th }}$ of May 1989 in the village of Didam, the Netherlands. He enjoyed an education at the Liemers College, where he discovered a passion for science and engineering. In 2007 he moved to Enschede to study Electrical Engineering at the University of Twente. He received his BSc in 2011 and his MSc (cum laude) in 2013 after finishing his thesis on tracking the human tongue in 3D using a multi-camera system. In this period he also completed an internship at radar company Thales in Hengelo, and attended a study tour to Asia, which awakened his passion for traveling.

In 2014 he started as a PhD candidate at KIST Europe under supervision of Leon Abelmann, of which the result is this very thesis. During this period he supervised many students and taught several courses at the Saarland University. He also did an internship at the Ghent University Global Campus in Incheon, South-Korea to gain knowledge about machine learning.

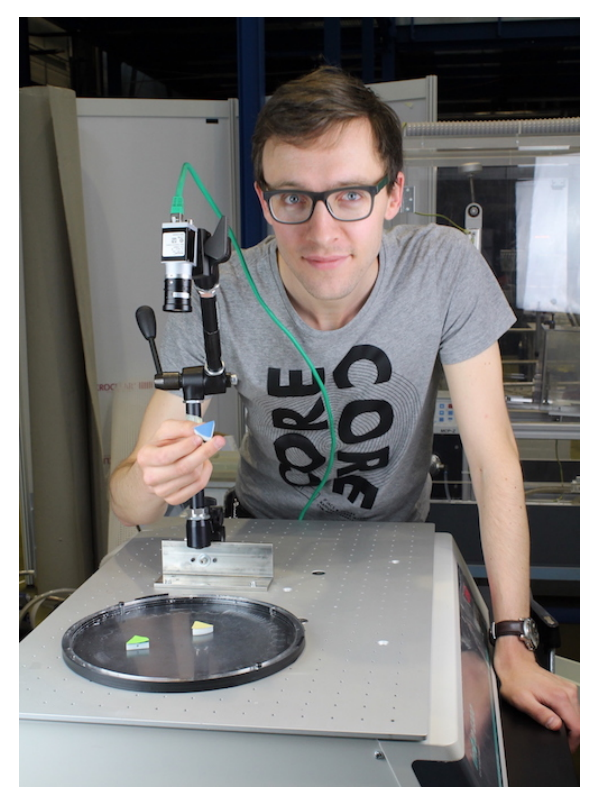





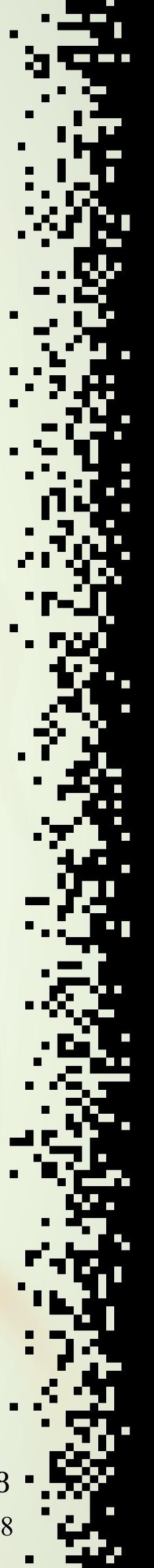

